\title{
Vision based real-time navigation with Unknown and Uncooperative Space Target
}

by

Setareh Yazdkhasti

A thesis submitted to the

Faculty of Graduate and Postdoctoral Affairs

in partial fulfillment of the requirements for the degree of

Doctor of Philosophy in Aerospace Engineering

Ottawa-Carleton Institute for Mechanical and Aerospace Engineering

Department of Mechanical and Aerospace Engineering

Carleton University

Ottawa, Ontario

May, 2021

(C)Copyright

Setareh Yazdkhasti, 2021 


\section{Abstract}

Hundreds of satellites are launched every year, and the failure rate during their operational time frame, combined with the four to five percent failure rate of launch vehicles means that approximately one of every seven satellites will fail before reaching their planned working life. This generates an enormous amount of space debris, which can be a danger to working space platforms such as the International Space Station (ISS), shuttles, spacecrafts, and other vehicles. If debris collides with a vehicle with human passengers, it could cause significant damage and threaten the life and safety of those aboard, plus the collisions themselves add more junk, thereby creating more risk. Thus, active space debris removal operations are essential. The removal of space debris requires pose (position and orientation) information and motion estimation of targets; thus, real-time detection and tracking of uncooperative targets are critical. In order to estimate a target's pose and motion, it must track over the entire frame. In addition, due to the harsh space environment, different types of sensors are required, which means practical sensor fusion algorithms are essential. However, as there is little prior information about a target's structure and motion, detection and tracking, pose and motion estimation and multi-sensor fusion tasks are very challenging.

This thesis develops new approaches for target tracking, pose and motion estimation and multi-sensor fusion. For the target-tracking step, a new adaptation scheme 
of the Unscented Kalman Filter (UKF) is proposed to manage difficulties associated with real-time tracking of an uncooperative space target. The proposed method employs fuzzy logic adaptation mechanisms incorporated in UKF to predict the location of a feature point in consecutive video frames, which allows for robust tracking. Furthermore, it can also help overcome some of the difficulties involved in space target tracking, such as the disappearance of a feature point, fast space target motion, and illumination changes. After the detection and tracking steps, the relative position, linear and angular velocities and attitude of space debris are estimated based on visual measurements. The projection of tracked feature points on two cameras creates an observation model of the filters, and the structure of a non-cooperative spacecraft is determined by estimating feature point positions. The relative attitude estimation is derived from the Modified Rodrigues Parameters (MRPs). Furthermore, to achieve minimal estimation errors, data from multiple sensors was integrated. To increase the system accuracy and manage difficulties associate with high uncertainty and noises associate with space navigation, two novel adaptive frameworks were proposed for the sensor fusion step: the first is a Fuzzy Adaptive Unscented Kalman Filter (FAUKF), and the second approach is an Adaptive Robust $\mathrm{H}_{\infty}$ Extended Kalman Filter (AHEKF). To protect filters from divergence when there is high initial uncertainty in covariance matrices and non-zero mean noise, adaptive factors are designed to tune the covariance matrix $P_{k}, R_{k}$ and $Q_{k}$. The accuracy of both proposed approaches, FAUKF and AHEKF, in terms of lower estimation error and higher precision are evaluated and compared with Extended Kalman Filter, Unscented Kalman filter and $\mathrm{H}_{\infty}$ Extended Kalman filter. It should be noted that the difference between the FAUKF algorithm developed for sensor fusion and the approach developed for target tracking is that here two fuzzy mechanisms were designed to manage white noise and 
high initial uncertainty separately, while the tracking step used one Fuzzy mechanism. Furthermore, the tuning parameters were optimized based on the application. Accurate evaluation of the proposed approaches requires experimental data from an appropriate testbed to create an environment that can precisely reproduce the types of motions and conditions that occur in actual space missions. This thesis employs the testbed at the spacecraft robotics and control laboratory of the Carleton University to accurately model spinning motions and complex environmental conditions, including intense illumination, blueness and noise. The testbed has computer vision-based navigation capability, through stereo cameras that can assess an unknown target object spinning about its major axis. In addition, actual space-based footage captured during the discovery space shuttle mission STS-131 is used. These datasets include ground truth measurements of both the inspecting spacecraft and the target object. 


\section{Acknowledgments}

I would like to express my sincere gratitude to Professor Jurek. Z Sasiadek for allowing me to conduct this research under his supervision. I am especially grateful for his confidence and the freedom he gave me to do this work, and Professor Steve Ulrich for his support in different stages of this work.

I would also like to show gratitude to my committee, including Professor DanSorin Necsulescu, Professor Jie Liu, Professor Mohamed Atia and Dr. Amirhossein Monjazeb for their time, comments and valuable feedback.

Most importantly, none of this could have happened without my family. My thanks go to my parents for their confidence and their love during all these years. I am, really appreciative and thankful for everything you have given me. I would especially like to express my gratitude to my husband, who has always supported me and helped me overcoming the difficulties without complaining. I would like to extend my warmest thanks to my dear daughter, Tara. If this work has sometimes prevented us from sharing important moments of life, know that I never stopped thinking about you. 


\section{Table of Contents}

Abstract $\quad$ iii

Acknowledgments $\quad$ vi

Table of Contents vii

List of Tables $\quad$ xii

List of Figures $\quad$ xiii

Nomenclature $\quad$ xvii

List of Symbols $\quad$ xix

1 Introduction 1

1.1 Motivation ........................... 1

1.2 Problem Statement . . . . . . . . . . . . . . . . 2

1.3 Literature Review . . . . . . . . . . . . . . . . . . . . . 3

1.3.1 Global Positioning System . . . . . . . . . . . . . 6

1.3.2 Lasers . . . . . . . . . . . . . . . . . . 7

1.3.3 Vision Sensors . . . . . . . . . . . . . . . . 8

1.3.4 Stochastic Filters . . . . . . . . . . . . . . . 14

1.4 Research Objectives . . . . . . . . . . . . . . . . . . . . 19 


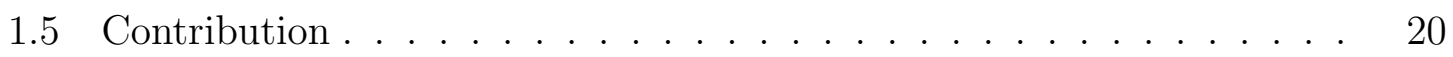

1.6 Outline of This Thesis . . . . . . . . . . . . . . . . . . . . 22

2 Theoretical Background $\quad 24$

2.0.1 Mathematical Model of a Pinhole Camera . . . . . . . . . 24

2.0.2 Mathematical Model of a Stereo Camera . . . . . . . . . 26

2.0 .3 Stereo Camera Calibration . . . . . . . . . . . . . . . . 27

2.1 Relative Translational Motion . . . . . . . . . . . . . . . . . . . . . 29

2.1.1 Clohessy Wiltshire Equations _. . . . . . . . . . . 30

2.1.2 Unpertubated Non-linear Model (Keplerian) . . . . . . . . . . 31

2.2 Rotational Motion . . . . . . . . . . . . . . . . . 32

$2.2 .1 \quad$ Euler Angles . . . . . . . . . . . . . . . . . . . . . . . . 33

2.2 .2 Quaternions . . . . . . . . . . . . . . . . 35

2.2.3 Modified Rodrigues Parameters . . . . . . . . . . . . . 37

2.2 .4 Angular Velocity . . . . . . . . . . . . . . . . . . . 38

2.2.5 Euler Equations for Torque-free Motion . . . . . . . . . . . 40

2.3 Bayesian Framework for State Estimation _ . . . . . . . . . 42

2.3.1 State Estimation . . . . . . . . . . . . . . . . 44

2.3 .2 Estimators . . . . . . . . . . . . . . . . . . . . . 45

2.3 .3 Dynamical System . . . . . . . . . . . . . . . . 46

2.3.4 Extended Kalman Filter _ . . . . . . . . . . . . . 48

2.3.5 Iterated Extended Kalman Filter _ . . . . . . . . . . . 52

2.3.6 Unscented Kalman Filter . . . . . . . . . . . . . . . . . . 54

2.4 Fuzzy Logic . . . . . . . . . . . . . . . . . . . 56

2.4 .1 Membership Function . . . . . . . . . . . . . . . . 57

$2.4 .2 \quad$ Fuzzy Rules . . . . . . . . . . . . . . . . . . 57 
2.4 .3 Defuzzification . . . . . . . . . . . . . . 58

3 Uncooperative Space Target Detection and Tracking 60

3.1 Introduction . . . . . . . . . . . . . . . . . 61

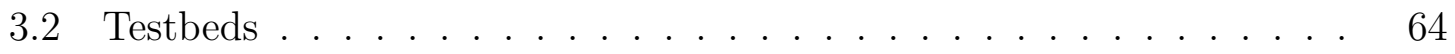

3.2.1 Facility at the Spacecraft Robotics and Control Laboratory of Carleton University. . . . . . . . . . . . . . . . . 64

3.2.2 Actual TriDAR Images During STS-131 Space Shuttle Mission 67

3.3 Target Detection . . . . . . . . . . . . . . . . . 70

3.3.1 Background Subtraction . . . . . . . . . . . . . 71

3.3.2 Feature Detection . . . . . . . . . . . . . . . . 71

3.3.3 Stereo Matching and 3-D Reconstruction . . . . . . . . . . 76

3.3.4 Disparity Map and Triangulation . . . . . . . . . . . . . . 77

3.4 Tracking . . . . . . . . . . . . . . . . . . . . 81

3.4.1 System Description . . . . . . . . . . . . . . 83

3.4.2 EKF and UKF for Feature Tracking . . . . . . . . . . . . . 84

3.4.3 Fuzzy Adaptive UKF . . . . . . . . . . . . . . 86

3.5 Experimental Results . . . . . . . . . . . . . . . . . . 94

3.5.1 Using Testbed at the Spacecraft Robotics and Control Laboratory of Carleton University . . . . . . . . . . . . 94

3.5.2 Using Actual Images from STS-131 Mission . . . . . . . . . . 101

4 Space Target's Pose and Motion Estimation 107

4.1 Introduction . . . . . . . . . . . . . . . . . . 107

4.2 Reference Frames . . . . . . . . . . . . . . . . . . . . . . . . . . . . . 110

4.2.1 Standard Earth-centered Inertial (ECI) . . . . . . . . . . . . . 110

4.2.2 Orbital Reference Frame . . . . . . . . . . . . . . . . . . 110 
4.2.3 Chaser Body-fixed Reference Frame . . . . . . . . . . . . . . 111

4.2.4 Stereo-vision-fixed Reference Frame . . . . . . . . . . . . . . 111

4.2.5 Target Body-fixed Reference Frame . . . . . . . . . . . . . . 111

4.3 Estimation Methodology . . . . . . . . . . . . . . . . . . 111

4.3.1 Dynamical Model . . . . . . . . . . . . . . . . . . . 112

4.3 .2 Process Model . . . . . . . . . . . . . . . . . . . 112

4.3.3 Relative Translational Dynamics . . . . . . . . . . . . . . 113

4.3.4 Relative Rotational Dynamics . . . . . . . . . . . . . . . . 114

4.3.5 Kinematically Coupled Spacecraft Relative Motion Model . . . 117

4.3.6 Observation Model . . . . . . . . . . . . . . . . . . . 121

4.4 Algorithm Summary . . . . . . . . . . . . . . . . 123

4.5 Simulation Experiment . . . . . . . . . . . . . . . . . 124

4.5.1 Results . . . . . . . . . . . . . . . . . . 127

5 Sensors Fusion $\quad 131$

5.1 Introduction . . . . . . . . . . . . . . . . . . 132

5.2 System Description . . . . . . . . . . . . . . . . . . 134

5.3 Fuzzy Adaptive Filters . . . . . . . . . . . . . . . . . . . . . . 134

5.3.1 Weighted Iterated Extended Kalman Filter . . . . . . . . . . . 135

5.3.2 Weighted Unscented Kalman Filter . . . . . . . . . . . . . . 137

5.4 Fuzzy Logic Controllers . . . . . . . . . . . . . . . . . . 139

5.4.1 Fuzzy Logic Adaptive Mechanism for Parameter Uncertainty . 140

5.4.2 Fuzzy Logic Adaptive Mechanism for Non-white Process Noise 143

5.5 Robust Extended Kalman Filter . . . . . . . . . . . . . . . . . 146

5.5.1 Adaptation of Error Covariance . . . . . . . . . . . . . . . . . 149

5.5.2 Adaptation of State and Measurement Covariance Matrices . . 151 
5.6 Simulation Experiment . . . . . . . . . . . . . . 153

5.6.1 Results...................... 157

5.6.2 Scheme 1:Robustness Against High Initial Uncertainties . . . . 158

5.6.3 Scheme 2: Robustness Against Non-zero Mean Noise . . . . . 160

5.6.4 Scheme 3: Robustness Against Non-zero Mean Noise and High Initial Uncertainties . . . . . . . . . . . . . . . . 168

5.6.5 Scheme 4: Robustness Against Measurement Outlier Data . . 170

6 Conclusion

List of References

Appendix A

202

A.1 GPS Satellite Geometry . . . . . . . . . . . . . . . 202

A.1.1 Error sources of GPS . . . . . . . . . . . . 205 


\section{List of Tables}

2.1 Fuzzy operations for AND and OR operators. . . . . . . . . . 58

2.2 Possible Fuzzy rules combination. . . . . . . . . . . . . . . . 58

2.3 The most well-known defuzzification methods. . . . . . . . . . . . 59

3.1 ZED stereo camera specifications. . . . . . . . . . . 65

3.2 TriDAR specifications. . . . . . . . . . . . . . . . 68

3.3 Tracking with EKF . . . . . . . . . . . . . . . . . 85

3.4 Tracking with $\mathrm{UKF} \ldots \ldots \ldots$. . . . . . . . . . 86

3.5 Rule table of $\alpha . \ldots \ldots$. . . . . . . . . . . . . . . . . . . . . . . . 92

3.6 Rule table of scale. . . . . . . . . . . . . . . . . . . . . . . . . 94

3.7 The position RMS errors for different duration of occlusion. . . . . . 101

3.8 Computational complexity. . . . . . . . . . . . . . . . 101

4.1 Target pose and motion estimation using UKF. . . . . . . . . . . . 123

4.2 States' initial values. . . . . . . . . . . . . . . . . . . . 124

4.3 Feature point specifications in the simulation. . . . . . . . . . 125

5.1 Rule table of $\alpha$ for parameter uncertainty $[1,2]$. . . . . . . . . . . . 142

5.2 Rule table of scale for for parameter uncertainty $[1,2]$. . . . . . . . 143

5.3 Rule table of $\alpha$ for non-white noise $[1,2] \ldots \ldots$. . . . . . . . 145

A.1 Satellite parameters [3]. . . . . . . . . . . . . . . . . . . . . . 204

A.2 Error sources of GPS . . . . . . . . . . . . . . . 205 


\section{List of Figures}

1.1 Distribution of space debris in space (Credits: NASA) . . . . . . . . . 4

1.2 Objects in earth orbit evolution (Credits: US Space Surveillance Network). . . . . . . . . . . . . . . . . 6

2.1 Model of pinhole cameras. . . . . . . . . . . . . . . 25

2.2 Model of parallel stereo cameras. . . . . . . . . . . . . . 26

2.3 Stereo calibration. . . . . . . . . . . . . . . . . . . . . 29

2.4 Extrinsic camera calibration . . . . . . . . . . . . . . . . . . . 29

2.5 Fuzzy Logic Controller. . . . . . . . . . . . . . . . . . 57

3.1 Testbed at the spacecraft robotics and control laboratory of Carleton University. . . . . . . . . . . . . . . . 66

3.2 TriDAR camera $[4] \ldots \ldots \ldots$. . . . . . . . . . . . 67

3.3 Neptec's TriDAR video footage during a docking maneuver with the ISS $(\mathrm{STS}-131) . \ldots \ldots \ldots \ldots$

3.4 TriDAR coordinate system. . . . . . . . . . . . . . . . 69

3.5 ISS coordinate system [5]. . . . . . . . . . . . . . . 70

3.6 Background subtraction. . . . . . . . . . . . . . 71

3.7 Detectors' performance for different lightening conditions. . . . . . . . 72

3.8 Detectors' performance for tumbling motion of the space target. . . . 73

3.9 Detectors' performance for different motion blur levels. . . . . . . . . 74 
3.10 Detectors' performance for different noise levels. . . . . . . . . . . . 75

3.11 Detectors' performance for different target's distances from the camera. 76

3.12 Matching and outlier removal. . . . . . . . . . . . . . . . . 77

3.13 Relative position errors. . . . . . . . . . . . . . . . 80

3.14 Relative velocity errors. . . . . . . . . . . . . . . . . . 80

3.15 Relative position at four different distances. . . . . . . . . . . . . . 81

3.16 Angular velocity error about the major axis of rotation. . . . . . . . . 82

3.17 The tracking process diagram. . . . . . . . . . . . . . . 83

3.18 Mean value membership functions. . . . . . . . . . . . . . . . . 91

3.19 Covariance membership functions. . . . . . . . . . . . . . . . . 91

$3.20 \alpha$ membership functions. . . . . . . . . . . . . . . . . . . . . 92

3.21 Surface plot of the fuzzy logic system. . . . . . . . . . . . . . 93

3.22 Experimental setup at Carleton University spacecraft and control laboratory. . . . . . . . . . . . . . . . . . . . . 95

3.23 Positions of feature points on the spinning target. . . . . . . . . . 98

3.24 Feature points' position errors for a spinning target. . . . . . . . . . 99

3.25 The trajectory path of the $i^{\text {th }}$ feature point during the spinning motion. 100

3.26 Measurement data. . . . . . . . . . . . . . . . . . . . . . . . 102

3.27 UKF motion estimation. . . . . . . . . . . . . . . . . . 102

3.28 FAUKF motion estimation. . . . . . . . . . . . . . . . . 102

3.29 Tacking samples, the first row from left to right the 300th frame, the 454th frame, the 531th frame, the second row from left to write the 586th frame, the 633th frame, the 669th, the third row from left to write, the 700th frame, the 757th frame, the 800th frame. (STS-131 mission, Neptec Group) . . . . . . . . . . . . . . . . . 103

3.30 Relative position errors of four feature points. . . . . . . . . . . . 104 
3.31 Measurement data. . . . . . . . . . . . . . . . . . 106

3.32 FAUKF motion estimation. . . . . . . . . . . . . . . 106

3.33 UKF motion estimation. . . . . . . . . . . . . . . . 106

4.1 Two rigid-body spacecraft reference frames. . . . . . . . . . . 118

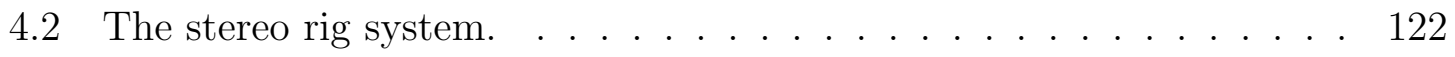

4.3 Reference and body frames. . . . . . . . . . . . . . . . 125

4.4 Relative position errors $[6] \ldots \ldots \ldots \ldots$. . . . . . . . . 127

4.5 Relative linear velocity errors $[6] \ldots \ldots \ldots$

4.6 Relative angular velocity errors $[6] . \ldots \ldots \ldots$

4.7 Quaternion errors [6]. . . . . . . . . . . . . . . . . . . . . . . . . . . . . . 129

4.8 Feature points position errors $[6] \ldots \ldots \ldots \ldots \ldots$

5.1 Mean value membership functions for parameter uncertainty $[1,2] \ldots 141$

5.2 Covariance membership functions for parameter uncertaint $[1,2] \ldots$. . 141

$5.3 \alpha$ membership functions for parameter uncertainty $[1,2] \ldots \ldots \ldots$

5.4 Mean value membership functions for non-zero mean process noise $[1$,

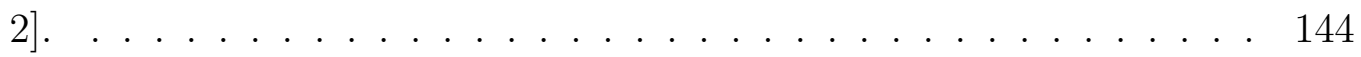

5.5 Covariance membership functions for non-zero mean process noise $[1,2] .144$

$5.6 \alpha$ membership functions for non-zero mean process noise $[1,2] \ldots \ldots 144$

5.7 Innovation sequences. . . . . . . . . . . . . . . . . . . 146

5.8 Block diagram representation of adaptive Kalman filters for GPS/INS

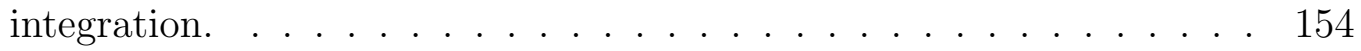

5.9 Variance of the filters for $5 \mathrm{Q}$ and $4 \mathrm{R} \ldots \ldots \ldots \ldots \ldots$

5.10 Variance of the filters for Q and 4R. . . . . . . . . . 159

5.11 Variance of the filters for $5 \mathrm{Q}$ and $\mathrm{R} \ldots \ldots \ldots \ldots \ldots \ldots$

5.12 Position and velocity errors comparison for $\mu=0.1 \ldots \ldots \ldots$

5.13 Position and velocity errors comparison for $\mu=0.5 . \ldots \ldots \ldots$ 
5.14 Position and velocity errors comparison for $\mu=1.5$. . . . . . . . . . 164

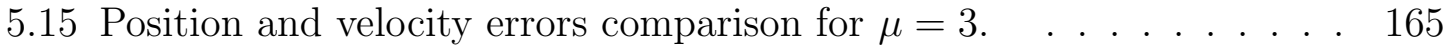

5.16 Position and velocity RMS errors comparison for $\mu=0.1 \ldots$. . . . 166

5.17 Position and velocity RMS errors comparison for $\mu=0.5 . \quad \ldots$. . . 166

5.18 Position and velocity RMS errors comparison for $\mu=1.5$. . . . . . . 167

5.19 Position and velocity RMS errors comparison for $\mu=3 . \quad$. . . . . . 167

5.20 Innovation sequences comparison. . . . . . . . . . . . . . . . . . 169

5.21 Position RMS errors comparison in X-direction, when both non-zero means and high-initial uncertainties are applied. . . . . . . . . . . 170

5.22 Velocity RMS errors comparison in X-direction, when both non-zero means and high-initial uncertainties are applied. . . . . . . . . . . 171

5.23 Position RMS errors comparison in Y-direction, when both non-zero means and high-initial uncertainties are applied. . . . . . . . . . . . 171

5.24 Velocity RMS errors comparison in Y-direction, when both non-zero means and high-initial uncertainties are applied. . . . . . . . . . . . 172

5.25 Position RMS errors comparison in Z-direction, when both non-zero means and high-initial uncertainties are applied. . . . . . . . . . . 172

5.26 Velocity RMS errors comparison in Z-direction, when both non-zero means and high-initial uncertainties are applied. . . . . . . . . . . 173

5.27 Position errors comparison against outlier measurement data. . . . . . 174

5.28 Velocity errors comparison against outlier measurement data. . . . . . 174

A.1 The WGS-84 coordinate reference frame $(x, y, z)$ used by GPS and a locally level coordinate reference frame $\left(x^{\prime}, y^{\prime}, z^{\prime}\right)[7] \ldots$. . . . . . . 203 


\section{Nomenclature}

$\begin{array}{ll}N_{L} & \text { chaser (leader) external torque } \\ N_{T} & \text { target external torque } \\ \boldsymbol{H} & \text { total angular momentum } \\ \boldsymbol{I} & \text { identity matrix } \\ \boldsymbol{I}_{L} & \text { inertia tensor of the chaser } \\ \boldsymbol{I}_{T} & \text { inertia tensor of the target } \\ \boldsymbol{F}^{i} & \text { feature vector } \\ i_{L} & \text { rotation quaternion } \\ e_{L} & \text { Modified rodrigues parameters } \\ \boldsymbol{\sigma} & \text { relative angular velocity } \\ \boldsymbol{D}(\boldsymbol{\sigma}) & \text { rotation matrix } \\ & \end{array}$




\begin{tabular}{|c|c|}
\hline$\dot{\vartheta}_{L}$ & chaser's orbital angular velocity \\
\hline$\ddot{\vartheta}_{L}$, & chaser's orbital acceleration \\
\hline$x$ & relative state vector \\
\hline$w$ & process noise \\
\hline$v$ & measurement noise \\
\hline$Q$ & state covariance matrix \\
\hline $\boldsymbol{R}$ & measurement covariance matrix \\
\hline$\lambda$ & scaling parameter \\
\hline$\alpha$ & tuning parameter \\
\hline$J$ & cost function \\
\hline$\gamma$ & threshold value \\
\hline$\delta$ & forgetting factor \\
\hline$U_{l}$ & lower limit criteria \\
\hline$U_{u}$ & upper limit criteria \\
\hline$\overline{\boldsymbol{V}}_{k}$ & residual \\
\hline $\boldsymbol{V}_{k}$ & innovation sequence \\
\hline $\boldsymbol{S}_{k}$ & theoretical covariance \\
\hline$\hat{C}_{v}$ & innovation covariance matrix \\
\hline
\end{tabular}

xviii 


\section{List of Symbols}

$\begin{array}{ll}\text { AHEKF } & \text { Adaptive } \mathrm{H}_{\infty} \text { Extended Kalman Filter } \\ \text { CoP } & \text { Center-of-Projection } \\ \text { CW } & \text { Clohessy Wiltshire } \\ \text { DGPS } & \text { Differential Global Positioning System } \\ \text { EKF } & \text { Extended Kalman Filter } \\ \text { ESA } & \text { European Space Agency } \\ \text { FAIEKF } & \text { Fuzzy Adaptive Iterated Extended Kalman Filter } \\ \text { FAUKF } & \text { Fuzzy Adaptive Unscented Kalman Filter } \\ \text { FLC } & \text { Fuzzy Logic Controller } \\ \text { GPS } & \text { Global positioning system } \\ \text { HEKF } & \text { H } \infty \text { Extended Kalman Filter } \\ \text { IEKF } & \text { Iterated Extended Kalman Filter } \\ \text { INS } & \text { Inertial Navigation Syetem } \\ \text { JAXA } & \text { Japan Aerospace Exploration Agency } \\ \text { KF } & \text { Kalman Filter } \\ \text { Low Earth Orbit }\end{array}$




$\begin{array}{ll}\text { MRP } & \text { Modified Rodrigues Parameters } \\ \text { NASA } & \text { National Aeronautics and Space Administration } \\ \text { rpm } & \text { revolutions per minute } \\ \text { sec } & \text { seconds } \\ \text { UKF } & \text { Unscented Kalman Filter } \\ & \\ \text { UT } & \text { Unscented Transform }\end{array}$




\section{Chapter 1}

\section{Introduction}

\subsection{Motivation}

Pose and motion estimation is the essential first step of any formation flight, including debris removal. Acquiring the estimates for high-speed spinning objects is difficult with most current algorithms. Extensive research on pose estimation of space targets has been conducted over the past few decades, and there have been some advances in the area. However, a practical algorithm that is robust under significant uncertainty and practical limitations, such as the abrupt motion of a target, extensive illumination and high uncertainty, requires further investigation and development. It is particularly important to track a target object and know it's pose and motion relative to the chaser spacecraft. If the target object is tumbling or spinning considerably, it is also

essential to know the linear and angular velocities. Most strategies in the literature assume prior knowledge of targets, while in reality, we rarely have information about a non-cooperative space target's motion and geometry. 


\subsection{Problem Statement}

This thesis develops new navigation methods to track and estimate the pose and motion of an unknown and uncooperative target, to capture and remove it. The stochastic framework is one of the widely used approaches for target tracking and poses estimation. However, due to the high uncertainty in real-time tracking of an uncooperative space target, its motion and the harsh conditions in space, using conventional approaches such as Extended Kalman Filter (EKF) or Unscented Kalman Filter (UKF) is highly challenging, and complicates the problem for many reasons.

The first complication is that the illumination in deep space can be very intense, and due to the abrupt motion, the space target can move in and out of the cameras' fields of view for a considerable period. These conditions make target tracking so challenging and sometimes nearly impossible. Target tracking is considered an essential step for pose and motion estimation; hence, in the case of missed or lost tracking data, pose estimation can not be conducted unless we can estimate the target states. Another complication associated with non-cooperative space target pose and motion estimation is that full state vector estimation of the target is required. Currently, we can only observe the position of the target, while other states must be calculated indirectly in some manner. Furthermore, high initial uncertainty and noise also contribute to the difficulty of achieving full state estimation. If we rely on one sensor only for localization, in case of sensor failure, the pose estimation algorithm is disturbed. This is why considering multiple sensors and integrating the data is a reliable approach.

With respect to the first complication, this thesis proposes a novel approach to improve orbital tracking performance for unknown and uncooperative space targets that are exhibiting fast motions and/or adverse environmental conditions. A new 
adaptive UKF for tracking feature points in video sequences is presented here. In this method, a fuzzy logic adaptation mechanism is employed to modify UKF characteristics, as well as noise factors. This approach estimates the location of the feature point and thereby the location of an unknown and uncooperative target. After the tracking step for pose and motion estimation, this thesis presents a filtering algorithm for relative state estimation based on visual navigation, which does not rely on markers on a target spacecraft. Finally, to manage the last complication the thesis presents new multi-sensor integration to fuse data from multiple sources. Due to the high initial uncertainty and noise levels, the conventional Kalman filter could fail to perform. To address this issue, two adaptive forms of Kalman filter are developed to provide reliable and accurate navigation solutions.

\subsection{Literature Review}

Over 100 spacecraft are launched every year, and historical analysis of the failure rate of satellites during their operational timeframe and the four to five percent failure rate of launch vehicles, means that approximately one of every seven satellites will fail before reaching their planned end of life [8]. There are more than 128 million pieces of debris orbiting the earth, at rates of up to several kilometers per second [9]; fast enough for a relatively small object to damage a satellite or a spacecraft. Space debris can be a danger to working space platforms such as the International Space Station, as well as spacecraft, shuttles and other manned vehicles. Figure. 1.1 gives an impression of space surrounding the earth and unlike what one might expect it shows a significant amount of space debris. Space debris can be due to collisions, deactivated or uncontrolled satellites, rocket parts and even lost tools. An analysis by NASA indicated that if all nations contributing to space debris, stopped launching 


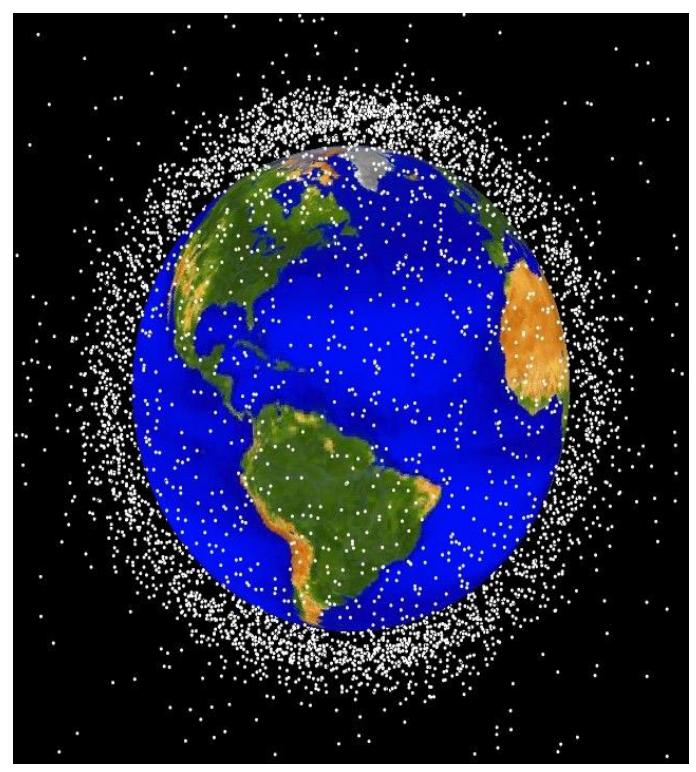

Figure. 1.1: Distribution of space debris in space (Credits: NASA)

missions, the number of objects in low earth orbit (LEO) would continue to increase for approximately 50 years due to collisions [10]. Thus, space debris removal is a critical and necessary undertaking.

Space debris are divided into three categories, according to their size. Category I $(<1 \mathrm{~cm})$ ranges from microscopic dust particles to objects $1 \mathrm{~cm}$ in diameter. The risk of damage from this category of debris is very low; hence, they are relatively harmless. Category II $(1-10 \mathrm{~cm})$ poses far greater risk than Category I. As of January 2019, about 900, 000 pieces of space junk orbiting the earth under $1500 \mathrm{~km}$ altitude in the 1 to $10 \mathrm{~cm}$ size range [11]. These objects are too small to be individually tracked but large enough to damage or destroy any craft they hit [12]. Category III (> 10 $\mathrm{cm})$ debris is mostly old satellites and the upper stage of launched rockets. It has been determined that this space debris category will increase due to collisions and explosions [12]. Therefore, removing these objects would reduce the amount of space debris in the long term and decrease the risk of collisions. NASA scientists determined that at least five massive objects, such as dead satellites or upper rocket stages, 
need to be de-orbited each year to reverse the current trend and stabilize the number of debris [13]. Figure. 1.2 shows number of objects $>10 \mathrm{~cm}$ in LEO. As shown in Figure. 1.2, recent events and human carelessness have dramatically increased the number of objects in LEO, particularly the Fengyun Chinese satellite break-up and the Iridium/Cosmos collision in 2007 and 2009. Many current and future in-orbit space operations involve automated physical interaction with dynamic, free-floating and free-flying targets. These operations include servicing satellites, capturing and disposal of space debris, and maintaining large space structures [14,15]. For these operations, it is critical to have precise knowledge of the target's position and attitude (pose), shape and dynamic model parameters. Moreover, to obtain a full navigation solution it is necessary to estimate the target's motion, as this is required for proper guidance and control of the chaser spacecraft. However, this information is usually unavailable, particularly in the case of damaged satellites, space debris, and thermally deformed or vibrating structures. Indeed, the relative navigation technology for uncooperative spacecraft is one of the key applications required for current and future space exploration. Relative navigation technology, and the corresponding sensors for autonomous navigation, have been studied and developed for several years. A number of approaches have been proposed to determine the relative attitude and orbit between spacecraft, and sensor types are chosen according to range and performance. The precision of relative navigation is maintained by navigation algorithms, and measurement sensors such as GPS, Laser Ranger Finder and vision sensors. 


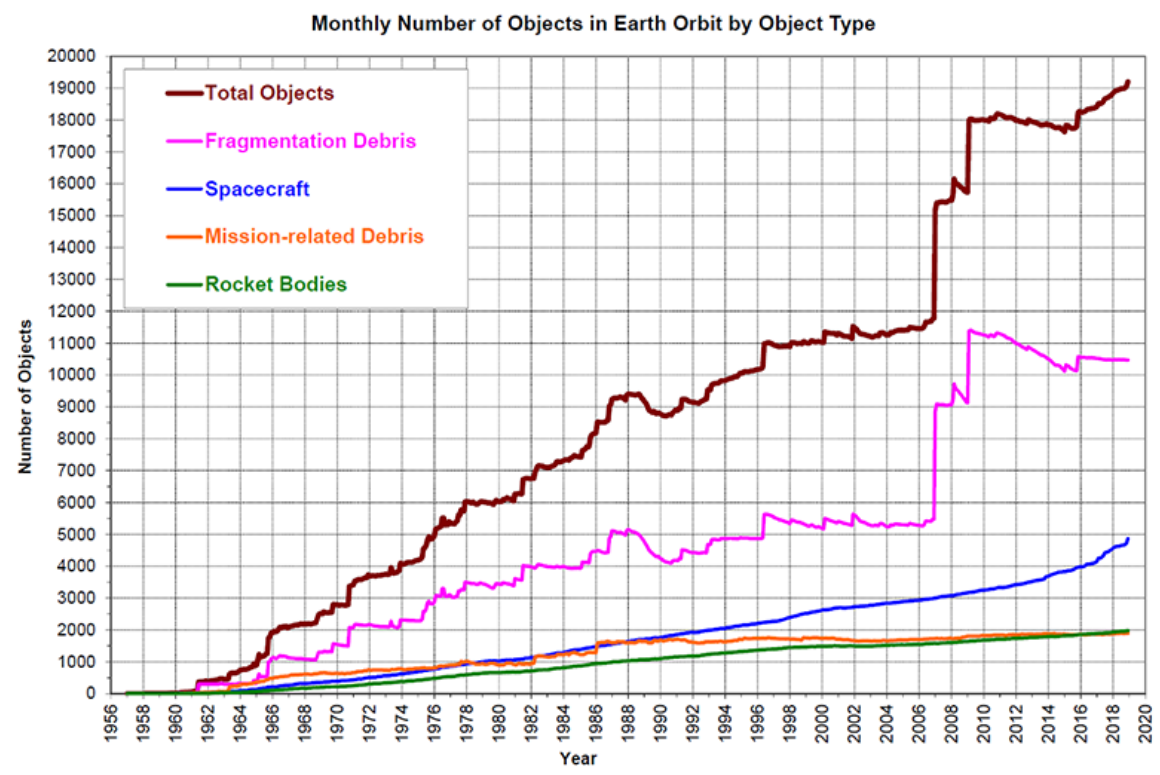

Figure. 1.2: Objects in earth orbit evolution (Credits: US Space Surveillance Network).

\subsubsection{Global Positioning System}

The Global Positioning System (GPS) is one of the well-known sensors used for space applications. Over the past two decades, spacecraft in LEO have significantly benefited from real-time reception of navigation and timing signals from GPS. By employing GPS receivers specially developed to support reception in space, LEO spacecraft now realize significantly reduced recovery time after trajectory maneuvers, improved operations cadence, increased satellite autonomy, and more precise, real-time navigation and timing performance. The European space agency's automated transfer vehicle uses two GPS receivers for navigation less than $250 \mathrm{~m}$ away from ISS. GPS is also used by Mohiuddin and Psiaki for relative navigation [16]. The single-frequency estimation techniques are used in their work, and GPS satellite residual position and clock error are integrated into EKF to estimate the relative states of high-altitude 
formation-flying spacecraft. The differential GPS (DGPS) was also used by Mohiuddin and Kawano $[17,18]$ to achieve relative navigation. Although the GPS can be used in many relative navigation problems, it is hard to use it for missions in which the GPS signal is difficult to obtain, such as High Earth Orbit (HEO) missions. An application of GPS-like technology to HEO has been proposed by Purcell [19]. More recently, there has been a transition from experimentation to operational utilization with reported usage by the United States [20], European Union [21], and Russia [22]. Two recent missions, MMS and GOES-16, employed GPS for accurate orbital positioning [23]. To manage challenges, such as week signal strength in HEO, special GPS receivers have been developed employing algorithms that enable the acquisition and tracking of weak signals from GPS satellites.

\subsubsection{Lasers}

Another well-known sensor used for many space missions is the laser range finder. The DARPA Orbital Express mission in 2007 verified several sensor technologies for short and long-range navigation. DARPA's Orbital Express mission used laser-based retro-reflectors for docking with a cooperative target spacecraft. A laser diode was used to illuminate a retro-reflective visual target that was processed by machine vision algorithms to obtain the relative position and orientation between the two spacecraft, the Advanced Video Guidance Sensor also used for docking between two spacecraft [24]. At the Japanese Aerospace Exploration Agency (JAXA), for the Hayabusa mission a set of laser altimeters was used to determine the spacecraft's altitude during the touchdown maneuver. However, due to laser sensor failures, this approach was abandoned during operations [25]. The Canadian Space Agency and NASA demonstrated the TriDAR (Triangulation and LIDAR) which is an automated rendezvous and docking sensing technology that measures distance by illuminating a 
target with a laser and analyzing the reflected light for performing relative navigation to an uncooperative (i.e. no fiducial) but known (i.e. using a geometric model) target [26]. The air force research laboratory and Lockheed Martin developed the XSS-11 mission. This small satellite is designed to quickly arrive at a space object and perform maneuvers around it without planning and minimal ground support. The vehicle used an active LiDAR and a star tracker for relative navigation [27]. However, LiDAR technology does not work well in areas or situations where there are high sun angles or huge reflections since the laser pulses depend on the principle of reflection. In general, he technology has limited applications in harsh environments. LiDAR is a technology that collects very huge datasets that require a high level of analysis and interpretation. For this reason, it may take a lot of time to analyze the data. Though the laser range finder sensors do not require a cooperating target, the relative navigation using scanning laser range finder would be challenging since relative distance should be small between the spacecraft, and its accuracy very depends on the reflectors.

\subsubsection{Vision Sensors}

A camera-based vision system has several advantages over other space application sensors. These include:

- cost and power benefits compared to active sensors;

- no need for a cooperating target (e.g. a receiver on the target or antennae);

- the measurement accuracy increases as the range decrease due to higher resolution;

- pose parameter measurements can be obtained in one shot;

- relative attitude measurements can be performed down to the minimum range; 
- the camera sensor has no moving parts, so it is less sensitive to launch and orbital environments;

- in principle, low range can be covered with sufficient FOV.

In the past, vision-based navigation systems using fiducial markers were successfully validated on different space missions. Jointly developed by Neptec and the Canadian Space Agency, cameras were used to monitor a pattern of distinct target dots on the object to be tracked. As an object moved, the system tracked the dots' changes, calculated the location and orientation of the object, and delivered this information to the operator in the form of graphical and textual cues. The information was used to position and orient a payload using Canadarm2, to join two objects in space station assembly [28]. A non-cooperative rendezvous is another challenge in space missions since preliminary knowledge of the target is deficient, and the typical measures for cooperative targets such as GNSS navigation cannot be used [29]. In addition, a non-cooperative target is not controlled, which means its attitude is in free motion in space. A chaser spacecraft must overcome these complications to establish accurate and reliable relative navigation. A visually based system for autonomous navigation is one of the best choices, but it is still a challenge for non-cooperative target spacecraft with no fiducial markers. Image processing is a key technology in autonomous navigation to extract high-precision, observable navigation data from a raw image. However, the on-orbit visual environment has two characteristics that make image processing difficult [30]: 1) intense, highly directional sunlight and 2) no diffusing background other than the earth. The intense sunlight characteristic causes very high image contrast, with the earth's albedo being the only diffuse light source. Since the target is non-cooperative, it is impossible to find the specific markers on its surface intended to assist image processing. For thermal protection, satellites 
are wrapped in Multi-Layer Insulation (MLI) materials, such as second surface aluminized Kapton (gold or specular), beta cloth (white or matte) and carbon-polyester coated Kapton (black or matte). Among these, aluminized Kapton is most commonly used and it gives the target a reflective surface and smooth edges. Because of these characteristics, optical features such as texture change according to the direction of lighting and view [30]; thus, efficient image processing techniques play an important rule. There are a few studies in the literature that address the problems of visual pose tracking of non-cooperative satellites, most of which assume the presence of a CAD model of the target satellite and use an iterative closest point (ICP) algorithm to register model and range data obtained from visual cameras [31]. Similarly, Miravet et al. [32] used a wire-frame model for camera-based tracking of an Orbital Life Extension Vehicle (OLEV). Estimating pose and dynamic parameters for guiding a robotic manipulator to capture a tumbling satellite, based on Neptec's laser-camera system and a CAD model, was experimentally demonstrated by Aghili et al. [33]. These approaches are suitable if prior knowledge about the geometry of the satellite and the initial pose are known, but without this information, the problem becomes more difficult. A vision-based approach using EKF was used to estimate relative pose and motion of non-cooperative targets [34]; however, the standard KFs such as the EKF cannot cope with high initial uncertainty and non-Gaussian white noise. Furthermore, it was assumed that feature points can be tracked in all frames. Of all visual systems, digital cameras are the optimal choice because unlike laser range finder, cameras sensors can be employed for tracking and inspection in different ranges. In addition, digital cameras are smaller and lighter than active devices, and they use less power. Most visual systems are based on monocular and binocular (stereo) systems. 


\section{Mono camera}

Various authors have identified monocular vision navigation as a capable technology for present and future formation-flying and on-orbit-servicing missions (e.g., PRISMA - Sweden [35], DEOS - Germany [36], PROBA-3 - ESA [37]). Augenstein et al. [38] presented a monocular approach, using an algorithm with SIFT and Fast simultaneous localization and mapping (SLAM) to estimate the pose and shape of an unknown target. Sonnenburg et al. [39] presented an SLAM-based EKF approach, including orbit dynamics to estimate relative kinematic states and the target structure. In both these approaches, the target was represented only by point clouds. However, because of the restricted mobility for exploration due to orbital dynamic constraints and unfavorable lighting conditions in space, only sparse and noisy point cloud data was provided by these algorithms. This creates a specific challenge for reconstruction algorithms due to the low information density of point clouds. Augenstein et al. [40] attempted to address the SLAM/SFM problem for a tumbling target with a static monocular camera. Their algorithm combined concepts from two existing approaches to pose tracking (Bayesian estimation methods and measurement inversion techniques) to estimate the relative pose between a robotic platform and a tumbling target in real-time. One problem was increased ambiguity in the orientation estimate, which has a non-convex cost function; the nonlinear minimization algorithm could converge to an incorrect local minimum. In addition, the center of mass of the target object with respect to the feature points was unknown. Du et al. [41] proposed a method based on two collaborating mono cameras sharing the recognition task to determine the pose of a large non-cooperative target. The main challenge with single-camera (monocular) applications is managing measurements that do not have sufficient information about the depth of a feature point. Depth information can be recovered using stereoscopic vision. 


\section{Stereo camera}

Stereo vision has been used in the past to estimate the relative state of two cooperative spacecraft. A camera-based 3-D feature tracking method using a stereo camera was presented by Oumer and Panin [42]. Their approach was based on a bank of Iterated Extended Kalman filters (IEKF), using point-wise kinematic models which feed the filtered state (depth) of the velocity estimator and vice-versa. To recover the velocity of a rigid body, they proposed a method to recover and track 3-D sparse structures, exploiting specular key points of the rigid target with the capability to predict features in the absence of image measurements. Lichter et al. [43] transformed simulated noisy 3-D points from stereo camera technology using computer graphic satellite images into voxels. In their work, rotational matrices and the centroid position of the principal geometry axes were derived, then used as coarse inputs to the Kalman Filter to estimate rotational and translational positions and velocities. Finally, shape estimation was achieved by solving the stochastic mapping problem. Abraham et al. [44] took stereo images of a miniature satellite model with a white matte surface and applied edge detection before stereo processing to create a sparse 3-D representation. Then the ICP algorithm was used to match a known model to the measured data points. Geometric probing combining a voxel template set and a binary decision tree was proposed by Jasiobedzki et al. [45] to address the potential for ICP to fall into a local minimum, depending on the initial relative attitude between the model and the measured data points. The algorithm used a combination of stereo vision and 3-D model matching applied to the ICP algorithm and a time series of images to increase the reliability of the relative attitude and position estimates. Tweddle [46] developed an algorithm based on the Incremental Smoothing and Mapping (iSAM) system for unknown and uncooperative objects that solves the SLAM problem for spacecraft proximity operation missions when the inspector state relative 
to an inertial frame is stationary or known, and the target object possibly spinning and rotating about any of its axes of inertia. A stereo camera-based Kalman filter for efficient recursive estimation of 3-D motion variables using a kinematics model-based approach was presented by Young and Chellappa [47]. However, these models were limited by continuous angular velocity and acceleration assumptions. Long et al. [48] proposed a binocular stereo-vision model and a non-cooperative target relative pose measuring method, based on stereo vision and RANSAC algorithm. A model-based pose refinement algorithm using a stereo camera, was presented by Kelsey et al. [49]. However, test results showed that their algorithm's performance depended on the initial pose estimate accuracy. A stereo camera-based pose estimation method without feature tracking was presented by Liu et al. [50]. Stereo vision was first used to reconstruct the target spacecraft, and a 3-D reconstruction was produced by merging the point clouds of each viewpoint. In this method a target-coordinate system was built using the reconstruction results, and then a point cloud registration algorithm was applied to solve the current pose between the observation spacecraft and target spacecraft. Rosso et al. [51] presented a stereo vision system architecture based on an FPGA device, tightly coupled programmable logic and a dual-core processor. Their work was improved by proposing a fast shape-from-shading algorithm [52]. Several real-time FPGA-based hardware implementations of stereo-vision algorithms have been proposed in the literature [53-55]. A relative pose estimation based on a stereo vision for the final phase of the rendezvous and docking of satellites was presented by Yu et al. [56]. The proposed estimation method used the relative attitude estimate based on TRIAD and QUEST to estimate the rotational states and the moment-ofinertia ratios. Currently, stereo-vision techniques are mainly used when dealing with 3-D video-based shape reconstruction, as they mimic the human visual system. 


\subsubsection{Stochastic Filters}

Though there has been much discussion about in-orbit maintenance and servicing of satellites and debris removal over the past several years, there is still not a practical method to reduce the threats to missions, operating satellites and the ISS. The first step in dealing with space debris is to detect the object and determine its pose and motion, in order to capture it for de-orbiting or repair. Efficient pose estimation that considers noise and other uncertainties is an open area for further research and development. In-orbit visual environment characteristics, including intense illumination and the earth background, change optical features such as texture according to the direction of light and the view. Thus, it is difficult to mimic such images with sufficient fidelity using computer graphics, which makes the development of robust estimation algorithms essential. Based on a literature review of various spacecraft pose estimations, it was determined that vision based sensors using stochastic filters, such as Kalman filters (KFs), improve pose estimation. An Extended Kalman filter-based algorithm for aircraft motion estimation from the optical-flow field of an onboard camera was suggested by Kendou [57]. A nonlinear EKF algorithm for spacecraft formation flight, using line-of-sight (LoS) vector measurement from the Visual Navigation Sensor coupled with gyro output, was presented by Kim et al. [58]. Psiaki [59] investigated double LoS measurement and the EKF for medium to long range autonomous rendezvous with a cooperative space target. The observation capability of the single LoS measuring system is less than that of the double (LoS) system, which also improves the relative position and velocity estimation accuracy. More than 800 research papers on topics related to robot's vision-based navigation using KFs have been published [60]. The Self-Tuning Kalman filter was proposed by Fung and Grimble [61], who developed a differential evolution for offline optimization. Papanikolopoulos [62] 
presented the Steady-State Kalman filter (SSKF) for visual tracking of a moving target, and the Ensemble Kalman filter for tomographic imaging of dynamic objects was suggested by Butala et al. [63]. The Switching Kalman filter (SKF) was developed by Chroust and Vincze [64] to cope with the delay introduced by image acquisition and processing, which is the main reason for limited tracking velocity and acceleration. Another Kalman filter, the Motor Extended-Kalman filter (MEKF), was introduced by Corrochano and Zhang [65]. The motor algebra for linearizing the 3-D Euclidean motion of lines was used as the theoretical basis for the development of this filter. The Hybrid Extended-Kalman Filter-based (HEKF) was developed to estimate robot position and orientation with a highly accurate model of a mobile robot in a large-scale environment [66]. Sharifi and Marey [67] proposed Iterative Adaptive EKF for pose estimation of an object in real-time by integrating mechanisms for noise adaptation and iterative-measurement linearization. The Kinematic Kalman filter (KKF) and Multi-Dimensional Kinematic Kalman filter (MD-KKF) were proposed by Jeon et al. [68]. The proposed method estimates the robot pose with a Recursive UKF, then the robot's pose was utilized to update landmark locations and enables the SLAM method to be performed in real-time. KKF and MD-KKF were proposed by Jeon et al. [69] for controlling industrial manipulators. By combining the measurements from a vision sensor, accelerometer, and gyroscope, MD-KKF recovers the inter-sample values and compensate for the measurement delay of the vision sensor providing the state information of the end-effector [70]. Tuning noise covariance of a Kalman filter for efficient object tracking using Particle-Swarm-Optimization-Aided EKF was proposed by Ramakoti, et al. [71]. Other proposed variations of EKF include DualUKF [72], Augmented State EKF [73], Modified Covariance EKF [74], Square-Root UKF [75], Fuzzy Logic Controller Kalman filter [76]. Handling uncertainty is an important problem for orbital relative navigation design, and significant uncertainties in 
the measurements, dynamics, and disturbances will occur with any formation. This is particularly true for orbital applications, where it is difficult to tune the filter for unexpected behavior manually. Among many different stochastic filters have been used for pose estimations, adaptive filtering is a powerful tool to cope with uncertainty and environment noise. A significant difficulty in designing standard KFs can often be due to incomplete a priori information about $\boldsymbol{R}_{k}$ and $\boldsymbol{Q}_{k}$ matrices. Incorrect a prior information can lead to divergence in KFs. Several schemes have been developed to address this problem; covariance setting was a traditional approach to improve performance. Einicke et al. [77] and Bolognani et al. [78] chose a positive definite system. An Adaptive EKF approach for online estimation of the covariance matrix was developed by Hoon et al. [79], Ahn and Won [80]. However, in some special cases, the optimal estimation of the covariance matrix can give an inaccurate approximation and affecting states estimate. A robust $\mathrm{H}_{\infty}$ filter was considered by Theodor et al. [81], Shen and Deng [82], Zhang et al. [83], Tseng and Chen [84]. Robust filters vary according to the types of disturbances they address, and the general performance criterion of these filters is to guarantee a bounded energy gain from the worst possible disturbance to the estimation error. Although the Robust EKF was extensively investigated by Einicke and White [85], Reif et al. [86] and Seo et al. [87], prescribing the level of disturbance attenuation is still an open problem. Xiong et al. [88] developed a Robust adaptive EKF to design an estimator based on the stability analysis and determine whether the error covariance matrix should be reset according to the magnitude of the innovation. Busse et al. [89] proposed a Robust EKF based on relatively simple measurement models and a linear Keplerian propagation model. The filter was adapted using the Modified Maximum Likelihood Estimator (MMLE) algorithm to increase its robustness to manage potential environment uncertainty. Jiancheng et al. [90] suggested an innovation-covariance estimation-based 
adaptive EKF algorithm when prior knowledge of the measurement noise is incorrect. An Adaptive EKF for the localization of mobile robots was developed by Jetto and Longhi [91]. In their work, the data from odometry and sonar sensors were combined through an Adaptive EKF, providing online estimates of robot position. Kim and Lee [79] proposed an adaptive two-stage EKF to estimate unknown fault bias in an INS-GPS loosely coupled system. An Adaptive Fading Extended Kalman Filter(AFEKF) was designed to develop the AEKF. The AFEKF can also be used for nonlinear stochastic systems when system information is only partially known. The Fuzzy Adaptive Equalizer with EKF adaptation was developed by Wong et al. [92], and an Adaptive Neuro-Fuzzy EKF for robot localization was introduced by Havangi et al. [93]. The Adaptive Neuro-Fuzzy attempts to estimate the elements of $\boldsymbol{Q}_{k}$ and $\boldsymbol{R}_{k}$ matrices of the EKF algorithm at each sampling instant during measurement update. An Adaptive EKF Using Artificial Neural Networks was developed by Stubberu et al. [94]. They designed a neuro-observer that can learn system uncertainty online; to enhance the overall performance of a control system with uncertainties in the stateestimator model. An Adaptive Fuzzy Strong Tracking EKF for GPS Navigation was developed by Jwo et al. [95]. Strong Tracking Kalman filter, which is essentially a nonlinear smoother algorithm that employs sub-optimal multiple fading factors, in which the softening factors are involved. An adaptive fuzzy terminal sliding-mode with EKF for control of MEMS z-axis gyroscope was observed in the work of Ghanbaria et al. [96]. An Adaptive UKF Algorithm for Mobile Robot Control was proposed by Song et al. [97]. The algorithm was designed to update the covariance of process uncertainties online by minimizing the cost function. The mechanism is intended to compensate for the lack of a priori knowledge of process uncertainty distribution and improve the performance of UKF. An adaptive UKF for target tracking with unknown process noise statistics was introduced by Shi et al. [98]. In this algorithm, 
to estimate the system process noise variance adaptively, modified Sage-Husa noise statistics estimator was introduced. The adaptive UKF can handle the recursive state filtering in the presence of unknown process noise covariance matrices. Cao et al. [99] proposed an adaptive UKF algorithm for process fault prognostics to improve the estimation of error covariance matrices. By introducing measurement innovation into the estimation of error covariance matrices, the proposed algorithm can compute the Kalman gain adaptively and make future measurement innovation uncorrelated. An adaptive UKF filtering algorithm for GPS position estimation, to estimate and adapt its covariance in real-time, was proposed by Liu and Lu. [100]. An Adaptive Fading UKF algorithm, based on the correction of process noise covariance, $\boldsymbol{Q}_{k}$, for the case of possible mismatches with the model, was introduced by Soken et al. [101].

\section{Adaptive fuzzy filters}

The Kalman filter requires all plant dynamics and noise processes to be known precisely, and the noise must be zero-mean white noise. Divergence occurs if the theoretical and actual behavior of the filter does not agree. The Adaptive fuzzy filter represents a filter, which is especially powerful in coping with nonlinearities and modeling errors since they do not require any mathematical model of the system. There are few papers on the application of Fuzzy logic to adapt the Kalman filter. Adaptive tuning of a Kalman filter via fuzzy logic for an intelligent autonomous underwater vehicle navigation system was used by Loebis et al. [102]. The Fuzzy logic was used for the online detection and correction of divergence in the single state Kalman filter. They used a method to adjust only the $\boldsymbol{R}_{k}$ matrix of both the Kalman filter and EKF. Related papers which implemented fuzzy logic to adapt the Kalman filter by correction of the single state are Kobayashi et al. [103], Longhi et at. [104] and Raimondi et at. [105]. An adaptive Fuzzy strong tracking EKF for GPS Navigation proposed by 
Jwo and Wang [95]. By monitoring the degree of divergence parameters based on the innovation information, a Fuzzy logic adaptive system was designed for dynamically adjusting the softening factor according to the change in vehicle dynamics. The Fuzzy interacting multiple model UKF for maneuvering vehicles was presented by Jwo and Tseng [84]. In their algorithm, the fuzzy logic controller aids the multiple interacting models, and by switching between filters, a suitable value for the process noise covariance can be determined. As a disadvantage, this method also requires more than one filter running simultaneously. Jwo et al. [106] proposed a fuzzy adaptive strong tracking unscented Kalman filter for ultra-tight GPS/INS integrated systems. To enhance the robustness of the UKF for process model uncertainty, a Strong Tracking Unscented Kalman filter (STUKF) was developed by directly extending the suboptimal fading factor used in the STF to the UKF $[107,108]$. The estimation performance of STUKF was improved by adaptively adjusting the sub-optimal fading factor by the fuzzy logic $[109,110]$. However, the STUKF has two major problems: 1) calculation of the sub-optimal fading factor requires cumbersome evaluation of the Jacobian matrix of system models, 2) the sub-optimal fading factor is incorporated in the entire filtering process, leading to loss of precision in the time segments without process model uncertainty.

\subsection{Research Objectives}

By providing an advanced solution for identifying the space targets, the primary objective of this research is to develop new tracking methods, pose and motion estimations of an uncooperative space target using a stereo vision system. Besides, due to the harsh space environment, different types of sensors are required. To integrate data from different sensors, two novel sensor fusion approaches are developed. 
In particular, the primary objective can be broken down into the following research objectives.

- To identify the gaps in the current state-of-the-art vision-based detection, tracking, pose and motion. estimation for spaceborne applications, and multi-sensor integration algorithms.

- To develop a target tracking approach to improve orbital tracking performance for unknown and uncooperative space targets that are exhibiting fast motion and adverse environmental conditions.

- To develop a feature-based pose estimation method that does not rely on markers on the target spacecraft and is more robust than the current state-of-the-art.

- To create multi-sensor integration algorithms to improve navigation performance and overcome challenges associated with current Kalman filter-based sensor fusion algorithms.

\subsection{Contribution}

Our main contributions in this dissertation, which are published and accepted in [111], [112], [113], [6], [2], and [114] can be summarized as follows:

- This dissertation introduces a novel, feature-based tracking algorithm explicitly developed for uncooperative space targets. The objective was to design a tracking algorithm that is robust and accurate fast motions of space debris and harsh environmental conditions. Thus, to enhance orbital target tracking capabilities, the study proposes an adaptive Unscented Kalman filter that can autonomously locate and track unknown space targets. To detect dynamic 
changes and prevent the UKF from divergence, a fuzzy logic adaptation mechanism is developed that can apply appropriate weighting factors to tune the filter.

- This work is based on visual navigation that addresses the issues of relative pose and motion estimation of an uncooperative spacecraft, using a stereo camera that does not require markers attached to the target surface. The full state vectors of the relative position, linear and angular velocities, attitude and feature point positions are estimated, and the structure of the non-cooperative spacecraft is determined by evaluating feature point positions. The relative attitude estimate is derived through the Modified Rodrigues Parameters.

- To achieve reliable and accurate navigation solutions in this dissertation two novel adaptive schemes for data fusion are proposed. The first integration algorithm is based on the combinations of the Fuzzy Logic Controllers (FLCs) and conventional KFs, including IEKF and UKF. The proposed algorithm is based on correcting both the process noise covariance matrix, $\boldsymbol{Q}_{k}$, and the measurement error covariance $\boldsymbol{R}_{k}$. FLCs are implemented to adjust the exponential weighting of the weighted UKF and IEKF, to protect the filters from divergence and improve navigational accuracy, specifically when the conventional filters fail to perform. It should be noted that the adaptive scheme developed for target tracking is different than the algorithm proposed for sensor fusion. For the target tracking, one fuzzy logic mechanism was introduced to apply the appropriate tuning parameters and adapt UKF, while the sensor fusion framework used two separate FLCs to manage the initial high uncertainty and white noise. Furthermore, to improve the performance, the tuning parameter was optimized differently for both applications. The second scheme is a novel Adaptive $\mathrm{H}_{\infty}$ 
Extended Kalman Filter (AHEKF). The suggested approach benefits from both robust and adaptive algorithms to overcome high uncertainties and noises while maintaining a desirable estimation accuracy. Two adaptive sections are considered. Firstly, an adaptive scale factor is designed, making the filter able to control the effect of non-zero mean noises. Secondly, an adaption of $\boldsymbol{R}_{k}$ and $\boldsymbol{Q}_{k}$ weights are considered to control the effect of high initial uncertainties and outliers. To evaluate the performances of the two proposed algorithms, three different schemes are assumed to verify the performance and robustness of the suggested approaches against high initial uncertainty, non-zero mean noises, and outlier data. Results obtained by the proposed FAUKF and AHEKF were compared with UKF, HEKF, EKF.

\subsection{Outline of This Thesis}

As Chapter 1 is now complete, the balance of the thesis begins at Chapter 2 with reviews of the theoretical background of this work. The camera model and calibration, orbital dynamic model, Bayesian frameworks for state estimations, and Fuzzy logic concepts are discussed. The chapter introduces the concepts, conventions and nomenclature required to understand the work in the following chapters. New contributions are not considered in this chapter.

Chapter 3 evaluates the state-of-the-art algorithms for feature detection, description, matching and outlier removal under various conditions that the visual system could encounter in deep space, and the best performing algorithms are identified. A new adaptation scheme of UKF to cope with the uncertainties in real-time tracking of an uncooperative space target is then introduced. The proposed tracking scheme 
can manage many of the difficulties of moving space targets, such as the disappearance of a feature point, complex space target motion, and illumination changes. The results on actual space-based footage and the testbed at the Spacecraft Robotics and Control Laboratory of Carleton University verified the robustness and effectiveness of the proposed tracking method.

Chapter 4 presents the algorithmic details of how rigid body dynamics can be used to achieve full state estimation of an uncooperative target relative to the chase spacecraft (i.e., relative positions, linear and angular velocities, and orientation).

Chapter 5 describes the adaptive mechanisms developed for multi-sensor fusion, and the proposed algorithms are evaluated for high initial uncertainty, different noise levels and outliers. The accuracy and convergence of the proposed approaches are then compared to conventional filters.

Finally, Chapter 6 presents the conclusion of this work. 


\section{Chapter 2}

\section{Theoretical Background}

This chapter provides a review of the background material necessary to explain the contributions of this thesis. While this chapter does not describe any specific contributions itself, it defines the terminology and conventions that will be used in the remainder of this thesis.

\subsubsection{Mathematical Model of a Pinhole Camera}

A pinhole camera is basically a single image plane that is exactly parallel with the pinhole. The image plane and the pinhole are separated by a distance $f$, which is known as the focal length. The geometry of the pinhole camera is illustrated in Figure. 2.1, and the 3-D point $\boldsymbol{\rho}_{w}=\left[x_{w}, y_{w}, z_{w}\right]$ in the world frame (Inertial frame) can be presented in the camera frame as below:

$$
\left[\begin{array}{l}
x_{C} \\
y_{C} \\
z_{C}
\end{array}\right]=\left[\begin{array}{ll}
\boldsymbol{R}_{C / w} & \boldsymbol{T}_{C / w}
\end{array}\right]\left[\begin{array}{l}
x_{w} \\
y_{w} \\
z_{w} \\
1
\end{array}\right]
$$


By having a rotation and translation between the world frame and the camera frame, $\left[\begin{array}{ll}\boldsymbol{R}_{C / w} & \boldsymbol{T}_{C / w}\end{array}\right]$, the mathematical model to describe the relationship between a 3-D point and its corresponding 2-D $(u, v)$ can be written as:

$$
s[v]=\left[\begin{array}{c}
f \\
f \\
1
\end{array}\right]=\left[\begin{array}{ccc}
0 & -c_{x} \\
0 & f & -c_{y} \\
0 & 0 & 1
\end{array}\right]\left[\begin{array}{ll}
\boldsymbol{R}_{C / w} & \boldsymbol{T}_{C / w}
\end{array}\right] \boldsymbol{\rho}_{w}
$$

where $s$ denotes the homogeneous scale factor. $\left(c_{x}, c_{y}\right)$ represents the optical center of the image plane. By substituting Equation (2.1) in the Equation (2.2), the corresponding 2-D $(u, v)$ can be given as:

$$
\begin{aligned}
& u=\frac{x_{w}}{z_{w}} f-c_{x} \\
& v=\frac{y_{w}}{z_{w}} f-c_{y}
\end{aligned}
$$

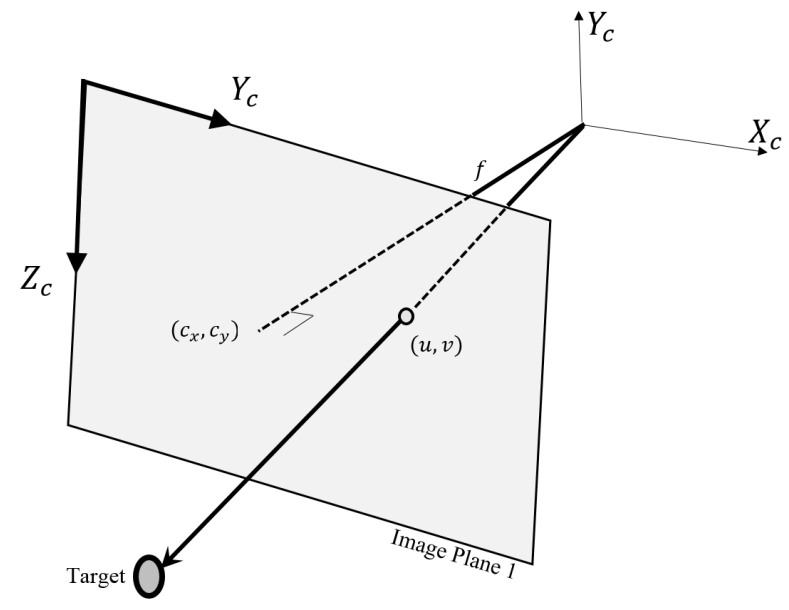

Figure. 2.1: Model of pinhole cameras. 


\subsubsection{Mathematical Model of a Stereo Camera}

A stereo camera consists of two parallel and aligned pinhole cameras that image the same point simultaneously. The cameras are separated by the baseline $\boldsymbol{D}_{R / L}=\boldsymbol{O}_{r}$ $\boldsymbol{O}_{l}$. Figure. 2.2 shows the geometry of a stereo camera, with $\boldsymbol{P}_{R}$ and $\boldsymbol{P}_{L}$ representing the projection of the point on the right and left image plans, respectively. This can be given by:

$$
\begin{aligned}
u_{r} & =\frac{x_{w}}{z_{w}} f_{r}-c_{r x} & v_{r} & =\frac{y_{w}}{z_{w}} f_{r}-c_{r y} \\
u_{l} & =\frac{x_{w}}{z_{w}} f_{l}-c_{l x} & v_{l} & =\frac{y_{w}}{z_{w}} f_{l}-c_{l y}
\end{aligned}
$$

where $\left(f_{r}, f_{l}\right)$ signifies the camera's right and left focal lengths.

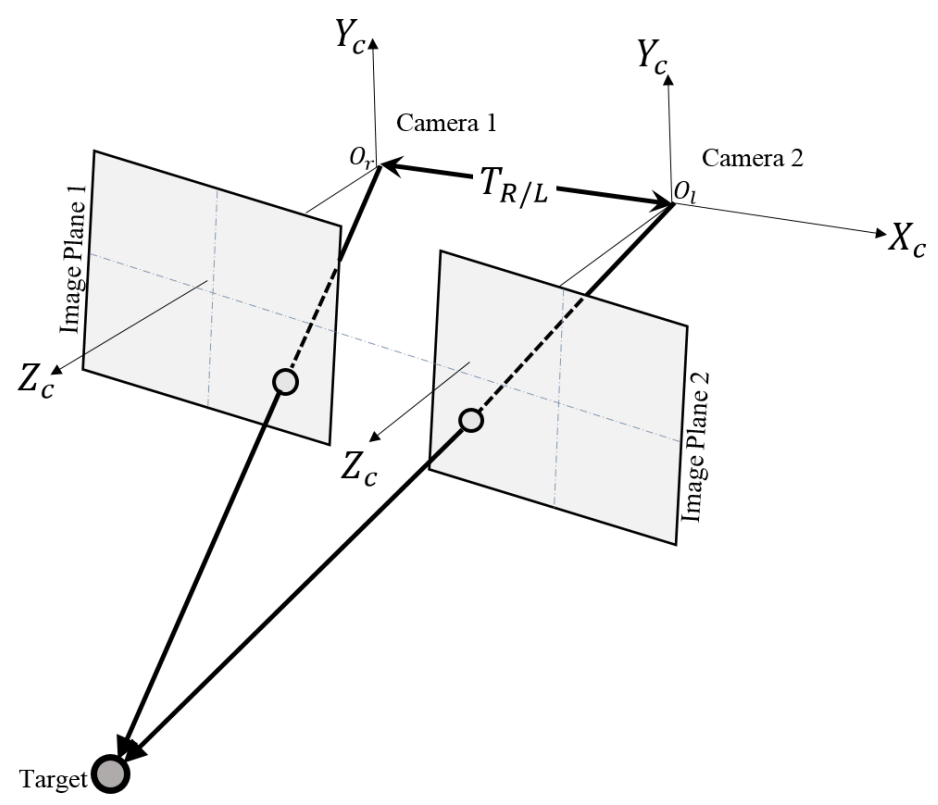

Figure. 2.2: Model of parallel stereo cameras. 


\subsubsection{Stereo Camera Calibration}

Camera calibration is required to estimate the parameters of the visual sensor lenses. The parameters can be used to correct lens distortions and for 3-D object reconstruction. The camera calibration calculates the intrinsic and extrinsic camera matrices. The intrinsic parameters include the transformation from the 3-D camera's coordinate to the 2-D image coordinate, and include the optical center $\left(c_{x}, c_{y}\right)$, focal length $\left(f_{x}, f_{y}\right)$ and skew coefficients $(s)$. This can be defined as:

$$
\boldsymbol{M}_{i n t}=\left[\begin{array}{ccc}
f_{x} & s & c_{x} \\
0 & f_{y} & c_{y} \\
0 & 0 & 1
\end{array}\right]
$$

The extrinsic parameters represent the rotation and translation from the 3-D world coordinate to the camera coordinate system.

$$
\boldsymbol{M}_{e x t}=\left[\begin{array}{cccc}
r_{11} & r_{12} & r_{13} & t_{1} \\
r_{21} & r_{22} & r_{23} & t_{2} \\
r_{31} & r_{32} & r_{33} & t_{3}
\end{array}\right]
$$

\section{Radial distortion}

All camera lenses have distortions, such as radial and tangential types. Radial distortion causes straight lines to be mapped as curved lines, particularly at the edges. The radial distortion can be modeled as follows:

$$
x_{\text {distorted }}=x_{\text {undistorted }}\left(1+k_{1} r^{2}+k_{2} r^{4}+k_{3} r^{6}\right)
$$




$$
\begin{gathered}
y_{\text {distorted }}=y_{\text {undistorted }}\left(1+k_{1} r^{2}+k_{2} r^{4}+k_{3} r^{6}\right) \\
r^{2}=\left(x_{\text {undistorted }}^{2}+y_{\text {undistorted }}^{2}\right)
\end{gathered}
$$

where $\left(k_{1}, k_{2}, k_{3}\right)$ represent the radial distortion coefficients of lenses. Normally, two coefficients are adequate for calibration, though for severe distortions such as wideangle lenses three coefficients can be used. It is noted that $k_{i}=0$ indicates the absence of radial distortion.

\section{Tangential distortion}

Tangential distortion happens when the image plane and the lens are not parallel. Tangential distortions are defined as follows:

$$
\begin{gathered}
x_{\text {distorted }}=x_{\text {undistorted }}+\left[2 * P_{1} * x_{\text {undistorted }} * y_{\text {undistorted }}+P_{2} *\left(r^{2}+2 * x_{\text {undistorted }}^{2}\right)\right] \\
y_{\text {distorted }}=y_{\text {undistorted }}+\left[P_{1} *\left(r^{2}+2 * y_{\text {undistorted }}^{2}\right)+2 * P_{2} * x_{\text {undistorted }} * y_{\text {undistorted }}\right] \\
r^{2}=\left(x_{\text {undistorted }}^{2}+y_{\text {undistorted }}^{2}\right)
\end{gathered}
$$

where $P_{1}$ and $P_{2}$ represent the distortion coefficients of a lens.

For the calibration step in this thesis, $(m=20)$ images were taken of a twelve-byseven chessboard grid with known geometry. The images were captured at varying positions and orientations to create an adequate set of viewpoints. Figure. 2.3 shows a photo of the checker-board, with the correspondence features highlighted by green circles.

The calibration process was done using OpenCV [115] and MATLAB, and the maximum likelihood was determined by minimizing the projection error with the least 


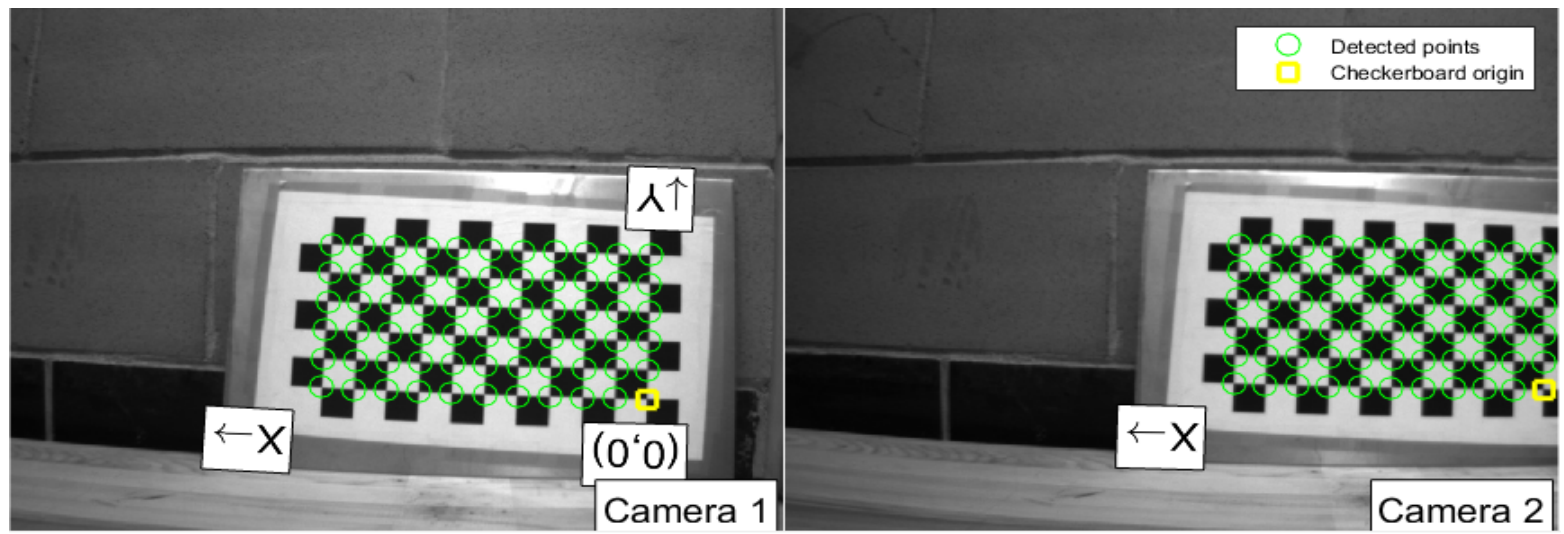

Figure. 2.3: Stereo calibration.
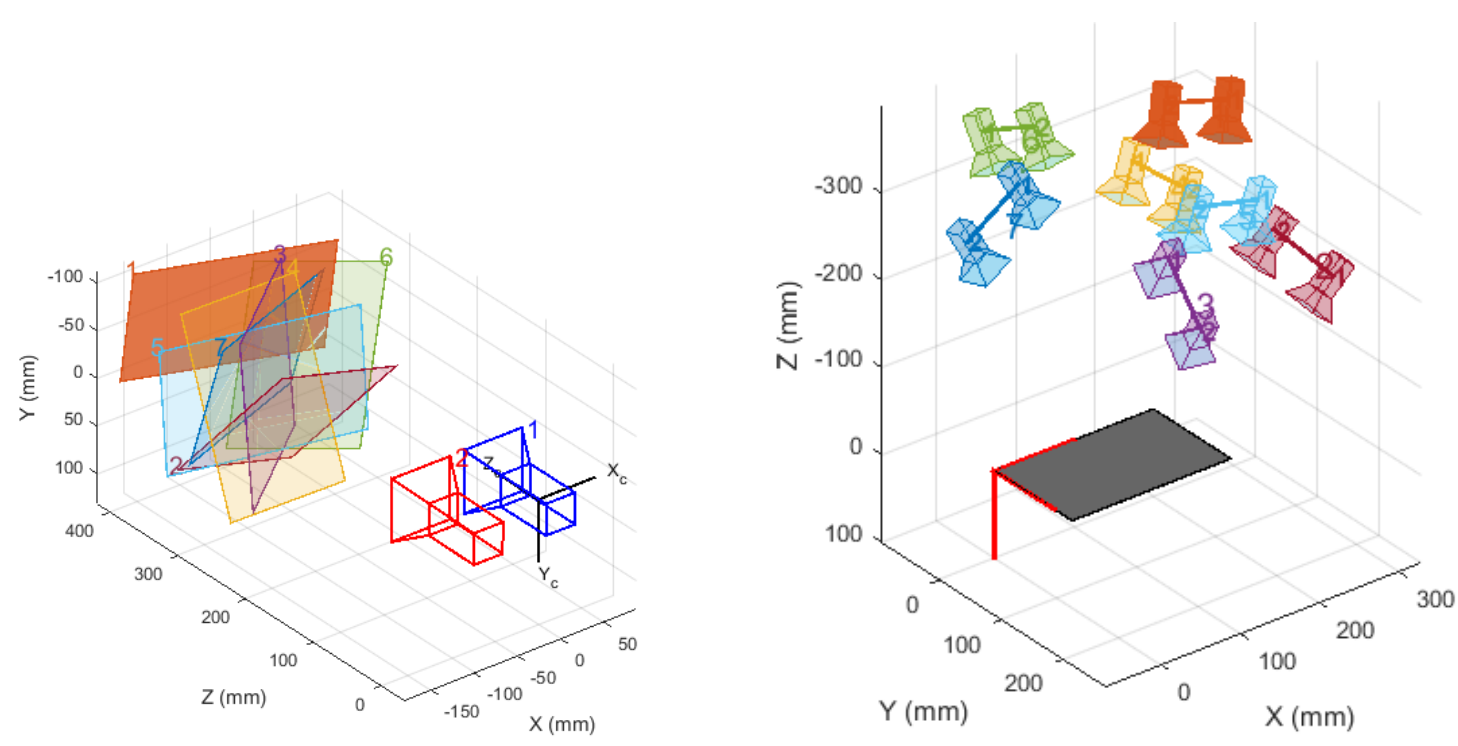

Figure. 2.4: Extrinsic camera calibration

square method. The stereo camera calibration using Matlab shows in Figure. 2.4.

\subsection{Relative Translational Motion}

Estimating the relative position and velocity between spacecraft is critical, particularly for long-term spacecraft formation; hence, deriving an accurate and simple dynamic model is an active research interest. Different orbital equations of motion are reported in the literature and classified into three main categories: the first is 
differential equation models (ODE), which are highly suitable for controller's design. The second category is typically described in terms of different orbital frames. Since this approach describes the formation geometry directly, it can be used to design spacecraft formation flights. The last category is a solution-based model.

\subsubsection{Clohessy Wiltshire Equations}

One of the early approaches is known as the Clohessy Wiltshire (CW) equations [116]. With this method, a set of linearized differential equations describes the relative motion of two spacecraft in a near-circular orbit:

$$
\begin{gathered}
\ddot{x}=2 n \dot{y}+3 n^{2} x \\
\ddot{y}=-2 n \dot{x} \\
\ddot{z}=-n^{2} z \\
n=\sqrt{\frac{\mu}{a_{t}^{3}}}
\end{gathered}
$$

where $\mu$ is the Earth's gravitational constant and $a_{t}$ is the semi-major axis of the target vehicle's orbit. The $z$-axis is the angular momentum vector of the target spacecraft, the $x$-axis is the radius vector and the $y$-axis completes the right-handed

system. With this definition, the central body is toward the negative $x$ direction, and the $y$-axis is along the velocity vector of the target spacecraft.

The CW equations have a closed-form solution, given by:

$$
\begin{gathered}
x(t)=(4-3 \cos (n t)) x_{0}+\frac{\sin (n t)}{n} \dot{x}_{0}+\frac{2}{n}(1-\cos (n t)) \dot{y}_{0} \\
y(t)=6(\sin (n t)-n t) x_{0}+y_{0}-\frac{2}{n}(1-\cos (n t)) \dot{x}_{0}+\frac{4 \sin (n t)-3 n t}{n} \dot{y}_{0}
\end{gathered}
$$




$$
z(t)=z_{0} \cos (n t)+\frac{\dot{z}_{0}}{n} \sin (n t)
$$

Though the CW equation has been successfully employed in some rendezvous missions, the error tends to accumulate over time. Thus, it is not suitable for long formation flights.

\subsubsection{Unpertubated Non-linear Model (Keplerian)}

The relative motion between two orbital bodies can be calculated with the Keplerian equations of motion. The reference orbit is the orbit of a chaser spacecraft, and the second orbit represents the orbit of the target spacecraft. The relative motion between the centers of mass of the target and chaser spacecraft can be written as:

$$
\begin{aligned}
& \overrightarrow{\boldsymbol{\rho}}=\overrightarrow{\boldsymbol{r}}_{T}-\overrightarrow{\boldsymbol{r}}_{C} \\
& \overrightarrow{\boldsymbol{r}}_{C}=-\frac{\mu}{\boldsymbol{r}_{C}} \overrightarrow{\boldsymbol{r}}_{C} \\
& \overrightarrow{\ddot{\boldsymbol{r}}}_{T}=-\frac{\mu}{\boldsymbol{r}_{T}} \overrightarrow{\boldsymbol{r}}_{T}
\end{aligned}
$$

Substituting Equations (2.19) and (2.20) into Equation (2.18) gives:

$$
\begin{gathered}
\overrightarrow{\boldsymbol{\rho}}=\frac{\mu}{\boldsymbol{r}_{C}+\boldsymbol{\rho}}\left(\overrightarrow{\boldsymbol{r}}_{C}+\overrightarrow{\boldsymbol{\rho}}\right)+\frac{\mu}{\boldsymbol{r}_{C}^{3}} \overrightarrow{\boldsymbol{r}}_{C} \\
\overrightarrow{\ddot{\boldsymbol{\rho}}}=\frac{d^{2} \overrightarrow{\boldsymbol{\rho}}}{d t^{2}}+2 \overrightarrow{\boldsymbol{\omega}} \times \frac{d \overrightarrow{\boldsymbol{\rho}}}{d t}+\frac{d \boldsymbol{\omega}}{d t} \times \overrightarrow{\boldsymbol{\rho}}+\overrightarrow{\boldsymbol{\omega}} \times(\overrightarrow{\boldsymbol{\omega}} \times \overrightarrow{\boldsymbol{\rho}})
\end{gathered}
$$

where

$$
\overrightarrow{\boldsymbol{\omega}}=\left(0,0, \dot{\vartheta}_{C}\right)^{T}
$$

where, $\boldsymbol{\rho}=[x, y, z]^{T}$ represents the relative position from the chaser's center of mass 
to the target's center of mass, expressed in the orbital coordinate frame. The relative velocity between the target and chaser is indicated as $\dot{\boldsymbol{\rho}}=[\dot{x}, \dot{y}, \dot{z}]^{T}$, and $\mu$ is the earth gravitational constant. The chaser's center of mass position, $r_{C}$, is given by:

$$
r_{C}=\frac{\left(1-e_{C}^{2}\right) a_{C}}{1+e_{C} \cos \vartheta_{C}}
$$

where, $a_{C}$ is the semi-major axis of the chaser's orbit, $\vartheta_{C}$ is the true anomaly, and $e_{C}$ represents the chaser's eccentricity. The orbital angular velocity $\dot{\vartheta}_{C}$, and the angular acceleration of the chaser $\ddot{\vartheta}_{C}$, are defined as:

$$
\begin{gathered}
\dot{\vartheta}_{C}=\sqrt{\frac{\mu}{a_{C}^{3}\left(1-e_{C}^{2}\right)^{3}}}\left(1+e_{C} \cos \vartheta_{C}\right)^{2} \\
\ddot{\vartheta}_{C}=\frac{-2 \dot{r}_{C} \dot{\vartheta}_{C}}{r_{C}}
\end{gathered}
$$

From the above equations, the relative translational equation of motion can be written as [117]:

$$
\begin{gathered}
\ddot{x}=2 \dot{\vartheta}_{C} \dot{y}+\ddot{\vartheta}_{C} y+\dot{\vartheta}_{C}^{2} x-\frac{\mu\left(r_{C}+x\right)}{\left[\left(r_{C}+x\right)^{2}+y^{2}+z^{2}\right]^{1.5}}+\frac{\mu}{r_{C}^{2}} \\
\ddot{y}=2 \dot{\vartheta}_{C} \dot{x}-\ddot{\vartheta}_{C} x+\dot{\vartheta}_{C}^{2} y-\frac{\mu(y)}{\left[\left(r_{C}+x\right)^{2}+y^{2}+z^{2}\right]^{1.5}} \\
\ddot{z}=-\frac{\mu(z)}{\left[\left(r_{C}+x\right)^{2}+y^{2}+z^{2}\right]^{1.5}}
\end{gathered}
$$

\subsection{Rotational Motion}

Determining the orientation and angular velocities of a spacecraft are essential for obtaining a complete navigation solution. The location and orientation together fully 
describe how the object is positioned in space.

\subsubsection{Euler Angles}

Euler angles are the first attempt to indicate orientation. It can be defined as rotation in terms of three consecutive rotations around the orthogonal axes . Euler determined that any rotation from one frame to another can be described by three rotations about the base vector. Euler angles are not unique, as there are twelve possible sets. Considering the rotation about $(x, y, z)$ axes by angles $(\phi, \theta, \gamma)$, we have [118]:

$$
\begin{aligned}
& \boldsymbol{R}_{x}(\phi)=\left[\begin{array}{ccc}
1 & 0 & 0 \\
0 & \cos (\phi) & -\sin (\phi) \\
0 & \sin (\phi) & \cos (\phi)
\end{array}\right] \\
& \boldsymbol{R}_{y}(\theta)=\left[\begin{array}{ccc}
\cos (\theta) & 0 & \sin (\theta) \\
0 & 1 & 0 \\
-\sin (\theta) & 0 & \cos (\theta)
\end{array}\right] \\
& \boldsymbol{R}_{z}(\gamma)=\left[\begin{array}{ccc}
\cos (\gamma) & -\sin (\gamma) & 0 \\
\sin (\gamma) & \cos (\gamma) & 0) \\
0 & 0 & 1
\end{array}\right]
\end{aligned}
$$

Spacecraft typically use the Tiat-Bryan angles orientation representation, known 
as yaw-pitch-roll, and the rotation matrix is defined as:

$$
\begin{gathered}
\boldsymbol{R}=\boldsymbol{R}_{z}(\gamma) \boldsymbol{R}_{y}(\theta) \boldsymbol{R}_{x}(\phi) \\
R=\left[\begin{array}{ccc}
\cos (\gamma) & -\sin (\gamma) & 0 \\
\sin (\gamma) & \cos (\gamma) & 0
\end{array}\right]\left[\begin{array}{ccc}
\cos (\theta) & 0 & \sin (\theta) \\
0 & 1 & 0 \\
0 & 0 & 1
\end{array}\right]\left[\begin{array}{ccc}
1 & 0 & 0 \\
0 & \cos (\phi) & -\sin (\phi) \\
-\sin (\theta) & 0 & \cos (\theta)
\end{array}\right]\left[\begin{array}{ccc}
0 & \sin (\phi) & \cos (\phi)
\end{array}\right]
\end{gathered}
$$

Although Euler angles can depict relative orientation, this approach is not effective for spacecraft rotational dynamics. Furthermore, due to the ambiguity of Euler angles, this method experiences singularities. For the Tiat-Bryan representation the singularity occurs when $\theta=\frac{\pi}{2}$.

\section{Euler parameter}

The Euler Parameter is another Euler contribution. The theorem states that instead of using three angles, the rotation motion can be determined by using one specific rotation specified as an angle of rotation $(\Phi)$ about a fixed axis [119]. This is known as the Euler axis of rotation or the Eigen axis, and is denoted by $\boldsymbol{\alpha}$. The rotation matrix in terms of the angle and axis of rotation can be defined as:

$$
\boldsymbol{R}=\cos (\Phi) \boldsymbol{I}+(1-\cos (\Phi)) \boldsymbol{\alpha} \boldsymbol{\alpha}^{T}-\sin (\Phi)\left[\boldsymbol{\alpha}^{\times}\right]
$$

where $\left[\boldsymbol{\alpha}^{\times}\right]$represents the cross product of a vector $\boldsymbol{\alpha}$.

Given the rotation matrix, the angle and axis of rotation can be found as:

$$
\Phi=\cos ^{-1}\left[\frac{1}{2}(\operatorname{trace}(\boldsymbol{R})-1)\right]
$$




$$
\boldsymbol{\alpha}^{\times}=\frac{1}{2 \sin (\Phi)}\left(\boldsymbol{R}^{T}-\boldsymbol{R}\right)
$$

The differential equation for the Euler angle using the spacecraft angular velocity can be defined as:

$$
\begin{gathered}
\dot{\Phi}=\boldsymbol{\alpha}^{T} \boldsymbol{\omega} \\
\dot{\boldsymbol{\alpha}}=\frac{1}{2}\left[\boldsymbol{\alpha}^{\times}-\cot \left(\frac{\Phi}{2}\right) \boldsymbol{\alpha}^{\times} \boldsymbol{\alpha}^{\times}\right] \boldsymbol{\omega}
\end{gathered}
$$

It is noted that kinematic singularity in Equations (2.38) and (2.39) is at $\Phi=0$ or $2 \pi$.

\subsubsection{Quaternions}

Unit Quaternion representation is one of the most popular ways of representing orientation in 3-D. It is represented by four parameters, as follows [120]:

$$
\boldsymbol{q}=\left[\begin{array}{l}
\boldsymbol{q}_{13} \\
q_{4}
\end{array}\right]
$$

with

$$
\boldsymbol{q}_{13}=\left[\begin{array}{l}
q_{1} \\
q_{2} \\
q_{3}
\end{array}\right]
$$

The matrix $\boldsymbol{q}_{13}$ forms the Euler axis component of the quaternion, and the parameter $q_{4}$ is a scalar parameter. The rotation matrix in terms of quaternion parameters 
can be written as:

$$
\boldsymbol{R}=\left(q_{4}^{2}-\boldsymbol{q}_{13}^{T} \boldsymbol{q}_{13}\right) \boldsymbol{I}+2 \boldsymbol{q}_{13} \boldsymbol{q}_{13}^{T}-2 q_{4} \boldsymbol{q}_{13}^{\times}
$$

The quaternion parameters can be express in terms of the elements of rotation matrix as:

$$
\begin{array}{r}
q_{4}= \pm \frac{1}{2} \sqrt{1+\operatorname{trace}(\boldsymbol{R})} \\
\boldsymbol{q}_{13}=\frac{1}{4 q_{4}}\left[\begin{array}{c}
R_{23}-R_{32} \\
R_{31}-R_{13} \\
R_{12}-R_{21}
\end{array}\right]
\end{array}
$$

Given the Euler parameters $(\Phi, \alpha)$, the four quaternion parameters can be written as:

$$
\begin{aligned}
\boldsymbol{q}_{13} & =\alpha \sin \left(\frac{\Phi}{2}\right) \\
q_{4} & =\cos \left(\frac{\Phi}{2}\right)
\end{aligned}
$$

With a spacecraft's angular velocity, the quaternion equation of motion is given by:

$$
\dot{\boldsymbol{q}}=\frac{1}{2} \boldsymbol{\Omega}(\boldsymbol{\omega}) \boldsymbol{q}=\frac{1}{2} \boldsymbol{\Xi}(q) \boldsymbol{\omega}
$$

where

$$
\boldsymbol{\Omega}(\boldsymbol{\omega})=\left[\begin{array}{ccc}
-\left[\boldsymbol{\omega}^{\times}\right] & \vdots & \boldsymbol{\omega} \\
\cdots & & \ldots \\
-\boldsymbol{\omega}^{T} & \vdots & 0
\end{array}\right]
$$




$$
\boldsymbol{\Xi}(\boldsymbol{q})=\left[\begin{array}{c}
q_{4} \boldsymbol{I}_{3 \times 3}+\left[\boldsymbol{q}_{13}^{\times}\right] \\
\ldots \ldots \ldots \\
-\boldsymbol{q}_{13}^{T}
\end{array}\right]
$$

where $\left[\boldsymbol{\omega}^{\times}\right]$and $\left[\boldsymbol{q}_{13}^{\times}\right]$are $3 \times 3$ dimensional matrices.

\subsubsection{Modified Rodrigues Parameters}

Quaternion representation is one of the most commonly used approaches for attitude estimation. However, because it uses four components to represent 3 DoF attitude motions, quaternion components are dependent, which leads to a constraint that the quaternion must have a unit norm. The constraint creates a singularity in the Kalman filter covariance matrix [121]. One approach to address this issue is to use the Modified Rodrigues Parameters [122].

A set of three attitudes can be obtained from the quaternion, as follows:

$$
\boldsymbol{\sigma}(\boldsymbol{q})=\frac{4}{1+q_{4}}\left[\begin{array}{l}
q_{1} \\
q_{2} \\
q_{3}
\end{array}\right]
$$

where, $\left[q_{1}, q_{2}, q_{3}, q_{4}\right]$ are four elements of the quaternion. And equivalently:

$$
\boldsymbol{q}(\boldsymbol{\sigma})=\frac{1}{16+\boldsymbol{\sigma}^{T} \boldsymbol{\sigma}}\left[\begin{array}{c}
8 \boldsymbol{\sigma} \\
16-\boldsymbol{\sigma}^{T} \boldsymbol{\sigma}
\end{array}\right]
$$

The differential equations that represent the attitude kinematics in terms of the 
MRPs can be derived as [123]:

$$
\begin{gathered}
\dot{\boldsymbol{\sigma}}=\frac{4}{1+q_{4}} \dot{\boldsymbol{q}}_{13}-\frac{4}{\left(1+q_{4}\right)^{2}} \dot{q}_{4} \boldsymbol{q}_{13} \\
=\left(-\frac{4}{1+q_{4}} \frac{1}{2}\left[\boldsymbol{\omega}^{\times}\right] \boldsymbol{q}_{13}+\frac{4}{1+q_{4}} \frac{1}{2} q_{4} \boldsymbol{\omega}\right)-\frac{4}{\left(1+q_{4}\right)^{2}}\left(-\frac{1}{2} \boldsymbol{\omega} \cdot \boldsymbol{q}_{13}\right) \boldsymbol{q}_{13} \\
=-\frac{1}{2}\left[\boldsymbol{\omega}^{\times}\right] \boldsymbol{\sigma}+\frac{1}{2} \frac{\boldsymbol{\omega} \cdot \boldsymbol{q}_{13}}{1+q_{4}} \boldsymbol{\sigma}+\frac{2 q_{4}}{1+q_{4}} \boldsymbol{\omega} \\
=\left(-\frac{1}{2}\left[\boldsymbol{\omega}^{\times}\right]+\frac{1}{8} \boldsymbol{\omega} \cdot \boldsymbol{\sigma}\right) \boldsymbol{\sigma}+\left(1-\frac{1}{16} \boldsymbol{\sigma}^{T} \boldsymbol{\sigma}\right) \boldsymbol{\omega}
\end{gathered}
$$

\subsubsection{Angular Velocity}

Torque applied to the object can be defined as:

$$
M=r \times \boldsymbol{F}
$$

where, $F$ denotes the force applied at a fulcrum point at a distance of $r$ from a rotation point. When the rotation point is located at the center of mass of an object, the torque is equal to the angular momentum change; hence, the equation 2.53 can be rewritten as:

$$
\boldsymbol{M}=\dot{\boldsymbol{H}}=\frac{d}{d t} \boldsymbol{H}
$$

where $\boldsymbol{H}$ defined as:

$$
\boldsymbol{H}=\boldsymbol{I} \boldsymbol{\omega}
$$

Applying the Coriolis theorems [124], the angular momentum of a rigid body can be found as:

$$
\boldsymbol{M}=\frac{d}{d t} \boldsymbol{H}+\boldsymbol{\omega} \times \boldsymbol{H}
$$


And substituting Equation (2.55) into the (2.56) gives:

$$
\begin{gathered}
\boldsymbol{M}=\frac{d}{d t}(\boldsymbol{I} \boldsymbol{\omega})+\boldsymbol{\omega} \times \boldsymbol{I} \boldsymbol{\omega} \\
\boldsymbol{M}=\boldsymbol{I} \dot{\boldsymbol{\omega}}+\boldsymbol{\omega} \times \boldsymbol{I} \boldsymbol{\omega}
\end{gathered}
$$

Which yields:

$$
\dot{\boldsymbol{\omega}}=-\boldsymbol{I}^{-1} \boldsymbol{\omega} \times \boldsymbol{I} \boldsymbol{\omega}+\boldsymbol{I}^{-1} \boldsymbol{M}
$$

where the $\boldsymbol{\omega}=\left[\begin{array}{lll}\omega_{x} & \omega_{y} & \omega_{z}\end{array}\right]^{T}$ is the angular rotation vector of a body around it's center of mass. $\boldsymbol{\omega}$ represents the inertia tensor of an object, and can be obtained by a symmetric matrix as follows:

$$
\boldsymbol{I}=\left[\begin{array}{ccc}
I_{x x} & I_{x y} & I_{x z} \\
I_{x y} & I_{y y} & I_{y z} \\
I_{x z} & I_{y z} & I_{z z}
\end{array}\right]
$$

Each elements of the inertia matrix can be found as [125]:

$$
\begin{gathered}
I_{x x}=\int_{m}\left(y^{2}+z^{2}\right) \mathrm{dm} \\
I_{y y}=\int_{m}\left(x^{2}+z^{2}\right) \mathrm{dm} \\
I_{z z}=\int_{m}\left(x^{2}+y^{2}\right) \mathrm{dm} \\
I_{x y}=\int_{m} x y \mathrm{dm}
\end{gathered}
$$




$$
\begin{aligned}
& I_{x z}=\int_{m} x z \mathrm{dm} \\
& I_{y z}=\int_{m} y z \mathrm{dm}
\end{aligned}
$$

Thus, Equation (2.59) can be rewritten as:

$$
\begin{aligned}
& \dot{\omega}_{x}=\frac{I_{y y}-I_{z z}}{I_{x x}} \omega_{y} \omega_{z}+\frac{1}{I_{x x}} M_{x} \\
& \dot{\omega}_{y}=\frac{I_{z z}-I_{x x}}{I_{y y}} \omega_{x} \omega_{z}+\frac{1}{I_{y y}} M_{y} \\
& \dot{\omega}_{z}=\frac{I_{x x}-I_{y y}}{I_{z z}} \omega_{y} \omega_{x}+\frac{1}{I_{z z}} M_{z}
\end{aligned}
$$

\subsubsection{Euler Equations for Torque-free Motion}

During torque-free motion, no external momentum (torque) is applied to the body. While the angular momentum is a constant, the angular velocity vector changes orientation with time. For torque free motion Equations (2.67 - 2.69) can be rewritten as:

$$
\begin{aligned}
& 0=I_{x x} \dot{\omega}_{x}-\left(I_{y y}-I_{z z}\right) \omega_{y} \omega_{z} \\
& 0=I_{y y} \dot{\omega}_{y}-\left(I_{z z}-I_{x x}\right) \omega_{z} \omega_{x} \\
& 0=I_{z z} \dot{\omega}_{z}-\left(I_{x x}-I_{y y}\right) \omega_{x} \omega_{y}
\end{aligned}
$$

Which yield

$$
\begin{aligned}
& \dot{\omega}_{x}=\frac{I_{y y}-I_{z z}}{I_{x x}} \omega_{y} \omega_{z} \\
& \dot{\omega}_{y}=\frac{I_{z z}-I_{x x}}{I_{y y}} \omega_{x} \omega_{z} \\
& \dot{\omega}_{z}=\frac{I_{x x}-I_{y y}}{I_{z z}} \omega_{y} \omega_{x}
\end{aligned}
$$


It should be noted that without an external moment, the dynamic is still active. In such a case, the angular velocity depends on the initial conditions and the principal axes of the angular velocity. If the magnitude of one angular velocity is much larger than two others, the linear stability analysis can perform small perturbations. In this case, the product of two quantities is small, as a result we can consider $\omega_{z}$ as a constant quantity. Therefore, the above equations can be rewritten as:

$$
\begin{gathered}
\dot{\omega}_{x}=\frac{I_{y y}-I_{z z}}{I_{x x}} \omega_{y} \omega_{z} \\
\dot{\omega}_{y}=\frac{I_{z z}-I_{x x}}{I_{y y}} \omega_{x} \omega_{z} \\
\dot{\omega}_{z}=0
\end{gathered}
$$

where $I_{y y}=I_{x x}$, and let consider $A=\frac{I_{y y}-I_{z z}}{I_{x x}} \omega_{z}$ then we have:

$$
\begin{gathered}
\dot{\omega}_{x}=A \omega_{y} \\
\dot{\omega}_{y}=-A \omega_{x}
\end{gathered}
$$

By differentiating both equations with time we have:

$$
\begin{aligned}
& \ddot{\omega}_{x}+A^{2} \omega_{x}=0 \\
& \ddot{\omega}_{y}+A^{2} \omega_{y}=0
\end{aligned}
$$

The solution to this differential equation for $\omega_{x}$ is:

$$
\omega_{x}(t)=B e^{\sqrt{A t}}+C e^{-\sqrt{A t}}
$$

The sign of $A$ determines the stability of the motion. If $A$ is negative, the motion is 
stable. This occurs when $I_{z z}$ is the major axis of inertia (i.e., $I_{z z}>I_{x x}$ and $I_{z z}>I_{y y}$ ) or when $I_{z z}$ is the minor axis of inertia (i.e., $I_{z z}<I_{x x}$ and $I_{z z}<I_{y y}$ ). When $A$ is positive, an exponential divergence will occur, and a minor initial condition $\omega_{x}$ can have unlimited growth. The conditions that lead to a positive $A$ are $I_{x x}<I_{z z}<$ $I_{y y}$ and $I_{y y}<I_{z z}<I_{x x}$. Thus, it can be concluded that the motion around the intermediate inertial is unstable, and rotation around either the major or minor axes is stable.

\subsection{Bayesian Framework for State Estimation}

The parameters and state of a physical system are never known precisely. Hence, the variables can characterize probabilistically in terms of random variables. Let us first recall some definitions of random variables.

\section{Probability density}

If $X$ is a random variable, then its Cumulative Distribution Function (CDF) is defined as:

$$
F_{X}(x)=P(X \leq x) \quad \forall \quad x
$$

where $x$ is non-random variable. The probability density, also known as the density of a continuous random variable, is a function that characterizes the statistical knowledge of a random variable. It is defined as a derivative of the probability distribution function as:

$$
p_{X}(x)=\frac{\mathrm{d} F_{X}(x)}{\mathrm{d} x}
$$

\section{Joint probability density}

With two random variables, $X$ and $Y$, their distribution functions are defined as: 


$$
\begin{aligned}
& F_{X}(x)=P(X \leq x) \\
& F_{Y}(y)=P(Y \leq y)
\end{aligned}
$$

The joint probability density of the two variables can be defined as the derivative of the joint probability density, as follows [126]:

$$
p_{X Y}(x, y)=\frac{\partial^{2} F_{X Y}(x, y)}{\partial x \partial y}
$$

where

$$
F_{X Y}(x, y)=P(X \leq x \cap Y \leq y)
$$

Random variables $X$ and $Y$ are independent if the joint probability function satisfies

$$
p_{X Y}(x, y)=p_{X}(x) \cdot p_{Y}(y)
$$

The joint probability density of two random variables can be extended to a random vector probability density. Thus, the multi-dimensional probability density of a random vector $\mathbf{X}=\left[X_{1}, X_{2}, \ldots, X_{m}\right]$ is defined as [126]:

$$
p(\boldsymbol{X})=p\left(x_{1}, x_{2}, \ldots, x_{m}\right)
$$

\section{Conditional probability density}

The conditional probability density function $p_{X}(x \mid y)$ represents the static knowledge of random variable $X$, given an observation of $Y$, and is given by [126]:

$$
p_{X}(x \mid y)=\frac{p_{X Y}(x, y)}{p_{Y}(y)}=\frac{p_{X Y}(x, y)}{\int p_{X Y}(x, y) d x}
$$


Thus we have:

$$
p_{X Y}(x, y)=p_{X}(x \mid y) p_{Y}(y)=p_{X}(x \mid y) \int p_{X Y}(x, y) d x
$$

Similarly

$$
\begin{gathered}
p_{Y}(y \mid x)=\frac{p_{X Y}(x, y)}{p_{X}(x)}=\frac{p_{X Y}(x, y)}{\int p_{X Y}(x, y) \mathrm{d} y} \\
p_{X Y}(x, y)=p_{Y}(y \mid x) p_{X}(x)=p_{Y}(y \mid x) \int p_{X Y}(x, y) \mathrm{d} y
\end{gathered}
$$

It should be noted that if the function $p_{X}(x \mid y)$ is known, and the measurement of random variable $Y$ provides information about random variable $X$, the conditional probability density is the primary tool for estimation.

\subsubsection{State Estimation}

Bayle's Theorem provides a mathematical solution for estimation. If there are two random variables $X, Y$, the posterior probability density function of $x, f_{X}(x \mid y)$ can be given by [126]:

$$
p_{X}(x \mid y)=\frac{p_{Y}(y \mid x) p_{X}(x)}{p_{Y}(y)}=\frac{p_{Y}(y \mid x) p_{X}(x)}{\int p_{Y}(y \mid x) p_{X}(x) \mathrm{d} x}
$$

In order to simplify the notations, $p_{X}(x)$ and $p_{X}(x \mid y)$ will denote by $p(x)$ and $p(x \mid y)$, respectively. Suppose a discretized system $F$ can be described by the vector $\boldsymbol{x}$ including the unknown states and a number of available measurements represented by a vector $\boldsymbol{d}$, using Bayes'rule we have [127]:

$$
p(\boldsymbol{x} \mid \boldsymbol{d})=\frac{p(\boldsymbol{d} \mid \boldsymbol{x}) p(\boldsymbol{x})}{\int p(\boldsymbol{d} \mid \boldsymbol{x}) p(\boldsymbol{x}) \mathrm{d} \boldsymbol{x}}
$$


where $p(\boldsymbol{x} \mid \boldsymbol{d})$ represents the posterior probability density function, and $p(\boldsymbol{d} \mid \boldsymbol{x})$ is the likelihood function. This function can be obtained directly from the measurement equation, which relates the measurement to the state and noise processes. $p(\boldsymbol{x})$ denotes the prior density function of random variable $X$. The main difficulty with the Bayes theorem is obtaining $\int p(\boldsymbol{d} \mid \boldsymbol{x}) p(\boldsymbol{x}) \mathrm{d} \boldsymbol{x}$ [127]. If $\boldsymbol{d}$ depends linearly on $\boldsymbol{x}$, and the measurement noise is Gaussian, the integral can be obtained analytically. The numerical approximation of this integral could be problematic, particularly when the dimension of $\boldsymbol{x}$ is increased. In the case of nonlinear and non-Gaussian noise the solution can only be approximated, and finding the most appropriate method for approximation can be very challenging.

\subsubsection{Estimators}

The estimator provides an estimate $\hat{\boldsymbol{x}}$ of $\boldsymbol{x}$. The Mean Squared Error Estimator is one of the well-known estimators acquired from the posterior pdf.

\section{Mean Squared Error}

The Mean Squared Error of an estimator is defined as the expected value of the squared error or deviation.

$$
M S E(\boldsymbol{x})=E\left[(\hat{\boldsymbol{x}}-\boldsymbol{x})^{2}\right]
$$

It indicates how close the collections of estimates are to the parameter being estimated. 


\section{Variance}

The variance is the expected value of the squared sampling deviation and is defined as:

$$
\operatorname{var}(X)=E\left[\boldsymbol{x}^{2}\right]-E[\boldsymbol{x}]^{2}
$$

It relates to the spread of the data set and indicates how far the collection of estimates are from their average value.

\subsubsection{Dynamical System}

States of a dynamic system iterative $[0, k]$ can be represented by:

$$
\boldsymbol{X}_{k}=\left[x_{0}, x_{1}, \ldots, x_{k}\right]
$$

Likewise the measurement vector on the same time intervals can be represented as:

$$
\boldsymbol{Y}_{k}=\left[y_{0}, y_{1}, \ldots, y_{k}\right]
$$

To derive the posterior pdf of $\boldsymbol{X}_{k}$, the Bayes rule can be applied as follows:

$$
p\left(\boldsymbol{X}_{k} \mid \boldsymbol{Y}_{k}\right)=\frac{p\left(\boldsymbol{Y}_{k} \mid \boldsymbol{X}_{k}\right) p\left(\boldsymbol{X}_{k}\right)}{\int p\left(\boldsymbol{Y}_{k} \mid \boldsymbol{X}_{k}\right) p\left(\boldsymbol{X}_{k}\right) \mathrm{d} \boldsymbol{X}_{k}}
$$

In terms of a requirement for recursive evaluation, the state at time $t_{k}$ depends only on the state of the preceding as:

$$
\boldsymbol{x}_{k}=f\left(\boldsymbol{x}_{k-1}, \boldsymbol{u}_{k}\right)+\boldsymbol{\omega}_{k}
$$

The random vector $\boldsymbol{\omega}_{k}$ denotes uncertainties in the model. It assumes zero-mean, 
white noise, random sequences with known covariances, all of which are uncorrelated with the initial state $\boldsymbol{x}_{0 \mid 0}$.

$$
E\left[\boldsymbol{\omega}_{k}\right]=0 \quad E\left[\boldsymbol{\omega}_{k} \boldsymbol{\omega}_{k}^{T}\right]=\boldsymbol{Q}_{k} \quad E\left[\boldsymbol{\omega}_{k} \boldsymbol{\omega}_{j}^{T}\right]=0 \quad \forall \quad k
$$

The measurement vector $\boldsymbol{Y}_{k-1}$ must be independent at different times. Their pdf is given by:

$$
p\left(\boldsymbol{y}_{k} \mid \boldsymbol{x}_{k}, \boldsymbol{Y}_{k-1}\right)=p\left(\boldsymbol{y}_{k} \mid \boldsymbol{x}_{k}\right)
$$

Under these assumptions, the marginal probability of $\boldsymbol{x}_{k}$ can be written as:

$$
p\left(\boldsymbol{x}_{k} \mid \boldsymbol{Y}_{k}\right)=\int p\left(\boldsymbol{X}_{k} \mid \boldsymbol{Y}_{k}\right) \mathrm{d} X_{k}
$$

The Equation (2.106) can be rewritten as:

$$
p\left(\boldsymbol{x}_{k} \mid \boldsymbol{Y}_{k}\right)=\frac{p\left(\boldsymbol{y}_{k} \mid \boldsymbol{x}_{k}\right) p\left(\boldsymbol{x}_{k} \mid \boldsymbol{Y}_{k-1}\right)}{p\left(\boldsymbol{y}_{k} \mid \boldsymbol{Y}_{k-1}\right)}
$$

Equation (2.107) is a very important relationship for recursive estimation, such as filtering problems. The filtering problem can be divided into two main steps, as follows:

Prediction step:

$$
p\left(\boldsymbol{x}_{k} \mid \boldsymbol{Y}_{k-1}\right)=\int p\left(\boldsymbol{x}_{k} \mid \boldsymbol{x}_{k-1}\right) p\left(\boldsymbol{x}_{k-1} \mid \boldsymbol{Y}_{k-1}\right) \mathrm{d} \boldsymbol{x}_{k-1}
$$

Analysis step:

$$
p\left(\boldsymbol{x}_{k} \mid \boldsymbol{Y}_{k}\right)=\frac{p\left(\boldsymbol{y}_{k} \mid \boldsymbol{x}_{k}\right) p\left(\boldsymbol{x}_{k} \mid \boldsymbol{Y}_{k-1}\right)}{p\left(\boldsymbol{y}_{k} \mid \boldsymbol{Y}_{k-1}\right)}
$$


where

$$
\begin{gathered}
p\left(\boldsymbol{y}_{k} \mid \boldsymbol{Y}_{k-1}\right)=\int p\left(\boldsymbol{y}_{k} \mid \boldsymbol{x}_{k}\right) p\left(\boldsymbol{x}_{k} \mid \boldsymbol{Y}_{k-1}\right) \mathrm{d} \boldsymbol{x}_{k} \\
E\left(\boldsymbol{v}_{k}\right)=0 \quad E\left[\boldsymbol{v}_{k} \boldsymbol{v}_{k}^{T}\right]=\boldsymbol{R}_{k} \quad E\left[\boldsymbol{v}_{k} \boldsymbol{v}_{j}^{T}\right]=0 \quad \forall \quad k
\end{gathered}
$$

where $\boldsymbol{v}_{k}$ represents the measurement noise. It is noted that if the random vectors $\boldsymbol{\omega}_{k}$ and $\boldsymbol{v}_{k}$ are uncorrelated, we have:

$$
E\left[\boldsymbol{\omega}_{k} \boldsymbol{v}_{k}^{T}\right]=0 \quad \forall \quad k, j
$$

The significant difficulty of filtering estimation is that the above integrals cannot be solved analytically. For exceptional cases, such as linear and state measurement with additive Gaussian Noise, there is a closed-form solution for $p\left(\boldsymbol{x}_{k} \mid \boldsymbol{Y}_{k}\right)$ using the Kalman filter. Otherwise, the first approach is to linearize the state and measurement model, which results in EKF. Also, if the second state variable is Gaussian, using determination sampling methods results in UKF.

\subsubsection{Extended Kalman Filter}

In EKF, the state transition and observation model functions can be non-linear rather than linear. The non-linear state and observation functions can be written as [128]:

$$
\begin{gathered}
\boldsymbol{x}_{k}=f\left(\boldsymbol{x}_{k-1}, \boldsymbol{u}_{k}\right)+\boldsymbol{\omega}_{k} \\
\boldsymbol{y}_{k}=h\left(\boldsymbol{x}_{k}\right)+\boldsymbol{v}_{k}
\end{gathered}
$$

Here $\boldsymbol{\omega}_{k}$ and $\boldsymbol{v}_{k}$ are the process and observation noises, and are assumed to be zero-mean Gaussian noises with covariance $\boldsymbol{Q}_{k}$ and $\boldsymbol{R}_{k}$, respectively. $\boldsymbol{u}_{k}$ is the control vector. 


\section{Initialization}

The only available information is the mean and covariance $\boldsymbol{P}_{0 \mid 0}$ of the initial state. Thus, the initial optimal estimate $\hat{\boldsymbol{x}}_{0 \mid 0}$ and error covariance can be written as:

$$
\hat{\boldsymbol{x}}_{0 \mid 0}=E\left[\boldsymbol{x}_{0}\right], \quad \boldsymbol{P}_{0 \mid 0}=E\left[\left(\boldsymbol{x}_{0}-\hat{\boldsymbol{x}}_{0 \mid 0}\right)\left(\boldsymbol{x}_{0}-\hat{\boldsymbol{x}}_{0 \mid 0}\right)^{T}\right]
$$

\section{Prediction}

With the optimal estimate $\hat{\boldsymbol{x}}_{k \mid k-1}$ and a covariance of $\boldsymbol{P}_{k \mid k-1}$, the predictable aspect can be obtained as:

$$
\hat{\boldsymbol{x}}_{k \mid k-1}=E\left[\boldsymbol{x}_{k} \mid \boldsymbol{Y}_{k-1}\right]=E\left[f\left(\boldsymbol{x}_{k-1}, \boldsymbol{u}_{k}\right)+\omega_{k} \mid \boldsymbol{Y}_{k-1}\right]
$$

Expanding $f($.$) in the Taylor series about \hat{\boldsymbol{x}}_{k-1 \mid k-1}$ we get:

$$
f\left(\boldsymbol{x}_{k-1}, \boldsymbol{u}_{k}\right)=f\left(\hat{\boldsymbol{x}}_{k-1 \mid k-1}, \boldsymbol{u}_{k}\right)+\boldsymbol{F}_{k} \boldsymbol{e}_{k-1}+\text { H.O.T }
$$

where $\boldsymbol{e}_{k-1}=\left(\boldsymbol{x}_{k-1}-\hat{\boldsymbol{x}}_{k-1 \mid k-1}\right)$, and the Jacobian matrix $\boldsymbol{F}_{k}$ is given by:

$$
\left.\boldsymbol{F}_{k} \approx \frac{\partial f}{\partial \boldsymbol{x}}\right|_{\hat{\boldsymbol{x}}_{k-1 \mid k-1, \boldsymbol{u}_{k}}}
$$

The Higher Order Terms (H.O.T) are negligible, so we have:

$$
f\left(\boldsymbol{x}_{k-1}, \boldsymbol{u}_{k}\right) \approx f\left(\hat{\boldsymbol{x}}_{k-1 \mid k-1}, \boldsymbol{u}_{k}\right)+\boldsymbol{F}_{k} \boldsymbol{e}_{k-1}
$$

The expected value of $f\left(\boldsymbol{x}_{k-1 \mid k-1}, \boldsymbol{u}_{k}\right)$ conditioned by $\boldsymbol{Y}_{k-1}$ :

$$
E\left[f\left(\boldsymbol{x}_{k-1}, \boldsymbol{u}_{k}\right) \mid \boldsymbol{Y}_{k-1}\right] \approx f\left(\hat{\boldsymbol{x}}_{k-1 \mid k-1}, \boldsymbol{u}_{k}\right)+\boldsymbol{F}_{k} E\left[\boldsymbol{e}_{k-1} \mid \boldsymbol{Y}_{k-1}\right]
$$


where $E\left[\boldsymbol{e}_{k-1} \mid \boldsymbol{Y}_{k-1}\right]=0$. The predicted value of $\hat{\boldsymbol{x}}_{k \mid k-1}$ is then given by:

$$
\hat{\boldsymbol{x}}_{k \mid k-1} \approx f\left(\hat{\boldsymbol{x}}_{k-1 \mid k-1}, \boldsymbol{u}_{k}\right)
$$

The prediction error equation is defined as:

$$
\boldsymbol{e}_{k \mid k-1}=\boldsymbol{x}_{k}-\hat{\boldsymbol{x}}_{k \mid k-1} \approx \boldsymbol{F}_{k} \boldsymbol{e}_{k-1}+\boldsymbol{\omega}_{k-1}
$$

Thus, the prediction error covariance is given by:

$$
\begin{gathered}
\boldsymbol{P}_{k \mid k-1}=E\left[\boldsymbol{e}_{k \mid k-1} \boldsymbol{e}_{k \mid k-1}^{T}\right] \\
\boldsymbol{P}_{k \mid k-1}=\boldsymbol{F}_{k} E\left[\boldsymbol{e}_{k-1} \boldsymbol{e}_{k-1}^{T}\right] \boldsymbol{F}_{k}^{T}+E\left[\boldsymbol{\omega}_{k-1} \boldsymbol{\omega}_{k-1}^{T}\right] \\
\boldsymbol{P}_{k \mid k-1}=\boldsymbol{F}_{k} \boldsymbol{P}_{k-1 \mid k-1} \boldsymbol{F}_{k}^{T}+\boldsymbol{Q}_{k-1}
\end{gathered}
$$

\section{Update}

At time $k$ we have the measurement $\boldsymbol{y}_{k}$ with the covariance $\boldsymbol{R}_{k}$, and EKF is approximating the best unbiased estimate as:

$$
\hat{\boldsymbol{x}}_{k \mid k}=\hat{\boldsymbol{x}}_{k \mid k-1}+\boldsymbol{K}_{k}\left(\boldsymbol{y}_{k}-\boldsymbol{h}\left(\hat{\boldsymbol{x}}_{k \mid k-1}\right)\right)
$$

The covariance matrix $\boldsymbol{P}_{k \mid k}$ is defined as:

$$
\begin{gathered}
\boldsymbol{P}_{k \mid k}=\operatorname{cov}\left(\boldsymbol{x}_{k}-\hat{\boldsymbol{x}}_{k \mid k}\right) \\
\boldsymbol{P}_{k \mid k}=\operatorname{cov}\left(\boldsymbol{x}_{k}-\left[\hat{\boldsymbol{x}}_{k \mid k-1}+\boldsymbol{K}_{k}\left(\boldsymbol{y}_{k}-\boldsymbol{H}_{k} \hat{\boldsymbol{x}}_{k \mid k-1}\right)\right]\right)
\end{gathered}
$$


where

$$
\left.\boldsymbol{H}_{k} \approx \frac{\partial \boldsymbol{h}}{\partial \boldsymbol{x}}\right|_{\hat{\boldsymbol{x}}_{k \mid k}}
$$

By the properties of vector covariance, the Equation (2.126) can be rewritten as:

$$
\boldsymbol{P}_{k \mid k}=\left(\boldsymbol{I}-\boldsymbol{K}_{k} \boldsymbol{H}_{k}\right) \operatorname{cov}\left(\boldsymbol{x}_{k}-\hat{\boldsymbol{x}}_{k \mid k-1}\right)\left(\boldsymbol{I}-\boldsymbol{K}_{k} \boldsymbol{H}_{k}\right)^{T}+\boldsymbol{K}_{k} \operatorname{cov}\left(\boldsymbol{v}_{k}\right) \boldsymbol{K}_{k}^{T}
$$

Using an invariant on $P_{k \mid k-1}$ and the definition of $R_{k}, \boldsymbol{P}_{k \mid k}$ is given by:

$$
\begin{gathered}
\boldsymbol{P}_{k \mid k}=\boldsymbol{P}_{k \mid k-1}-\boldsymbol{K}_{k} \boldsymbol{H}_{k} \boldsymbol{P}_{k \mid k-1}-\boldsymbol{P}_{k \mid k-1} \boldsymbol{H}_{k}^{T} \boldsymbol{K}_{k}^{T}+\boldsymbol{K}_{k}\left(\boldsymbol{H}_{k} \boldsymbol{P}_{k \mid k-1} \boldsymbol{H}_{k}^{T}+\boldsymbol{R}_{k}\right) \boldsymbol{K}_{k}^{\boldsymbol{T}} \\
=\boldsymbol{P}_{k \mid k-1}-\boldsymbol{K}_{k} \boldsymbol{H}_{k} \boldsymbol{P}_{k \mid k-1}-\boldsymbol{P}_{k \mid k-1} \boldsymbol{H}_{k}^{T} \boldsymbol{K}_{k}^{T}+\boldsymbol{K}_{k} \boldsymbol{S}_{k} \boldsymbol{K}_{k}^{\boldsymbol{T}}
\end{gathered}
$$

The last two terms cancel each other out, which can be simplified further as shown below:

$$
\begin{gathered}
\boldsymbol{K}_{k} \boldsymbol{S}_{k} \boldsymbol{K}_{k}^{\boldsymbol{T}}=\boldsymbol{P}_{k \mid k-1} \boldsymbol{H}_{k}^{T} \boldsymbol{K}_{k}^{T} \\
\boldsymbol{P}_{k \mid k}=\boldsymbol{P}_{k \mid k-1}-\boldsymbol{K}_{k} \boldsymbol{H}_{k} \boldsymbol{P}_{k \mid k-1}=\left(\boldsymbol{I}-\boldsymbol{K}_{k} \boldsymbol{H}_{k}\right) \boldsymbol{P}_{k \mid k-1}
\end{gathered}
$$

where $K_{k}$ is the optimal Kalman gain. We want to minimize the expected value of the square of the vector magnitude $E\left[\left\|\boldsymbol{x}_{k}-\hat{\boldsymbol{x}}_{k \mid k}\right\|^{2}\right]$. This is equivalent to minimizing the trace of the posteriori estimate covariance matrix $\boldsymbol{P}_{k \mid k}$. The trace is minimized when its matrix derivative with respect to the gain matrix is zero, so we have:

$$
\frac{\partial \operatorname{trace}\left(\boldsymbol{P}_{k \mid k}\right)}{\partial \boldsymbol{K}_{k}}=-2\left(\boldsymbol{H}_{k} \boldsymbol{P}_{k \mid k-1}\right)^{T}+2 \boldsymbol{K}_{k} \boldsymbol{S}_{k}=0
$$

Solving this for $\boldsymbol{K}_{k}$ gives the Kalman gain as:

$$
\boldsymbol{K}_{k}=\boldsymbol{P}_{k \mid k-1} \boldsymbol{H}_{k}^{T}\left(\boldsymbol{H}_{k} \boldsymbol{P}_{k \mid k-1} \boldsymbol{H}_{k}^{T}+\boldsymbol{R}\right)^{-1}
$$




\section{Summary of the Extended Kalman Filter}

The equations for EKF are defined by the following recursive equations [128].

\section{Initialization}

$$
\hat{\boldsymbol{x}}_{0 \mid 0}=E\left(\boldsymbol{x}_{0}\right), \quad \boldsymbol{P}_{0 \mid 0}=E\left[\left(\boldsymbol{x}_{0}-\hat{\boldsymbol{x}}_{0 \mid 0}\right)\left(\boldsymbol{x}_{0}-\hat{\boldsymbol{x}}_{0 \mid 0}\right)^{T}\right]
$$

\section{Prediction}

$$
\begin{gathered}
\hat{\boldsymbol{x}}_{k \mid k-1}=f\left(\hat{\boldsymbol{x}}_{k-1 \mid k-1}, \boldsymbol{u}_{k}\right) \\
\boldsymbol{P}_{k \mid k-1}=\boldsymbol{F}_{k} \boldsymbol{P}_{k-1 \mid k-1} \boldsymbol{F}_{k}^{T}+\boldsymbol{Q}_{k}
\end{gathered}
$$

\section{Update}

$$
\begin{gathered}
\boldsymbol{K}_{k}=\boldsymbol{P}_{k \mid k-1} \boldsymbol{H}_{k}^{T}\left(\boldsymbol{H}_{k} \boldsymbol{P}_{k \mid k-1} \boldsymbol{H}_{k}^{T}+\boldsymbol{R}\right)^{-1} \\
\hat{\boldsymbol{x}}_{k \mid k}=\hat{\boldsymbol{x}}_{k \mid k-1}+\boldsymbol{K}_{k}\left(\boldsymbol{y}_{k}-\boldsymbol{h}\left(\hat{\boldsymbol{x}}_{k \mid k-1}\right)\right) \\
\boldsymbol{P}_{k \mid k}=\left(\boldsymbol{I}-\boldsymbol{K}_{k} \boldsymbol{H}_{k}\right) \boldsymbol{P}_{k \mid k-1}
\end{gathered}
$$

\subsubsection{Iterated Extended Kalman Filter}

The model and implementation equations for the weighted Iterated Extended Kalman

Filter are defined as the following recursive equations [129]: 
Initialization

$$
\hat{\boldsymbol{x}}_{0 \mid 0}=E\left(\boldsymbol{x}_{0}\right), \quad \boldsymbol{P}_{0 \mid 0}=E\left[\left(\boldsymbol{x}_{0}-\hat{\boldsymbol{x}}_{0 \mid 0}\right)\left(\boldsymbol{x}_{0}-\hat{\boldsymbol{x}}_{0 \mid 0}\right)^{T}\right]
$$

\section{Prediction}

The predicted state can be defined as:

$$
\begin{gathered}
\hat{\boldsymbol{x}}_{k \mid k-1}=\boldsymbol{f}\left(\hat{\mathbf{x}}_{k-1 \mid k-1}, \boldsymbol{u}_{k}\right) \\
\boldsymbol{P}_{k \mid k-1}=\boldsymbol{F}_{k} \boldsymbol{P}_{k-1 \mid k-1} \boldsymbol{F}_{k}^{T}+\boldsymbol{Q}_{k-1}
\end{gathered}
$$

\section{Update}

The Kalman gain can be computed as:

$$
\mathbf{K}_{k, i}=\mathbf{P}_{k \mid k-1} \mathbf{H}_{k, i}^{T}\left(\mathbf{H}_{k, i} \mathbf{P}_{k \mid k-1} \mathbf{H}_{k, i}^{T}+\mathbf{R}_{k}\right)^{-1}
$$

where

$$
\left.\boldsymbol{H}_{k, i} \approx \frac{\partial \boldsymbol{h}}{\partial \boldsymbol{x}}\right|_{\hat{\boldsymbol{x}}_{k \mid k, i}}
$$

The superscript $i,(i=0,2, \ldots, \tau)$, is the number of iteration steps. The predicted measurement estimation can be rewritten as:

$$
\hat{\boldsymbol{x}}_{k \mid k, i+1}=\hat{\boldsymbol{x}}_{k \mid k-1}+\boldsymbol{K}_{k, i}\left(\boldsymbol{y}_{k}-\boldsymbol{h}\left(\hat{\boldsymbol{x}}_{k \mid k, i}\right)-\boldsymbol{H}_{k, i}\left(\hat{\boldsymbol{x}}_{k \mid k-1}-\hat{\boldsymbol{x}}_{k \mid k, i}\right)\right)
$$

where, $\hat{\boldsymbol{x}}_{k \mid k, i+1}$ is the estimate at time point $k$. The posterior covariance matrix is 
defined as:

$$
\boldsymbol{P}_{k \mid k, i}=\left(\boldsymbol{I}-\boldsymbol{K}_{k, i} \boldsymbol{H}_{k, i}\right) \boldsymbol{P}_{k \mid k-1}
$$

\subsubsection{Unscented Kalman Filter}

\section{Initialization}

$$
\hat{\boldsymbol{x}}_{0 \mid 0}=E\left(\boldsymbol{x}_{0}\right), \quad \boldsymbol{P}_{0 \mid 0}=E\left[\left(\boldsymbol{x}_{0}-\hat{\boldsymbol{x}}_{0 \mid 0}\right)\left(\boldsymbol{x}_{0}-\hat{\boldsymbol{x}}_{0 \mid 0}\right)^{T}\right]
$$

\section{Calculate sigma points}

For the $L$ elements state vector, a set of $(2 L+1)$ sigma-points are created according to the following [130]:

$$
\chi_{k-1}=\left[\begin{array}{lll}
\hat{\boldsymbol{x}}_{k-1} & \hat{\boldsymbol{x}}_{k-1}+\sqrt{(L+\lambda)} \sqrt{\boldsymbol{P}_{k-1}} & \hat{\boldsymbol{x}}_{k-1}-\sqrt{(L+\lambda)} \sqrt{\mathbf{P}_{k-1}}
\end{array}\right]
$$

where each column of $\chi_{k-1}$, represents a sigma-point. $\sqrt{\mathbf{P}_{k-1}}=\operatorname{chol}\left(\mathbf{P}_{k-1}\right)$ is the square root of the state error covariance, and the scaling parameter $\lambda$ defined as:

$$
\lambda=\sigma^{2}(L+\kappa)-L
$$

where $e^{-4} \leq \sigma \leq 1$ determines the size of the sigma-points distribution, and $\kappa$ influences the accuracy of the approximation. 


\section{Prediction}

Once the sigma-points have been generated, each point is propagated throughout the non-linear state equation as:

$$
\chi_{k \mid k-1}^{(i)}=\mathbf{f}\left(\boldsymbol{\chi}_{k-1}, \mathbf{u}_{k}\right), \quad i=0, \ldots, 2 L
$$

The mean and covariance are approximated using a weighted mean, and the covariance of the transformed points is:

$$
\begin{gathered}
\hat{\mathbf{x}}_{k \mid k-1}=\sum_{i=0}^{2 L} \boldsymbol{\eta}_{i}^{(m)} \boldsymbol{\chi}_{k \mid k-1}^{(i)} \\
\mathbf{P}_{k \mid k-1}=\sum_{i=0}^{2 L} \boldsymbol{\eta}_{i}^{(c)}\left(\boldsymbol{\chi}_{k \mid k-1}^{(i)}-\hat{\boldsymbol{x}}_{k \mid k-1}\right)\left(\boldsymbol{\chi}_{k \mid k-1}^{(i)}-\hat{\boldsymbol{x}}_{k \mid k-1}\right)^{T}+\boldsymbol{Q}_{k}
\end{gathered}
$$

where the mean weight vector $\boldsymbol{\eta}_{i}^{(m)}$ and the covariance weight vector $\boldsymbol{\eta}_{i}^{(c)}$ associated with the $i_{t h}$ point are defined as:

$$
\begin{gathered}
\boldsymbol{\eta}_{i}^{(c)}=\boldsymbol{\eta}_{i}^{(m)}=\frac{1}{2(L+\lambda)}, \quad i=1, \ldots, 2 L \\
\gamma_{k \mid k-1}^{(i)}=\mathbf{h}\left(\boldsymbol{\chi}_{k \mid k-1}^{(i)}\right)
\end{gathered}
$$

Then the mean of the measurement vector is calculated as:

$$
\hat{\boldsymbol{y}}_{k \mid k-1}=\sum_{i=0}^{2 L} \boldsymbol{\eta}_{i}^{(m)} \boldsymbol{\gamma}_{k \mid k-1}^{(i)}
$$




\section{Update}

Covariance and cross-covariance matrices of the UKF are defined as:

$$
\begin{gathered}
\boldsymbol{P}_{k}^{y y}=\sum_{i=0}^{2 L} \eta_{i}^{(c)}\left(\boldsymbol{\gamma}_{k \mid k-1}^{(i)}-\hat{\boldsymbol{y}}_{k \mid k-1}\right)\left(\boldsymbol{\gamma}_{k \mid k-1}^{(i)}-\hat{\boldsymbol{y}}_{k \mid k-1}\right)^{T}+\boldsymbol{R}_{k} \\
\boldsymbol{P}_{k}^{x y}=\sum_{i=0}^{2 L} \eta_{i}^{(c)}\left(\boldsymbol{\chi}_{k \mid k-1}^{(i)}-\hat{\boldsymbol{x}}_{k \mid k-1}\right)\left(\boldsymbol{\gamma}_{k \mid k-1}^{(i)}-\hat{\boldsymbol{y}}_{k \mid k-1}\right)^{T} \\
\boldsymbol{K}_{k}=\boldsymbol{P}_{k}^{x y}\left(\boldsymbol{P}_{k}^{y y}\right)^{-1}
\end{gathered}
$$

The Kalman gain $\boldsymbol{K}_{k}$ is then used to update the state and covariance estimates as:

$$
\begin{gathered}
\hat{\boldsymbol{x}}_{k \mid k}=\hat{\boldsymbol{x}}_{k \mid k-1}+\boldsymbol{K}_{k}\left(\boldsymbol{y}_{k}-\hat{\boldsymbol{y}}_{k \mid k-1}\right) \\
\boldsymbol{P}_{k \mid k}=\boldsymbol{P}_{k \mid k-1}-\boldsymbol{K}_{k} \boldsymbol{P}_{k}^{y y} \boldsymbol{K}_{k}^{T}
\end{gathered}
$$

\subsection{Fuzzy Logic}

A Fuzzy Logic System (FLS) is defined as the non-linear mapping of an input data set to scalar output data [131]. It consists of four main elements: the fuzzifier, rules, inference and a defuzzifier. The general architecture of an FLS is shown in Figure. 2.5. Firstly, input data are gathered and converted to fuzzy sets using fuzzy linguistic variables, terms and membership functions. This step is known as fuzzification. Subsequently, an inference is made based on a set of rules. Finally, the resulting fuzzy output is mapped to output using the membership functions in the defuzzification step. 


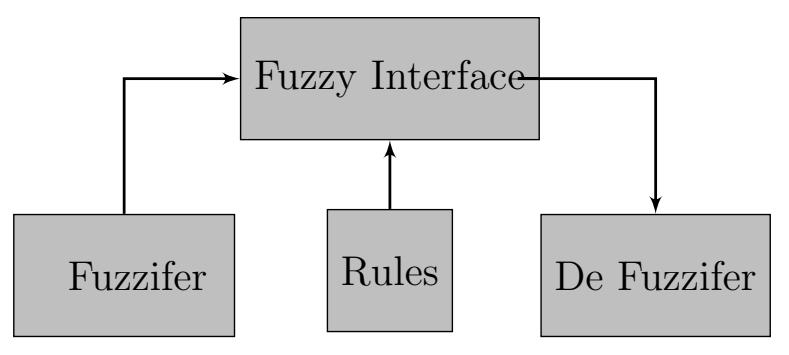

Figure. 2.5: Fuzzy Logic Controller.

\subsubsection{Membership Function}

To map non-fuzzy input values to fuzzy linguistic terms, and vice versa, membership functions are used in the fuzzification and defuzzification steps. There are various forms of membership functions, including trapezoidal, triangular, Gaussian and piecewise linear. Triangular, trapezoidal, and Gaussian shapes are most commonly used for membership functions. The type of the membership function is generally chosen according to the user experience.

\subsubsection{Fuzzy Rules}

In an FLS, rules are applied to control the output variable. A fuzzy rule is a simple IF-THEN type, with a condition and a conclusion [132]. The evaluations of the fuzzy rules and the combination of the results of the individual rules are performed using fuzzy set operations. The operations on fuzzy sets are different than the operations on non-fuzzy sets.

\section{Fuzzy set operations}

The evaluations of the fuzzy rules, the combination of the results of the individual rules are performed using fuzzy set operations [133]. Table 2.1 indicates possible fuzzy functions for AND and OR operators on these sets. $\mu_{A}$ and $\mu_{B}$ are the membership functions for fuzzy sets $A$ and $B$. Notably, the most used operations for AND and 
OR operators are max and min, respectively.

Table 2.1: Fuzzy operations for AND and OR operators.

\begin{tabular}{lccc}
\hline & OR & AND \\
\hline \hline $\operatorname{Max}$ & $\operatorname{Max}\left(\mu_{A}(x)+\mu_{B}(x)\right)$ & Min & $\operatorname{Min}\left(\mu_{A}(x), \mu_{B}(x)\right)$ \\
ASUM & $\mu_{A}(x)+\mu_{B}(x)-\mu_{A}(x) \mu_{B}(x)$ & PROD & $\mu_{A}(x) \mu_{B}(x)$ \\
BSUM & $\operatorname{Max}\left(1, \mu_{A}(x)+\mu_{B}(x)\right)$ & BDIF & $\operatorname{Max}\left(0, \mu_{A}(x)+\mu_{B}(x)-1\right)$ \\
\hline
\end{tabular}

In order to determine the final result, the results of each rule must be combined, a process known as the interface. The rules can be combined in various ways, and Table 2.2 summarizes possible methods to achieve the combinations. The Maximum algorithm is typically used for accumulation.

Table 2.2: Possible Fuzzy rules combination.

\begin{tabular}{lc}
\hline Operation & Formula \\
\hline \hline Maximum & $\operatorname{Max}\left(\mu_{A}(x), \mu_{B}(x)\right)$ \\
Bounded Sum & $\operatorname{Min}\left(1, \mu_{A}(x)+\mu_{B}(x)\right)$ \\
Normalized Sum & $\frac{\mu_{A}(x)+\mu_{B}(x)}{\operatorname{Max}\left(1, \mu_{A}(x)+\mu_{B}(x)\right)}$ \\
\hline
\end{tabular}

\subsubsection{Defuzzification}

After the inference step, to obtain the final output, the fuzzy value should be defuzzified. Defuzzification is performed according to the membership function of the output variable. Different techniques were used for the defuzzification step [134]. The most well-known algorithms are listed in Table 2.3. 
Table 2.3: The most well-known defuzzification methods.

\begin{tabular}{lc}
\hline \hline Operation & Formula \\
\hline Centroid & $U=\frac{\int_{\min }^{\max } u \mu(u) d u}{\int_{\min }^{\max } \mu(u) d u}$ \\
Center of Gravity for Singletons & $U=\frac{\sum_{i=1}^{m}\left[u_{i} \mu_{i}\right]}{\sum_{i=1}^{p} \mu_{i}}$ \\
Left Most Maximum & $U=\inf (u), \mu(u)=\sup (\mu(u))$ \\
Right Most Maximum & $U=\sup (u), \mu(u)=\sup (\mu(u))$ \\
\hline \hline
\end{tabular}




\section{Chapter 3}

\section{Uncooperative Space Target Detection and Tracking}

This section develops a new feature-based tracking algorithm specifically for uncooperative space targets. The objective is to design a novel tracking algorithm that is robust and accurate under a variety of environmental conditions and motions. This study proposes an adaptive Unscented Kalman filter, which can be employed to autonomously locate and track unknown space targets to enhance orbital target tracking capabilities. Specifically, to detect high uncertainty associate with miss tracking and protect the UKF from divergence, a fuzzy logic adaptation mechanism is designed to apply an appropriate weighting factor to tune the $\boldsymbol{Q}_{k}$ and $\boldsymbol{R}_{k}$ matrices. Experimental results indicate that the suggested approach can significantly improve target-tracking performance and provide good tracking capability. Performance comparisons with the proposed FAUKF, UKF, and EKF were also conducted. Notably, due to the minimal processing time required, the suggested framework can track targets in real-time. 


\subsection{Introduction}

Due to human activities in recent decades, space is now congested with many objects, including satellites and space debris. Many current and future orbital operations involve automated physical interactions with free-floating and free-flying targets. A full navigation solution must have precise knowledge of a target's pose and motion. However, this information is typically unavailable when damaged satellites, space debris, and vibrating structures are involved. For target pose and motion estimation, realtime tracking capabilities are a core component of many formation flights, including servicing satellites, autonomous rendezvous and docking, inspection, space debris removal, and robotic maintenance [135]. Though the requirements for each application vary, robust, accurate, efficient and fast visual tracking under a variety of conditions, such as different illumination, fast motions, full or partial object occlusion and lack of prior information about a target is critical. Furthermore, in some cases, the target of interest does not have a rigid body, so the size and appearance can change over time. All these conditions make target detection and tracking highly challenging [136]. Feature detection is the initial step of visual tracking, and due to the high dimensionality of image data, tracking every pixel is computationally prohibitive. Instead, a sparse set of features is extracted from the image, such as corners, blobs and edges. The visual feature detection techniques can be classified into three main categories: edge detection, corner detection, and blob detection. The edge refers to abrupt changes in pixel brightness. The two key methods of edge detectors are differential-based and learning-based. Well known differential-based edge detectors include Sobel [137], Roberts-cross, Laplacian of Gaussian (LOG) [138], Oriented Energy (OE), Canny edge detector [139], Color boundary [140]. Soble and Roberts-cross are the first-order 
differential-based edge detection operators that compute the gradient at various orientations, and LOG is the second-order differentiation. OE is based on directional differentiation, and to detect changes in pixel brightness, a batch of filters are applied at different orientations. Though differential-based edge detection is relatively simple, sensitivity to noise is a shortcoming of this method. Concerning learning-based methods, edge detection is modeled as a machine learning-based framework that distinguishes smooth regions from edges. The Probability-of-boundary (Pb) [141], Boosted Edge Learning (BEL) [142], Multi-Scale Pb (MS-Pb) [143], texture-based $\mathrm{Pb}(\mathrm{tPb})$ [144], glocal PB (gPb) [145], Sparse Code Gradient (SCG) detection [146] and Sketch Tokens are among the well-known differential-based edge detectors. The intersecting point of two connected straight edges is defined as a corner, and corner detection methods are gradient-based, template-based, and contour-based. Gradientbased corner detection is based on gradient calculation, and early corner detection algorithms are mostly based on this method. The Harris [147], Shi-Tomasi [148] and LOCOCO [149] are examples of gradient-based corner detectors. Template-based corner detection is based on comparisons of pixels, with corners being detected by comparing the intensity of the surrounding pixels with the center pixel. SUSAN [150] and FAST [151] are also gradient-based corner detectors. Blob detectors are classified as interest point detection and interest region-based detection. Interest point detection finds local extremes in pyramid spaces. The Difference of Gaussian (DoG), DoH, Hessian-Laplacian [152], SIFT [153], SURF [154], Cer-SURF [155], RLOG [156], MOGP [157], KAZE [158], ORB [159], BRISK [160] and FREAK [161] are among the well-known Blob detectors. Interest region detection aims to identify regions with constant segmentation techniques and includes interest region segmentation-based MSER [162], IBR [163], Salient region [164], PCBR [165], Beta-stable feature [166], MFD [167] and MSCR [168]. After object detection, tracking the target object is 
required. Object tracking technologies have been studied and improved for several years and can be divided into four main categories: model-based [169-171], regionbased [172-174], contour-based [175-177], and feature-based [178-181]. Model-based target tracking requires prior knowledge of the object; by matching the model to the image data, this method can recognize and locate the object [182]. However, access to a detailed model of the space target is unlikely, particularly if the space targets are uncooperative. This class of methods is computationally expensive [183]. Region-based tracking is another approach employed for object tracking [184]. The objective of region-based tracking is to classify the target regions with histograms or other non-parametric descriptions and then track them over time. However, in the case of partial occlusion, segmenting the object is very difficult. In addition, this method cannot cope with a cluttered background. [183]. Contour-based object tracking tracks the contours of a moving object. Though this method is considered dual to the region-based tracking, it provides a more efficient description of the target than contour-based tracking. It can handle a diverse variety of objects since it depends on motion information to prepare the contour. It has high computational complexity and cannot handle large non-rigid movements and partial occlusion efficiently [183]. Feature-based tracking is another approach to target tracking. It has the advantage of coping with partial occlusion since some feature points could remain visible during occlusion. Selecting suitable features is important, and features used for tracking need to be unique so the target can be distinctive. Once feature points that represent the target have been detected, they can be tracked over video sequences. A detailed description of such target tracking methods can be found in [185]. The classic approach for tracking features of a target object is a probabilistic estimation, as found in frameworks such as KF [186]. However, the Kalman filter is only applicable when the dynamic and measurement processes are known and liner. 
To address this constraint, other types of Kalman filters were designed for nonlinear systems, including the Extended Kalman filter [187], the Unscented Kalman filter, the Central-Difference Kalman filter [188] and the structural Kalman filter. However, due to the non-linearity and non-Gaussianity associated with orbital measurements and dynamics, tracking a space object with regular filters is very challenging $[189,190]$. In such cases, particle filters and adaptive filtering techniques can be applied. The particle filter in this case, is a robust nonlinear Monte-Carlo estimator [191, 192], with an inherently high computational load. On the other hand, adaptive Kalman filters accomplish a good compromise between computational load and performance. An adaptive Kalman filter for human tracking was proposed by Weng et al. [193]. In this framework, the occlusion rate was employed to adjust the covariance error. Orbital target tracking with an adaptive filter was suggested in [194]. In their work, only the covariance matrix $\boldsymbol{Q}_{k}$ was adaptively tuned, employing the current state and covariance estimate in a gravity-gradient model.

\subsection{Testbeds}

To evaluate the proposed approaches throughout this thesis two testbeds that can accurately represent spinning motions and complex environmental conditions, including intense illumination, blueness and noise are used.

\subsubsection{Facility at the Spacecraft Robotics and Control Labo- ratory of Carleton University.}

The main testbed used in this study is shown in Figure. 3.1. It consists of 20,000 pounds, 8 feet by 12 feet gravity-offset air-bearing table, which emulates the dynamics of space and two air-bearing spacecraft platforms operating in close proximity on the 
air-bearing table surface. A reaction wheel actuates both platforms, and compressed air expelled through small air nozzles provides three degree-of-freedom control authority. The motion of both platforms is measured in real-time using LEDs that are tracked by an eight-camera motion capture system. This provides highly accurate ground truth. The visual sensor used is the ZED camera. Table 3.1 summarized the ZED camera specifications. To test the algorithms developed in this thesis under more realistic conditions, other footage was also used.

Table 3.1: ZED stereo camera specifications.

\begin{tabular}{cc}
\hline \hline Description & Specification \\
\hline Resolution & $64416 \times 1242,3840 \times 1080,2560 \times 720,1344 \times 376$ \\
Depth Range & $0.5-20 \mathrm{~m}(1.64$ to $65 \mathrm{ft})$ \\
Stereo Baseline & $120 \mathrm{~mm}\left(4.7^{\prime \prime}\right)$ \\
Field of View & $90^{\circ}(\mathrm{H}) \times 60^{\circ}(\mathrm{V}) \times 110^{\circ}(\mathrm{D}) \mathrm{max}$ \\
Dimensions & $175 \times 30 \times 33 \mathrm{~mm}\left(6.89^{\prime \prime} \times 1.18^{\prime \prime} \times 1.3^{\prime \prime}\right)$ \\
Power & $5 \mathrm{~V} / 380 \mathrm{~mA}$ \\
Weight & $159 \mathrm{~g}(0.35 \mathrm{lb})$ \\
\hline \hline
\end{tabular}




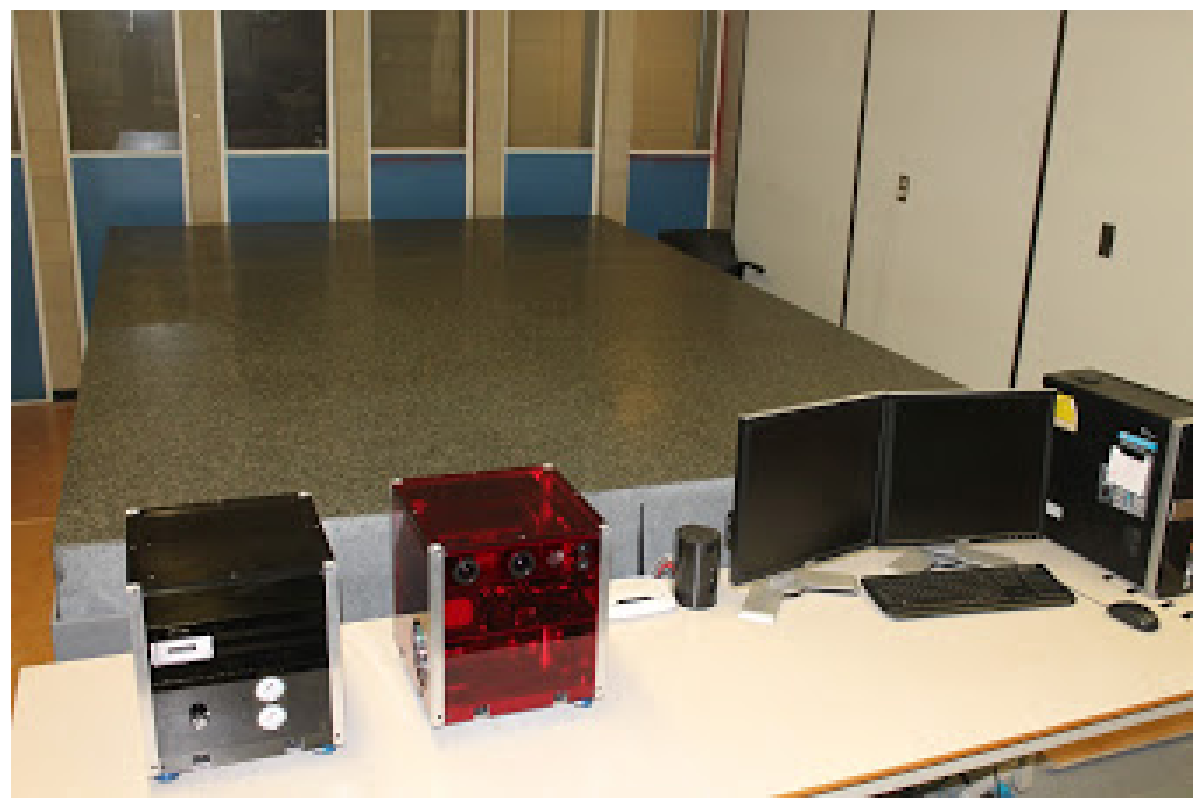

(a) Testbed.
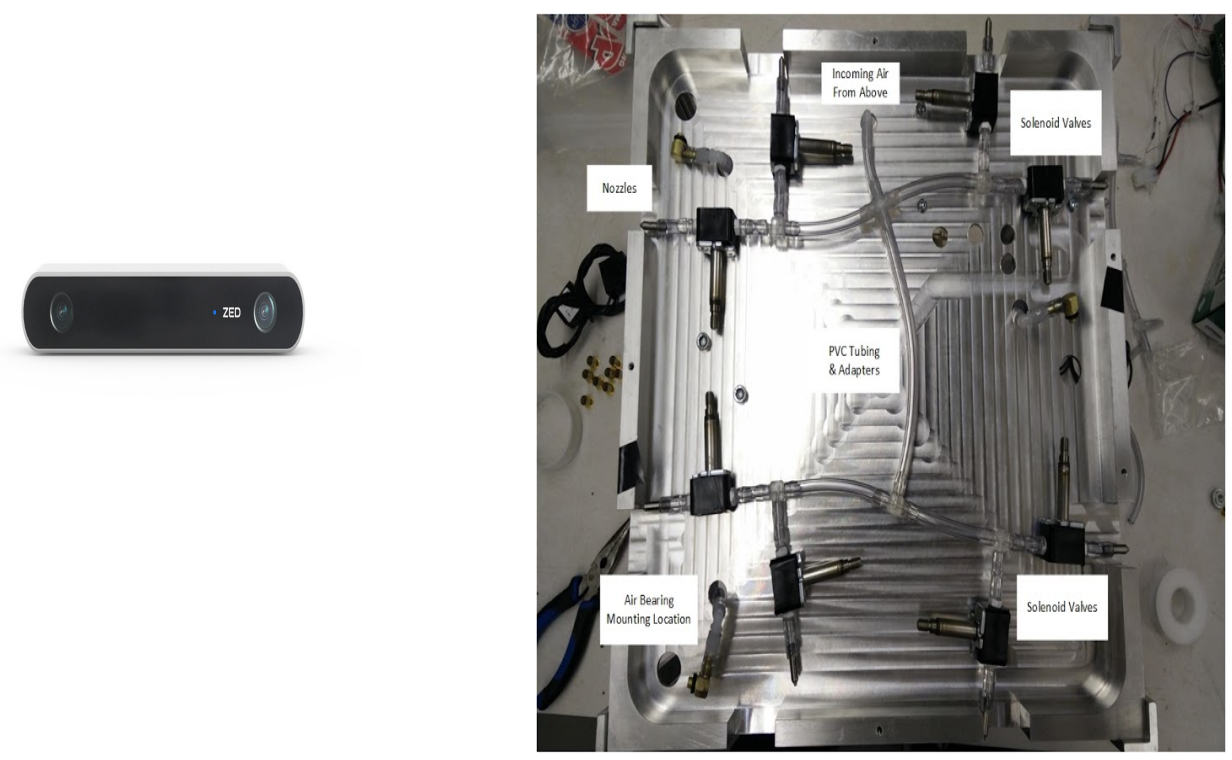

(b) ZED camera

(c) Air nozzles

Figure. 3.1: Testbed at the spacecraft robotics and control laboratory of Carleton University. 


\subsubsection{Actual TriDAR Images During STS-131 Space Shuttle Mission}

Actual images taken in orbit with Neptec's TriDAR thermal camera with a resolution of $640 \times 480$ pixels during the STS-131 space shuttle Discovery mission were used. For this mission, the TriDAR thermal camera was mounted on the space shuttle Discovery moving toward ISS, and it captured video sequences during docking, undocking, and fly-around of the ISS. A similar situation would be encountered during servicing operations of a tumbling non-cooperative space target [195]. The TriDAR thermal camera was specifically designed for non-cooperative missions such as satellite servicing, which require high levels of autonomy. It should be noted that the TriDAR camera can detect a target at a range of $43.4 \mathrm{~km}$ [195]. Throughout this mission, the video experienced diverse motion patterns, illumination conditions, blurriness and occlusion. The TriDAR camera is shown in Figure. 3.2, and a few frames from the footage are presented in Figure. 3.3. The TriDAR thermal camera specifications are summarized in Table 3.2.
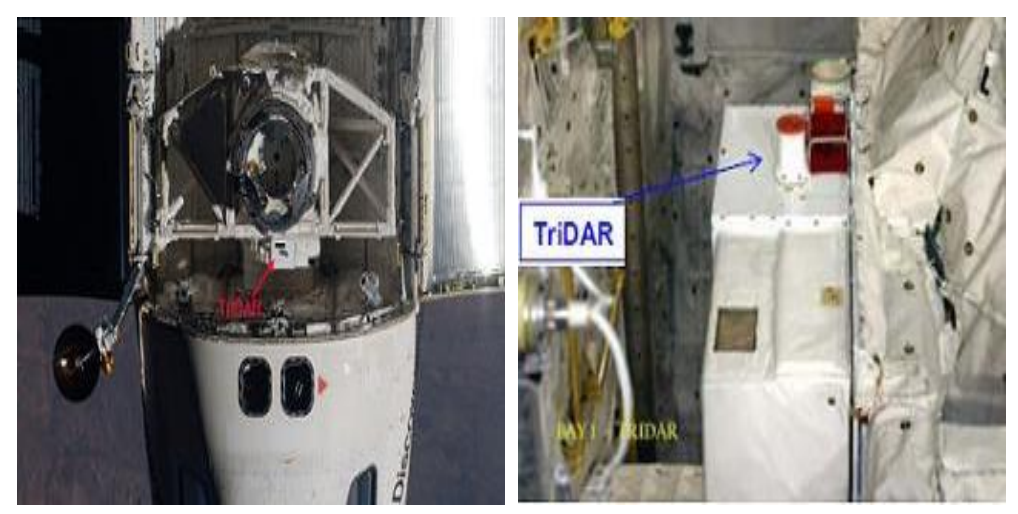

Figure. 3.2: TriDAR camera [4]. 
Table 3.2: TriDAR specifications.

\begin{tabular}{cc}
\hline \hline Description & Specification \\
\hline Flight Experience & Space Shuttle STS-128, STS-131 \\
Resolution & $640 \times 480$ \\
Bit Depth & 14 bits $/$ pixel \\
Infrared Band & $8-14 \mu \mathrm{m}$ \\
Operational Temperature & $-10^{\circ} \mathrm{C} \mathrm{to}+50^{\circ} \mathrm{C}$ \\
Dimensions & $105 \times 118 \times 93 \mathrm{~cm}$ \\
Max Power & $2 \mathrm{~W}$ \\
Nominal Power & $0.7 \mathrm{~W}$ \\
\hline \hline
\end{tabular}
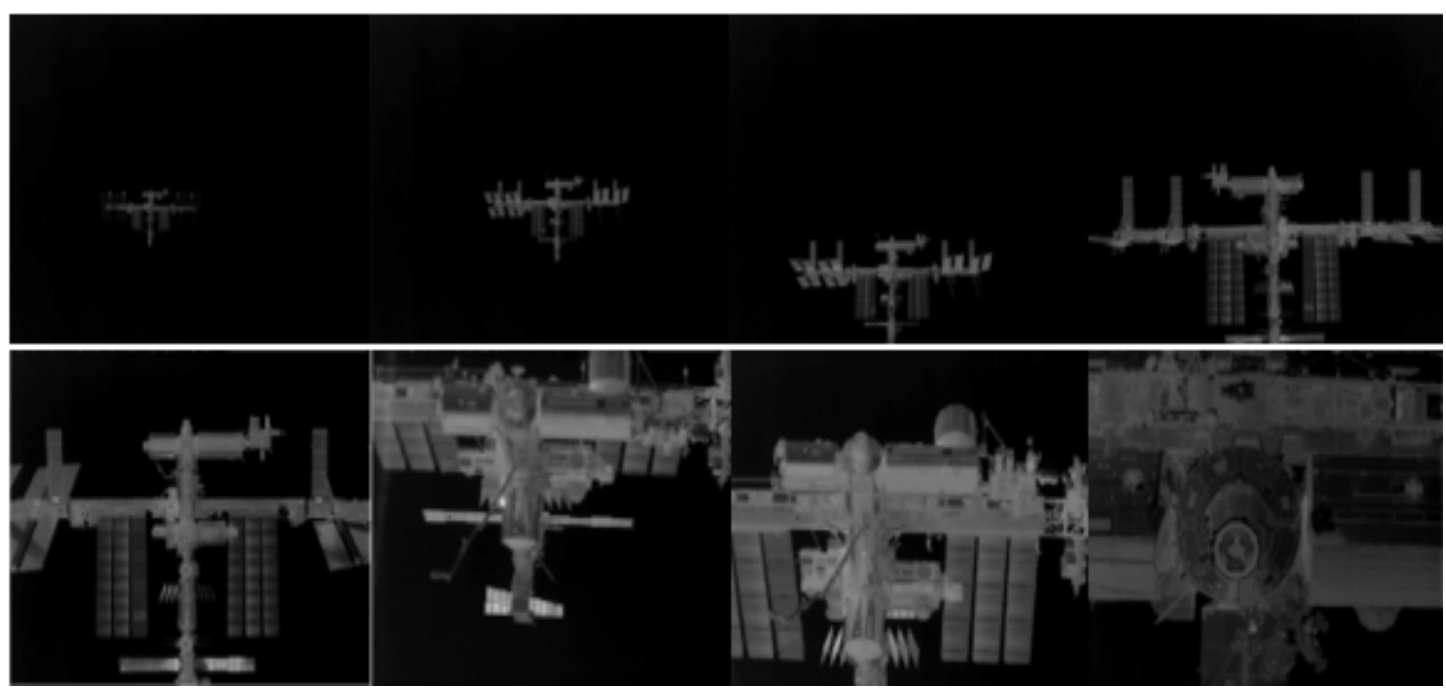

Figure. 3.3: Neptec's TriDAR video footage during a docking maneuver with the ISS (STS-131). 


\section{TriDAR coordinate system}

The TriDAR data coordinate system follows a standard 3-D OpenGL camera convention. As shown in Figure. 3.4, $X$ points to the right of the image plane, $Y$ points upward from the image plane and $Z$ points back from the viewpoint direction. The calibrated sensor focal point is at the center of the laser beam where it hits the $Y$ mirror (the sizeable vertical scanning mirror) [4]. The TriDAR field of view extends as a 30-degree squared cone from the focal point.

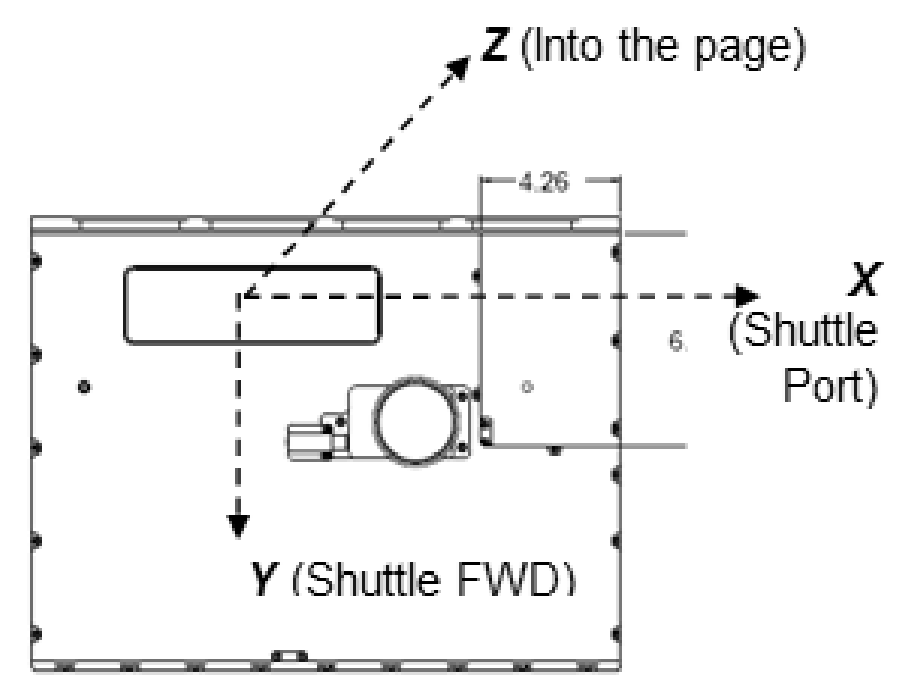

Figure. 3.4: TriDAR coordinate system.

\section{ISSACS coordinate system (ISSACS)}

The origin of the ISSACS is located at the geometric center of the Midship Truss Segment (ITS S0). The $X$ axis is parallel to the longitudinal axis of the US Lab Module and is directed toward Node 2, while the $Z$ axis points away from the radial port of the node. The $Y$ axis completes the right-handed coordinate system and corresponds to the alpha joint rotation axis (see Figure. 3.5). 


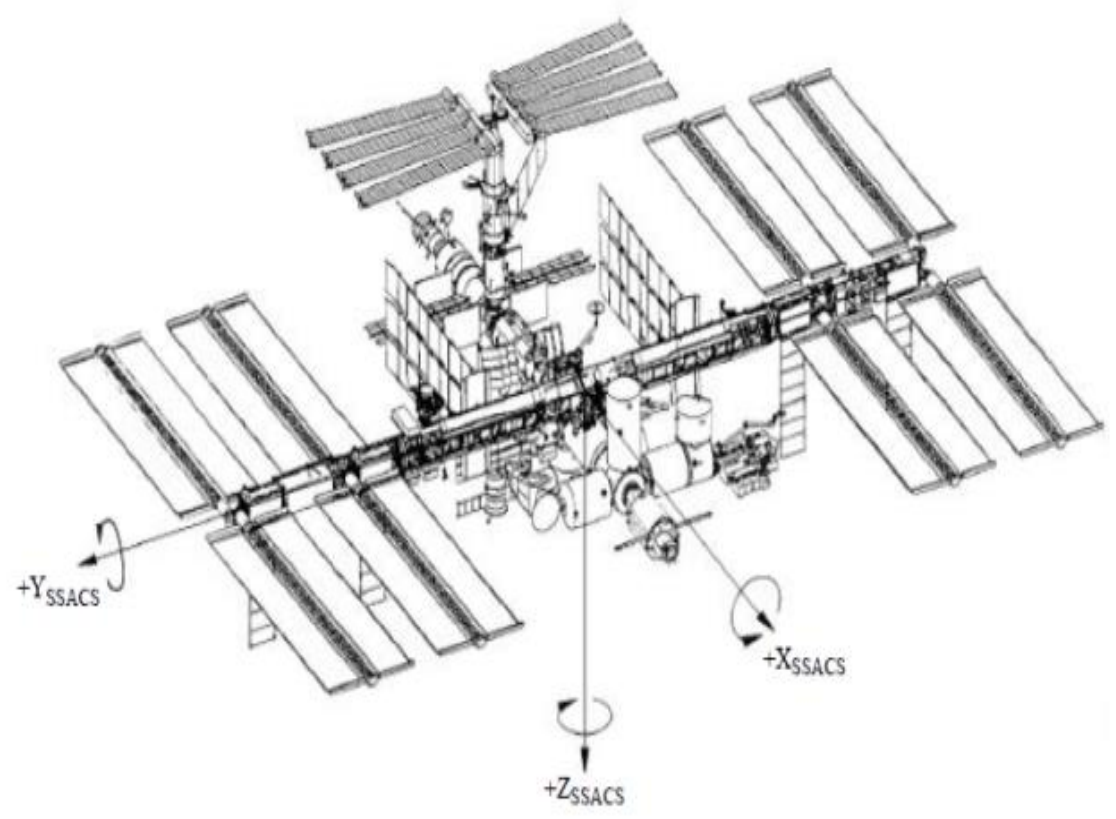

Figure. 3.5: ISS coordinate system [5].

\subsection{Target Detection}

The task of detecting interest points, feature descriptors, and matching between different images are the core components of all feature-based tracking. A variety of algorithms were proposed to accomplish these tasks, and comparison studies have also been conducted. However, to the best of our knowledge, there is no apparent work done to evaluate and compare these algorithms for real-time visual tracking of space applications under various conditions. Thus, this section aims to compare existing state-of-the-art algorithms and determine the best-perfumed feature detector, descriptor, matching, and outlier removal algorithms under various conditions that the visual system will encounter in space. Our evaluations use the above data set and include the following stages: (a) evaluation of the parameters of each algorithm to quantify their effect on detection performance and determine the optimal 
configuration for our task, (b) comparison of detectors under different conditions, (c) performance evaluation of feature matching and outlier removal method for a tracking solutions step, (d) generating disparity map using rectified images and triangulation.

\subsubsection{Background Subtraction}

One of the essential steps in vision-based applications is background subtraction. To recognize the target and track it over video frames, the background must be extracted. Background subtraction provides essential cues for numerous applications using computer vision. The OpenCV background subtraction was applied here. Figure. 3.6 shows the original image and the image after subtracting the background.

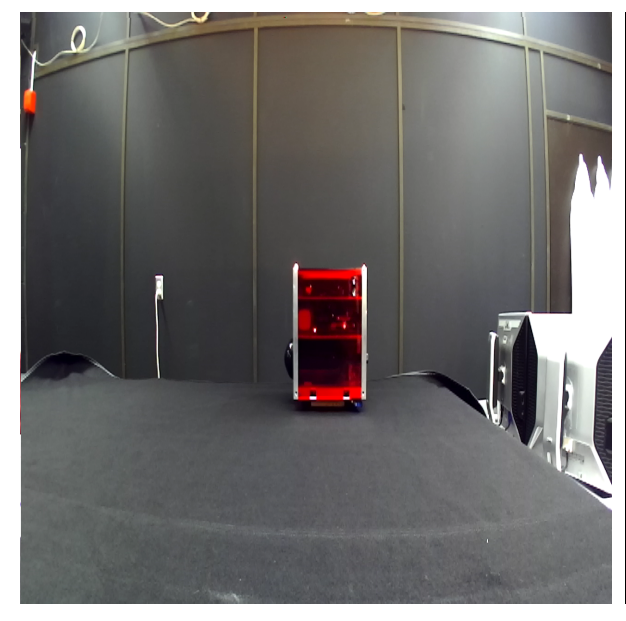

(a) Before subtraction

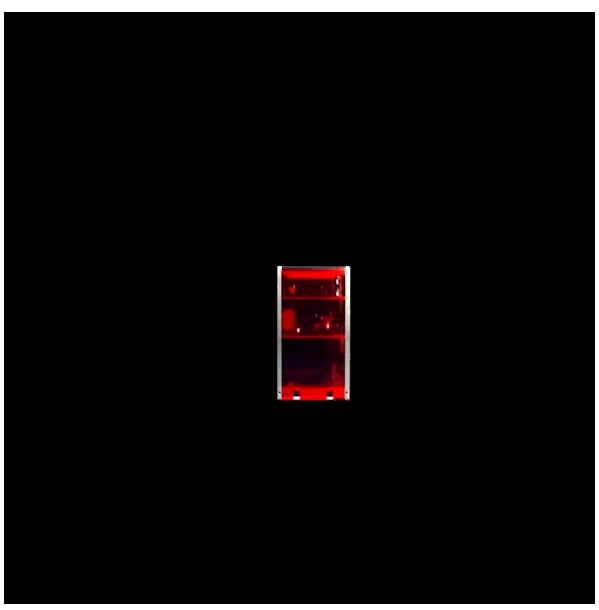

(b) After subtraction

Figure. 3.6: Background subtraction.

\subsubsection{Feature Detection}

The feature point detectors considered in this work are Harris, SURF, Fast, ShiTomasi, Brisk and MSER. These detectors were chosen since they have been used extensively and shown to outperform other detectors. The number of feature points 
is an important factor for feature point detector evaluation. Here, all selected interest point detectors are evaluated based on the number of feature points that the detector works well with under various conditions such as different illumination conditions, motions, distance, blurriness and noise level.

\section{Illumination}

High illumination is one of the main challenges in space, as it can impact featurebased tracking and reduce performance significantly. To evaluate the performance of implemented feature detectors, Images of the target were captured at three different illumination conditions: dark, light, and illuminated. These conditions were achieved by illuminating the target with reflectors with two intensities in a dark room. The comparison of six different feature point detectors based on the number of detected feature points shows in Figure. 3.7. As it shows, high illumination has the most significant impact on the number of the detected feature points.

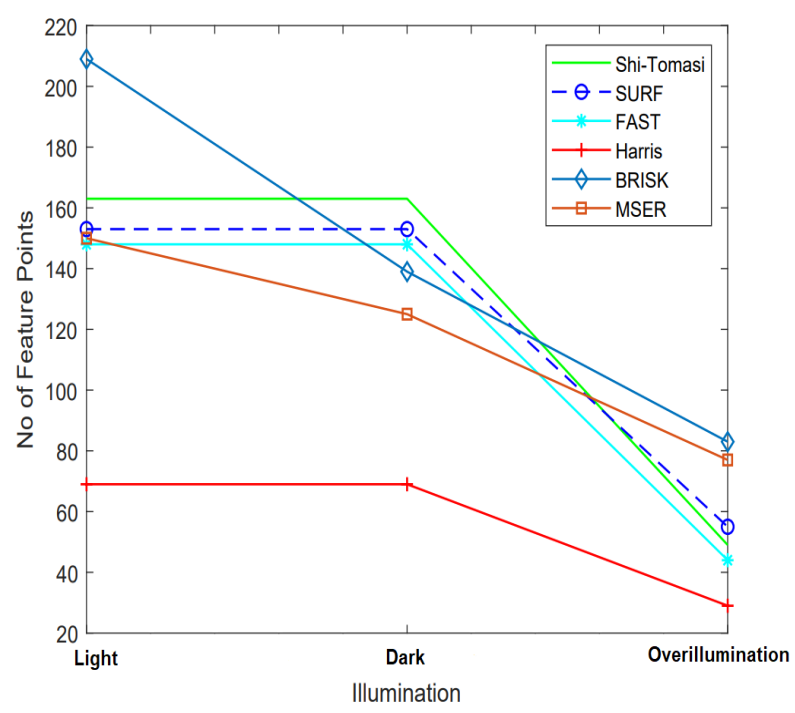

Figure. 3.7: Detectors' performance for different lightening conditions. 


\section{Tumbling motion}

Many space proximity operations, including on-orbit servicing, orbital debris removal, inspection, and robotic capture, involve a tumbling space target. Thus, the robustness and reliability of all relevant algorithms must be evaluated for performance with target motions. Figure. 3.8 shows the number of detected points achieved by different detectors at tumbling satellite rotation rates of $2.2,21.8$, and $2.2 \mathrm{deg} / \mathrm{s}$ about the roll, pitch and yaw axis, respectively. As it is illustrated, the SURF detector has the best performance in comparison with other detectors. In addition, different orientations have a significant effect on the number of detected feature points.

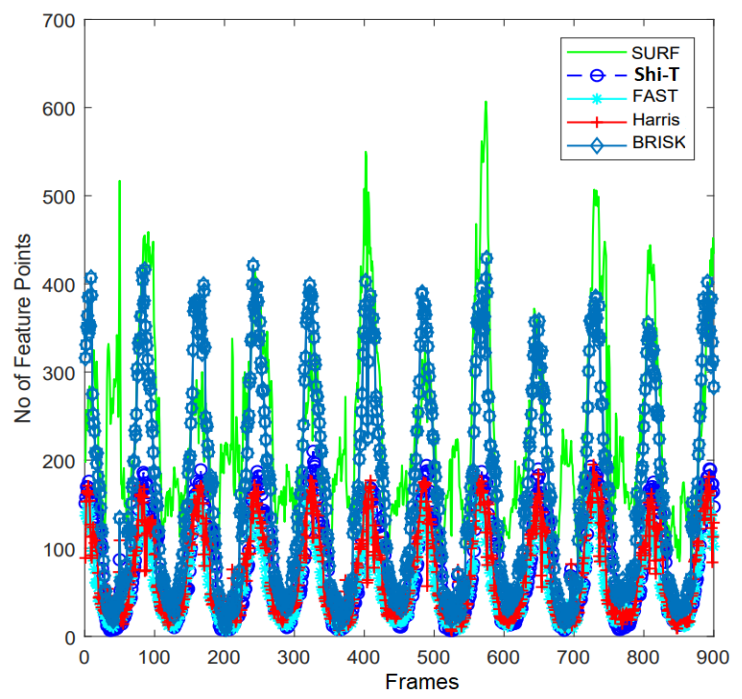

Figure. 3.8: Detectors' performance for tumbling motion of the space target.

\section{Motion blur}

The blurring of an image can be caused by many factors, such as movement during the image capture process, out-of-focus optics, and scattered light distortion. To evaluate the performance of the ditectors, five different levels of motion blur were added to the target spacecraft images, and the performance of all detectors was evaluated for motion blur levels. Figure. 3.9 shows the performance of the detectors. As shown, 
The motion blur reduces the number of detected features; thus, it negatively affects navigation accuracy and makes the feature matching more difficult. As demonstrated, the Shi-Tomasi detector outperforms all others and maintains the best functionality.

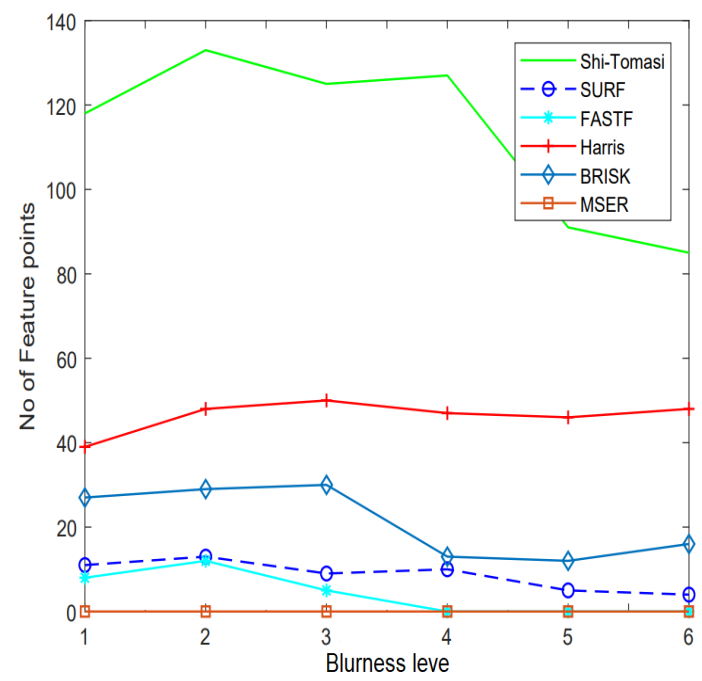

Figure. 3.9: Detectors' performance for different motion blur levels.

\section{White noise}

There are different sources of noise in space that could affect feature point detection. Hence, the performance of all feature detectors when images corrupted with different noise levels was evaluated. Figure. 3.10 shows the performance of the detectors in the presence of white noise with various means of $[0.1,0.2,0.3,0.4,0.5,0.6,0.7,0.8$, $0.9]$ and with different covariances $[0.01,0.02,0.03,0.04,0.05,0.06,0.07,0.08,0.09$, 0.1], as it is demonstrated increasing the mean does not have a significant impact on the detected number of feature points while large covariances are difficult for all the detectors. The performance of most of the detectors, particularly Fast, drops much farther while the Shi-Tomasi detector performed the best. 


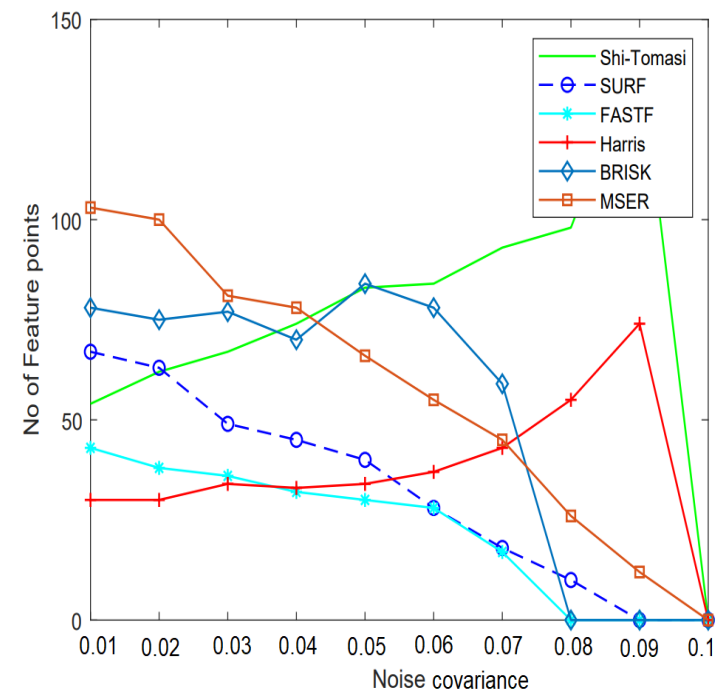

(a) Different noise covariances.

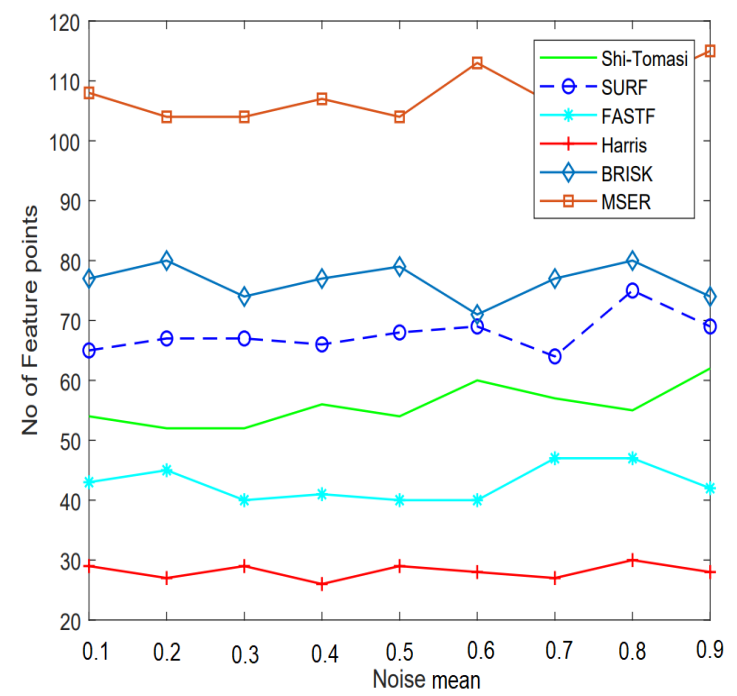

(b) Different noise mean

Figure. 3.10: Detectors' performance for different noise levels.

\section{Distance}

To evaluate the performance of detectors based on the distance to a target, images of a target captured at different distances while the chaser is moving toward the target spacecraft. For this experiment, the chaser spacecraft moved perpendicular toward the target, the detectors were evaluated for a relative distance of $[72,98,120,161] \mathrm{cm}$. Figure. 3.11 presents the obtained detector comparison results. As it shows, being close and far from the target reduces the detected feature points by all detectors. 


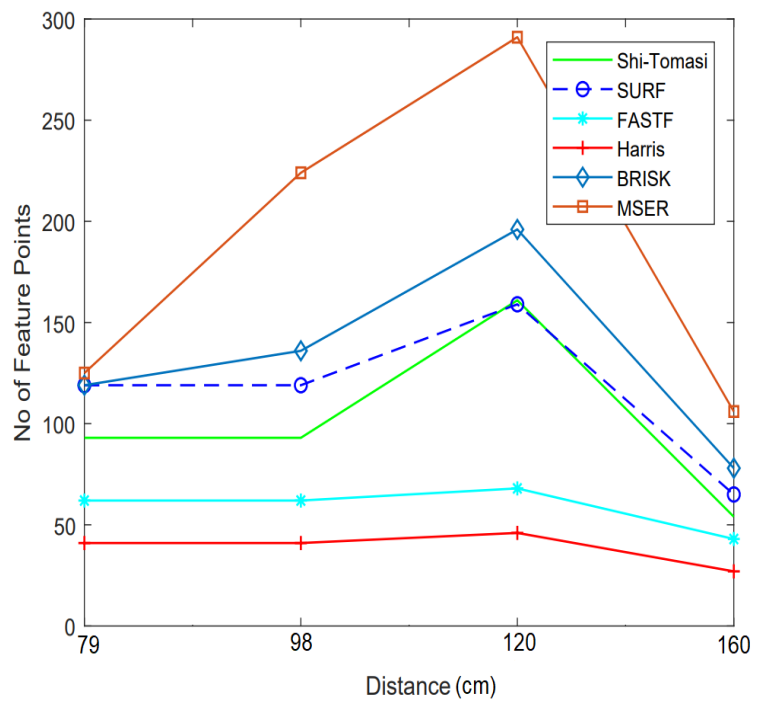

Figure. 3.11: Detectors' performance for different target's distances from the camera.

\subsubsection{Stereo Matching and 3-D Reconstruction}

Stereo matching is an essential aspect of computer vision. For the feature matching step, features are first extracted from the left and right images, then corresponding points in the rectified images that are projections of the same $3-\mathrm{D}$ point $M$ are identified. The RANSAC algorithm was used to remove stereo matching outliers. Figure. 3.12 shows the results of feature matching and outlier removal across rectified stereo images. With stereo cameras, objects in the field of view will appear to be slightly different locations in the two images due to the cameras' different scene perspectives. Depth information can be computed from a pair of stereo images by establishing the distance in pixels between the location of a feature in one image and its position in the other image. This provides a disparity map. 


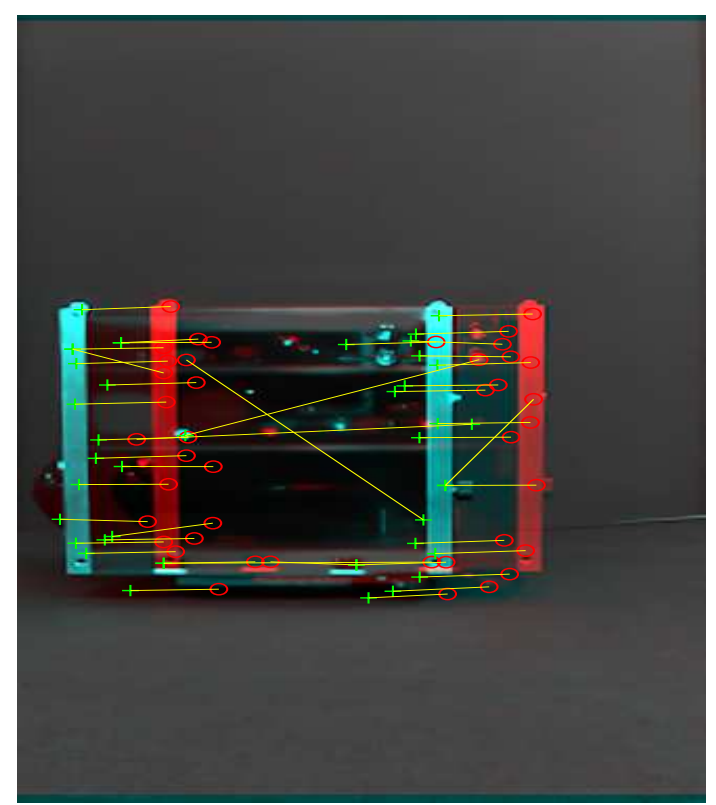

(a) Before outlier removal.

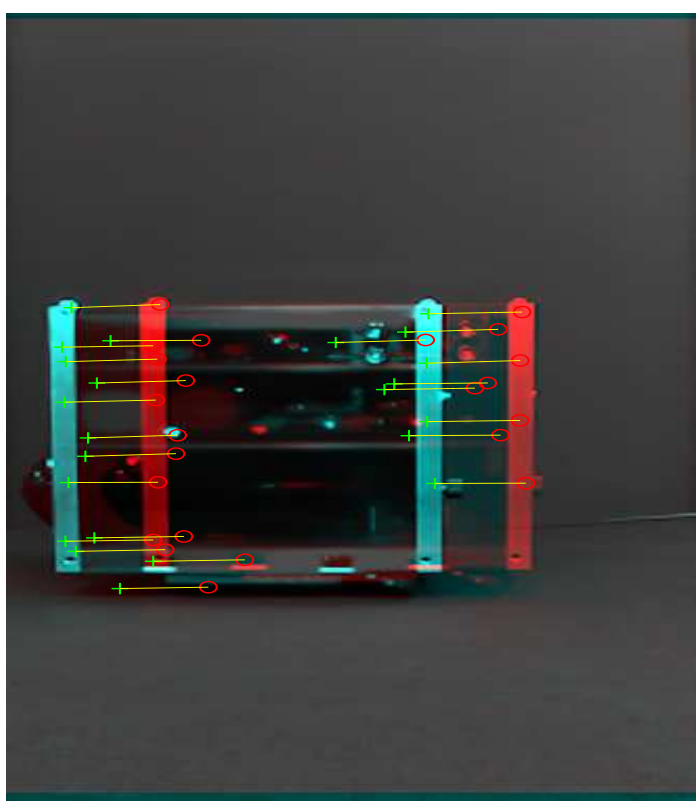

(b) After outlier removal.

Figure. 3.12: Matching and outlier removal.

\subsubsection{Disparity Map and Triangulation}

To compute the disparity map, the Sum of Absolute Differences (SAD) was selected to generates a disparity of the stereo image pairs from the two cameras, which can then be used for depth maps and 3-D positioning. The algorithm then determines the similarity between a square window in the left image and a corresponding window in the right image by calculating the absolute difference between each pixel in the left window and the corresponding pixels in the right window and compares them. The differences are summed to create a simple metric of block similarity. Specifically, given an image (in which each pixel $(u, v)$ corresponds to a projection of a threedimensional point onto the camera focal plane) with a grayscale intensity value of $I(u, v)$, the error function can be computed as: 


$$
\Phi_{S A D}(u, v)=\sum_{x=-M_{1}}^{M_{1}} \sum_{y=-M_{2}}^{M_{2}}\left|I_{L}(u+x+d, u+y)-I_{R}(u+x, u+y)\right|
$$

where $\left(2 M_{1}+1\right) \times\left(2 M_{2}+1\right)$ is the size of the window, $I_{L}$ and $I_{R}$ are the intensity of pixels in the left and right camera images, respectively. $d$ is the disparity (i.e., the positional difference of a given pixel), and its value can then be optimized by minimizing the SAD error function. The optimized disparity is denoted by:

$$
d^{*}(u, v)=\arg \min \Phi_{S A D}(u, v)
$$

This block-matching algorithm is known for its high performance, and the confidence estimate can be used to mask out regions of high noise effectively. The selection of block size is a trade-off between disparity map noise and accuracy.

\section{Triangulation}

Once the disparity map is computed and the reconstructed 3-D points from their projections in two images, the points are triangulated to compute a three-dimensional point cloud, as follows:

$$
\begin{gathered}
Z_{i}=T_{x} * f / u-v \\
X_{i}=C_{x}-u * Z_{i} / f \\
Y_{i}=v-C_{y} * Z_{i} / f
\end{gathered}
$$

where $\left[X_{i}, Y_{i}, Z_{i}\right]$ denote the position of a given point $i$ with respect to a reference frame, with its origin at the center of the left camera focal plane, and its z-axis parallel 
to the optical axis of the cameras. $f$ is the focal length of the left camera, and $C_{x}$ and $C_{y}$ are the principal point coordinates in pixels with $T_{x}$ as the baseline. The mean value of these point clouds is calculated and used to estimate the geometric center of the target object as follows:

$$
X_{C G}=1 / N \sum_{i=0}^{N} X_{i}, \quad Y_{C G}=1 / N \sum_{i=0}^{N} Y_{i}, \quad Z_{C G}=1 / N \sum_{i=0}^{N} Z_{i},
$$

The relative velocity is computed from the relative position using the first-order difference. The error results of three-dimensional relative position and velocity components for a spinning target spacecraft are provided in Figures. 3.13 and 3.14, respectively. These error signals were generated by comparing the outputs of the relative vision-based navigation system with ground truth data obtained with a tape measure. As shown in the figures, maximum position and velocity errors along each axis are in the order of $0.1 \mathrm{~m}$ and $0.04 \mathrm{~m} / \mathrm{s}$. Another experiment was performed to assess the performance at various distances along the line-of-sight axis, and the relative position was determined at four arbitrarily distances: [0.47, 0.64, 0.93, 1.29] m. As indicated in Figure. 3.15, the relative position error increases slightly as the distance increases because when the target is further away from the camera, there are fewer traceable features that the algorithm can use.

Assuming that the target spacecraft is a symmetrical and rotates only about its major axis of inertia, the position along the $Z$ component of the relative position vector will oscillate periodically back and forth, following a sinusoidal form. This is a reasonable assumption since, by virtue of the major-axis rule, a spin about the major axis of inertia is the only stable rotational motion. Indeed, a major-axis spin 
80
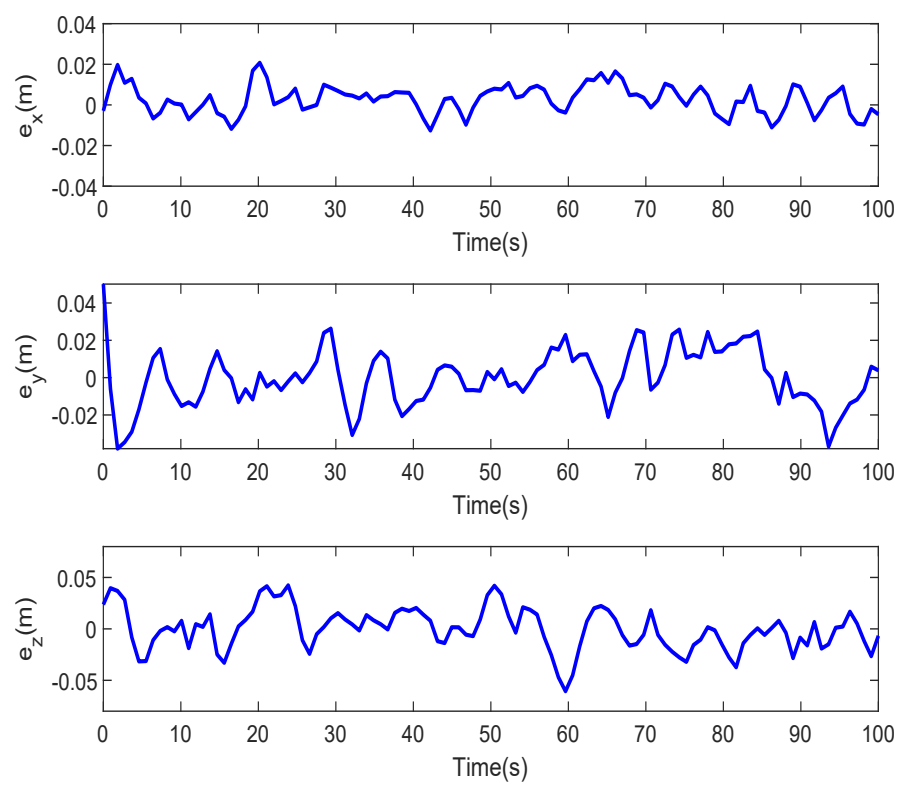

Figure. 3.13: Relative position errors.
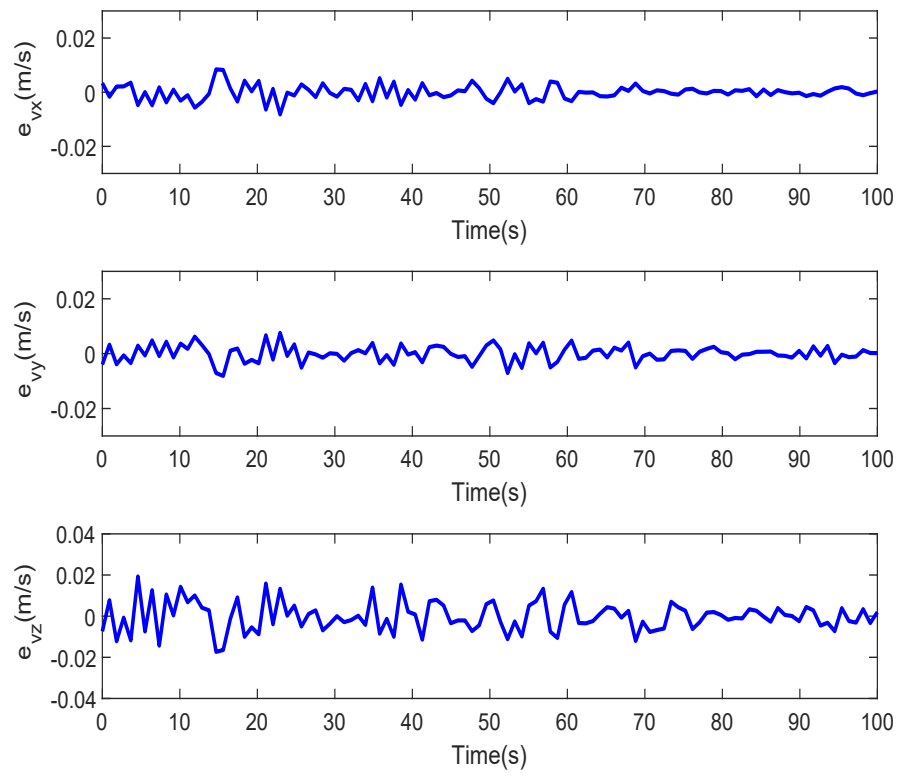

Figure. 3.14: Relative velocity errors. 


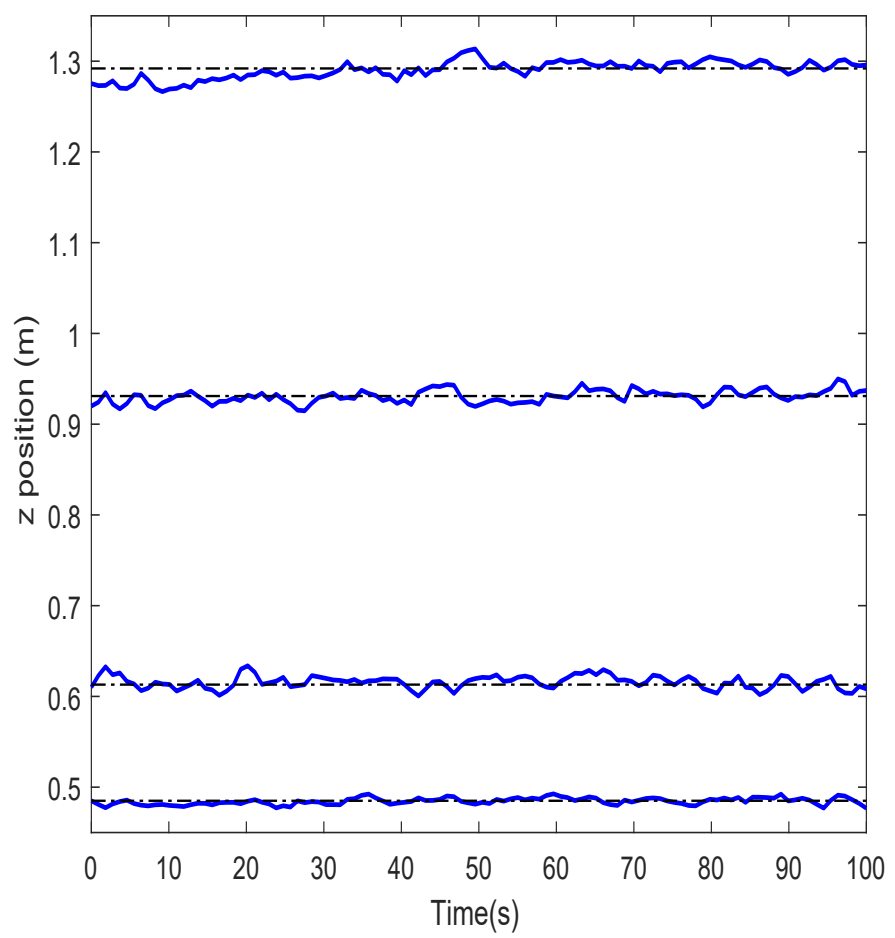

Figure. 3.15: Relative position at four different distances.

requires a minimum of kinetic energy, so most spacecraft will have a major-axis spin if given sufficient time in orbit due to energy dissipation. The angular velocity about the major axis of rotation can then be determined directly from the period of this cyclic variation in relative position measurements. Figure. 3.16 illustrates the angular velocity error about the major axis of rotation of a target spacecraft when spinning at 10.6 revolutions per minute $(\mathrm{rpm})$.

\subsection{Tracking}

Feature tracking is a key aspect of many vision-based applications. After object detection, it is crucial to track the object from one frame to the next, mainly for target pose and motion estimation purposes. However, some environmental conditions, such 


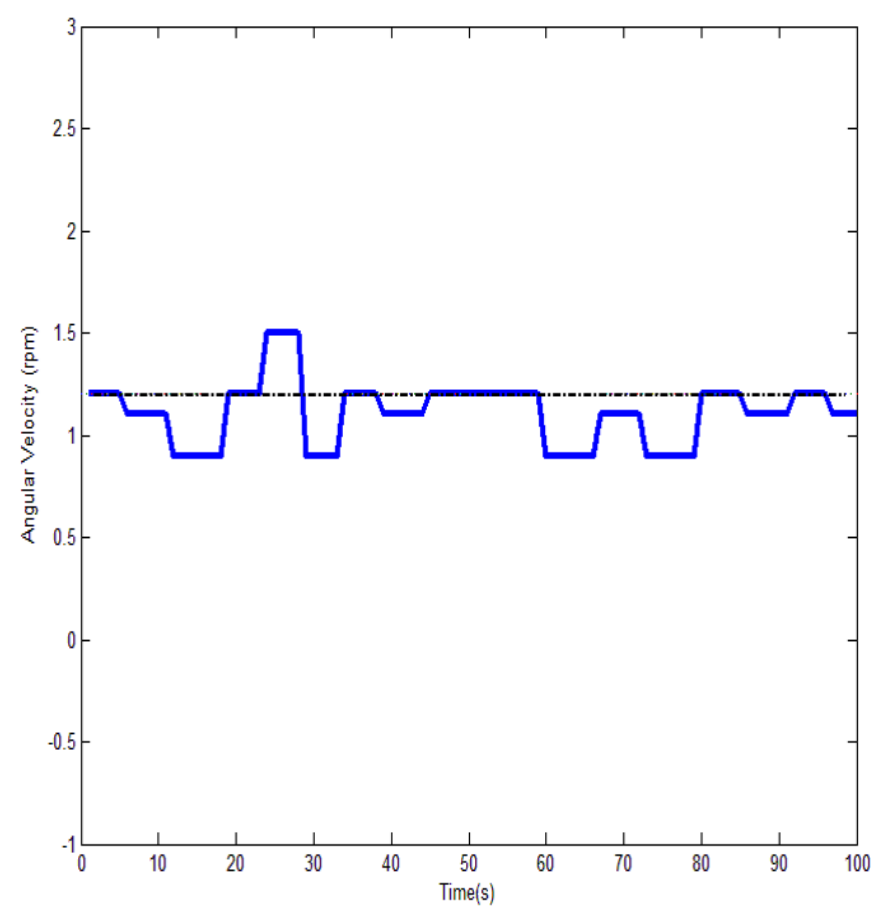

Figure. 3.16: Angular velocity error about the major axis of rotation.

as intense illumination and distortion, can make feature detection and tracking very challenging. Here, a new adaptive Kalman filter has been developed for tracking and estimating feature point positions in video frames. The diagram of the tracking process is shown in Figure. 3.17. Once the feature points in the first frame are detected and extracted, the initial conditions are reconstructed for the estimation process; with the uncertainty range and location of feature points in the $k^{\text {th }}$ frame, the UKF predicts the location and uncertainty of the feature points in the $(k+1)^{t h}$ frame. The observed locations of feature points in the $(k+1)^{t h}$ frame are acquired using the detected and extracted feature points. Finally, to achieve the optimal locations of the feature points and the uncertainty range, the feature point locations in the $(k+1)^{t h}$ frame are used to update the UKF. The fuzzy logic adaptation mechanism determines the value of 


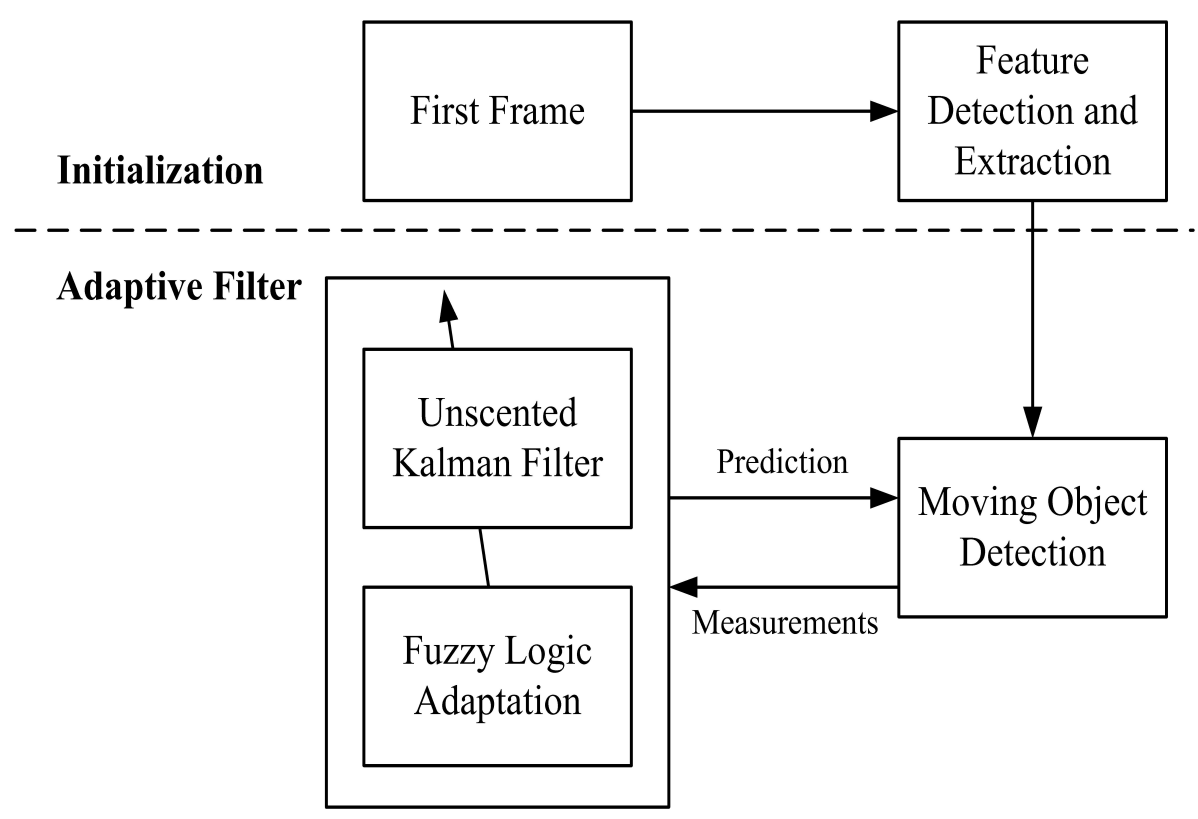

Fig. 3.17. The tracking process diagram.

the weighting factor for every frame to adjust UKF and prevent it from diverging.

\subsubsection{System Description}

The dynamic and measurement models are given by:

$$
\begin{gathered}
\boldsymbol{x}_{k}=\boldsymbol{f}\left(\boldsymbol{x}_{k-1}\right)+\boldsymbol{w}_{k} \\
\boldsymbol{y}_{k}=\boldsymbol{h}\left(\boldsymbol{x}_{k}\right)+\boldsymbol{v}_{k}
\end{gathered}
$$

where the vectors $\boldsymbol{x}_{k}$ and $\boldsymbol{y}_{k}$ are states of the system, and the observed measurement signal that driven by the noises $\boldsymbol{w}_{k}$ and $\boldsymbol{v}_{k}$, respectively. The constant velosity equations was used to reflect the dynamics of a feature points. The model for the $i^{t h}$ feature point is therefore defined as follows: 


$$
\begin{aligned}
& x_{i}(k)=x_{i}(k-1)+v_{i}(k)(\Delta t) \\
& y_{i}(k)=y_{i}(k-1)+v_{i}(k)(\Delta t)
\end{aligned}
$$

where $\Delta t$ is the time interval between the states.

The measurement model of the $i^{\text {th }}$ feature point observed by the camera creates an observation model and can be written as:

$$
\boldsymbol{y}_{i}=\boldsymbol{h}_{i}(\boldsymbol{x})+\boldsymbol{v}_{i}, \quad i=1, \ldots, N
$$

where $\boldsymbol{v}_{i}$ represent the measurement noise and $\boldsymbol{h}_{i}(\boldsymbol{x})$ defined as:

$$
\boldsymbol{h}_{i}(\boldsymbol{x})=\left[\boldsymbol{x}_{1}, \boldsymbol{y}_{1}, \boldsymbol{x}_{2}, \boldsymbol{y}_{2}, \ldots, \boldsymbol{x}_{N}, \boldsymbol{y}_{N}\right]^{T}
$$

where $\left\{\left(\boldsymbol{x}_{i}, \boldsymbol{y}_{i}\right)\right.$, for $\left.i=1,2, \ldots, N\right\}$ denote the perspective projection of the $i^{t h}$ detected feature point on a picture plane.

\subsubsection{EKF and UKF for Feature Tracking}

EKF is an optimal method that is widely applied for tracking. It is employed as a recursive structure that used actual motion parameters to correct the estimation of future points motion continues to improve the estimation accuracy [196]. EKF equations fall into two groups. Time prediction equation and measurement equations (update). The current state and the covariance error estimate from time update and the measurement update equations provide feedback. Table 3.3 provides the EKF implementation for a tracking purpose.

The extended Kalman filter (EKF) is probably the most widely used estimation 
Table 3.3: $\quad$ Tracking with EKF

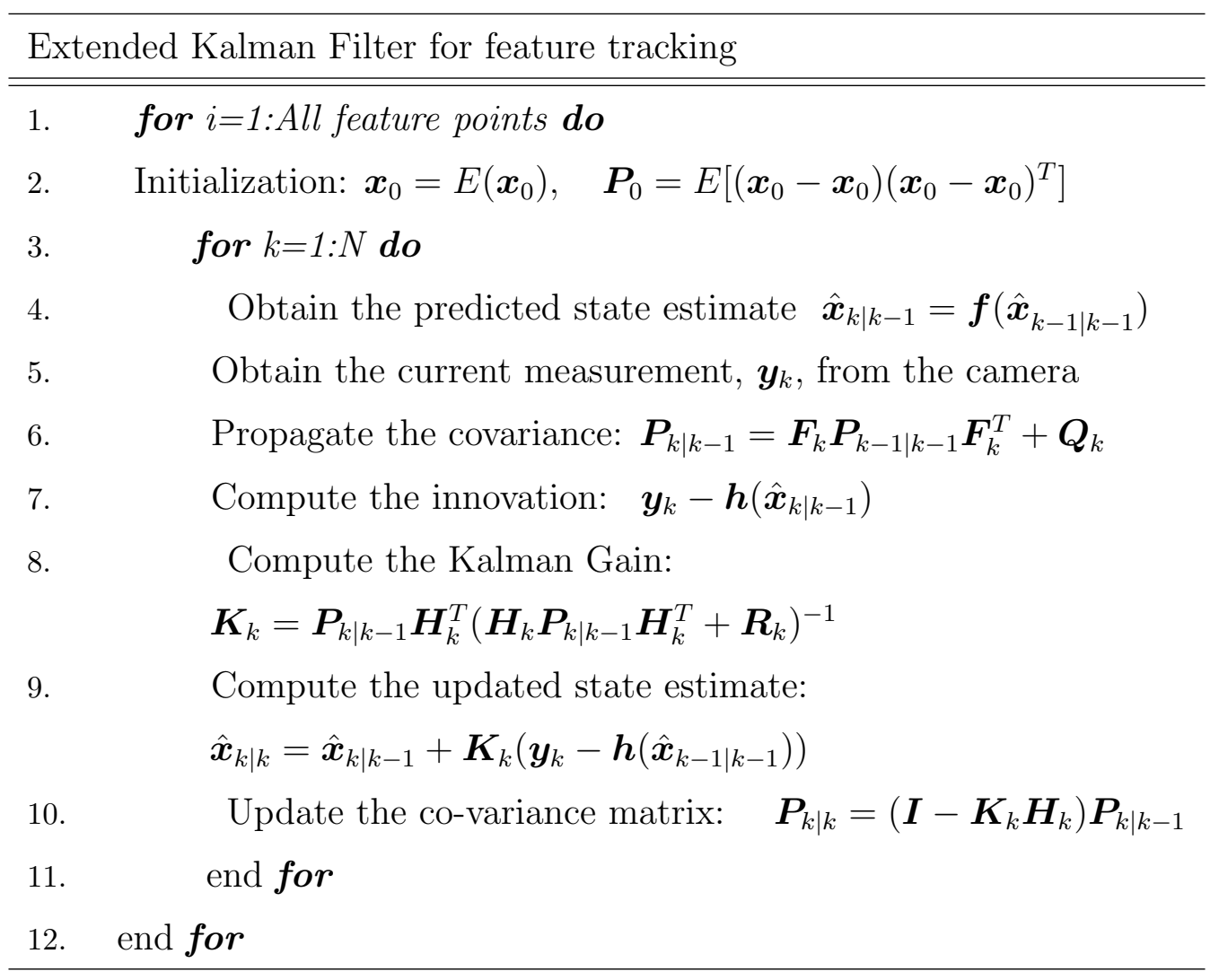

algorithm for nonlinear systems. However, more than 35 years of experience in the estimation community has shown that is difficult to tune, and only reliable for systems that are almost linear on the time scale of the updates. Many of these difficulties arise from its use of linearization. To overcome this limitation, the unscented transformation (UT) was developed as a method to propagate mean and covariance information through nonlinear transformations. It is more accurate, easier to implement, and uses the same order of calculations as linearization [197]. The UKF algorithm for feature-based tracking is summarized in Table 3.4. 
Table 3.4: Tracking with UKF

UKF for feature tracking

1. for $i=1$ :All feature points do

2. Initialization: $\hat{\boldsymbol{x}}_{0}=E\left(\boldsymbol{x}_{0}\right), \quad \boldsymbol{P}_{0}=E\left[\left(\boldsymbol{x}_{0}-\hat{\boldsymbol{x}}_{0}\right)\left(\boldsymbol{x}_{0}-\hat{\boldsymbol{x}}_{0}\right)^{T} \quad \lambda=\sigma^{2}(L+\kappa)-L\right.$

3. for $k=1: N$ do

4. $\quad$ Compute sigma points: $\boldsymbol{\chi}_{k-1}=\left[\begin{array}{ll}\hat{\boldsymbol{x}}_{k-1} & \hat{\boldsymbol{x}}_{k-1} \pm \sqrt{(L+\lambda)} \sqrt{\boldsymbol{P}_{k-1}}\end{array}\right]$

5. Obtain predicted state estimate : $\boldsymbol{\chi}_{k \mid k-1}^{(i)}=\boldsymbol{f}\left(\boldsymbol{\chi}_{k-1}\right), \quad i=0, \ldots, 2 L$

6. $\quad$ Propagate the mean: $\hat{\boldsymbol{x}}_{k \mid k-1}=\sum_{i=0}^{2 L} \eta_{i}^{(m)} \boldsymbol{\chi}_{k \mid k-1}^{(i)}$

7. Propagate the covariance:

$$
\boldsymbol{P}_{k \mid k-1}=\boldsymbol{Q}+\sum_{i=0}^{2 L} \eta_{i}^{(c)}\left(\boldsymbol{\chi}_{k \mid k-1}^{(i)}-\hat{\boldsymbol{x}}_{k \mid k-1}\right)\left(\boldsymbol{\chi}_{k \mid k-1}^{(i)}-\hat{\boldsymbol{x}}_{k \mid k-1}\right)^{T}
$$

8. Obtain the current step measurement $\boldsymbol{z}_{k}$ from the camera

$$
\hat{\boldsymbol{z}}_{k \mid k-1}=\sum_{i=0}^{2 L} \eta_{i}^{(m)} \boldsymbol{\gamma}_{k \mid k-1}^{(i)}, \quad \boldsymbol{\gamma}_{k \mid k-1}^{(i)}=\boldsymbol{h}\left(\boldsymbol{\chi}_{k \mid k-1}^{(i)}\right)
$$

9. $\quad$ Compute the covariance matrix:

$$
\boldsymbol{P}_{k}^{z z}=\sum_{i=0}^{2 L} \eta_{i}^{(c)}\left(\boldsymbol{\gamma}_{k \mid k-1}^{(i)}-\hat{\boldsymbol{z}}_{k \mid k-1}\right)\left(\boldsymbol{\gamma}_{k \mid k-1}^{(i)}-\hat{\boldsymbol{z}}_{k \mid k-1}\right)^{T}
$$

10. Compute the cross-covariance matrix:

$$
\boldsymbol{P}_{k}^{x z}=\sum_{i=0}^{2 L} \eta_{i}^{(c)}\left(\boldsymbol{\chi}_{k \mid k-1}^{(i)}-\hat{\boldsymbol{x}}_{k \mid k-1}\right)\left(\boldsymbol{\gamma}_{k \mid k-1}^{(i)}-\hat{\boldsymbol{z}}_{k \mid k-1}\right)^{T}
$$

11. Compute the Kalman Gain: $\boldsymbol{K}_{k}=\boldsymbol{P}_{k}^{x z}\left(\boldsymbol{P}_{k}^{z z}\right)^{-1}$

12. Compute the updated state estimate: $\hat{\boldsymbol{x}}_{k}=\hat{\boldsymbol{x}}_{k \mid k-1}+\boldsymbol{K}_{k}\left(\boldsymbol{z}_{k}-\hat{\boldsymbol{z}}_{k \mid k-1}\right)$

13. Update the covariance matrix $\boldsymbol{P}_{k}=\boldsymbol{P}_{k \mid k-1}-\boldsymbol{K}_{k} \boldsymbol{P}_{k}^{z z} \boldsymbol{K}_{k}^{T}$

14. end for

15. end for

\subsubsection{Fuzzy Adaptive UKF}

A fundamental problem in autonomous navigation and motion estimation is automatically detecting and tracking features in consecutive frames, challenging when camera motion is significant. Besides, the reliability of the motion estimation can be degraded by a variety of factors, including changes in illumination, poor image contrast, occlusion of features, or unmodeled changes in objects. In the case of an uncooperative space target such as space debris, the actual trajectory is unknown. 
In addition, due to harsh space conditions and the target's motion, feature tracking is very challenging. As a result optimized feature tracking mechanism is essential. Here, to manages these shortcomings, a Fuzzy adaptive mechanism based on UKF is developed. To adjust the UKF covariance matrices and prevent the filter from divergence, tuning parameters $\alpha$ and scale are used to increase the accuracy. The proposed Weighted Unscented Kalman filter can be described as equations that follow below.

The first step in implementing the UKF is to create the sigma-points throughout the scaled Unscented Transformation (UT). For $L$ element state vector, the UT generates $(2 L+1)$ sigma vector $\chi$ as:

$$
\begin{gathered}
\boldsymbol{\chi}_{0}=\hat{\boldsymbol{x}}_{k-1} \\
\boldsymbol{\chi}_{k-1}=\hat{\boldsymbol{x}}_{k-1}+\sqrt{(L+\lambda) \boldsymbol{P}_{k-1}} \\
\boldsymbol{\chi}_{k-1}=\hat{\boldsymbol{x}}_{k-1}-\sqrt{(L+\lambda) \boldsymbol{P}_{k-1}}
\end{gathered}
$$

where $\boldsymbol{P}_{k-1}$ is the error covariance of the state vector, $\hat{\boldsymbol{x}}_{k-1}$ denotes the estimated state and $L$ is the length of the state vector. The scaling parameter $\lambda$ is given by:

$$
\lambda=\sigma^{2}(L+\kappa)-L
$$

The scaling parameter $\sigma, 0 \leq \sigma \leq 1$, defines the size of the sigma-points distribution. The scaling parameter $\kappa \geq 1$ influences the accuracy of the approximation. It should be noted that the scaling parameters are typically assumed constant through the estimation procedure. Once the sigma-points have been generated, each point is propagated throughout the state equation as: 


$$
\chi_{k \mid k-1}^{(i)}=\boldsymbol{f}\left(\boldsymbol{\chi}_{k-1}^{(i)}\right), \quad i=0,1, \ldots, 2 L
$$

The mean is approximated by the mean of the transformed sigma-points as follows:

$$
\hat{\boldsymbol{x}}_{k \mid k-1}=\sum_{i=0}^{2 L} \eta_{i}^{(m)} \boldsymbol{\chi}_{k \mid k-1}^{(i)}
$$

The covariance matrix is modified by multiplying the weighting parameter $\alpha$ as follows:

$$
\boldsymbol{P}_{k \mid k-1}=\boldsymbol{Q} \alpha^{-2}+\sum_{i=0}^{2 L} \eta_{i}^{(c)}\left(\boldsymbol{\chi}_{k \mid k-1}^{(i)}-\hat{\boldsymbol{x}}_{k \mid k-1}\right)\left(\boldsymbol{\chi}_{k \mid k-1}^{(i)}-\hat{\boldsymbol{x}}_{k \mid k-1}\right)^{T}
$$

Due to the additive noise assumption, the process noise covariance matrix $\alpha^{-2}$ is multiplied by the covariance matrix. The $\eta_{i}^{(m)}$ and $\eta_{i}^{(c)}$ terms denote the mean weight vector and covariance weight vector; respectively, and are defined as:

$$
\begin{gathered}
\eta_{0}^{(m)}=\lambda /(L+\lambda) \\
\eta_{0}^{(c)}=\lambda /(L+\lambda)+\beta-\sigma^{2}+1 \\
\eta_{i}^{(c)}=\eta_{i}^{(m)}=1 /(2(L+\lambda)) \quad i=1,2, \ldots, 2 L
\end{gathered}
$$

The $\beta$ parameter denotes a non-negative weighting parameter employed to include prior distribution information. The transformed set of sigma points throughout the measurement equation are given as:

$$
\gamma_{k \mid k-1}^{(i)}=\boldsymbol{h}\left(\chi_{k \mid k-1}^{(i)}\right)
$$


Then, the mean of the measurement vector is calculated as:

$$
\hat{\boldsymbol{z}}_{k \mid k-1}=\sum_{i=0}^{2 L} \eta_{i}^{(m)} \boldsymbol{\gamma}_{k \mid k-1}^{(i)}
$$

The cross-covariance and covariance matrices of the weighted UKF are defined as:

$$
\begin{gathered}
\boldsymbol{P}_{k}^{x z}=\alpha^{-2} \sum_{i=0}^{2 L} \eta_{i}^{(c)}\left(\boldsymbol{\chi}_{k \mid k-1}^{(i)}-\hat{\boldsymbol{x}}_{k \mid k-1}\right)\left(\boldsymbol{\gamma}_{k \mid k-1}^{(i)}-\hat{\boldsymbol{z}}_{k \mid k-1}\right)^{T} \\
\boldsymbol{P}_{k}^{z z}=\boldsymbol{R} \alpha^{-2}+\sum_{i=0}^{2 L} \eta_{i}^{(c)}\left(\boldsymbol{\gamma}_{k \mid k-1}^{(i)}-\hat{\boldsymbol{z}}_{k \mid k-1}\right)\left(\boldsymbol{\gamma}_{k \mid k-1}^{(i)}-\hat{\boldsymbol{z}}_{k \mid k-1}\right)^{T}
\end{gathered}
$$

where $\alpha$, denotes the turning parameter determined by the fuzzy logic based adaptation mechanism. The Kalman gain is defined as:

$$
\boldsymbol{K}_{k}=\boldsymbol{P}_{k}^{x z}\left(\boldsymbol{P}_{k}^{z z}\right)^{-1}
$$

The estimated state and covairance are updated by using the Kalman gain as follows:

$$
\begin{gathered}
\hat{\boldsymbol{x}}_{k}=\hat{\boldsymbol{x}}_{k \mid k-1}+\boldsymbol{K}_{k}\left(\boldsymbol{z}_{k}-\hat{\boldsymbol{z}}_{k \mid k-1}\right) \\
\boldsymbol{P}_{k}=\boldsymbol{P}_{k \mid k-1}-\boldsymbol{K}_{k} \boldsymbol{P}_{k}^{z z} \boldsymbol{K}_{k}^{T}
\end{gathered}
$$

where, $\hat{\boldsymbol{x}}_{k}$ and $\boldsymbol{P}_{k}$ are the posterior state and covariance estimates. The term $\boldsymbol{z}_{k}-$ $\hat{\boldsymbol{z}}_{k \mid k-1}$ is defined as the innovation sequence (residual). The innovation information can be used to determine the degree of divergence. The UKF becomes unstable when the innovation sequence is not zero mean and becomes larger; in such case, by applying an appropriate weighting factor, the fuzzy logic adaptation mechanism seeks to keep the innovation sequence acting as zero-mean white Gaussian noise. 


\section{Fuzzy logic adaptation scheme}

The implementation of the UKF requires prior information on both the process and measurement models. Inadequate measurement and model information can significantly degrade UKF performance and make the filter unstable [198]. In general, since there is no effective communication and no cooperation markers on the target, prior knowledge of the space targets cannot be achieved. To fulfill the requirement, an adaptive mechanism can be applied to adjust the parameters. This sub-section presents the Fuzzy logic system design. The key idea is to take advantage of the characteristics of fuzzy systems, such as simplicity of approach and the capability to deal with imprecise information. If the UKF is based on a complete and precise tuned model, the residual should be a zero-mean white noise process. If the residual is not white noise and correlated with itself, the UKF will diverge or coverage to a large bound [199]. The purpose of the adaptation mechanism here is to detect the divergences, adjust the weighting factors to tune UKF online to avoid divergence. The role of the matrices $\boldsymbol{Q}_{k}$ and $\boldsymbol{R}_{k}$ in any Kalman filters are to adjust the Kalman gain. The covariance matrix $\boldsymbol{Q}_{k}$ represents the uncertainty in the process model; an increase in the covariance matrix $\boldsymbol{Q}_{k}$ implies less confidence in the process model and more confidence in the measurement. The measurement noise covariance matrix $\boldsymbol{R}_{k}$ represents the precision of the measurement sensors, with a larger $\boldsymbol{R}_{k}$ of measured data indicating less confidence in this data and more confidence in the prediction. The residual of the filter can be employed to detect divergence. Therefore, the covariance and mean values of the residuals are used as inputs of the adaptation mechanism to determine the degrees of divergence of the filter and the exponential weighting $\alpha$ 


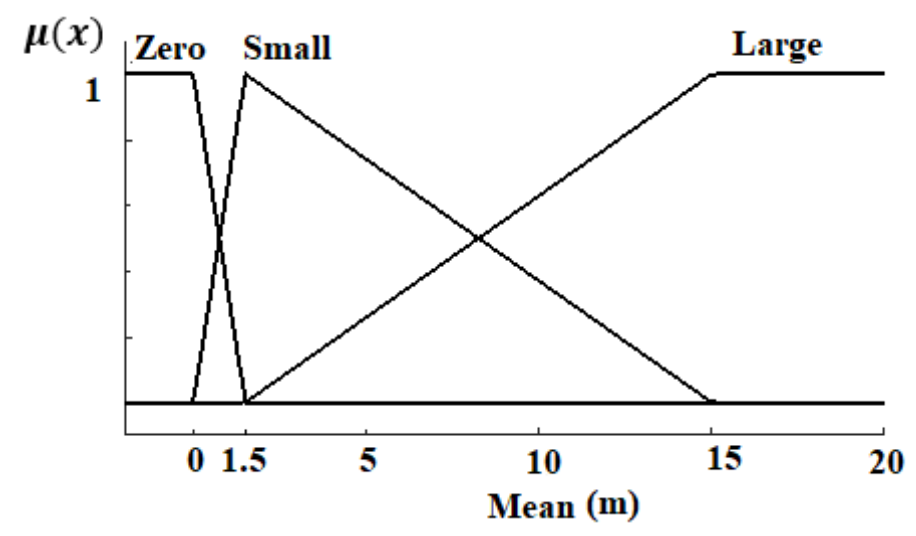

Figure. 3.18. Mean value membership functions.

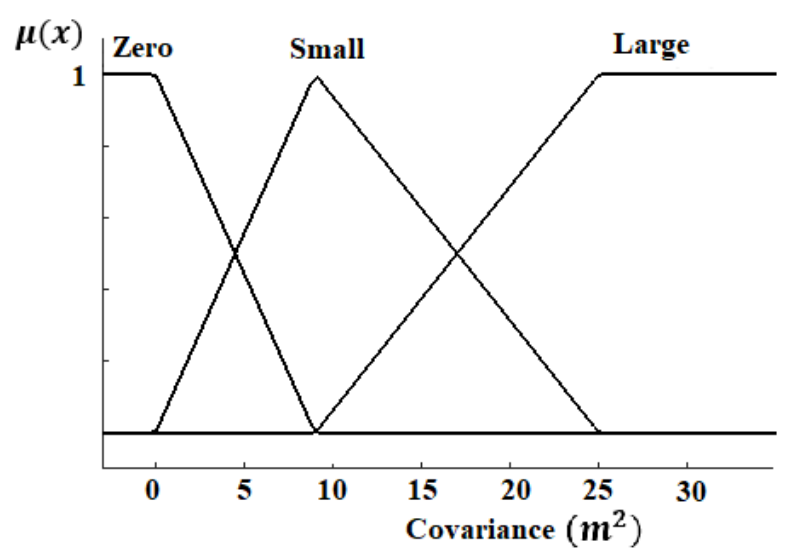

Figure. 3.19. Covariance membership functions.

defined as the output of the adaptation scheme.

The membership function of two fuzzy system inputs (mean and covariance of residual) and output of the first system, $\alpha$, are illustrated in Figures. 3.18, 3.19, and 3.20, respectively. The characteristics of a fuzzy system are highly dependent on the fuzzy rules. This particular adaptation scheme uses nine rules as outlined in Table 3.5. Once the covariance of the residual becomes high and the mean value becomes greater than zero, a larger $\alpha$ is applied to overcome the divergence due to the high uncertainty. If the residual covariance becomes very high, this indicates a problem with the measurement, which implies that the filter could not rely upon the 


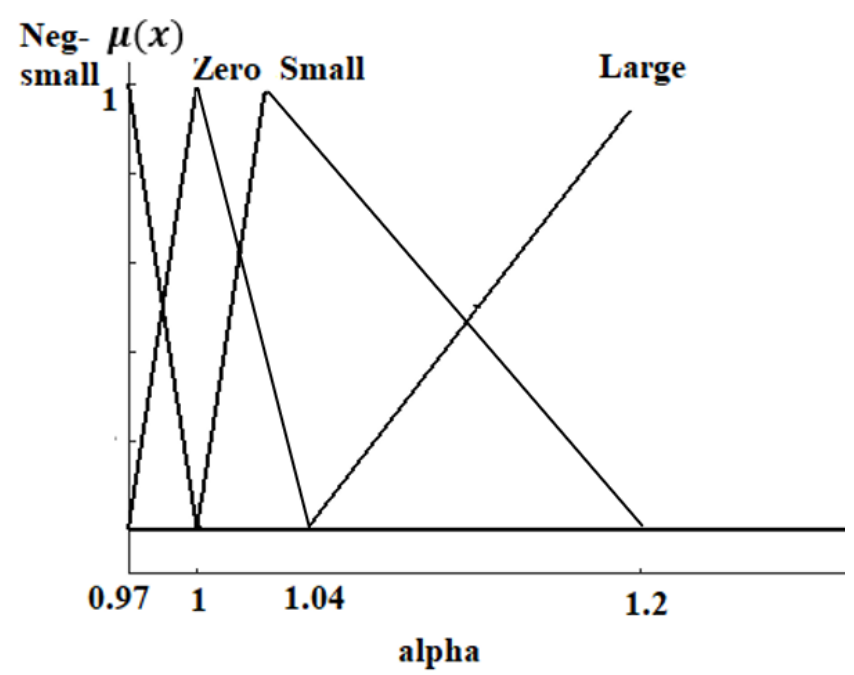

Figure. 3.20. $\alpha$ membership functions.

Table 3.5: Rule table of $\alpha$.

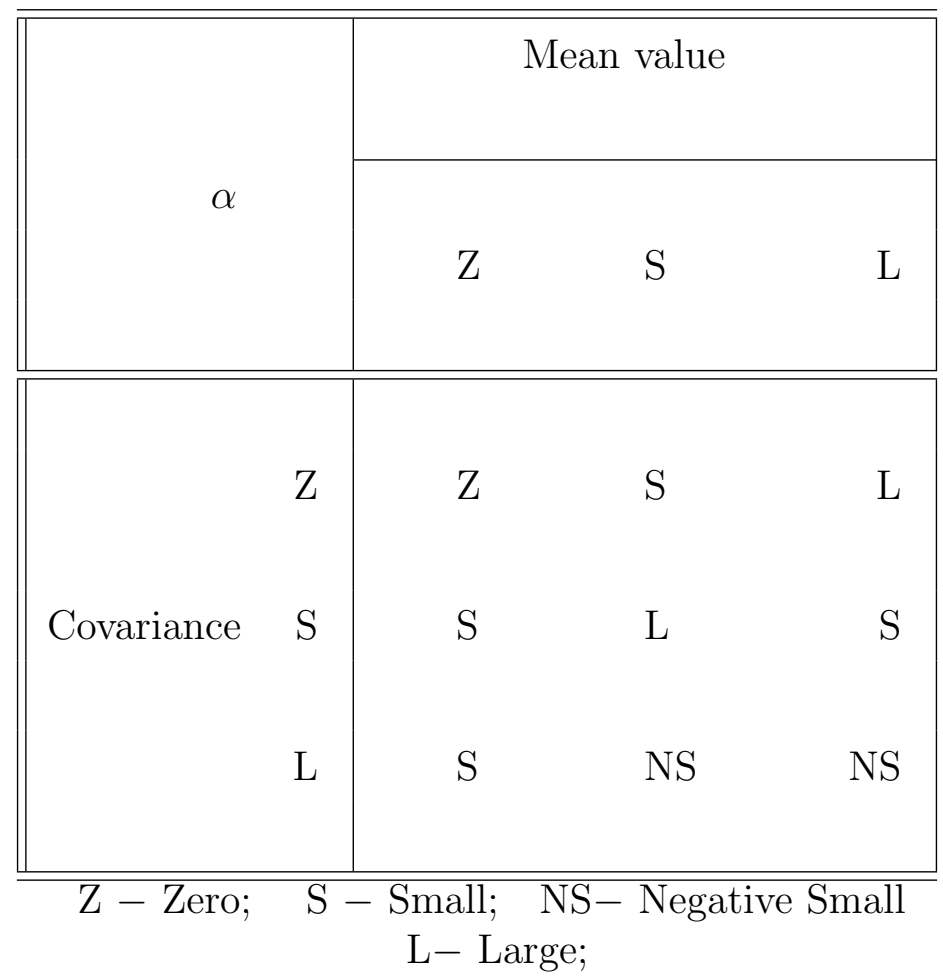

measurement. As a result, a smaller $\alpha$ is applied. The UKF works best when an appropriate tuning parameter is selected via the fuzzy adaptation mechanism, and it maintains the residual as zero-mean white noise. By selecting an appropriate tuning 


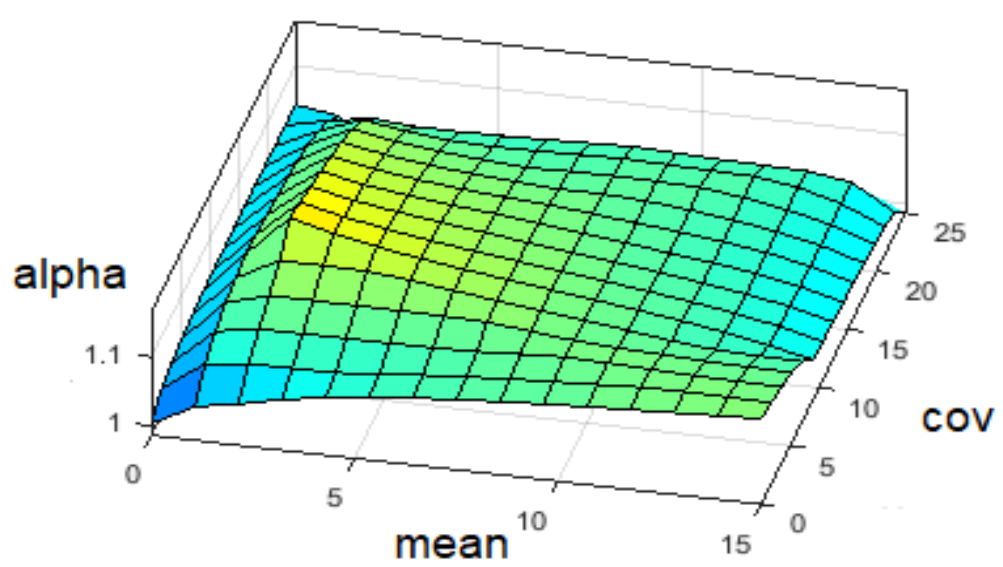

Figure. 3.21. Surface plot of the fuzzy logic system.

parameter via the fuzzy adaptation mechanism and keep the residual acting as a zero-mean white noise, the UKF can work optimally. The covariance matrix $\boldsymbol{R}_{k}$ is related to the covariance of the residual; therefore, any changes in the covariance matrix $\boldsymbol{R}_{k}$ will change the covariance of residual. To tune the covariance matrix $\boldsymbol{R}_{k}$, this system adapts the Kalman filter by selecting an appropriate scale. For instance, when the adaptation mechanism determines that the covariance of the residual is more significant than expected, it applies a proper scale to adjust $\alpha$. Similar to the previous fuzzy system, the covariance and mean values of the residuals are used as input, and scale is defined as the output. The scale was designed to adjust the $\alpha$. This fuzzy logic-based adaptation mechanism uses nine rules as shown in Table 3.6. The scale and $\alpha$ are related through the following equation.

$$
\alpha_{\text {new }}=(\alpha-1) \times \text { scale }+1
$$


Table 3.6: Rule table of scale.

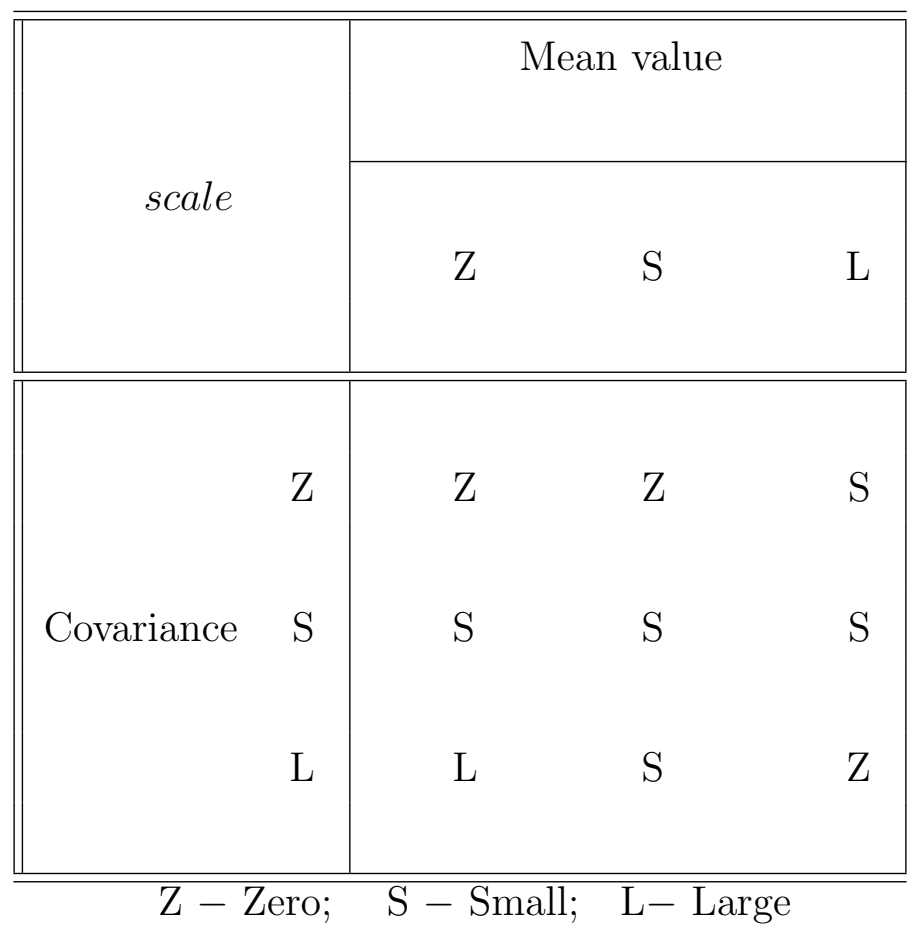

\subsection{Experimental Results}

\subsubsection{Using Testbed at the Spacecraft Robotics and Control Laboratory of Carleton University}

Different experiments have been conducted to evaluate the performance of the proposed fuzzy adaptive scheme. In the first experiment, the testbed at the spacecraft robotics and control laboratory of Carleton university was used to assess the tracking algorithm. One platform operates as a target spacecraft in this experiment, spinning around its major axis. The other platform (chaser) captures the image of the target with the stereo camera mounted on it. To track the captured feature, the chaser spacecraft moves in a circular path around the target spacecraft with a relative distance of $60 \mathrm{~cm}$. Figure. 3.22 shows the experimental setup. The suggested FAUKF, 


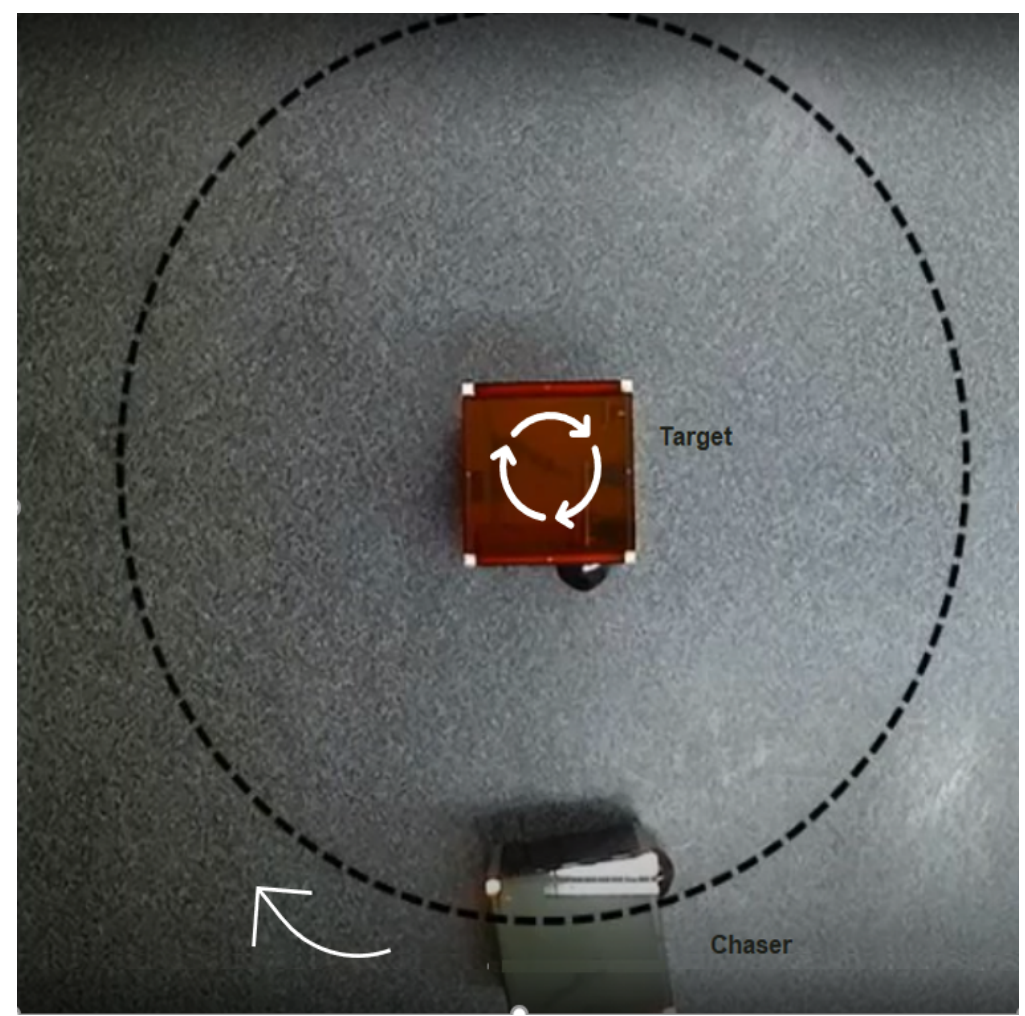

Figure. 3.22. Experimental setup at Carleton University spacecraft and control laboratory.

EKF and UKF were applied to track points of interest over video frames.

When feature points are selected in the first frame they are used for the entire tracking process, which can accelerate model updating significantly and reduce computational complexity. Target pose estimation with $P$ degrees of freedom requires a minimum of $P / 2$ feature points, so at least four feature points must be tracked over the frames to acquire the homograph correctly [200]. Figure. 3.23 shows the estimated positions of four feature points by EKF, UKF, FAUKF in comparison with the actual position (i.e., ground truth measured in real-time by an eight-camera motion capture system) of feature points. Figure. 3.29 shows the tracking errors of four feature points on the target rotating around its major axis. As illustrated, of these three approaches, the FAUKF demonstrates superior tracking accuracy performance compared to the 
others. The FAUKF tracking error is less than that of standard UKF and EKF.

Figure. 3.25 represents the actual trajectory of the feature point during spinning motion of the target spacecraft around its major axis and the estimated trajectory by filters FAUKF, UKF, and EKF. The proposed adaptive mechanism estimates a more accurate trajectory compared to other state-of-the-art filters.

One of the main issues with feature-based tracking is losing feature points, which can occur when a feature that has been tracked in previous images is no longer detected. This happens most frequently when features move outside of the camera's field of view. Many factors, including camera motion, illumination, abrupt object motion, and non-rigid object structure can cause missing feature points. To address missed tracking, the suggested algorithm searches the area predicted by FAUKF, and if no matched feature point is found, the processing step is skipped. In this case, an observed location from the camera was used as the predicted location, and the process repeated until the feature points appeared. If a feature point still cannot be found after $\mathrm{M}$ repeats, it is considered lost, and the tracking process moves on to replace other feature points. Target initialization is another significant problem that must be considered. All tracking algorithms require an initialization mechanism when the target appears for the first time; it can be automatic or manual, depending on the application [136]. This work used the automatic method for target initialization. Once feature points are detected in the first frame, they are considered the initial conditions for the estimation process. Here the algorithm was also tested under occlusion. It is assumed that a feature point that has been tracked is not detected in some video frames. EKF, UKF, and proposed FAUKF used to estimate feature points 

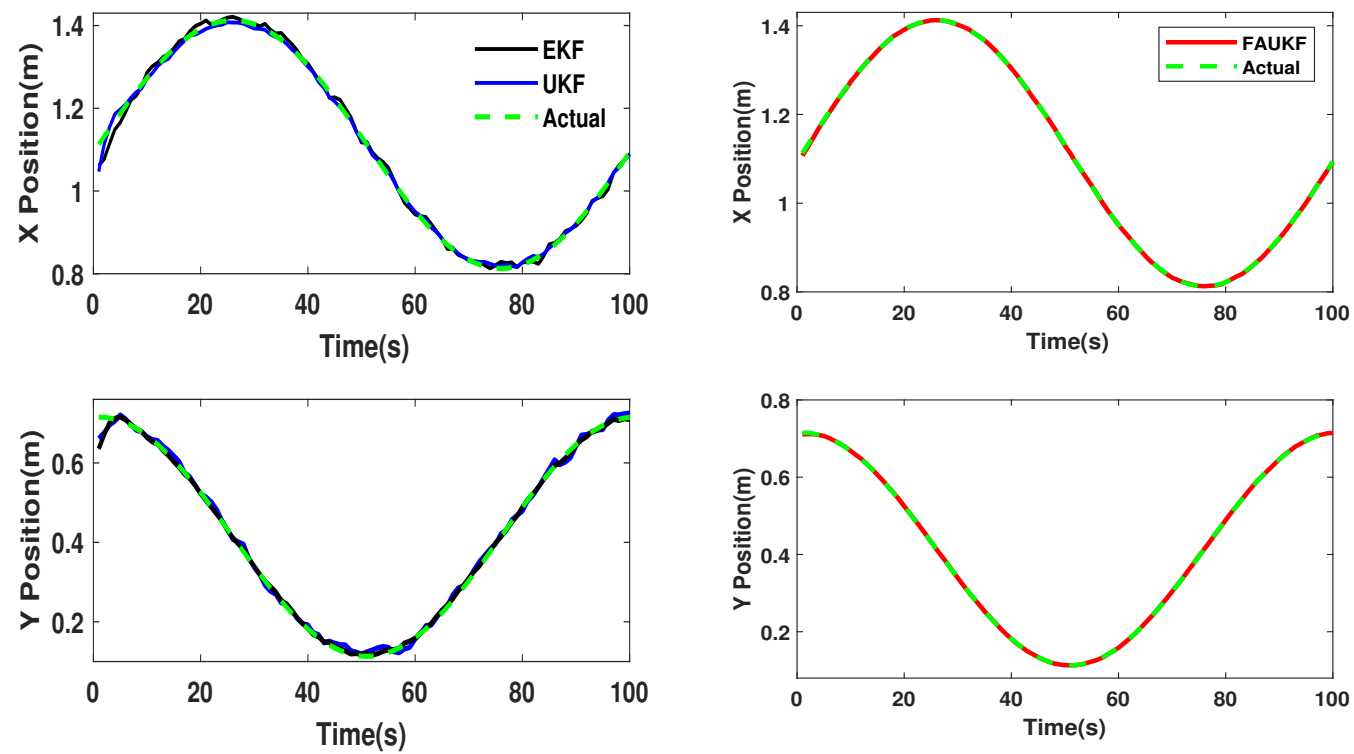

(a) EKF, UKF and actual locations of of Fea- (b) FAUKF and actual locations of of Feature ture point 1. point 1.
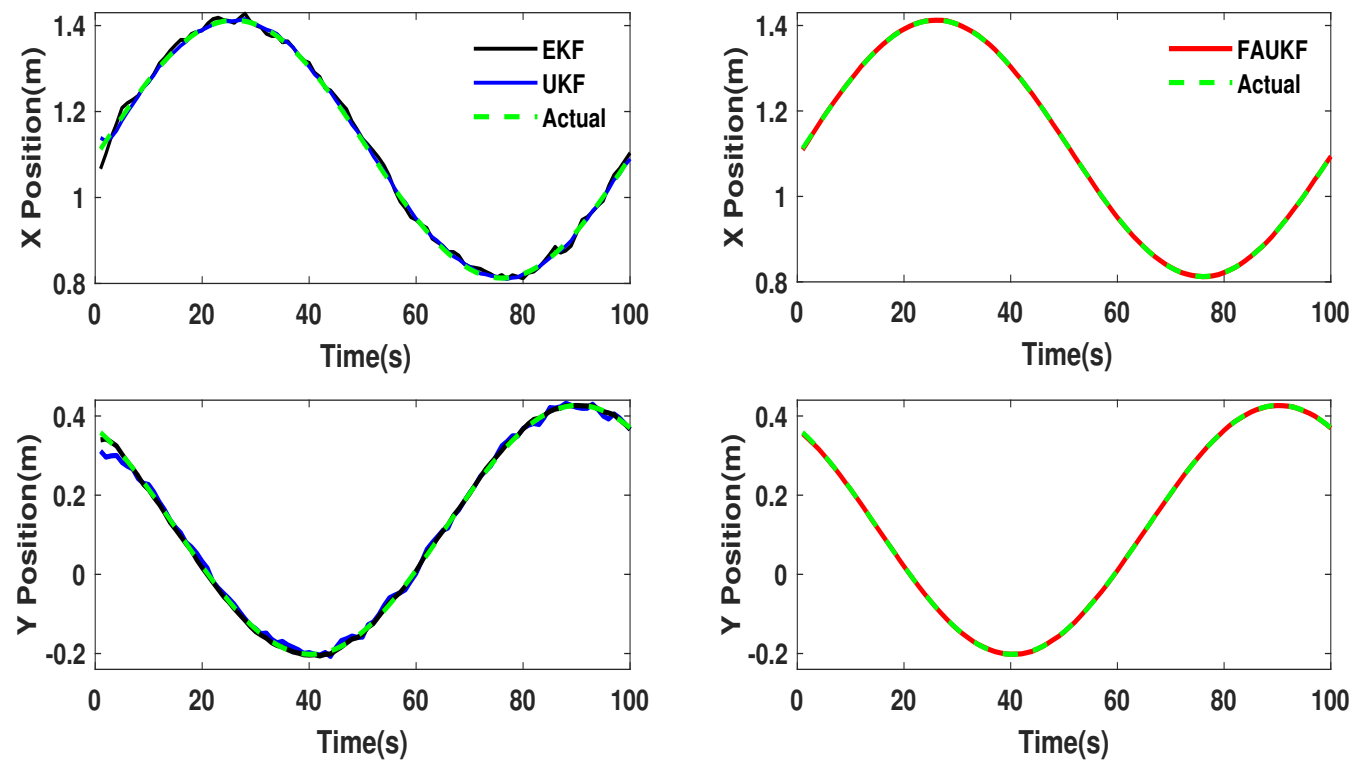

(c) EKF, UKF and actual locations of of Fea- (d) FAUKF and actual locations of of Feature ture point 2 . point 2 . 

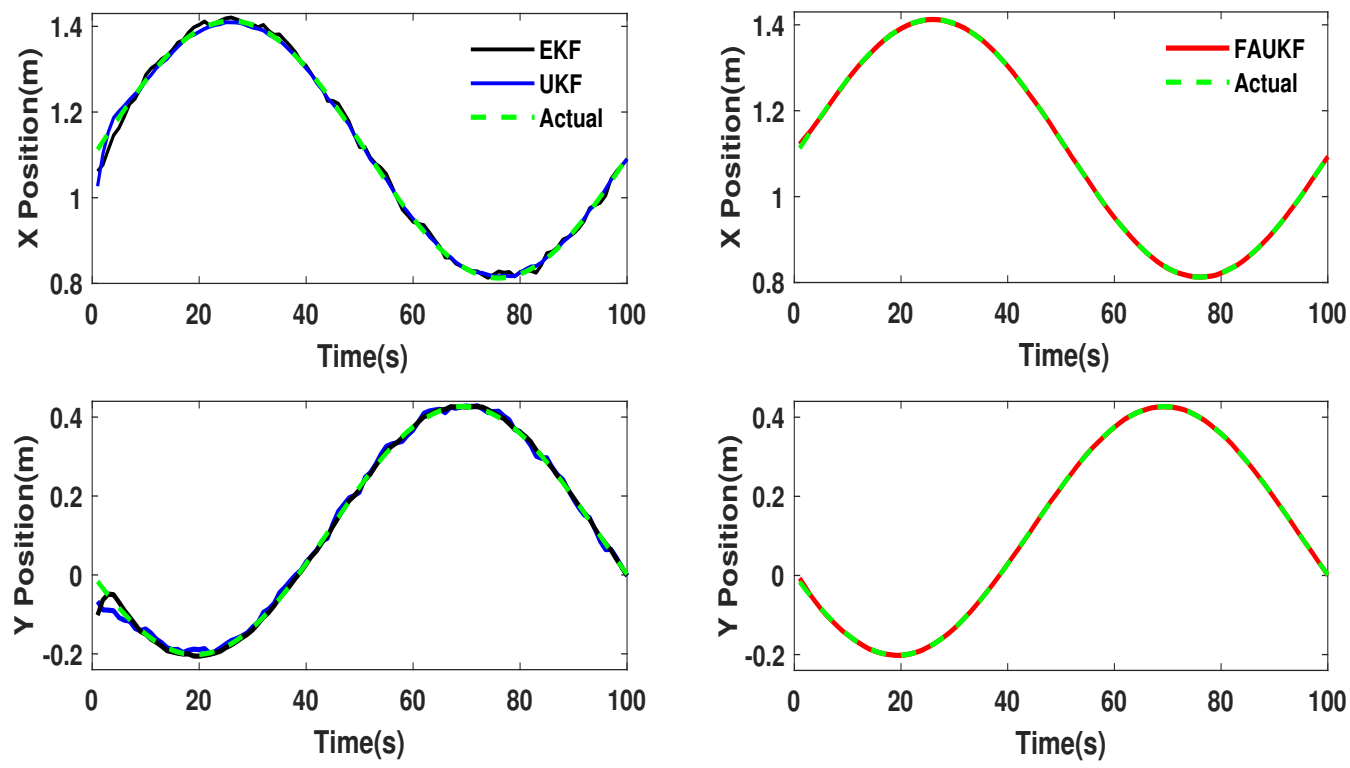

(e) EKF, UKF and actual locations of of Fea- (f) FAUKF and actual locations of of Feature ture point 3 . point 3 .
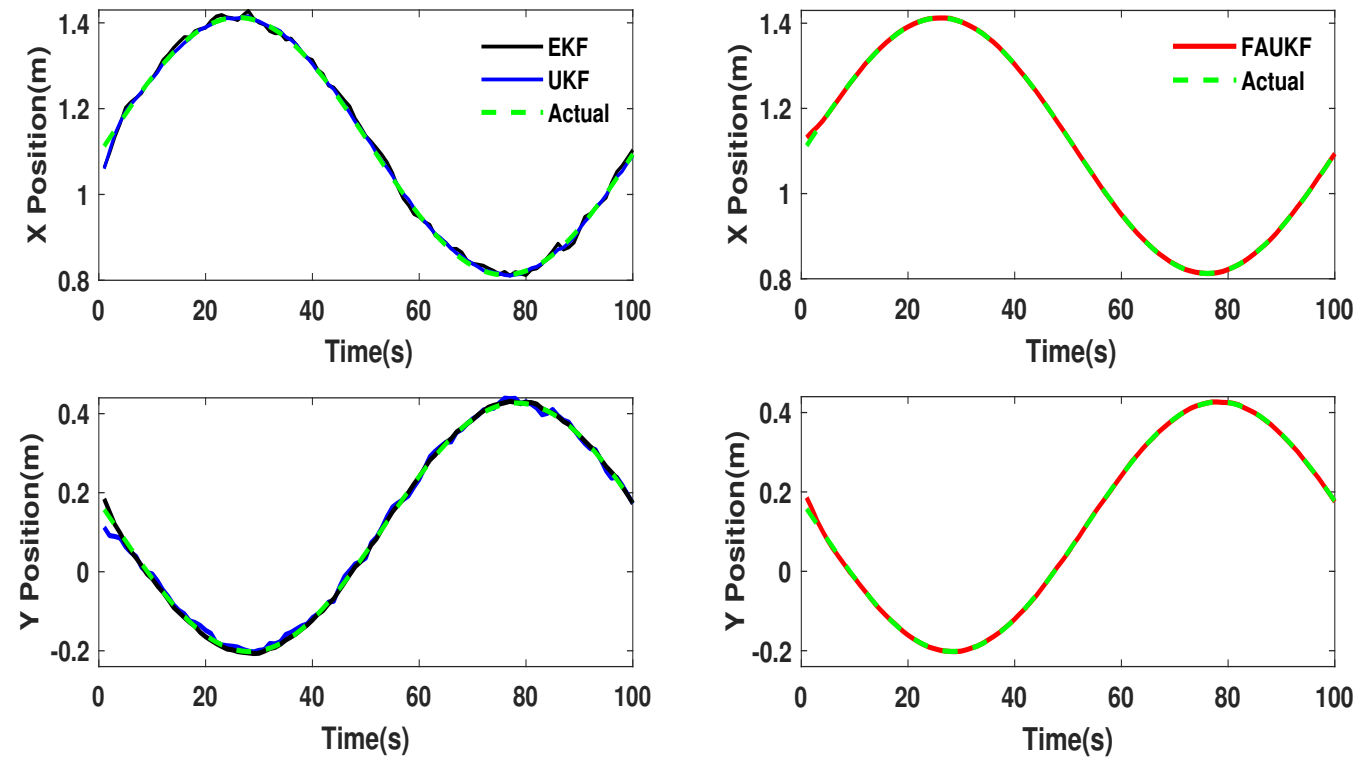

(g) EKF, UKF and actual locations of of Fea- (h) FAUKF and actual locations of of Feature ture point 4 . point 4 .

Figure. 3.23. Positions of feature points on the spinning target. 

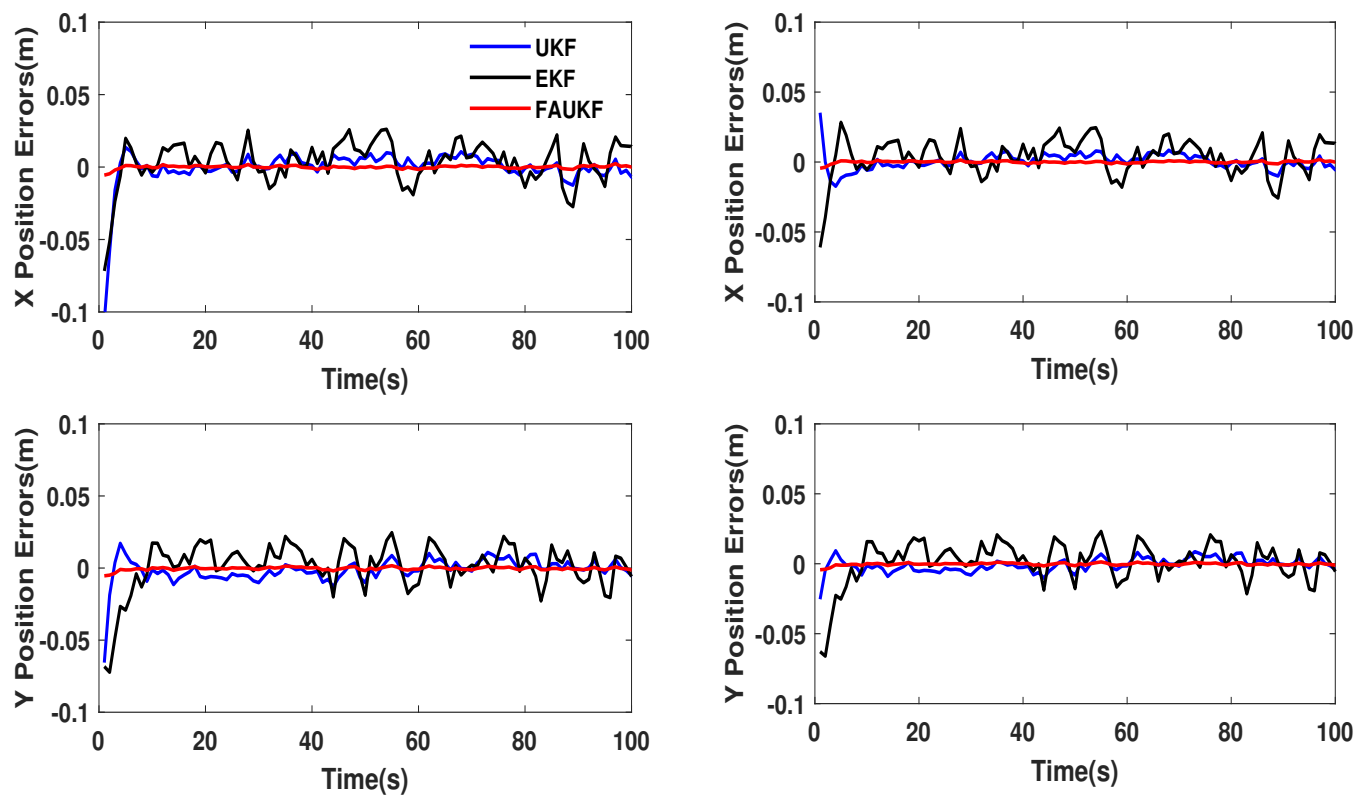

(a) Position errors of feature point 1.

(b) Position errors of feature point 2.
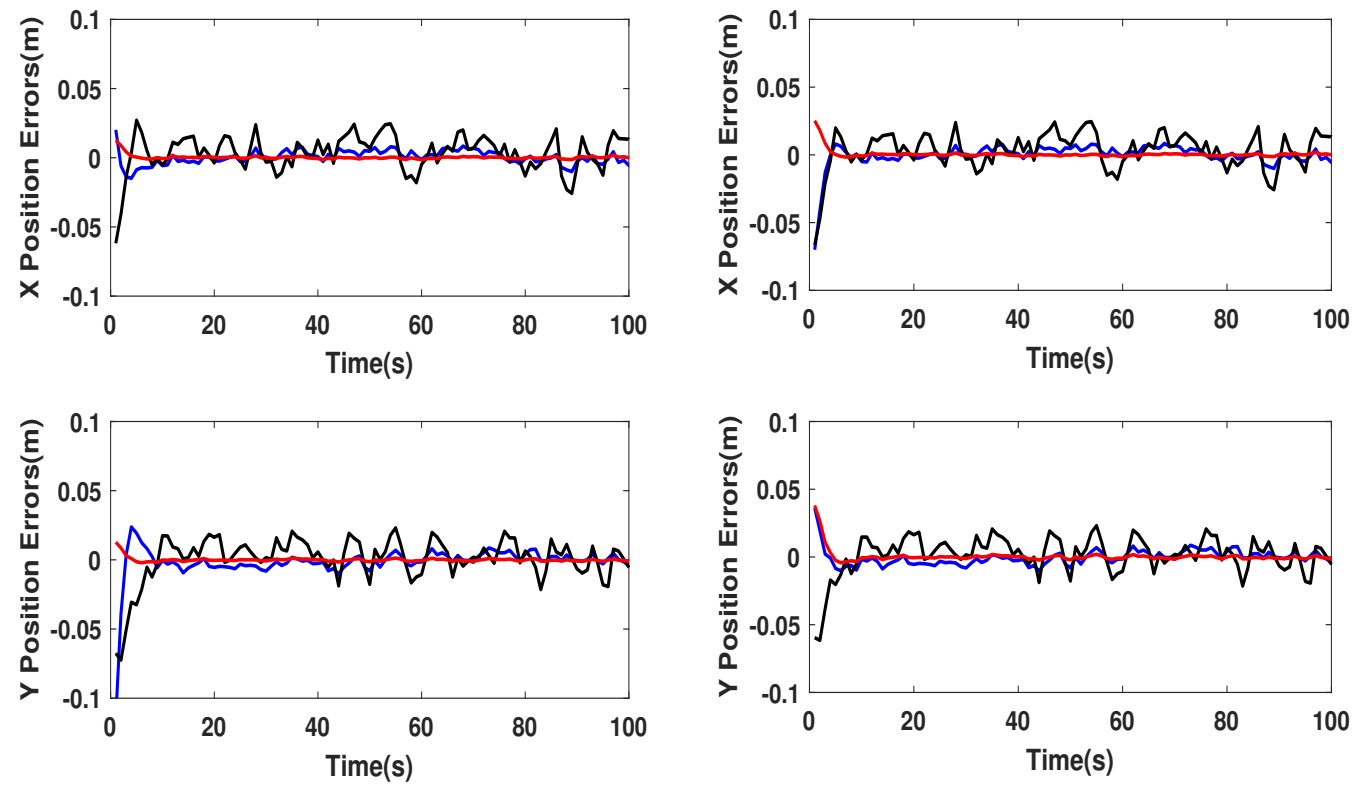

(c) Position errors of feature point 3.

(d) Position errors of feature point 4.

Figure. 3.24. Feature points' position errors for a spinning target. 


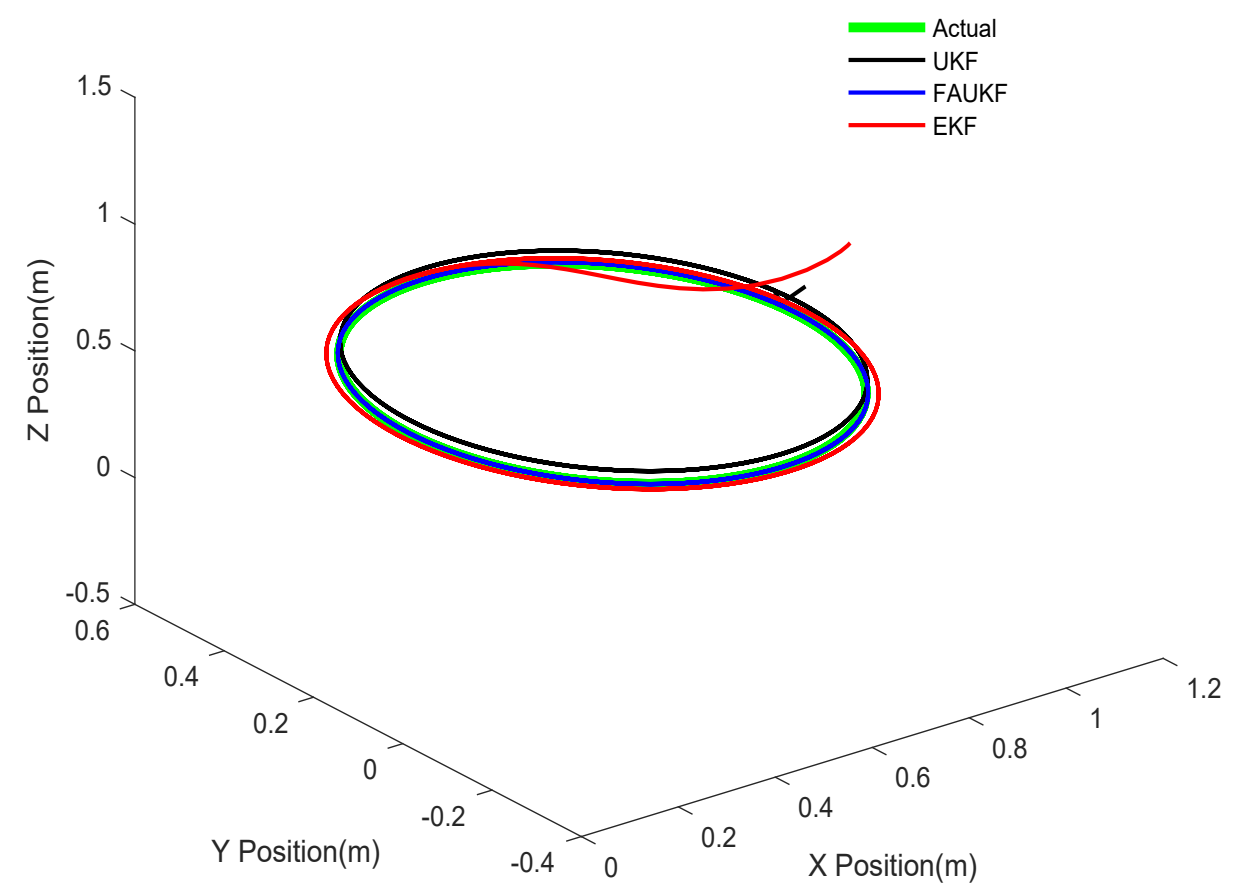

Figure. 3.25. The trajectory path of the $i^{t h}$ feature point during the spinning motion.

locations under complete occlusion. Figure. 3.26 presents the camera measurements of a feature point. As illustrated, in the case of occlusion, a feature point disappears during maneuvering. As a result, the camera is not able to measure the feature point positions. The actual (ground truth) and estimated position of the feature point using UKF are presented in Figure. 3.27, and Figure. 3.28 shows the estimating performance of FAUKF, as well as the actual position of the feature point. As shown FAUKF has superior tracking accuracy performance compared to UKF when the feature point is missing in some frames. Due to the poor performance of EKF, the results are not presented here. In another experiment, the three filters were tested with occlusion duration of $3,5,10,15$, and 30 frames. Table 3.7 provides a summary of RMS errors 
Table 3.7: The position RMS errors for different duration of occlusion.

\begin{tabular}{ccccccc}
\hline \hline & \multicolumn{7}{c}{ RMS ERROR (m) } \\
\cline { 2 - 7 } & EKF & \multicolumn{7}{c}{ X } & Y & X & Y & X & Y \\
\hline No of Frames=3 & 0.059 & 0.045 & 0.049 & 0.044 & 0.006 & 0.004 \\
No of Frames=5 & 0.061 & 0.063 & 0.051 & 0.058 & 0.007 & 0.005 \\
No of Frames=10 & 0.183 & 0.147 & 0.082 & 0.082 & 0.018 & 0.016 \\
No of Frames=20 & 0.383 & 0.249 & 0.144 & 0.125 & 0.087 & 0.083 \\
No of Frames=30 & 0.519 & 0.642 & 0.474 & 0.3512 & 0.099 & 0.089 \\
\hline \hline
\end{tabular}

for the three configurations. As indicates, FAUKF had the lowest tracking error compared to the other filters. The results demonstrated that long-lasting occlusions are very difficult to handle. However, if the maneuvering object moves in the same direction at uniform velocity, longer occlusion can be managed. The computation time of the three filters is summarized in Table 3.8.

Table 3.8: Computational complexity.

\begin{tabular}{lccl}
\hline \hline Filter & EKF & UKF & FAUKF \\
\hline Computational Time [spf] & 0.00031 & 0.00260 & 0.00540 \\
\hline \hline
\end{tabular}

\subsubsection{Using Actual Images from STS-131 Mission}

A different dataset has been used to evaluate the performance of the proposed fuzzy adaptive scheme. Figure. 3.29 shows the tracking results of the proposed FAUKF for nine different video frame samples. The tracking error of all three filters for four feature points is shown in Figure. 3.30. Of the three approaches, FAUKF demonstrates 

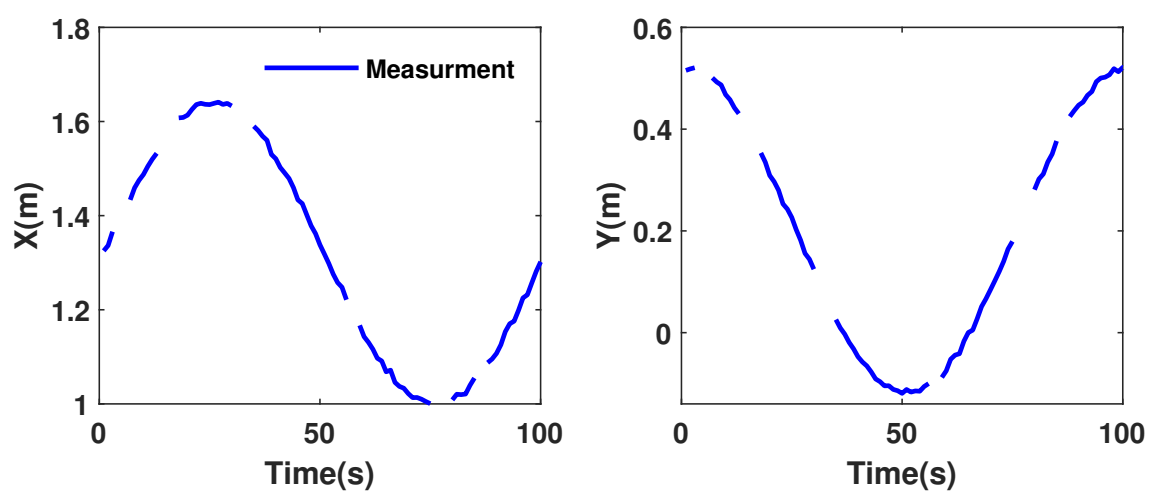

Figure. 3.26. Measurement data.
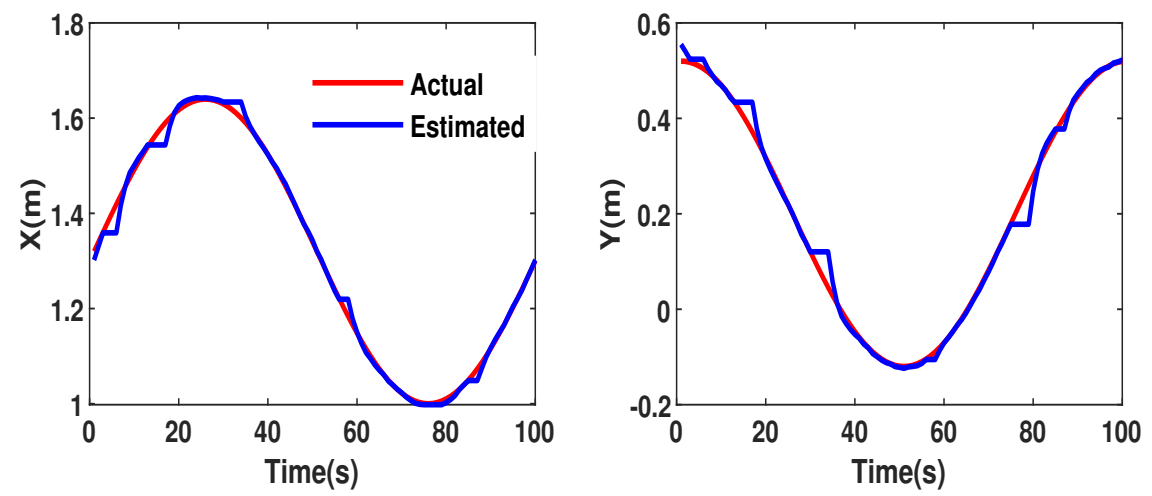

Figure. 3.27. UKF motion estimation.
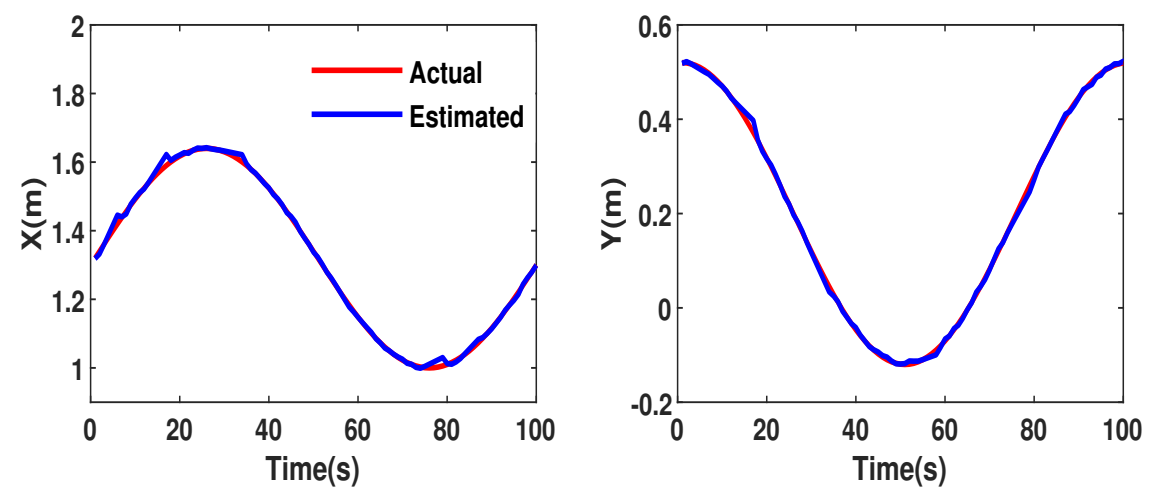

Figure. 3.28. FAUKF motion estimation. 
superior tracking accuracy performance compared to the other filters. The FAUKF tracking error is lower than that of standard UKF and EKF. It should be noted that this dataset includes the tracking ground truth for each frame.
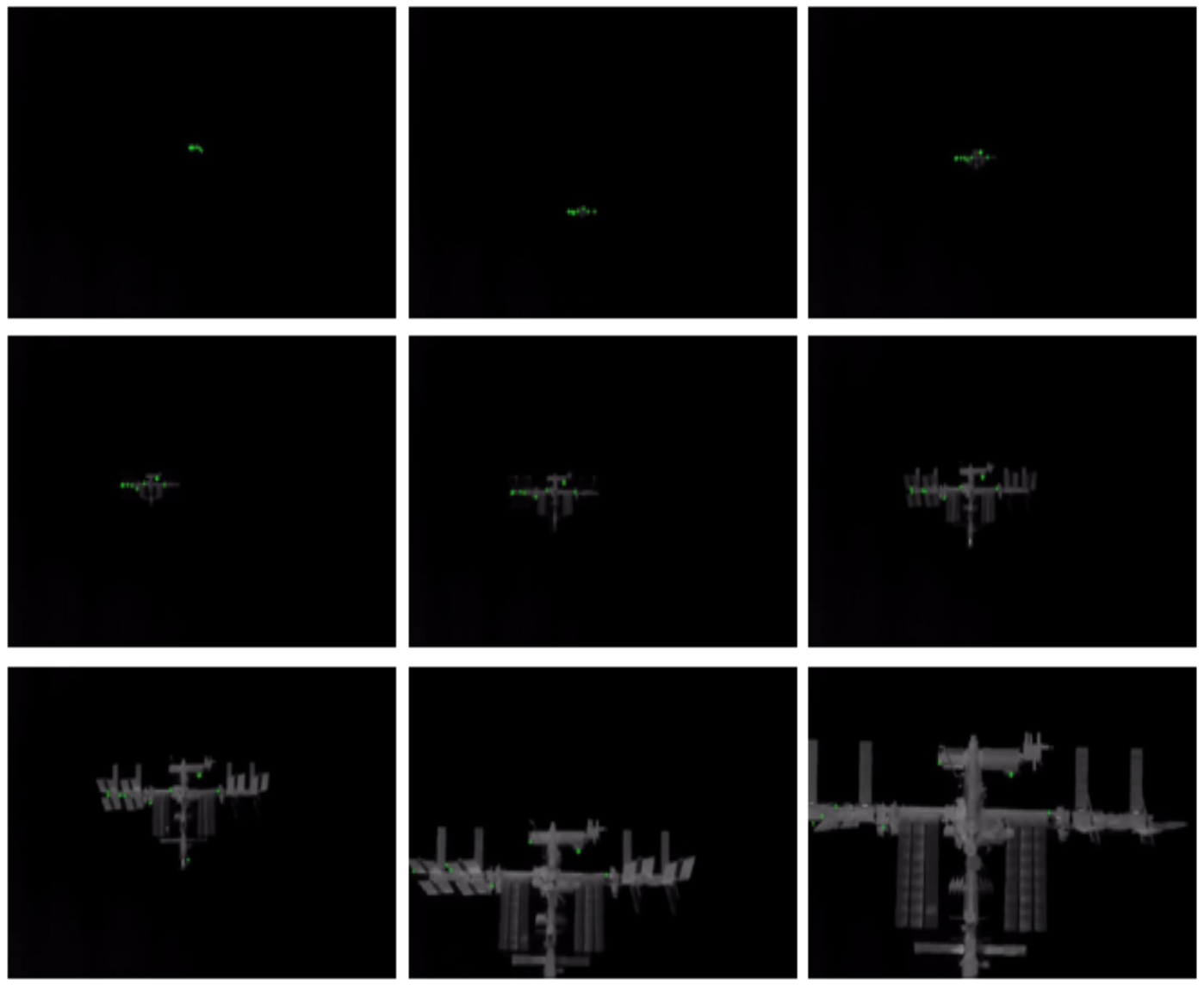

Figure. 3.29. Tacking samples, the first row from left to right the 300th frame, the 454th frame, the 531th frame, the second row from left to write the 586th frame, the 633th frame, the 669th, the third row from left to write, the 700th frame, the 757th frame, the 800th frame. (STS-131 mission, Neptec Group)

In the case of complete occlusion, no methods that rely on image appearance can perform tracking. This dataset was also used to validate the performance of the suggested approach under occlusion. The $\mathrm{x}$ - and $\mathrm{y}$ - components of the detected feature points from the camera are shown in Figure. 3.31. As illustrated, the feature 

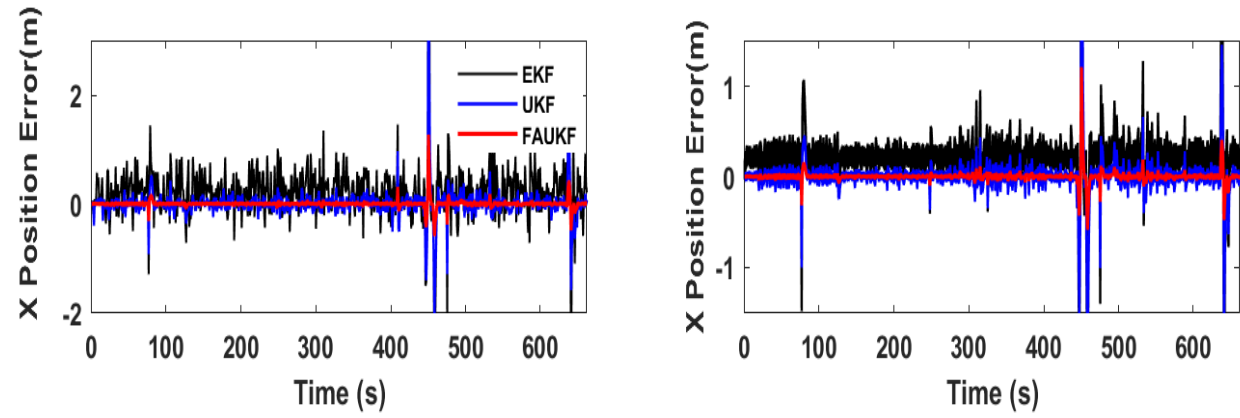

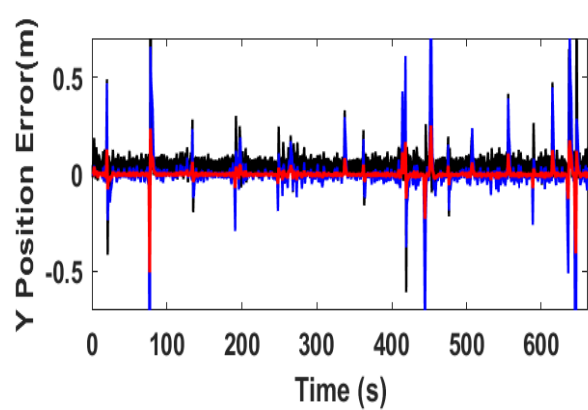

(a) Feature point 1 .
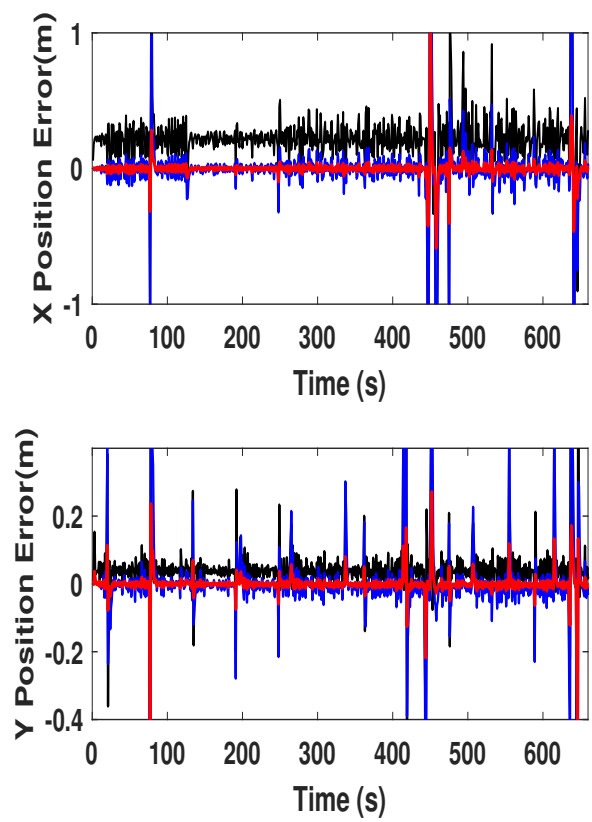

(c) Feature point 3.

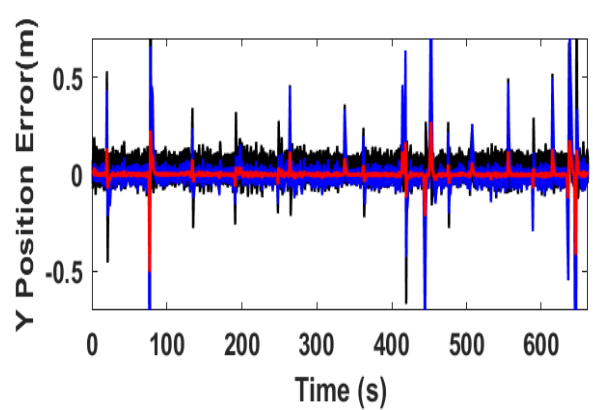

(b) Feature point 2.
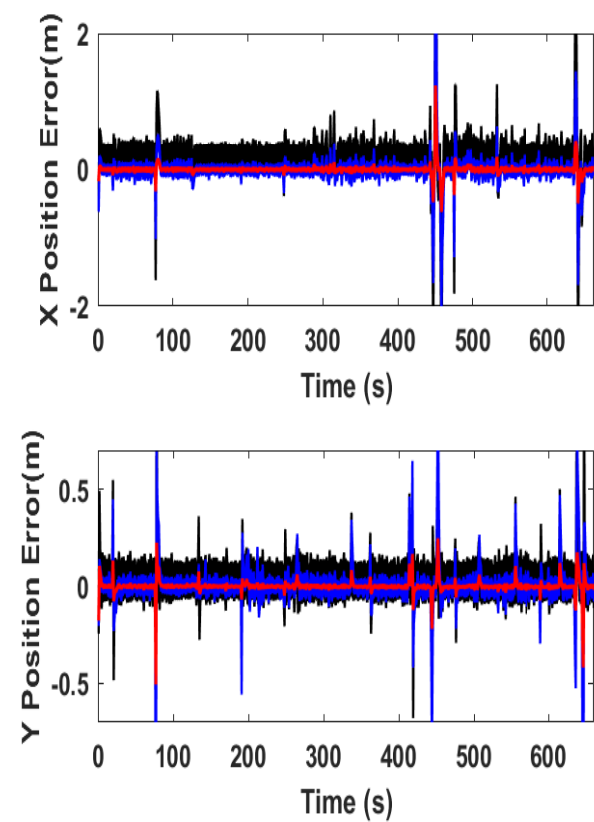

(d) Feature point 4 .

Figure. 3.30. Relative position errors of four feature points. 
point disappears in some frames due to motion. The actual and estimated positions of the feature point by FAUKF are shown in Figure. 3.32, and Figure. 3.33 provides the estimation performance of UKF, the estimated and actual position of the feature point. As shown, FAUKF outperformed the other filters and tracked moving feature points with higher accuracy, particularly when a feature point completely disappears at different times. The results also indicate that the FAUKF estimation error is less than that of the UKF. 

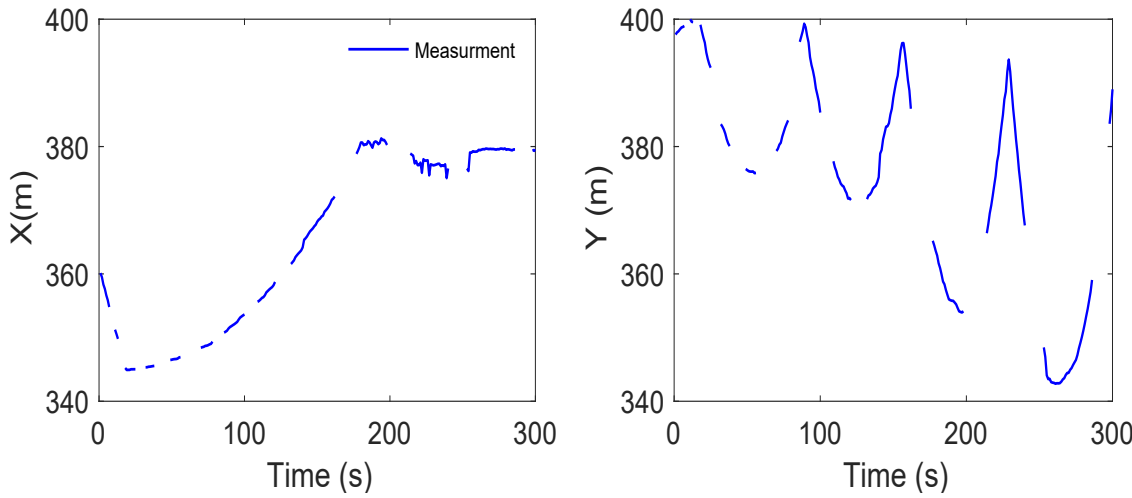

Figure. 3.31. Measurement data.
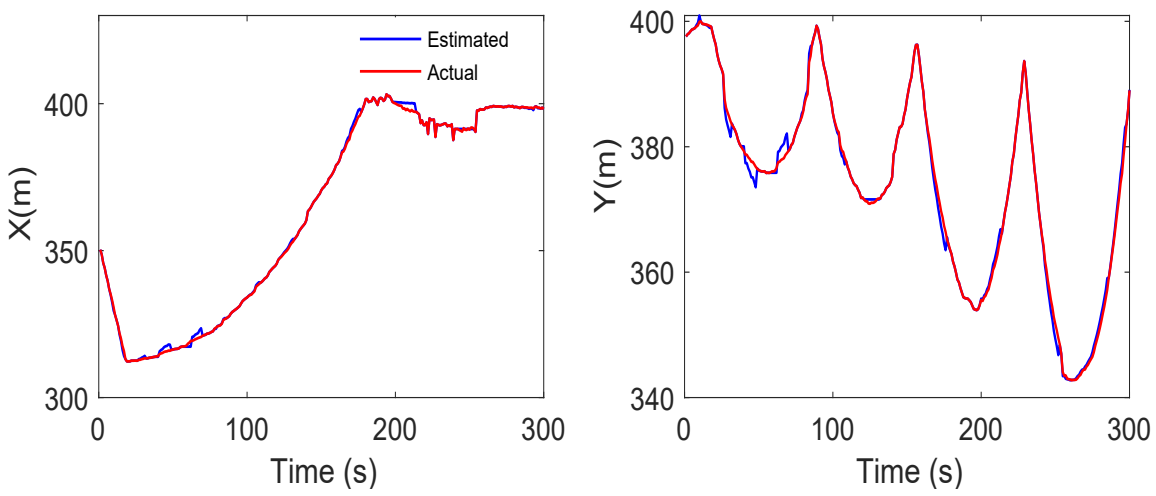

Figure. 3.32. FAUKF motion estimation.
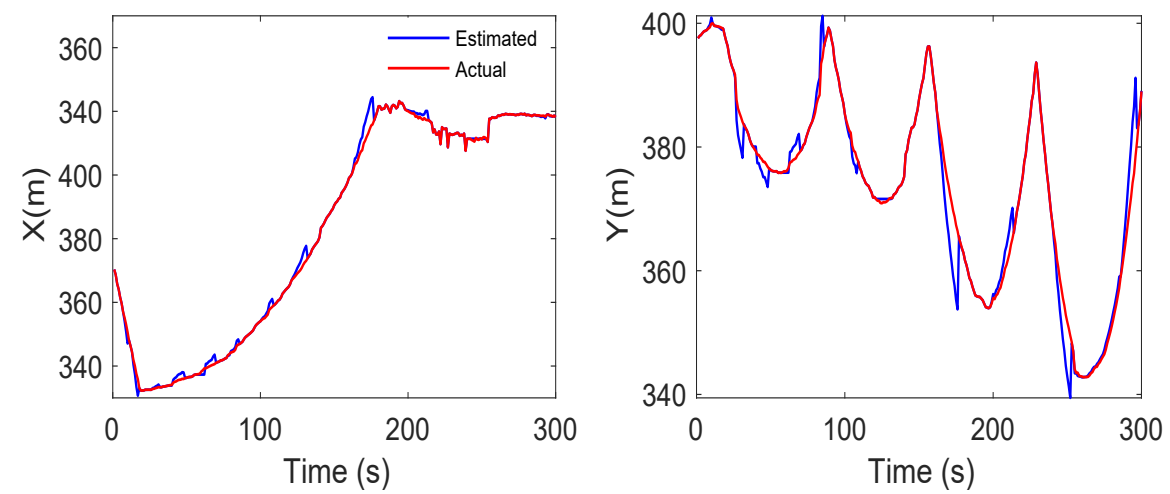

Figure. 3.33. UKF motion estimation. 


\section{Chapter 4}

\section{Space Target's Pose and Motion Estimation}

The structure of a space target, and estimates of its relative motion and pose, are the primary tasks of any formation flight, including missions to remove space debris. Acquiring these estimates for high-speed tumbling objects is challenging due to the lack of prior information about the target's structure and motion. This chapter of the thesis proposes a method to estimate the relative position, linear and angular velocities, attitude, and the structure of an uncooperative spacecraft based on visual navigation only. The visual system is a stereo camera that does not depend on markers attached to the target surface. The suggested approach employs UKF to perform the estimation procedure between two uncooperative spacecraft. The structure of the spacecraft was determined by estimating feature point positions. Besides, the relative attitude estimation is derived using Modified Rodrigues Parameters.

\subsection{Introduction}

Extensive research on the relative state estimation between space vehicles has been

conducted over the last decades. To obtain a full navigation solution, it is necessary 
to estimate the target's pose (position and orientation) and motion. This information is required for proper guidance, navigation and control of the chaser spacecraft. However, this information is usually unavailable, particularly in damaged satellites, space debris, and vibrating structures. Indeed, the relative navigation technology for uncooperative spacecraft is one of the key applications required for current and future space exploration. A non-cooperative target is not controlled, which means its attitude is in free motion in space. A chaser spacecraft must overcome these complications to establish accurate and reliable relative navigation. A visually based system for autonomous navigation is one of the best choices, but it is still a challenge for non-cooperative target spacecraft with no fiducial markers [201]. Of all visual systems, digital cameras are the optimal choice as the sensors can be employed for tracking and inspection in different ranges. Besides, digital cameras are smaller and lighter than active devices, and they use less power. Relative navigation technologies and the corresponding sensors for autonomous navigation have been studied and developed for several years. Many approaches have been proposed to determine the relative attitude and position between spacecraft, and different sensor types were chosen according to range and performance. The ETS-VII mission utilized a proximity camera to obtain the position of markers on the target for autonomous rendezvous and docking [202]. A near-field proximity operation sensor is known as the Advanced video guidance sensor was employed in the Orbital Express mission to obtain images of reflective markers for rendezvous and docking [203]. For autonomous rendezvous and docking with ISS, video meters and passive retro-reflectors are used to determine the relative pose [204]. However, these technologies cannot be implemented for space debris removal as space debris is not equipped with such artificial markers on its surface. NASA and the Canadian Space Agency (CSA) verified the TriDAR system for performing the relative navigation to an uncooperative and known target. TriDAR 
does not rely on any reference markers positioned on the target spacecraft. Instead, TriDAR relies on a laser-based 3D sensor and a thermal imager [195]. An algorithm based on the incremental smoothing and mapping for spacecraft proximity operation missions using a stereo vision was introduced by [125]. To test the algorithm, an experimental testbed known as Goggles was employed. A relative navigation strategy to determine the relative position and linear velocity on ISS Expedition 34 was validated with the SPHERES [205]. This approach is very challenging for large space targets, as it is based on the target's map creation. The relative pose estimation based on the Iterated Extended Kalman filter was proposed by Segal et al. [34]. The approach employed a stereoscopic vision system for tracking a set of feature points on the target spacecraft surface. In this work, the relative attitude estimation is derived using the quaternion. However, the quaternion's constraint creates a singularity in the Kalman filters covariance matrix [121]. A solution to simultaneously recover the three-dimensional-to-two-dimensional line and camera pose was introduced by Zhang et al. [206]. A method using the EKF integrated by ICP for a tumbling space target is proposed by Aghili et al. [207]. To determine the relative pose of the target object, the EKF used measurements from a mono camera system. However, this approach is suitable if prior knowledge of the geometry of the satellite and the initial pose are known, but without this information, the problem becomes more difficult. Furthermore, as large space targets are limited by the FOV, a monocular camera cannot supply enough information of the targets in close range [208]. A pose estimation algorithm for a large non-cooperative space target based on the two collaborating cameras was introduced by Du et al. [208]. This method relies on the detection of a rectangular feature on space targets. 


\subsection{Reference Frames}

A point's pose and motion in three-dimensional space must be specified with respect to a reference frame. Therefore, the precise definition of the reference frames to be used is fundamental to the navigation process. An appropriate explanation of the implemented coordinate systems is presented in this section. The target spacecraft is considered as an uncooperative space target, and the chaser is an inspecting spacecraft.

\subsubsection{Standard Earth-centered Inertial (ECI)}

$O_{I}-X_{I}, Y_{I}, Z_{I}:$ ECI frame is a global reference frame that has its origin at the center of the Earth. This reference frame does not rotate with Earth and serves as an inertial reference frame for satellites orbiting the Earth. Due to this, the ECI frame is used primarily in space applications. The $Z$ axis runs along the Earth's rotational axis pointing North, the $X$ axis points in the direction of the vernal equinox, and the $Y$ axis completes the right-handed orthogonal system.

\subsubsection{Orbital Reference Frame}

$O_{O}-X_{O}, Y_{O}, Z_{O}$ : The orbit reference frame defines the orientation of the orbital elements with respect to the central body. The origin of the orbital reference frame lies in the center of the mass of spacecraft. Where $X$ is in the direction of orbit motion, $Z$ is in the direction of the inertial coordinate origin, and $Y$ completes the right handed system. 


\subsubsection{Chaser Body-fixed Reference Frame}

$O_{L}-X_{L}, Y_{L}, Z_{L}$ : a local-vertical, local-horizontal Euler-Hill reference frame fixed on the center of mass of the chaser (leader) spacecraft and rotate with it. Where $X$ is a unit vector directed from the spacecraft outward, $Z$ normal to the chaser orbital plane, and $Y$ completes the setup.

\subsubsection{Stereo-vision-fixed Reference Frame}

$O_{S}-X_{S}, Y_{S}, Z_{S}$ : A Cartesian right-hand body reference frame attached to the right camera Center-of-Projection (CoP). $X$ is a unit vector directed from the right camera CoP toward the left camera CoP, $Z$ is vertical, positive upwards, and $Y$ completes the right-hand setup. The measurement information of the stereo camera is explained in this reference frame.

\subsubsection{Target Body-fixed Reference Frame}

$O_{T}-X_{T}, Y_{T}, Z_{T}$ : Is a cartesian right-hand body-fixed reference frame attached at the target's center of mass. With $X$ being a unit vector directed from the spacecraft outward, and the $Y$ axis is defined to point toward the Vernal equinox. The $Z$ axis completes the right-handed orthogonal reference frame.

\subsection{Estimation Methodology}

The estimation algorithm uses a set of feature points that are tracked by a stereo camera, and the coordinates of feature points are used as the input of the estimation process. The image processing, including feature detection, matching, tracking, and obtaining a depth map of the uncooperative target spacecraft is explained in the 
chapter 3.

\subsubsection{Dynamical Model}

Assume two rigid-body spacecraft are in orbits around the Earth, and the chaser spacecraft is equipped with vision sensors that capture $N$ feature points located on the other (target) spacecraft. These feature points can be represented as a vector, $\boldsymbol{F}^{i},(i=1,2, \ldots, N)$. Relative states were estimated using a sequence of stereo images. The relative nonlinear motion and observation model can be defined as:

$$
\begin{gathered}
\boldsymbol{x}_{k}=f\left(\boldsymbol{x}_{k-1}, u_{k}\right)+\boldsymbol{w}_{k} \\
\boldsymbol{y}_{k}=h\left(\boldsymbol{x}_{k}\right)+\boldsymbol{v}_{k}
\end{gathered}
$$

where

$$
\begin{aligned}
& \boldsymbol{w}_{k} \sim N\left(0, \boldsymbol{Q}_{k}\right) \\
& \boldsymbol{v}_{k} \sim N\left(0, \boldsymbol{R}_{k}\right)
\end{aligned}
$$

The vectors $\boldsymbol{x}_{k} \in \Re^{12+3}$ and $\boldsymbol{y}_{k} \in \Re^{4}$ represent the state of the system and measurement at a time instant $k$, respectively. $\boldsymbol{w}_{k}$ and $\boldsymbol{v}_{k}$ are the process and measurement noises.

\subsubsection{Process Model}

Using stereo images, the relative state is given by:

$$
\boldsymbol{x}_{k}=\left[\boldsymbol{r}_{C_{k}}, \dot{\boldsymbol{r}}_{C_{k}}, \boldsymbol{\omega}_{k}, \boldsymbol{\sigma}_{k}, \boldsymbol{F}_{k}^{i},\right]
$$

The state vector has three states for position: $\boldsymbol{r}_{C_{k}}=\left[x_{k}, y_{k}, z_{k}\right]^{T}$, three states 
for linear velocity $\dot{\boldsymbol{r}}_{C_{k}}=\left[\dot{x}_{k}, \dot{y}_{k}, \dot{z}_{k}\right]^{T}$, three states for angular velocity $\boldsymbol{\omega}_{k}=$ $\left[\omega_{x_{k}}, \omega_{y_{k}}, \omega_{z_{k}}\right]^{T}$ and three states for target orientation $\boldsymbol{\sigma}_{k}=\left[\sigma_{x_{k}}, \sigma_{y_{k}}, \sigma_{z_{k}}\right]^{T}$. As well, $3 N$ states for feature points' positions $\boldsymbol{F}_{k}^{i}=\left[F_{x_{k}}^{i}, F_{y_{k}}^{i}, F_{z_{k}}^{i}\right]^{T}$, where $i$ denotes the number of feature points.

\subsubsection{Relative Translational Dynamics}

The dynamic equations that represent the relative translational motion between two spacecrafts' centers of mass in chaser body-fixed reference frame can be described as [117]:

$$
\begin{gathered}
\ddot{x}=2 \dot{\vartheta}_{L} \dot{y}+\ddot{\vartheta}_{L} y+\dot{\vartheta}_{L}^{2} x-\frac{\mu\left(r_{L}+x\right)}{\left[\left(r_{L}+x\right)^{2}+y^{2}+z^{2}\right]^{1.5}}+\frac{\mu}{r_{L}} \\
\ddot{y}=-2 \dot{\vartheta}_{L} \dot{x}-\ddot{\vartheta}_{L} x+\dot{\vartheta}_{L}^{2} y-\frac{\mu(y)}{\left[\left(r_{L}+x\right)^{2}+y^{2}+z^{2}\right]^{1.5}} \\
\ddot{z}=-\frac{\mu(z)}{\left[\left(r_{L}+x\right)^{2}+y^{2}+z^{2}\right]^{1.5}}
\end{gathered}
$$

where $\mu$ is the Earth's gravitational constant and the chaser's orbital angular velocity, $\dot{\vartheta}_{L}$, and acceleration, $\ddot{\vartheta}_{L}$, are defined as:

$$
\begin{gathered}
\dot{\vartheta}_{L}=\sqrt{\frac{\mu}{a_{L}^{3}\left(1-e_{L}^{2}\right)^{3}}}\left(1+e_{L} \cos \vartheta_{L}\right)^{2} \\
\ddot{\vartheta}_{L}=\frac{-2 \dot{\vartheta}_{L} \dot{r}_{L}}{r_{L}}
\end{gathered}
$$

where, $\vartheta_{L}$ is the true anomaly of the chaser spacecraft, $a_{L}$ is the semi-major axis and $e_{L}$ is the eccentricity of the chaser spacecraft's orbit. Based on Newton's laws, the equations of motion for the chaser spacecraft in the absence of perturbing forces is given by: 


$$
\ddot{\boldsymbol{r}}_{L}=-\frac{\mu}{r_{L}^{3}} \boldsymbol{r}_{L}
$$

where $\boldsymbol{r}_{L} \in \Re^{3}$ indicates the chaser's center of mass position in ECI. The magnitudes of the position vector $\left\|\boldsymbol{r}_{L}\right\|_{2}$ can be written as:

$$
r_{L}=\frac{\left(1-e_{L}^{2}\right) a_{L}}{1+e_{L} \cos \vartheta_{L}}
$$

where $a_{L}$ is the semi-major axis of the chaser orbit and $e_{L}$ represents the chaser's orbit eccentricity.

\subsubsection{Relative Rotational Dynamics}

The purpose of the following development is to derive a model that describes the rotational motion of a target spacecraft in terms of relative MRPs and the angular velocity of the chaser. The quaternion representation is one of the most commonly used approaches for attitude estimation. However, it uses four components to represent the three degrees of freedom attitude motion, so quaternion components are dependent, which leads to a constraint that they must have a unit norm. The constraint creates a singularity in the Kalman filter covariance matrix [121]. One approach to this shortcoming is using MRPs. A set of three attitude MRPs can be obtained from the quaternion as follows:

$$
\boldsymbol{\sigma}=\frac{1}{1+q_{4}}\left[\begin{array}{l}
q_{1} \\
q_{2} \\
q_{3}
\end{array}\right]
$$

The relative attitude kinematics from the chaser spacecraft to the target can be 
defined as follows [121]:

$$
\dot{\boldsymbol{\sigma}}=\frac{1}{2}\left(\left(\frac{1-\boldsymbol{\sigma}^{T} \boldsymbol{\sigma}}{2}\right) \boldsymbol{I}_{3 \times 3}+\left[\boldsymbol{\sigma}^{\times}\right]+\boldsymbol{\sigma} \boldsymbol{\sigma}^{T}\right) \boldsymbol{\omega}
$$

where $\boldsymbol{I}_{3 \times 3}$ is a unit matrix, $\boldsymbol{\omega}$ is defined as the rotation rates of the target relative to the chaser spacecraft resolved in the target coordinate frame. The relative angular velocity in the chaser coordinate frame can be express as:

$$
\left.\omega\right|_{L}=\left.\boldsymbol{D}(\boldsymbol{\sigma}) \omega\right|_{T}
$$

The rotation matrix, $\boldsymbol{D}(\boldsymbol{\sigma})$ which transform from the target to the chaser coordinate frames in terms of the MRPs, is expressed as:

$$
\boldsymbol{D}(\boldsymbol{\sigma})=\boldsymbol{I}_{3 \times 3}-\frac{4\left(1-\boldsymbol{\sigma}^{T} \boldsymbol{\sigma}\right)}{\left(1+\boldsymbol{\sigma}^{T} \boldsymbol{\sigma}\right)^{2}}\left[\boldsymbol{\sigma}^{\times}\right]+\frac{8}{\left(1+\boldsymbol{\sigma}^{T} \boldsymbol{\sigma}\right)^{2}}\left[\boldsymbol{\sigma}^{\times}\right]^{2}
$$

where

$$
\left[\boldsymbol{\sigma}^{\times}\right]=\left[\begin{array}{ccc}
0 & -\sigma_{3} & \sigma_{2} \\
\sigma_{3} & 0 & -\sigma_{1} \\
-\sigma_{2} & \sigma_{1} & 0
\end{array}\right]
$$

The MRPs are valid for angle errors that are less than a full rotation. This is adequate, as the quaternion errors are reset to zero after every measurement update. Thus, the quaternion can be recovered from the MRPs using the quaternion product of the previous estimate and the error quaternion.

$$
\boldsymbol{q}_{k}=\delta \boldsymbol{q} \otimes \boldsymbol{q}_{k-1}
$$

The angular velocity of the target relative to the chaser spacecraft is denoted as: 


$$
\omega \triangleq \omega_{T}-\omega_{L}
$$

where $\boldsymbol{\omega}_{T}$ and $\boldsymbol{\omega}_{L}$ are the angular velocities of the target and chaser spacecraft, respectively. The differentiation of Equation (4.19) with respect to the chaser spacecraft is given by:

$$
\dot{\omega}=\dot{\omega}_{T}-\dot{\omega}_{L}
$$

The Equation (4.20) in the chaser frame can be rewritten as [34]:

$$
\dot{\boldsymbol{\omega}}=\boldsymbol{D}(\boldsymbol{\sigma}) \dot{\boldsymbol{\omega}}_{T}-\dot{\boldsymbol{\omega}}_{L}-\boldsymbol{\omega}_{L} \boldsymbol{\omega}
$$

Multiplying Equation (4.21) by the inertia tensor of the chaser $\boldsymbol{I}_{L}$ gives:

$$
\boldsymbol{I}_{L} \dot{\boldsymbol{\omega}}=\boldsymbol{I}_{L} \boldsymbol{D}(\boldsymbol{\sigma}) \dot{\boldsymbol{\omega}}_{T}-\boldsymbol{I}_{L} \dot{\boldsymbol{\omega}}_{L}-\boldsymbol{I}_{L} \hat{\boldsymbol{\omega}}_{L} \boldsymbol{\omega}
$$

An external torque on the body of the chaser and target are defined as:

$$
\begin{aligned}
& \boldsymbol{N}_{L}=\boldsymbol{I}_{L} \dot{\boldsymbol{\omega}}_{L}+\boldsymbol{\omega}_{L} \times \boldsymbol{I}_{L} \boldsymbol{\omega}_{L} \\
& \boldsymbol{N}_{T}=\boldsymbol{I}_{T} \dot{\boldsymbol{\omega}}_{T}+\boldsymbol{\omega}_{T} \times \boldsymbol{I}_{T} \boldsymbol{\omega}_{T}
\end{aligned}
$$

By substituting Equations (4.23) and (4.24) into Equation (4.22) we have:

$$
\begin{gathered}
\boldsymbol{I}_{L} \dot{\boldsymbol{\omega}}=\boldsymbol{I}_{L} \boldsymbol{D}(\boldsymbol{\sigma}) \boldsymbol{I}_{T}^{-1}\left[\boldsymbol{N}_{T}-\boldsymbol{D}^{T}(\boldsymbol{\sigma})\left(\boldsymbol{\omega}+\boldsymbol{\omega}_{L}\right) \times \boldsymbol{I}_{T} \boldsymbol{D}^{T}(\boldsymbol{\sigma})^{T}\left(\boldsymbol{\omega}+\boldsymbol{\omega}_{L}\right)\right] \\
-\boldsymbol{I}_{L} \boldsymbol{\omega}_{L} \times \boldsymbol{\omega}-\left[\boldsymbol{N}_{L}-\left(\boldsymbol{\omega}_{L} \times \boldsymbol{I}_{L} \boldsymbol{\omega}_{L}\right)\right]
\end{gathered}
$$


For the torque-free motion of a target, $N_{T}=0$. Therefore equation 4.26 can be rewritten as:

$$
\begin{gathered}
\boldsymbol{I}_{L} \dot{\boldsymbol{\omega}}=\boldsymbol{I}_{L} \boldsymbol{D}(\boldsymbol{\sigma}) \boldsymbol{I}_{T}^{-1}\left[\boldsymbol{D}^{T}(\boldsymbol{\sigma})\left(\boldsymbol{\omega}+\boldsymbol{\omega}_{L}\right) \times \boldsymbol{I}_{T} \boldsymbol{D}^{T}(\boldsymbol{\sigma})^{T}\left(\boldsymbol{\omega}+\boldsymbol{\omega}_{L}\right)\right] \\
-\boldsymbol{I}_{L} \boldsymbol{\omega}_{L} \times \boldsymbol{\omega}-\left[\boldsymbol{N}_{L}-\left(\boldsymbol{\omega}_{L} \times \boldsymbol{I}_{L} \boldsymbol{\omega}_{L}\right)\right]
\end{gathered}
$$

\subsubsection{Kinematically Coupled Spacecraft Relative Motion Model}

When a feature point is observed by the stereo camera, the vector from the observed feature point and the camera center of projection, as shown in Figure. 4.1, can be given by:

$$
\boldsymbol{r}_{C_{i}}=\boldsymbol{r}_{C_{0}}+\boldsymbol{F}^{i}-\boldsymbol{T}_{C / L}
$$

where $\boldsymbol{r}_{C_{0}}$ represents the relative position from the chaser center of mass to the target center of mass, expressed in the orbital frame. $\boldsymbol{T}_{C / L}=\left[\boldsymbol{T}_{x_{C / L}}, \boldsymbol{T}_{y_{C / L}}, \boldsymbol{T}_{z_{C / L}}\right]^{T}$ is a vector directed from the origin of the chaser coordinate system to the right camera center

of projection. $\boldsymbol{F}^{i}=\left[F_{x}^{i}, F_{y}^{i}, F_{z}^{i}\right]^{T}$ is a vector directed from the origin of the space target to an arbitrary point on the target spacecraft that was captured by the stereo camera. In the special case where the origin of the chaser spacecraft coincides with the camera center $\boldsymbol{F}^{i}=[0,0,0]^{T}$. The first and second time derivative of Equation (4.27) in a local-vertical, local-horizontal Euler-Hill reference frame fixed to the chaser spacecraft's center of mass can be written as:

$$
\dot{\boldsymbol{r}}_{C_{i}}=\dot{\boldsymbol{r}}_{C_{0}}+\boldsymbol{\omega} \times \boldsymbol{F}^{i}
$$




$$
\ddot{\boldsymbol{r}}_{C_{i}}=\ddot{\boldsymbol{r}}_{C_{0}}+\dot{\boldsymbol{\omega}} \times \boldsymbol{F}^{i}+\boldsymbol{\omega} \times\left(\boldsymbol{\omega} \times \boldsymbol{F}^{i}\right)
$$

Considering the target as a rigid body, all points on it have the same velocity and acceleration. Thus, substituting Equations (4.28) and (4.29) into the Equation (4.8) yields the following general description of the translational motion between any arbitrary points on the target and the camera center of the projection in the absence of perturbing forces:

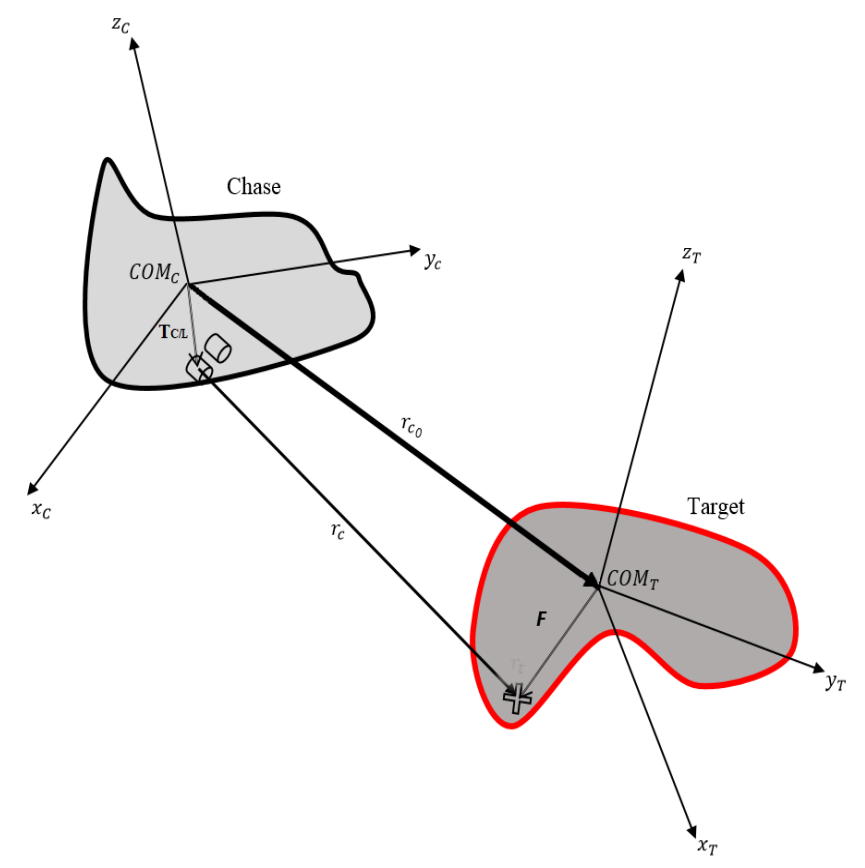

Figure. 4.1. Two rigid-body spacecraft reference frames.

$$
\begin{gathered}
\ddot{x}_{i}=\left[\omega_{y}\left(\omega_{x} F_{y}^{i}-\omega_{y} F_{x}^{i}\right)-\omega_{z}\left(\omega_{z} F_{x}^{i}-\omega_{x} F_{z}^{i}\right)\right]+\dot{\omega}_{y} F_{z}^{i}-\dot{\omega}_{z} F_{y}^{i} \\
+2 \dot{\vartheta}_{L}\left[\dot{y}_{i}-\left(\omega_{z} F_{x}^{i}+\omega_{x} F_{z}^{i}\right)\right]-\ddot{\vartheta}_{L}\left(y_{i}-F_{y}^{i}+T_{y C / L}\right)+\dot{\vartheta}_{L}^{2}\left(x_{i}-F_{x}^{i}+T_{x C / L}\right) \\
+\frac{-\mu\left(r_{L}+x_{i}-F_{x}^{i}+T_{x C / L}\right)}{\left[\left(r_{L}+x_{i}-F_{x}^{i}+T_{x C / L}\right)^{2}+\left(y_{i}-F_{y}^{i}+T_{y C / L}\right)^{2}+\left(z_{i}-F_{z}^{i}+T_{z C / L}\right)^{2}\right] \frac{3}{2}+\frac{\mu}{r_{L}^{2}}}
\end{gathered}
$$




$$
\begin{gathered}
\ddot{y}_{i}=\left[\omega_{z}\left(\omega_{y} F_{z}^{i}-\omega_{z} F_{y}^{i}\right)+\omega_{x}\left(\omega_{x} F_{y}^{i}-\omega_{y} F_{x}^{i}\right)\right]+\dot{\omega}_{z} F_{x}^{i}-\dot{\omega}_{x} F_{z}^{i} \\
+2 \dot{\vartheta}_{L}\left[\dot{x}_{i}-\left(\omega_{y} F_{z}^{i}+\omega_{z} F_{y}^{i}\right)\right]+\dot{\vartheta}_{L}^{2}\left(y_{i}-F_{y}^{i}+T_{y C / L}\right)-\ddot{\vartheta}_{L}\left(x_{i}-F_{x}^{i}+T_{x C / L}\right) \\
+\mu\left(y_{i}-F_{y}^{i}+T_{y C / L}\right) \\
{\left[\left(r_{L}+x_{i}-F_{x}^{i}+T_{x C / L}\right)^{2}+\left(y_{i}-F_{y}^{i}+T_{y C / L}\right)^{2}+\left(z_{i}-F_{z}^{i}+T_{z C / L}\right)^{2}\right]^{\frac{3}{2}}} \\
+\frac{-\mu\left(z_{i}-F_{z}^{i}+T_{z C / L}\right)}{\left[\left(r_{L}+x_{i}-F_{x}^{i}+T_{x C / L}\right)^{2}+\left(y_{i}-F_{y}^{i}+T_{y C / L}\right)^{2}+\left(z_{i}-F_{z}^{i}+T_{z C / L}\right)^{2}\right]^{\frac{3}{2}}}
\end{gathered}
$$

\section{Translational - rotational model}

The state variables for complete translational-rotational model are defined as follows:

$$
\boldsymbol{x}_{i}=\left[\boldsymbol{r}_{C i}^{T}, \dot{\boldsymbol{r}}_{C i}^{T}, \boldsymbol{\omega}^{T}, \boldsymbol{\sigma}^{T}, \boldsymbol{F} \boldsymbol{i}^{T}\right]^{T}
$$

The nonlinear dynamic equation is expressed as:

$$
\dot{\boldsymbol{x}}_{i}=\boldsymbol{f}\left(x_{i}\right)+\boldsymbol{w}
$$

where $\boldsymbol{w}$ is assumed to be a white Gaussian process noise with covariance matrix $\boldsymbol{Q}_{k}$ which represents the model uncertainty, and $f(x)$ can be rewritten as:

$$
\begin{gathered}
\boldsymbol{f}(\boldsymbol{x})=\left[f_{1}, f_{2}, f_{3}, \ldots, f_{15}\right] \\
f_{1}=x_{4} \\
f_{2}=x_{5}
\end{gathered}
$$




$$
f_{3}=x_{6}
$$

$$
\begin{aligned}
& f_{4}=\left[x_{8}\left(x_{7} x_{14}+x_{8} x_{13}\right)-x_{9}\left(x_{9} x_{13}-x_{7} x_{15}\right)\right]+\dot{x}_{8} x_{15}-\dot{x}_{9} x_{14} \\
& 2 \dot{\vartheta}_{L}\left[x_{5}-\left(x_{9} x_{13}+x_{7} x_{15}\right)\right]-\ddot{\vartheta}_{L}\left(x_{2}-x_{14}+T_{y C / L}\right)+\dot{\vartheta}_{L}^{2}\left(x_{1}-x_{13}+T_{x C / L}\right)+ \\
& -\mu\left(r_{L}+x_{1}-x_{13}+T_{x C / L}\right) \\
& {\left[\left(r_{L}+x_{1}-x_{13}+T_{x C / L}\right)^{2}+\left(x_{2}-x_{14}+T_{y C / L}\right)^{2}+\left(x_{3}-x_{15}+T_{z C / L}\right)^{2}\right]^{\overline{2}}} \\
& \frac{\mu}{3}+\frac{\mu}{r_{L}^{2}} \\
& f_{5}=\left[x_{9}\left(x_{8} x_{15}+x_{9} x_{14}\right)+x_{7}\left(x_{7} x_{14}-x_{8} x_{13}\right)\right]+\dot{x}_{9} x_{13}-\dot{x}_{7} x_{15} \\
& -2 \dot{\vartheta}_{L}\left[x_{4}-\left(x_{7} x_{15}+x_{9} x_{14}\right)\right]-\ddot{\vartheta}_{L}\left(x_{1}-x_{13}+T_{x C / L}\right)-\dot{\vartheta}_{L}^{2}\left(x_{2}-x_{14}+T_{y C / L}\right)+ \\
& -\mu\left(x_{2}-x_{14}+T_{y C / L}\right) \\
& {\left[\left(r_{L}+x_{1}-x_{13}+T_{x C / L}\right)^{2}+\left(x_{2}-x_{14}+T_{y C / L}\right)^{2}+\left(x_{3}-x_{15}+T_{z C / L}\right)^{2}\right]^{\overline{2}}} \\
& f_{6}=\left[x_{7}\left(x_{9} x_{13}-x_{7} x_{15}\right)+x_{8}\left(x_{8} x_{15}-x_{9} x_{14}\right)\right]-\dot{x}_{7} x_{14}+\dot{x}_{8} x_{13} \\
& +\frac{-\mu\left(x_{3}-x_{15}+T_{z C / L}\right)}{3} \\
& {\left[\left(r_{L}+x_{1}-x_{13}+T_{x C / L}\right)^{2}+\left(x_{2}-x_{y 14}+T_{y C / L}\right)^{2}+\left(x_{3}-x_{15}+T_{z C / L}\right)^{2}\right]^{\overline{2}}}
\end{aligned}
$$

$$
\left[\begin{array}{c}
f_{7} \\
f_{8} \\
f_{9}
\end{array}\right]=\boldsymbol{D}\left(\boldsymbol{X}_{\sigma}\right) \boldsymbol{I}_{T}^{-1}\left[-\boldsymbol{D}\left(\boldsymbol{X}_{\sigma}\right)\left(\boldsymbol{X}_{\omega}+\boldsymbol{\omega}_{L}\right) \times \boldsymbol{I}_{T} \boldsymbol{D}\left(\boldsymbol{X}_{\sigma}\right)^{T}\left(\boldsymbol{X}_{\omega}+\boldsymbol{\omega}_{L}\right)\right]
$$




$$
\begin{gathered}
{\left[\begin{array}{c}
f_{10} \\
f_{11} \\
f_{12}
\end{array}\right]=\frac{1}{2}\left(\left(\frac{1-\boldsymbol{X}_{\sigma}^{T} \boldsymbol{X}_{\sigma}}{2}\right) \boldsymbol{I}_{3 \times 3}\left[\boldsymbol{X}_{\sigma}^{\times}\right]+\boldsymbol{X}_{\sigma} \boldsymbol{X}_{\sigma}^{T}\right) \boldsymbol{D}\left(\boldsymbol{X}_{\sigma}\right)\left[\begin{array}{l}
x_{7} \\
x_{8} \\
x_{9}
\end{array}\right]} \\
{\left[\begin{array}{c}
f_{13} \\
f_{14} \\
f_{15}
\end{array}\right]=\boldsymbol{X}_{\boldsymbol{\omega}} \times \boldsymbol{X}_{\boldsymbol{F}}}
\end{gathered}
$$

where $\boldsymbol{X}_{\boldsymbol{\sigma}}=\left[x_{10}, x_{11}, x_{12}\right]^{T}, \boldsymbol{X}_{\boldsymbol{\omega}}=\left[x_{10}, x_{11}, x_{12}\right]^{T}, \boldsymbol{X}_{\boldsymbol{F}}=\left[x_{13}, x_{14}, x_{15}\right]^{T}$

\subsubsection{Observation Model}

The stereo rig is made up of two parallel cameras mounted on the chaser spacecraft and pointed at the target, with the left and right camera center of projection separated by the baseline, as shown in Figure. 4.2. The line-of-sight between the observed feature points and the right camera's center of projection $\boldsymbol{r}_{C_{i_{k}}}=\left[x_{i_{k}}, y_{i_{k}}, z_{i_{k}}\right]$ from Figure. 4.1 can be written as:

$$
\boldsymbol{r}_{C_{i_{k}}}=\boldsymbol{r}_{C_{0}}+\boldsymbol{D}(q) \boldsymbol{F}_{k}^{i} \quad \text { for } \quad i=1,2, \ldots, N
$$

where $\boldsymbol{F}_{k}^{i} \in \Re^{3}$ is the feature point location relative to the target's center of mass, expressed in the target's coordinate system. When the stereo rig observes feature points, a perspective projection model can formulate the visual observations. The perspective projection that transforms the feature points on the image plane can be written as: 


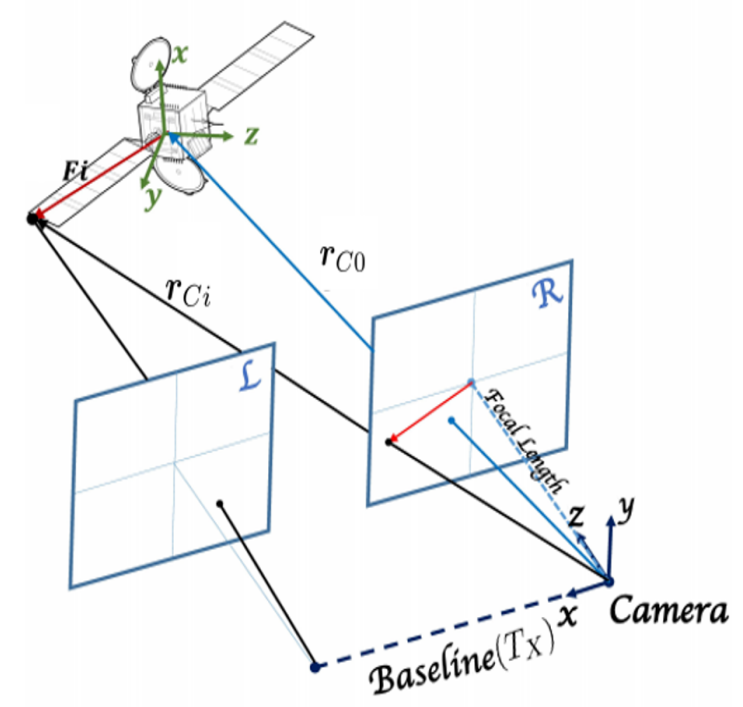

Figure. 4.2. The stereo rig system.

$$
\begin{aligned}
& U_{r i}=f_{c} \frac{x_{i_{k}}}{z_{i_{k}}} \\
& V_{r i}=f_{c} \frac{y_{i_{k}}}{z_{i_{k}}}
\end{aligned}
$$

Similarly, for the left camera we have:

$$
\begin{gathered}
U_{l i}=f_{c} \frac{x_{i_{k}}+T_{X}}{z_{i_{k}}} \\
V_{l i}=f_{c} \frac{y_{i_{k}}}{z_{i_{k}}}
\end{gathered}
$$

From Equations (4.46) and (4.47), the observation equation can be written as:

$$
\boldsymbol{y}_{i_{k}}=\boldsymbol{h}_{i}\left(\boldsymbol{x}_{k}\right)+\boldsymbol{v}_{i_{k}}, \quad i=1,2, \ldots, N
$$

where

where $T_{X}$ is the camera baseline and $\boldsymbol{v}_{i_{k}}$ is assumed to be a zero-mean white noise. 


\subsection{Algorithm Summary}

The algorithm developed for the state vector estimation of an uncooperative target in space is summarized in Table 4.1.

Table 4.1: Target pose and motion estimation using UKF.

Sequential estimation with the Unscented Kalman Filter

1. for $i=1$ :All feature points do

2. Initialization: $\hat{\mathbf{x}}_{0}=E\left(\mathbf{x}_{0}\right), \quad \mathbf{P}_{0}=E\left[\left(\mathbf{x}_{0}-\hat{\mathbf{x}}_{0}\right)\left(\mathbf{x}_{0}-\hat{\mathbf{x}}_{0}\right)^{T} \quad \lambda=\sigma^{2}(L+\kappa)-L\right.$

3. for $k=1: N$ do

4. $\quad$ Compute sigma points: $\chi_{k-1}=\left[\begin{array}{ll}\hat{\mathbf{x}}_{k-1} & \left.\hat{\mathbf{x}}_{k-1} \pm \sqrt{(L+\lambda}\right) \sqrt{\mathbf{P}_{k-1}}\end{array}\right]$

5. Obtain previous step state: $\boldsymbol{\chi}_{k \mid k-1}^{(i)}=\boldsymbol{f}\left(\boldsymbol{\chi}_{k-1}, \boldsymbol{u}_{k}\right)$

6. $\quad$ Propagate the mean: $\hat{\boldsymbol{x}}_{k \mid k-1}=\sum_{i=0}^{2 L} \eta_{i}^{(m)} \chi_{k \mid k-1}^{(i)}$

7. Propagate the covariance:

$\boldsymbol{P}_{k \mid k-1}=\boldsymbol{Q}+\sum_{i=0}^{2 L} \eta_{i}^{(c)}\left(\boldsymbol{\chi}_{k \mid k-1}^{(i)}-\hat{\boldsymbol{x}}_{k \mid k-1}\right)\left(\boldsymbol{\chi}_{k \mid k-1}^{(i)}-\hat{\boldsymbol{x}}_{k \mid k-1}\right)^{T}$

8. Obtain the current step measurement $\mathbf{y}_{k}$ from the stereo camera:

$$
\hat{\boldsymbol{z}}_{k \mid k-1}=\sum_{i=0}^{2 L} \eta_{i}^{(m)} \gamma_{k \mid k-1}^{(i)}, \quad \boldsymbol{\gamma}_{k \mid k-1}^{(i)}=\boldsymbol{h}\left(\boldsymbol{\chi}_{k \mid k-1}^{(i)}\right)
$$

9. Compute the covariance matrix:

$$
\mathbf{P}_{k}^{z z}=\sum_{i=0}^{2 L} \eta_{i}^{(c)}\left(\boldsymbol{\gamma}_{k \mid k-1}^{(i)}-\hat{\boldsymbol{y}}_{k \mid k-1}\right)\left(\boldsymbol{\gamma}_{k \mid k-1}^{(i)}-\hat{\boldsymbol{y}}_{k \mid k-1}\right)^{T}
$$

10. Compute the cross-covariance matrix:

$$
\mathbf{P}_{k}^{x z}=\sum_{i=0}^{2 L} \eta_{i}^{(c)}\left(\boldsymbol{\chi}_{k \mid k-1}^{(i)}-\hat{\boldsymbol{x}}_{k \mid k-1}\right)\left(\boldsymbol{\gamma}_{k \mid k-1}^{(i)}-\hat{\boldsymbol{z}}_{k \mid k-1}\right)^{T}
$$

11. Compute the Kalman Gain: $\boldsymbol{K}_{k}=\boldsymbol{P}_{k}^{x z}\left(\mathbf{P}_{k}^{z z}\right)^{-1}$

12. Compute the updated state estimate: $\hat{\boldsymbol{x}}_{k}=\hat{\boldsymbol{x}}_{k \mid k-1}+\boldsymbol{K}_{k}\left(\boldsymbol{y}_{k}-\hat{\boldsymbol{y}}_{k \mid k-1}\right)$

13. Update the covariance matrix $\mathbf{P}_{k}=\mathbf{P}_{k \mid k-1}-\mathbf{K}_{k} \mathbf{P}_{k}^{z z} \boldsymbol{K}_{k}^{T}$

14. end for

15. end for 
Table 4.2: States' initial values.

\begin{tabular}{lr}
\hline \hline \multicolumn{1}{c}{ Initial values } & \\
\cline { 1 - 1 } Parameter Names & \\
\hline Initial relative position & $\boldsymbol{r}_{C_{i}}(0)=[20,50,200]^{T} \mathrm{~m}$ \\
Initial relative linear velocity & $\dot{\boldsymbol{r}}_{C_{i}}(0)=[0,0.2,0]^{T} \mathrm{~m} / \mathrm{s}$ \\
Initial relative angular velocity & $\boldsymbol{\omega}(0)=[0,0,0]^{T} \mathrm{rad} / \mathrm{s}$ \\
Initial quaternion & $\boldsymbol{q}(0)=[0,0,0,1]^{T} \mathrm{rad}$ \\
Inertia tensors & $\boldsymbol{I}=\operatorname{diag}(400,500,500) \mathrm{kgm}^{2}$ \\
\hline \hline
\end{tabular}

\subsection{Simulation Experiment}

The simulation experiment is performed to validate the performance of the suggested algorithms for pose and motion estimation between non-cooperative spacecraft. It is assumed that both spacecraft are in low earth orbit and the chaser spacecraft is in a circular orbit with eccentricity $e_{L}=0$, semi-major axis $a_{L}=7170 \mathrm{~km}$, inclination $i_{L}$ $=10 \mathrm{deg}$, argument of the perigee $\omega=300 \mathrm{deg}$, right ascension of ascending node $\Omega$ $=0 \mathrm{deg}$ and the initial true anomaly is $20 \mathrm{deg}$. The masses of the chaser and target are $m_{L}=m_{F}=410 \mathrm{~kg}$. The relative initial conditions between the chaser and target are given in Table 4.2, and the standard reference frames are shown in Figure. 4.3.

The target spacecraft is assumed to be in a torque-free tumbling motion. A baseline of one meter separates the two cameras on the chaser spacecraft, and they capture the images of feature points on the target spacecraft. It is also assumed that four feature points are detected and tracked during the simulation. Typically, more than four feature points can be detected in an experimental case unless the target is either too bright or not adequately illuminated. With this assumption, filter performance can be tested when imperfect measurements are available. The 


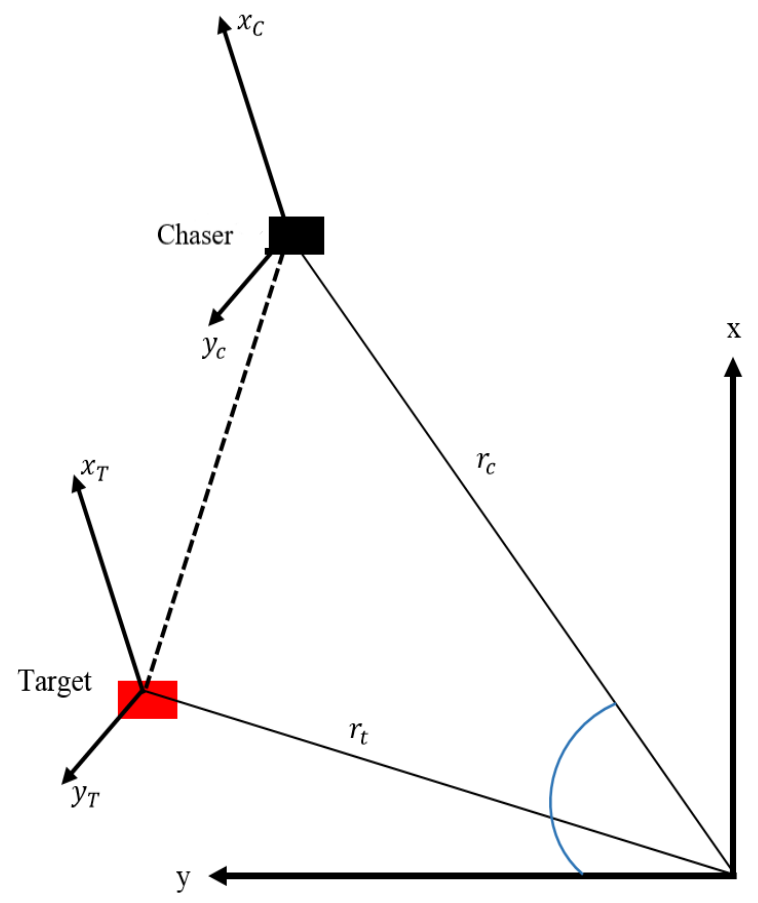

Figure. 4.3. Reference and body frames.

specifications of the feature points are shown in Table 4.3.

Table 4.3: Feature point specifications in the simulation.

\begin{tabular}{cccc}
\hline \hline Feature Point Number & $\mathrm{x}(\mathrm{m})$ & $\mathrm{y}(\mathrm{m})$ & $\mathrm{z}(\mathrm{m})$ \\
\hline 1 & 2 & 1 & -1 \\
2 & 0.5 & -1 & 1 \\
3 & 0 & 2 & -1 \\
4 & 1 & 1 & -1 \\
\hline \hline
\end{tabular}

The feature points' estimation error defined as:

$$
e_{p_{F i}}=\sqrt{\left(F_{x}^{i}-\hat{F}_{x}^{i}\right)^{2}+\left(F_{y}^{i}-\hat{F}_{y}^{i}\right)^{2}+\left(F_{z}^{i}-\hat{F}_{z}^{i}\right)^{2}}
$$




$$
e_{p_{F}}=\sum_{i=1}^{N} e_{p_{F i}}
$$

where, $\boldsymbol{F}^{i}=\left[F_{x}^{i}, F_{y}^{i}, F_{z}^{i}\right]^{T}$ denotes the $i^{t h}$ feature point position. The process covariance matrix was defied as:

$$
\boldsymbol{Q}=\left[\begin{array}{ccccc}
\delta_{r_{C}}^{2} I_{3 \times 3} & 0_{3 \times 3} & 0_{3 \times 3} & 0_{3 \times 3} & 0_{3 \times 3} \\
0_{3 \times 3} & \delta_{\dot{r}_{C}}^{2} I_{3 \times 3} & 0_{3 \times 3} & 0_{3 \times 3} & 0_{3 \times 3} \\
0_{3 \times 3} & 0_{3 \times 3} & \delta_{\sigma}^{2} I_{3 \times 3} & 0_{3 \times 3} & 0_{3 \times 3} \\
0_{3 \times 3} & 0_{3 \times 3} & 0_{3 \times 3} & \delta_{\omega}^{2} I_{3 \times 3} & 0_{3 \times 3} \\
0_{3 \times 3} & 0_{3 \times 3} & 0_{3 \times 3} & 0_{3 \times 3} & \delta_{F^{i}}^{2} I_{3 \times 3}
\end{array}\right]
$$

where, $\delta_{r_{C}}^{2}=0.0001 \mathrm{~m}^{2}, \delta_{\dot{r}_{C}}^{2}=0.0001 \mathrm{~m}^{2} / \mathrm{s}^{2}, \delta_{\sigma}^{2}=0.0001 \mathrm{rad}, \delta_{\omega}^{2}=0.0001 \mathrm{rad}^{2} / \mathrm{s}^{2}$, $\delta_{F^{i}}^{2}=0.0001 \mathrm{~m}^{2}$

Noisy observations were modeled by adding noise to the perspective projection of the simulated trajectories of each one of the feature points. The observation noise was assumed to be zero-mean Gaussian with a standard deviation of $10^{-5}$.

$$
\boldsymbol{R}=10^{-5} \boldsymbol{I}_{4 N}
$$

Note that these initial uncertainty values and conditions are based on examples in relevant literature and experiments [34] to have the best set to allow the simulation model presents the result close to real conditions. Of course increase or decreasing uncertainty in the system can increase or decrease the estimation errors, respectively.

Other parameters of UKF are defined as $\kappa=0, \beta=2$, and $\alpha=10^{-3}$. 

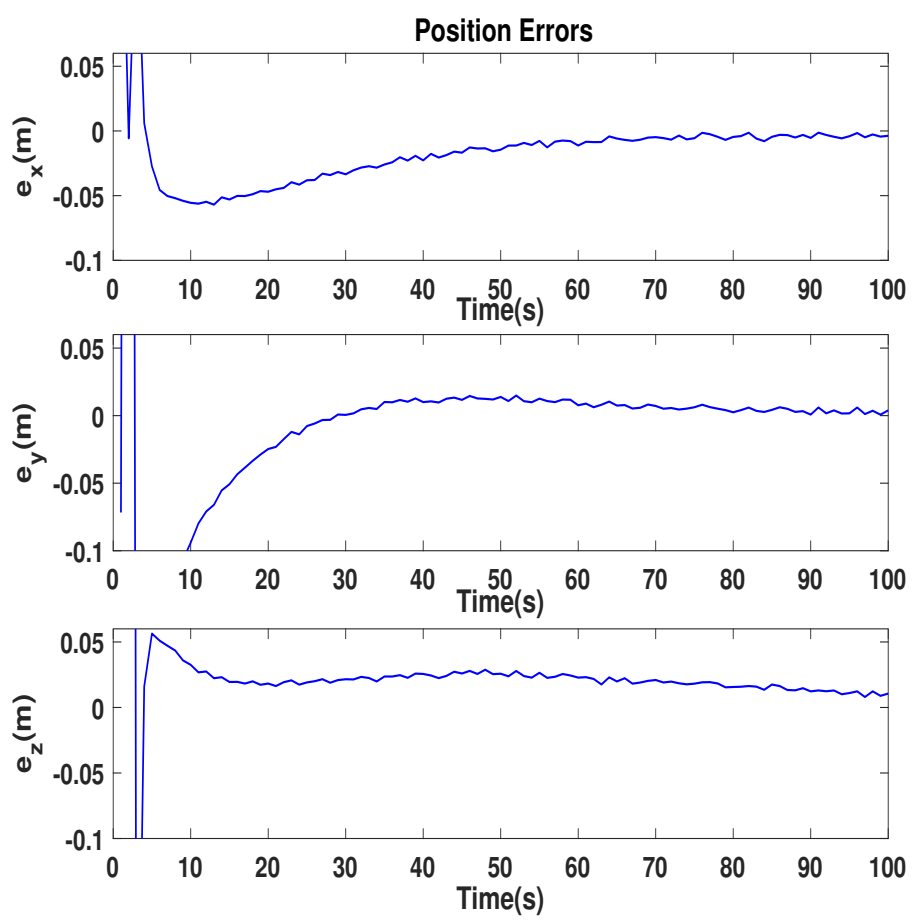

Figure. 4.4. Relative position errors [6].

\subsubsection{Results}

The relative linear position errors are shown in the Figure. 4.4. As indicated, the estimated position errors converged to near zero. The linear and angular velocities are displayed in Figure. 4.5 and Figure 4.6, respectively. In 2 secs, the estimated relative linear velocity error is less than $0.002 \mathrm{~m} / \mathrm{s}$, and the accuracy of the estimated angular velocity errors are less than $0.004 \mathrm{rad} / \mathrm{s}$ after $40 \mathrm{sec}$. The quaternion errors are plotted in Figure. 4.7. The estimated attitude reaches errors of 0.009 rad after 3 sec. At the end of each iteration, the target's quaternion was recovered from MRPs and used in Equation (4.13) for the next iteration. Hence the quaternion errors are presented to validate the performance of the suggested approach. 

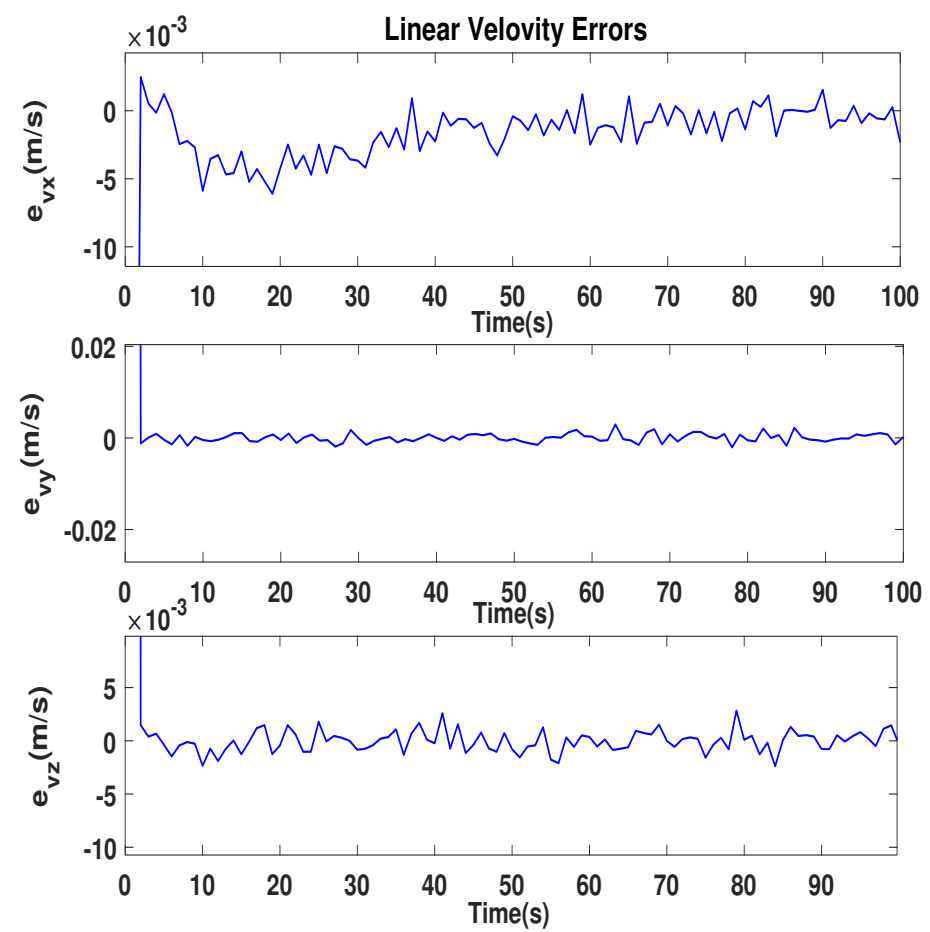

Figure. 4.5. Relative linear velocity errors [6].
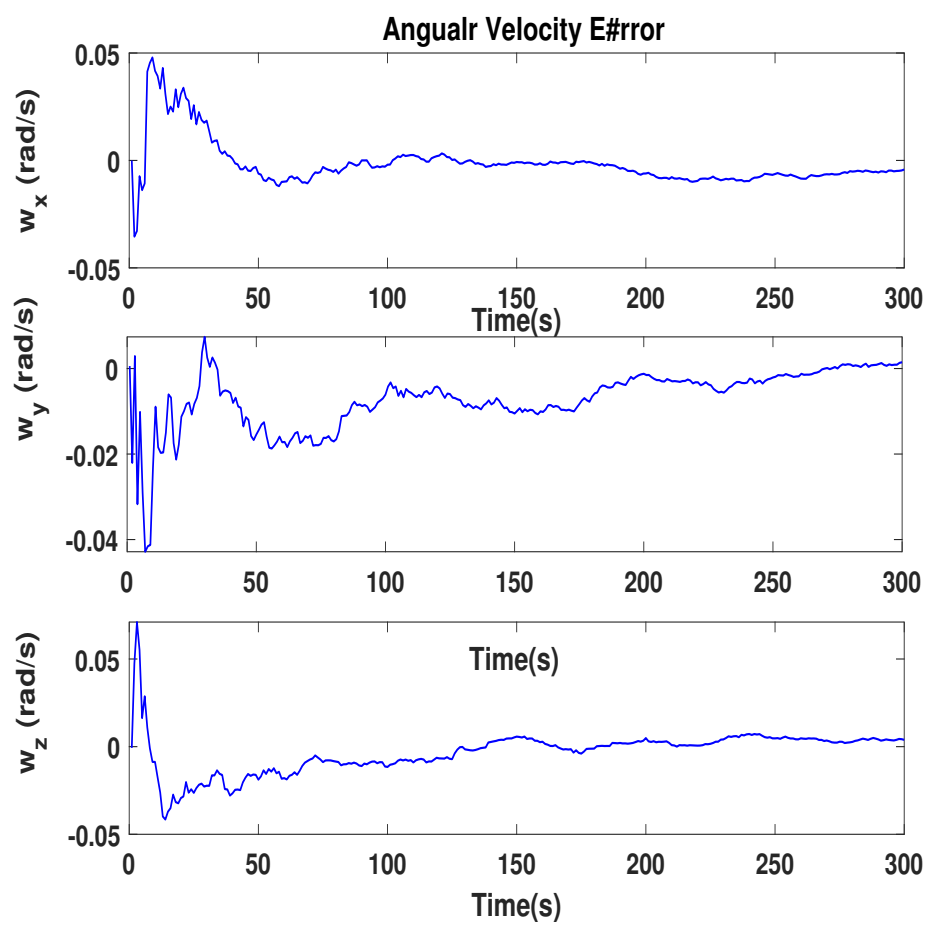

Figure. 4.6. Relative angular velocity errors [6]. 

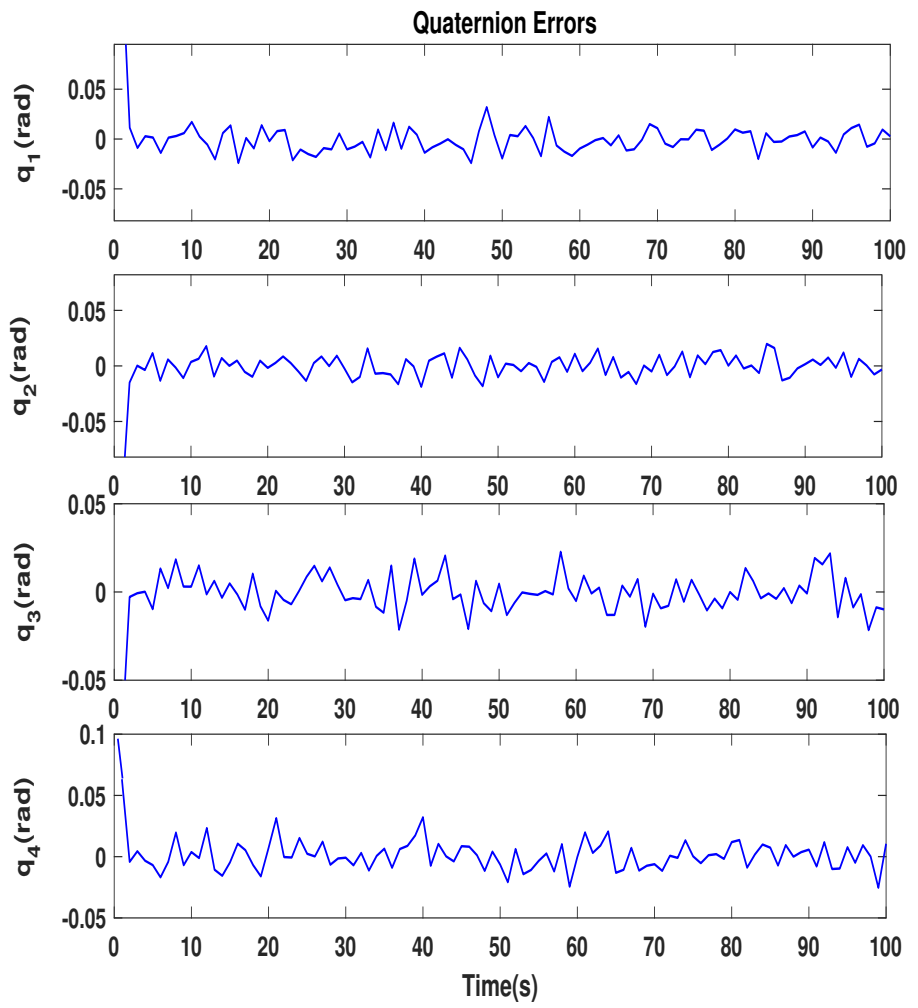

Figure. 4.7. Quaternion errors [6]. 

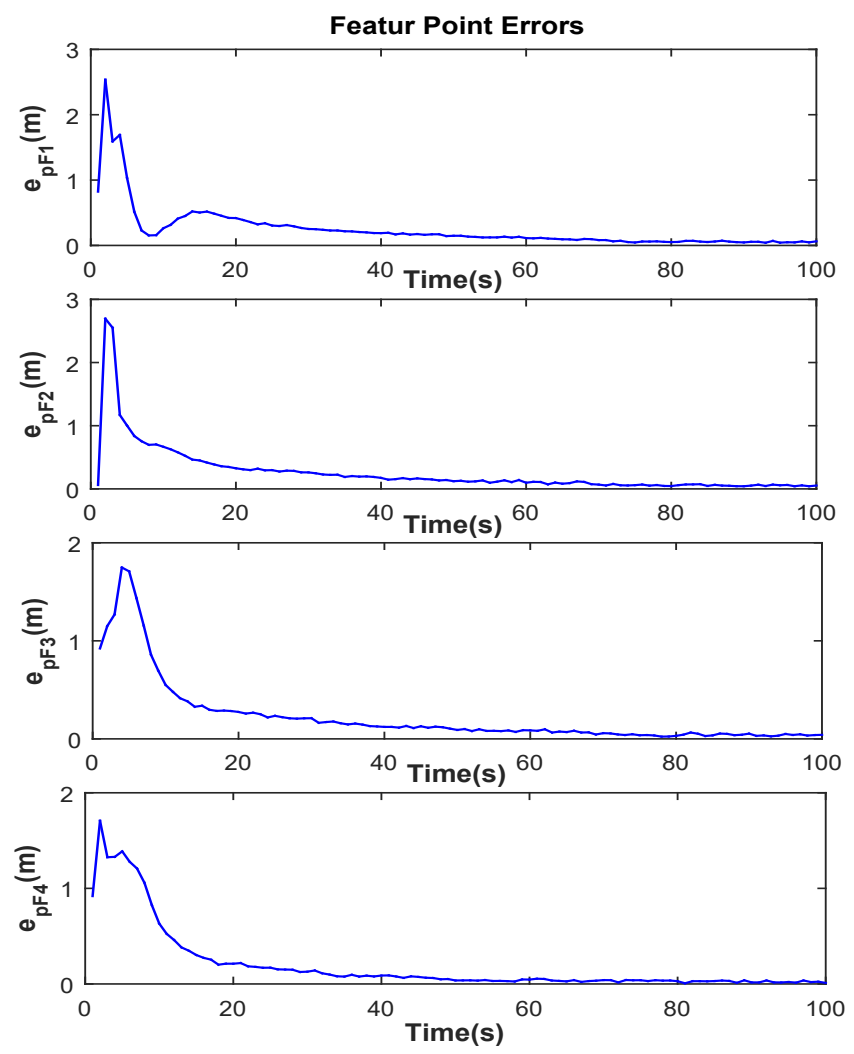

Figure. 4.8. Feature points position errors [6].

Figure. 4.8 illustrates the feature point estimation errors obtained from Equation (4.50). As seen in Figure. 4.8, the feature point positions estimated error with an accuracy of $5 \mathrm{~cm}$. 


\section{Chapter 5}

\section{Sensors Fusion}

As mentioned, target identification is the goal. To remove the space debris, adequate information of the target and chaser pose and motion is required. To obtain this required information, multiple sensors are employed. In harsh environments, feedback from one sensor is typically not adequate, specifically for implementing control algorithms. Sensor fusion can also help compensate for deficiencies in information, sensor noise, limited accuracy, failure, or a lack of knowledge about a particular aspect of the environment, chaser, and target. Due to the high uncertainty and noise level in space, the multi-sensor integration is especially challenging with the current state-of-art algorithms, and therefore advanced methods are required. Such an advanced algorithm should be able to fulfill the requirements of space environments and work with high initial uncertainty, different noise levels and outliers. This chapter describes the development of two novel integration algorithms. The first approach is based on a combination of Fuzzy Logic Controllers (FLCs) and conventional KFs, such as IEKF and UKF, to provide reliable and accurate navigation solutions. The method consists of two Fuzzy logic adaptation mechanisms incorporated with the UKF and IEKF and designed to detect KFs divergence and implement an appropriate tuning parameter by monitoring the residual or innovation, thereby preventing 
the filter from becoming unstable. The proposed algorithm is based on the correction of both the process noise covariance matrix, $\boldsymbol{Q}_{k}$, and the measurement error covariance, $\boldsymbol{R}_{k}$. The second approach is based on the $\mathrm{H}_{\infty}$ Extended Kalman filter. Despite the advantages of the $\mathrm{H}_{\infty}$ filters, such as robustness and independence of noise knowledge, for optimal results more parameters need to be tuned. Besides, when disturbances or uncertainties intensify, adjusting the threshold value can be challenging. To address these problems, the novel adaptive $\mathrm{H}_{\infty} \mathrm{EKF}$ is proposed in this work. To protect the filter from divergence when there is high initial uncertainty in the covariance matrices, adaptive factors have been designed to tune $\boldsymbol{P}_{k}, \boldsymbol{R}_{k}$ and $\boldsymbol{Q}_{k}$ matrices. Both adaptive approaches are applied to fuse data from GPS and INS. Their performance in terms of robustness against high initial uncertainty, non-zero mean noises and outlier, compared with EKF, UKF and $\mathrm{H}_{\infty} \mathrm{EKF}$.

\subsection{Introduction}

Data fusion techniques have been widely used in multi-sensory environments to combine data from multiple sensors in order to achieve fewer detection errors and higher reliability. One of the most popular approaches for navigation sensor fusion is to apply Kalman filters. A significant difficulty when designing standard Kalman Filters such as EKF and UKF and IEKF, is the divergence problem of KFs due to minimal knowledge of the models or noises [210-212]. Incomplete prior information of covariance matrices and limited knowledge of the models can seriously reduce KFs' performance and make the filters unstable [213]. Unlike KFs which require detailed knowledge of the noise, $\mathrm{H}_{\infty}$ filters require no prior assumption of process or measurement noises, and are more robust than standard KFs [214]. Many types of $\mathrm{H}_{\infty}$ filter solutions have been used for different tasks, such as game theory [215], frequency domain [216] 
and Krein-space approach in terms of the state space form [217]. The Krein space approach is used for linearized systems and can also be applied for non-linear systems based on the EKF [218]. Despite the good performance of $\mathrm{H}_{\infty}$ filters there are many circumstances, including model uncertainty and environmental disturbances, that result in information fusion hindrance and low estimation accuracy [219]. Besides, inaccurate estimates of initial values can also make the filter unstable. To address these limitations, adaptive approaches have proved to be an effective strategy [220]. Various types of adaptive algorithms have been developed, such as innovation and residual-based adaptive KFs [221,222], for which only the adaption of the measurement noise and gain matrix is considered. Others [223] also use an adaptive EKF, in which adaptation of both measurement and process covariance matrices are considered. Though multiple-model-based adaptive estimation (MMAE) can somehow overcome the model uncertainty by implementing multiple banks of KFs, the computational burden would impact system performance [224]. An adaptive EKF for the localization of mobile robots was developed by Jetto and Longhi [225]. In their work, the data provided by sonar and odometrical sensors were fused through an adaptive EKF to provide online estimates of a robot's position. An adaptive twostage EKF for estimating unknown fault bias in an INS/GPS loosely coupled system was proposed by Kim and Lee [226]. An adaptive EKF using artificial neural networks was developed by Stubberuet et al. [227], who designed a neuro-observer that can learn system uncertainties and improve the overall performance of an uncertain control system in the state-estimator model. An adaptive UKF algorithm for target tracking with unknown process noise statistics was introduced by Shi et al. [228]. A modified Sage-Husa noise statistics estimator was introduced to assess the system process noise variance adaptively in this algorithm. An adaptive fading UKF with Q-Adaptation for attitude estimation was introduced by Soken et al. [229]. A Fuzzy 
adaptive $\mathrm{H}_{\infty}$ filter, an adaptive Fuzzy hybrid UKF and a robust $\mathrm{H}_{\infty}$ filter were considered by [218,230-232], respectively. In these works, the threshold values are tuned adaptively. However, the adaption of $\boldsymbol{R}_{k}$ and $\boldsymbol{Q}_{k}$ are not considered, which can affect system accuracy when high initial uncertainties are intensified.

\section{$5.2 \quad$ System Description}

The non-linear dynamic and measurement models are given by:

$$
\begin{gathered}
\boldsymbol{x}_{k}=f\left(\boldsymbol{x}_{k-1}\right)+\boldsymbol{G}_{k} \boldsymbol{w}_{k} \\
\boldsymbol{y}_{k}=\boldsymbol{h}\left(x_{k}\right)+\boldsymbol{v}_{k}
\end{gathered}
$$

where $\boldsymbol{x}_{k}$ and $\boldsymbol{y}_{k}$ are the state and measurement vectors, respectively. $\boldsymbol{v}_{k}$ is the measurement noise and $w_{k}$ represents the process noise of the $\boldsymbol{R}_{k}$ and $\boldsymbol{Q}_{k}$ covariance matrices, respectively $[210,211] . \boldsymbol{f}($.$) and \boldsymbol{h}($.$) are non-linear functions that are used$ to estimate the predicted state and measurement.

\subsection{Fuzzy Adaptive Filters}

Conventional Kalman filters provide an effective way to estimate the state of a system from noisy measurements when the covariances of the system are known, and the system is well defined. However, there are uncertainties in the system noise covariances in some cases, and they can cause filters to become unstable. Here to protect conventional Kalman filters from divergence when there are uncertainties in the system noise covariances, exponential data weighting [233] is applied. Two new adaptive formulations, weighted IEKF and weighted UKF were developed. The IEKF is adopted 
due to its ease of implementation and relatively good performance [209]. And the UKF was used for estimation as the UKF tends to be more robust and more accurate than the EKF in its estimation of error in all the directions [197]. These are described in the subsections below.

\subsubsection{Weighted Iterated Extended Kalman Filter}

The model and implementation equations for the weighted IEKF are defined as the following recursive equations:

\section{Initialization}

$$
\hat{\boldsymbol{x}}_{0 \mid 0}=E\left(\boldsymbol{x}_{0}\right), \quad \boldsymbol{P}_{0}=E\left[\left(\boldsymbol{x}_{0}-\hat{\boldsymbol{x}}_{0 \mid 0}\right)\left(\boldsymbol{x}_{0}-\hat{\boldsymbol{x}}_{0 \mid 0}\right)^{T}\right]
$$

\section{Prediction}

The predicted state can be defined as:

$$
\hat{\boldsymbol{x}}_{k \mid k-1}=\boldsymbol{f}\left(\hat{\boldsymbol{x}}_{k-1 \mid k-1}\right)
$$

The covariance matrices of the adaptive IEKF defined as [233]:

$$
\begin{aligned}
& \boldsymbol{R}_{k}=\alpha^{-2(k+1)} \boldsymbol{R} \\
& \boldsymbol{Q}_{k}=\alpha^{-2(k+1)} \boldsymbol{Q}
\end{aligned}
$$

where $\alpha$ denotes the tuning parameter. For $\alpha>1$, as the time increases the covariance matrices decrease, which gives the recent data more credibility due to the exponential decreased noise covariance over time. When $\alpha=1$ the filter is acting like a regular 
IEKF. It should be noted that $\alpha$ is the output of the Fuzzy controllers. The error covariance defined as:

$$
\boldsymbol{P}_{k}^{-}=\boldsymbol{F}_{k-1} \boldsymbol{P}_{k-1}^{-} \boldsymbol{F}_{k-1}^{T}+\alpha^{-2(k+1)} \boldsymbol{Q}
$$

where, $\boldsymbol{F}_{k-1}$, the linear approximation equation can be present in form of:

$$
\left.\boldsymbol{F}_{k-1} \approx \frac{\partial \boldsymbol{f}}{\partial \boldsymbol{x}}\right|_{\hat{\boldsymbol{x}}_{k-1 \mid k-1}}
$$

\section{Update}

By defining the weighted covariance as:

$$
\boldsymbol{P}_{k}^{-}=\alpha^{-2 k} \boldsymbol{P}_{k \mid k-1}
$$

The Kalman gain can be computed as:

$$
\boldsymbol{K}_{k, i}=\boldsymbol{P}_{k}^{-} \boldsymbol{H}_{k, i}^{T}\left(\boldsymbol{H}_{k, i} \boldsymbol{P}_{k}^{-} \boldsymbol{H}_{k, i}^{T}+\alpha^{-2(k+1)} \boldsymbol{R}\right)^{-1}
$$

The superscript $i,(i=0,1, \ldots, \tau)$ is the number of iteration steps. By using Equation (5.9) the Kalman gain can be rewritten as:

$$
\boldsymbol{K}_{k, i}=\boldsymbol{P}_{k \mid k-1} \boldsymbol{H}_{k, i}^{T}\left(\boldsymbol{H}_{k, i} \boldsymbol{P}_{k \mid k-1} \boldsymbol{H}_{k, i}^{T}+\boldsymbol{R} / \alpha^{2}\right)^{-1}
$$

where

$$
\left.\boldsymbol{H}_{k, i} \approx \frac{\partial \boldsymbol{h}}{\partial \boldsymbol{x}}\right|_{\hat{\boldsymbol{x}}_{k-1 \mid k-1, i}}
$$

The predicted measurement estimation can be rewritten as: 


$$
\hat{\boldsymbol{x}}_{k \mid k, i+1}=\hat{\boldsymbol{x}}_{k \mid k-1}+\boldsymbol{K}_{k, i}\left(\boldsymbol{y}_{k}-\boldsymbol{h}\left(\hat{\boldsymbol{x}}_{k, i}\right)-\boldsymbol{H}_{k, i}\left(\hat{\boldsymbol{x}}_{k \mid k-1}-\hat{\boldsymbol{x}}_{k, i}\right)\right)
$$

where, $\hat{\boldsymbol{x}}_{k, i}$ presents the estimate at time point $k$ and $i_{t h}$ iteration.

The posterior covariance matrix defined as:

$$
\boldsymbol{P}_{k, i}=\left(\boldsymbol{I}-\boldsymbol{K}_{k, i} \boldsymbol{H}_{k, i}\right) \boldsymbol{P}_{k \mid k-1}
$$

The iterative process will end when a certain termination condition is met.

\subsubsection{Weighted Unscented Kalman Filter}

\section{Initialization}

$$
\hat{\boldsymbol{x}}_{0 \mid 0}=E\left(\boldsymbol{x}_{0}\right), \quad \boldsymbol{P}_{0}=E\left[\left(\boldsymbol{x}_{0}-\hat{\boldsymbol{x}}_{0 \mid 0}\right)\left(\boldsymbol{x}_{0}-\hat{\boldsymbol{x}}_{0 \mid 0}\right)^{T}\right]
$$

\section{Calculate sigma points}

For the $L$ elements state vector, a set of $(2 L+1)$ sigma-points are created according to the following:

$$
\boldsymbol{\chi}_{k-1}=\left[\begin{array}{lll}
\hat{\boldsymbol{x}}_{k-1} & \hat{\boldsymbol{x}}_{k-1}+\sqrt{(L+\lambda)} \sqrt{\boldsymbol{P}_{k-1}} & \hat{\boldsymbol{x}}_{k-1}-\sqrt{(L+\lambda)} \sqrt{\boldsymbol{P}_{k-1}}
\end{array}\right]
$$

where each column of $\boldsymbol{\chi}_{k-1}$, represents a sigma-point, $\sqrt{\boldsymbol{P}_{k-1}}=\operatorname{chol}\left(\boldsymbol{P}_{k-1}\right)$ is the square root of the state error covariance, and the scaling parameter $\lambda$ defined as:

$$
\lambda=\sigma^{2}(L+\kappa)-L
$$


where $\sigma$ and $e^{-4} \leq \sigma \leq 1$ determine the size of the sigma-points distribution, and $\kappa$ influences the accuracy of the approximation. Once the sigma-points are generated, each point is propagated throughout the non-linear state equation as:

$$
\chi_{k \mid k-1}^{(i)}=\boldsymbol{f}\left(\boldsymbol{\chi}_{k-1}, \boldsymbol{u}_{k}\right), \quad i=0,1, \ldots, 2 L
$$

The mean and covariance are approximated using a weighted mean and covariance of the transformed points as:

$$
\begin{gathered}
\hat{\boldsymbol{x}}_{k \mid k-1}=\sum_{i=0}^{2 L} \boldsymbol{\eta}_{i}^{(m)} \boldsymbol{\chi}_{k \mid k-1}^{(i)} \\
\boldsymbol{P}_{k \mid k-1}=\sum_{i=0}^{2 L} \boldsymbol{\eta}_{i}^{(c)}\left(\boldsymbol{\chi}_{k \mid k-1}^{(i)}-\hat{\boldsymbol{x}}_{k \mid k-1}\right)\left(\boldsymbol{\chi}_{k \mid k-1}^{(i)}-\hat{\boldsymbol{x}}_{k \mid k-1}\right)^{T}+\alpha^{-2(k+1)} \boldsymbol{Q}
\end{gathered}
$$

where the mean weight vector $\boldsymbol{\eta}_{i}^{(m)}$ and the covariance weight vector $\boldsymbol{\eta}_{i}^{(c)}$ associated with the $i_{t h}$ point are defined as:

$$
\begin{gathered}
\boldsymbol{\eta}_{i}^{(c)}=\boldsymbol{\eta}_{i}^{(m)}=1 /(2(L+\lambda)), \quad i=1,2, \ldots, 2 L \\
\boldsymbol{\gamma}_{k \mid k-1}^{(i)}=\boldsymbol{h}\left(\boldsymbol{\chi}_{k \mid k-1}^{(i)}\right)
\end{gathered}
$$

Then the mean of the measurement vector is calculated as:

$$
\hat{\boldsymbol{y}}_{k \mid k-1}=\sum_{i=0}^{2 L} \boldsymbol{\eta}_{i}^{(m)} \boldsymbol{\gamma}_{k \mid k-1}^{(i)}
$$

\section{Update}

Covariance and cross-covariance matrices of the proposed adaptive UKF are defined as: 


$$
\begin{aligned}
& \boldsymbol{P}_{k}^{y y}=\sum_{i=0}^{2 L} \eta_{i}^{(c)}\left(\boldsymbol{\gamma}_{k \mid k-1}^{(i)}-\hat{\boldsymbol{y}}_{k \mid k-1}\right)\left(\boldsymbol{\gamma}_{k \mid k-1}^{(i)}-\hat{\boldsymbol{y}}_{k \mid k-1}\right)^{T}+\alpha^{-2} \boldsymbol{R} \\
& \boldsymbol{P}_{k}^{x y}=\alpha^{-2(k-1)} \sum_{i=0}^{2 L} \eta_{i}^{(c)}\left(\boldsymbol{\chi}_{k \mid k-1}^{(i)}-\hat{\boldsymbol{x}}_{k \mid k-1}\right)\left(\boldsymbol{\gamma}_{k \mid k-1}^{(i)}-\hat{\boldsymbol{y}}_{k \mid k-1}\right)^{T}
\end{aligned}
$$

Similar to the weighted IEKF, when $\alpha=1$ the filter is functioning as a regular UKF. For $\alpha>1$, as the time increases, the covariance matrices decrease.

$$
\boldsymbol{K}_{k}=\boldsymbol{P}_{k}^{x y}\left(\boldsymbol{P}_{k}^{y y}\right)^{-1}
$$

The Kalman gain $\boldsymbol{K}_{k}$, is then used to update the state and covariance estimates as:

$$
\begin{gathered}
\hat{\boldsymbol{x}}_{k}=\hat{\boldsymbol{x}}_{k \mid k-1}+\boldsymbol{K}_{k}\left(\boldsymbol{y}_{k}-\hat{\boldsymbol{y}}_{k \mid k-1}\right) \\
\boldsymbol{P}_{k}=\boldsymbol{P}_{k \mid k-1}-\boldsymbol{K}_{k} \boldsymbol{P}_{k}^{y y} \boldsymbol{K}_{k}^{T}
\end{gathered}
$$

\subsection{Fuzzy Logic Controllers}

Adaptive tuning of conventional Kalman filters via the Fuzzy logic is a promising strategy to protect the filters from divergence when dealing with parameter uncertainty and non-white process noise. The FLCs continuously monitor and tune the noise level in the filters' internal models to adjust the filters. The innovation sequence of Kalman filtering should be zero-mean white noise processes; if not divergence will occur. Hence, in this thesis the covariance and mean values of the innovation sequence are used as inputs to the FLCs to determine the degrees of divergence. The proposed Fuzzy adaptive Kalman filters have been developed and validated for two different cases. 


\subsubsection{Fuzzy Logic Adaptive Mechanism for Parameter Un- certainty}

The uncertain or time-varying parameters in the $\boldsymbol{Q}$ and $\boldsymbol{R}$ matrices make the conventional Kalman filters diverge, or cover an overly large range. When the filter does not work well, the FLCs apply a suitable weighting factor to enhance the accuracy. Here two groups of Fuzzy controllers have been defined for parameter uncertainty.

\section{First Fuzzy Controller}

In this controller, the mean values and covariance of the innovation are inputs and used to determine the degree of divergence. The exponential weighted $\alpha$ is the first Fuzzy controller output. By selecting a suitable $\alpha$ the FLC adapt UKF and IEKF optimally.

The membership function of FLC inputs (mean and covariance of the innovation sequence) and the output $\alpha$ are illustrated in Figures. 5.1, 5.2 and 5.3, respectively. The characteristics of a Fuzzy system are highly dependent on the relevant rules; this work proposed a Fuzzy logic controller with nine rules, as shown in Table 5.1. Generally, when the covariance is becoming large, and mean value is moving away from zero, the Kalman filters are becoming unstable. In this case, a large $\alpha$ will be applied. A large $\alpha$ means that process noises are added. It can ensure that in the model all states are sufficiently excited by the process noise. When the covariance is extremely large, there are some problems with the GPS measurements, so the filter cannot depend on these measurements anymore, and a smaller $\alpha$ will be used. By selecting a proper, $\alpha$, the fuzzy logic controller will adapt the Kalman filter optimally 


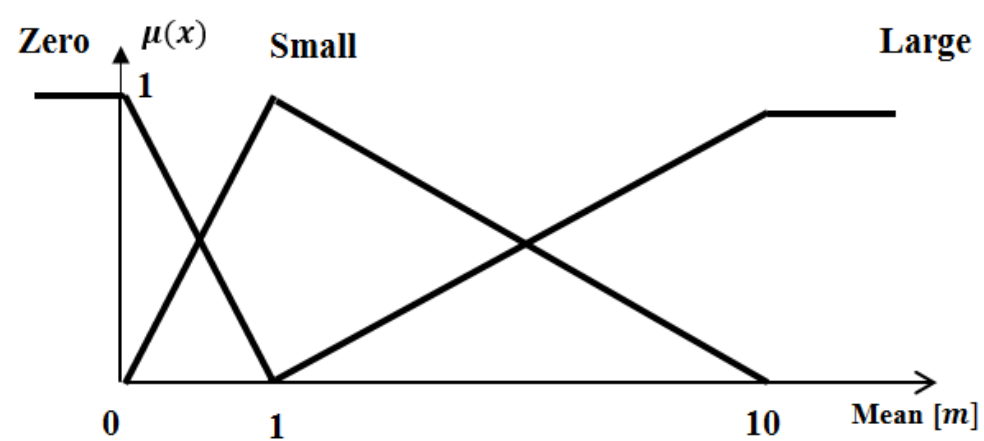

Figure. 5.1. Mean value membership functions for parameter uncertainty [1,2].

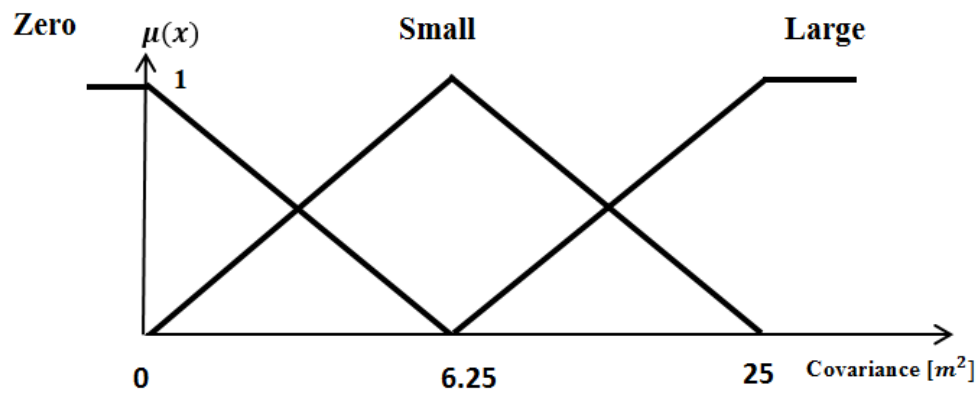

Figure. 5.2. Covariance membership functions for parameter uncertaint $[1,2]$.

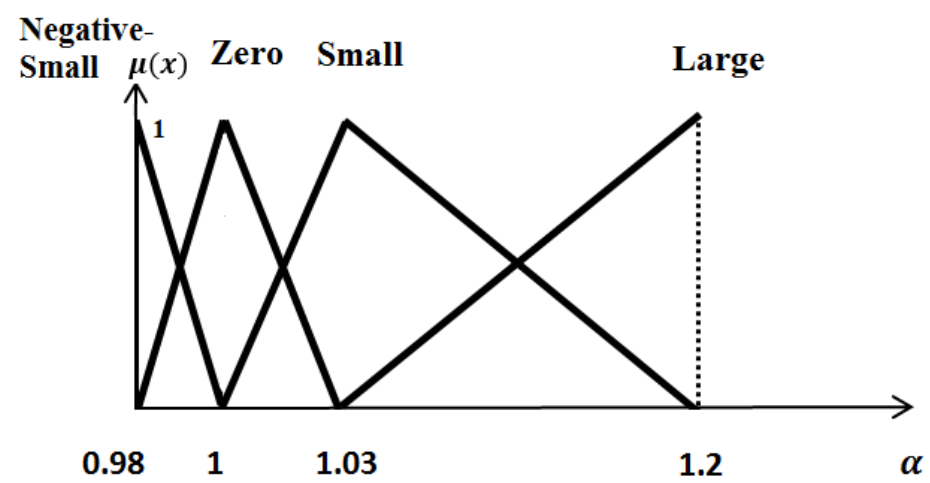

Figure. 5.3. $\alpha$ membership functions for parameter uncertainty $[1,2]$.

and try to keep the innovation sequence acting as zero-mean white noise.

\section{Second Fuzzy Controller}

The second controller was designed to detect changes in the $\boldsymbol{R}$ matrix to adjust the filter accordingly. The measurement covariance matrix $\boldsymbol{R}$ is related to innovation 
Table 5.1: Rule table of $\alpha$ for parameter uncertainty [1,2].

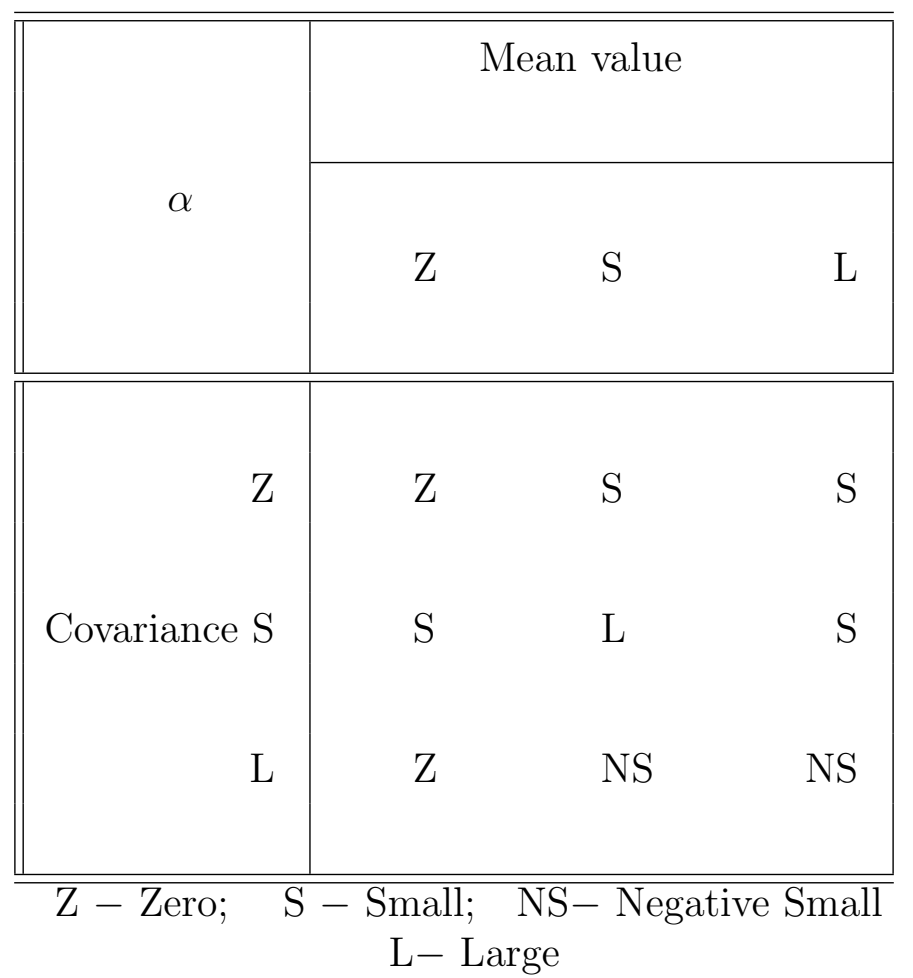

sequence covariance; thus, any changes in the measurement covariance matrix can alter the covariance of the innovation sequence. With this controller, the UKF and IEKF are adapted by selecting the appropriate scale. For example, the FLC applies a large scale to adjust the $\alpha$ if it determines that the innovation's covariance is greater than expected.

$$
\alpha^{\text {adjust }}=(\alpha-1) \times \text { scale }+1
$$

The nine rules for this Fuzzy controller are shown in Table 5.2. 
Table 5.2: Rule table of scale for for parameter uncertainty [1,2].

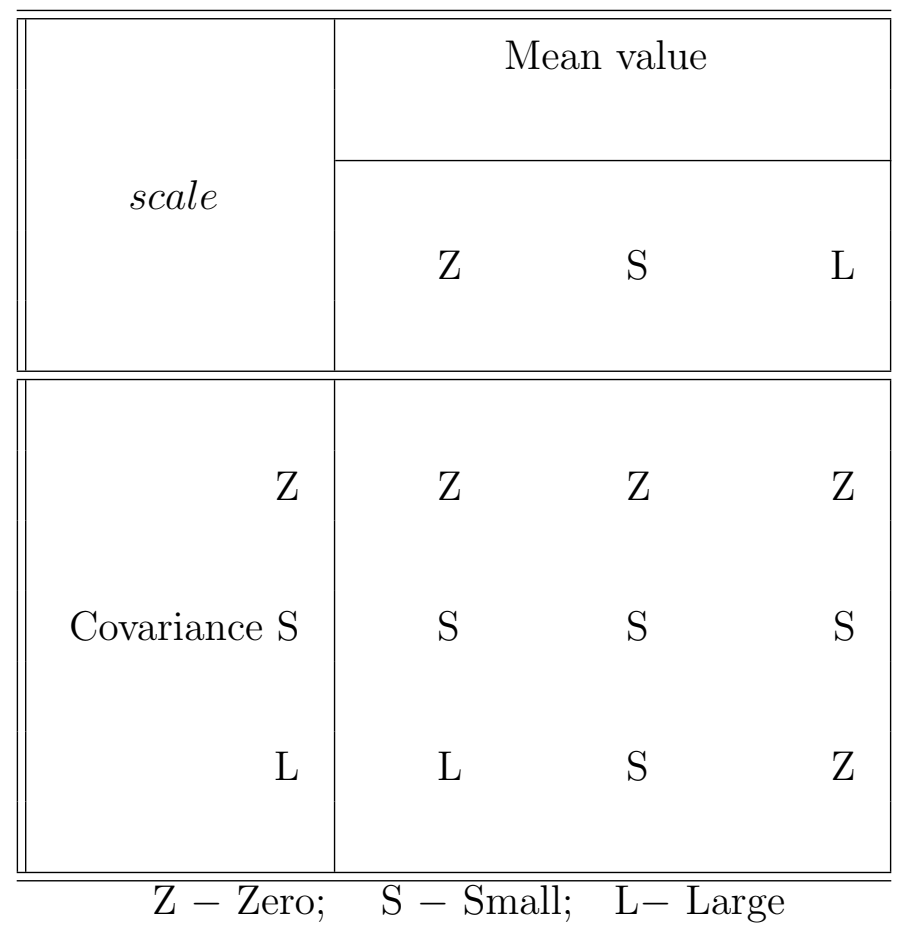

\subsubsection{Fuzzy Logic Adaptive Mechanism for Non-white Pro- cess Noise}

The conventional Kalman filters require that the process noise $\boldsymbol{w}_{k}$ be zero-mean white noise with known covariances of $\boldsymbol{Q}$. In practice, the process noise could be correlated with itself, and applying the FLC is an alternative to this problem. To adapt the Kalman filter a Fuzzy controller was design to apply a proper tuning parameter and prevent the filter from divergence. The membership functions for this fuzzy controller are shown in Figures. 5.4, 5.5 and 5.6, respectively. There are nine rules for the FLC when it encounters non-white noise (see Table 5.3); thus, minimal computational time is required. 


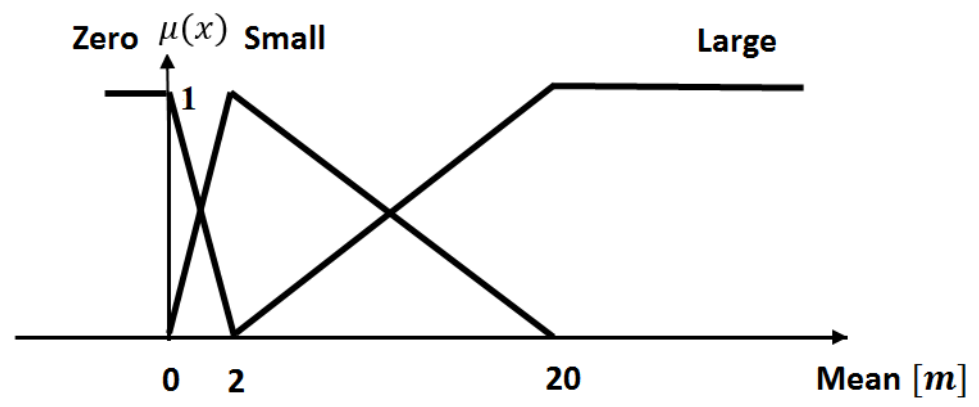

Figure. 5.4. Mean value membership functions for non-zero mean process noise $[1,2]$.

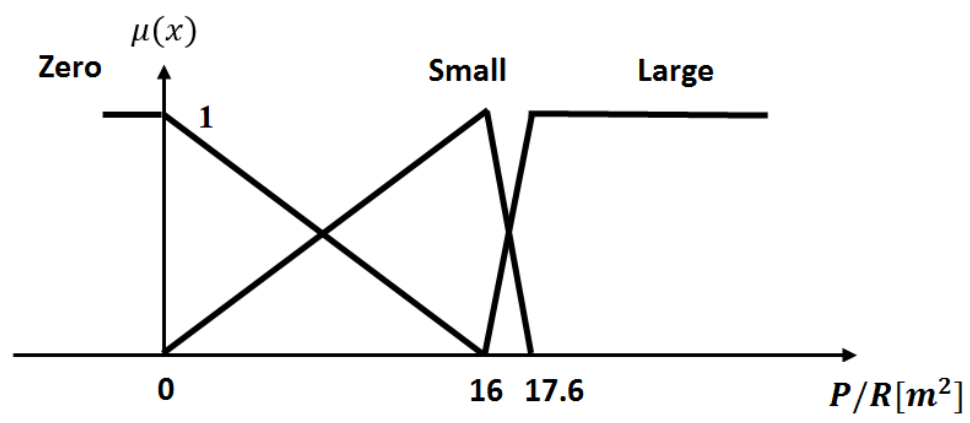

Figure. 5.5. Covariance membership functions for non-zero mean process noise $[1,2]$.

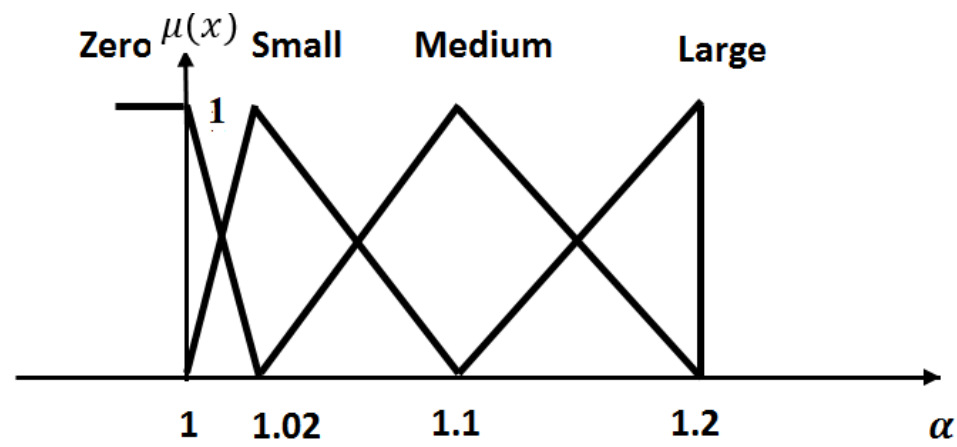

Figure. 5.6. $\alpha$ membership functions for non-zero mean process noise $[1,2]$. 
Table 5.3: Rule table of $\alpha$ for non-white noise [1,2].

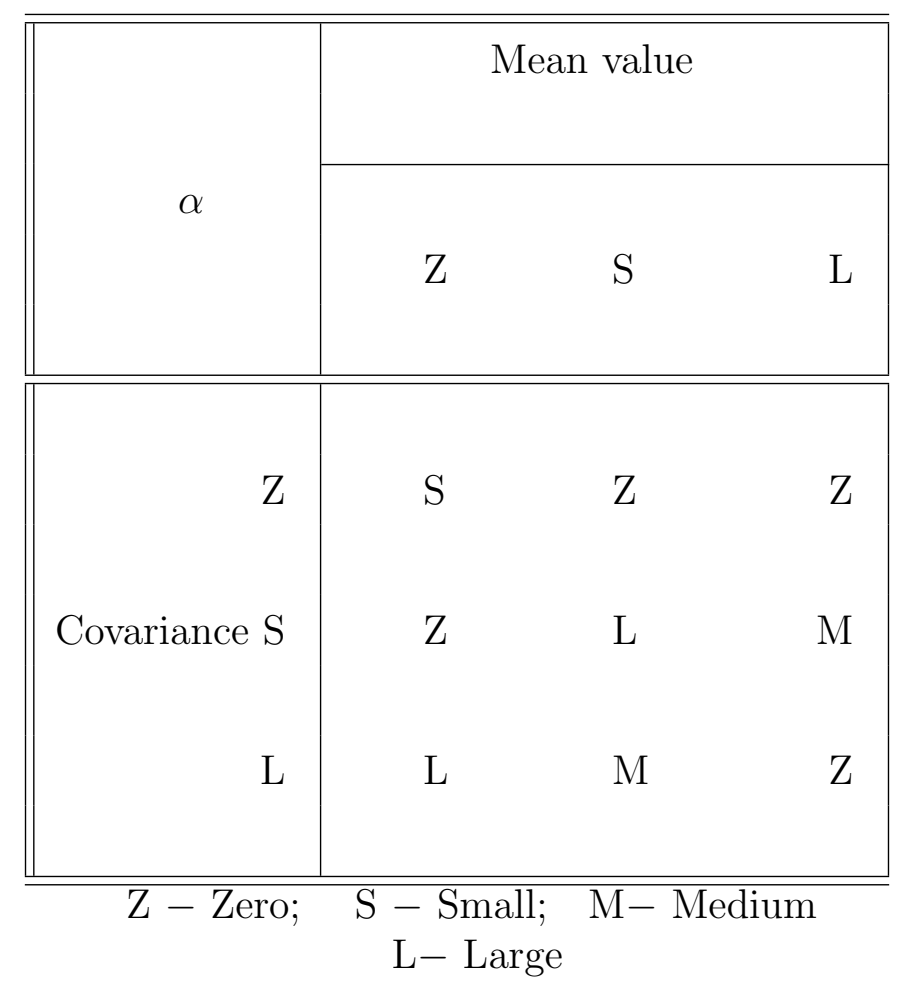

The Innovation sequence of the proposed filters, FAUKF and FAIEKF when there is nonzero mean $\mu=1$, in meter are shown in Figure. 5.7. The innovation is the difference between the best measurement prediction based on the filter's internal mode and the actual measurement; thus, it can be used to evaluate the filter's performance. From Figure. 5.7 it can be seen that the innovation of the IEKF has a large drift, while the innovation mean value of FAUKF is smaller and remains close to zero. As it shows the FAUKF performs better than the FAIEKF, Since the FAUKF was shown more accurate results than FAIEKF the results corresponding to the FAUKF are considered in rest of this thesis. Further information can be found in [2]. It should be noted that in order to validate the accuracy and convergence of the proposed approaches, results obtained by FAUKF and FAIEKF were also compared with the Fuzzy Adaptive Extended Kalman Filter (FAEKF) [234]. The simulation results 

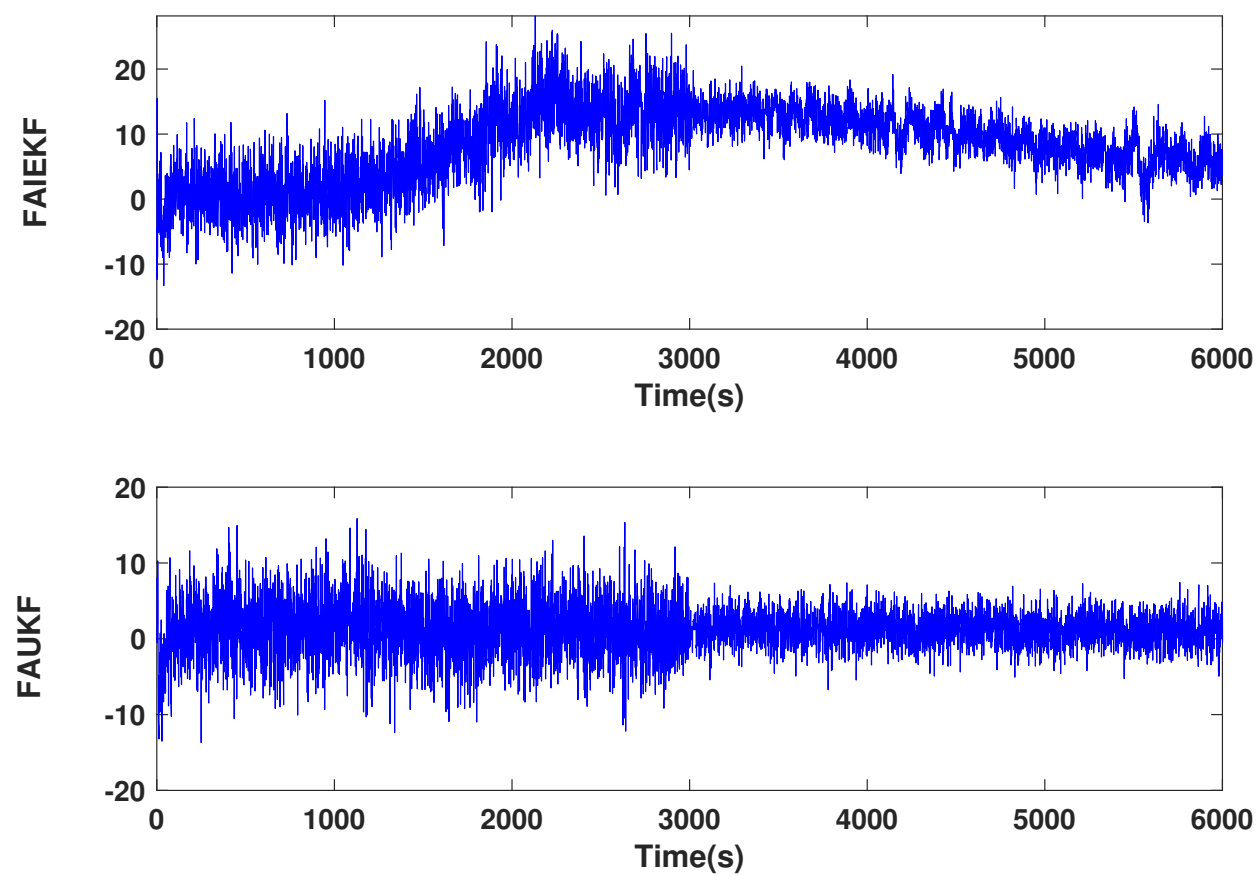

Figure. 5.7. Innovation sequences.

illustrate the superior performance of the AKUKF and FAIEKF compared to FAEKF. Further information can be found in [2].

\subsection{Robust Extended Kalman Filter}

The $\mathrm{H}_{\infty}$ Extended Kalman filter (HEKF) makes robust estimations against environmental noises, and it is widely used in industrial applications $[215,216]$. Unlike standard Kalman filters, HEKF requires no primary assumption of noise characteristics. KFs minimize the mean squared error of the estimation error while HEKF minimizes the highest estimation error, which makes it more robust than other Kalman filters such as EKF and UKF [235]. The principle of HEKF is based on its optimal estimate that guarantees the smallest energy error for all possible disturbances of the fixed 
energy.

A discrete state-space model of the standard HEKF can be written as:

$$
\begin{gathered}
\boldsymbol{x}_{k}=\boldsymbol{F}_{k} \boldsymbol{x}_{k-1}+\boldsymbol{G}_{k} \boldsymbol{w}_{k} \\
\boldsymbol{y}_{k}=\boldsymbol{H}_{k} \boldsymbol{x}_{k}+\boldsymbol{v}_{k} \\
\boldsymbol{z}_{k}=\boldsymbol{L}_{k} \boldsymbol{x}_{k}
\end{gathered}
$$

where $\boldsymbol{z}_{k}$ is a linear combination of states and $\boldsymbol{L}_{k}$ is a user-defined matrix (assumed to be full rank). If we want to directly estimate $\boldsymbol{x}_{k}, \boldsymbol{L}_{k}$ can be defined as an identity matrix $\boldsymbol{L}_{k}=\boldsymbol{I}$. In the game theory approach to $\mathrm{H}_{\infty}$ filtering the cost function of a non-linear filter is defined as follows [215]:

$$
J=\frac{\sum_{k=1}^{N}\left\|\boldsymbol{x}_{k}-\hat{\boldsymbol{x}}_{k \mid k}\right\|_{\boldsymbol{P}_{k \mid k}^{-1}}^{2}}{\left\|\boldsymbol{x}_{0}-\hat{\boldsymbol{x}}_{0 \mid 0}\right\|_{\boldsymbol{P}_{0 \mid 0}^{-1}}^{2}+\sum_{k=1}^{N}\left(\left\|\boldsymbol{\omega}_{k}\right\|_{\boldsymbol{Q}_{k}^{-1}}^{2}+\left\|\boldsymbol{v}_{k}\right\|_{\boldsymbol{R}_{k}^{-1}}^{2}\right)} \leq \gamma^{2}
$$

where $N$ is the number of filtering epochs, $\boldsymbol{x}_{0}$ is the initial value of the state vector $\boldsymbol{x}$ with the covariance matrix $\boldsymbol{P}_{k}$ and initial covariance $\boldsymbol{P}_{0 \mid 0}, \hat{\boldsymbol{x}}_{0 \mid 0}$ and $\hat{\boldsymbol{x}}_{k}$ are the estimated state vectors of $\boldsymbol{x}_{0}$ and $\boldsymbol{x}_{k}$, respectively. The goal is to find $\hat{\boldsymbol{x}}_{k \mid k}$, which minimizes the cost function $J$. However, to have an optimal HEKF estimate it is analytically critical to determine the explicit minimization of $J$. Therefore, various sub-optimal methods, such as the Krein space approach [217], are applied to address the $\mathrm{H}_{\infty}$ problems by setting the threshold as a performance bond to the cost function, as follows [218]:

$$
\sup _{\boldsymbol{x}_{0}, \boldsymbol{v}_{k}, \boldsymbol{w}_{k}} \frac{\sum_{k=1}^{N}\left\|\boldsymbol{x}_{k}-\hat{\boldsymbol{x}}_{k \mid k}\right\|_{\boldsymbol{P}_{k \mid k}^{-1}}^{2}}{\left\|\boldsymbol{x}_{0}-\hat{\boldsymbol{x}}_{0 \mid 0}\right\|_{\boldsymbol{P}_{0 \mid 0}^{-1}}^{2}+\sum_{k=1}^{N}\left(\left\|\boldsymbol{\omega}_{k}\right\|_{\boldsymbol{Q}_{k}^{-1}}^{2}+\left\|\boldsymbol{v}_{k}\right\|_{\boldsymbol{R}_{k}^{-1}}^{2}\right)} \leq \gamma^{2}
$$

where $\gamma>0$ and $\gamma^{2}$ denotes the threshold value that limits the upper threshold of 
the energy of the cost function. The smaller $\gamma^{2}$ we select, the more robust estimates we get; though it cannot be less than the optimal solution [236]. Therefore, selecting this factor plays a crucial role in designing a robust and accurate filter.

Lemma 1 [217]: HEKF cannot have a solution unless $\gamma>0$ and $\left[\begin{array}{ll}\boldsymbol{F}_{k} & \boldsymbol{G}_{k}\end{array}\right]$ is a full ranked matrix and the following inequality is satisfied for all steps $(k)$.

$$
\boldsymbol{P}_{k \mid k}^{-1}+\boldsymbol{H}_{k}^{T} \boldsymbol{R}_{k}^{-1} \boldsymbol{H}_{k}-\gamma^{-2} \boldsymbol{L}_{k}^{T} \boldsymbol{L}_{k}>0
$$

where $\boldsymbol{R}_{k}^{-1}$ is the inverse of the measurement covariance matrix, and the Riccati recursion relation is obtained from $\boldsymbol{P}_{k \mid k}$ as follows:

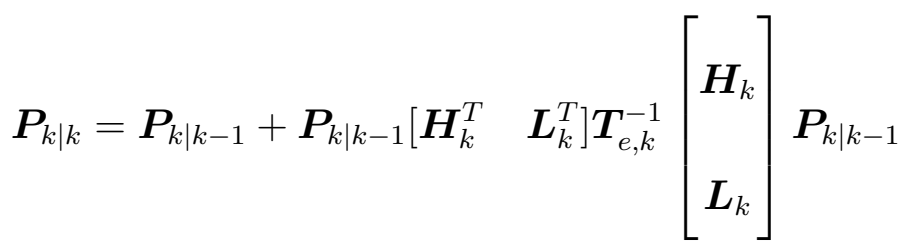

where $\boldsymbol{P}_{k \mid k-1}$ and the Krein space Gramian $\boldsymbol{T}_{e, k}$ are defined as [217]:

$$
\begin{gathered}
\boldsymbol{P}_{k \mid k-1}=\boldsymbol{F}_{k} \boldsymbol{P}_{k-1 \mid k-1} \boldsymbol{F}_{k}^{T}+\boldsymbol{Q}_{k} \\
\boldsymbol{T}_{e, k}=\left[\begin{array}{cc}
\boldsymbol{R}_{k}+\boldsymbol{H}_{k} \boldsymbol{P}_{k \mid k-1} \boldsymbol{H}_{k}^{T} & \boldsymbol{P}_{k \mid k-1} \boldsymbol{H}_{k}^{T} \\
\boldsymbol{P}_{k \mid k-1} \boldsymbol{H}_{k}^{T} & -\gamma^{2} \boldsymbol{I}+\boldsymbol{P}_{k \mid k-1}
\end{array}\right]
\end{gathered}
$$

The HEKF gain and estimate of the state vector can be written as:

$$
\begin{gathered}
\boldsymbol{K}_{k}=\boldsymbol{P}_{k \mid k-1} \boldsymbol{H}_{k}^{T}\left(\boldsymbol{H}_{k} \boldsymbol{P}_{k \mid k-1} \boldsymbol{H}_{k}^{T}+\boldsymbol{R}_{k}\right)^{-1} \\
\hat{\boldsymbol{x}}_{k \mid k}=\hat{\boldsymbol{x}}_{k \mid k-1}+\boldsymbol{K}_{k}\left(\boldsymbol{y}_{k}-\boldsymbol{H}_{k} \hat{\boldsymbol{x}}_{k \mid k-1}\right)
\end{gathered}
$$

Here, a Krein space model is used as a sub-optimal solution to address the optimal 
$\mathrm{H}_{\infty}$ problem, as its analytical solution is a complicated method to achieve [236]. The threshold value must be tuned precisely, as inaccurate tuning can cause filter divergence. In addition, incomplete a prior information can make the filter unstable. Thus, in this thesis an adaptive algorithm is designed to manage these issues and make estimates more accurate and flexible to initial uncertainties, disturbances and outliers.

\subsubsection{Adaptation of Error Covariance}

The AHEKF is developed by using an adaptive scale factor $\sigma_{k}$ in error covariance matrix $\boldsymbol{P}_{k \mid k}$ to make a trade-off between robustness and accuracy of the filter. To evaluate system errors, the measurement innovation vector $\boldsymbol{V}_{k}$ is defined as:

$$
\boldsymbol{V}_{k}=\boldsymbol{y}_{k}-\boldsymbol{H}_{k} \hat{\boldsymbol{x}}_{k \mid k-1}
$$

The covariance matrix of the innovation vector is defined as :

$$
\boldsymbol{S}_{k}=\boldsymbol{H}_{k} \boldsymbol{P}_{k-1 \mid k-1} \boldsymbol{H}_{k}^{T}+\boldsymbol{R}_{k}
$$

Two segment method [237] is used to find the optimal adaptive factor $\sigma_{k}$, such that the theoretical value of a predicted measurement is nearly equal to the actual estimated output of the filter.

$$
\sigma_{k}= \begin{cases}1 & \text { if }\left|\Delta \boldsymbol{V}_{x}\right| \leq c_{1} \\ \frac{c_{1}}{\left|\Delta \boldsymbol{V}_{x}\right|} & \text { if }\left|\Delta \boldsymbol{V}_{k}\right|>c_{1}\end{cases}
$$

where $c_{1}$ is a constant value between $[1,2.5]$ and here the best result obtained for 
$c_{1}=1.25 .\left|\triangle \boldsymbol{V}_{k}\right|$ is defined as:

$$
\left|\Delta \boldsymbol{V}_{k}\right|=\sqrt{\frac{\boldsymbol{V}_{k}^{T} \boldsymbol{V}_{k}}{\operatorname{trace}\left(\boldsymbol{S}_{k}\right)}}
$$

Consequently, the recursive form of AHEKF can be written as follows:

$$
\begin{gathered}
\boldsymbol{P}_{k \mid k-1}=\boldsymbol{F}_{k} \boldsymbol{P}_{k-1 \mid k-1} \boldsymbol{F}_{k}^{T}+\boldsymbol{Q}_{k} \\
\hat{\boldsymbol{x}}_{k \mid k}=\boldsymbol{F}_{k} \hat{\boldsymbol{x}}_{k \mid k-1}+\boldsymbol{K}_{k} \boldsymbol{V}_{k} \\
\hat{\boldsymbol{K}}_{k}=\boldsymbol{P}_{k \mid k-1} \boldsymbol{H}_{k}^{T}\left(\boldsymbol{H}_{k} \boldsymbol{P}_{k \mid k-1} \boldsymbol{H}_{k}^{T}+\boldsymbol{R}_{k}\right)^{-1} \\
\boldsymbol{P}_{k \mid k}=\frac{1}{\sigma_{k}} \boldsymbol{P}_{k \mid k-1}-\frac{1}{\sigma_{k}} \boldsymbol{P}_{k \mid k-1}\left[\boldsymbol{H}_{k}^{T} \quad \boldsymbol{L}_{k}^{T}\right] \boldsymbol{T}_{e, k}^{-1}\left[\begin{array}{c}
\boldsymbol{H}_{k} \\
\boldsymbol{L}_{k}
\end{array}\right] \boldsymbol{P}_{k \mid k-1}
\end{gathered}
$$

where

$$
\boldsymbol{T}_{e, k}=\left[\begin{array}{cc}
\boldsymbol{R}_{k}+\boldsymbol{H}_{k} \boldsymbol{P}_{k \mid k-1} \boldsymbol{H}_{k}^{T} & \left(\boldsymbol{P}_{k \mid k-1} \boldsymbol{H}_{k}^{T}\right)^{T} \\
\boldsymbol{P}_{k \mid k-1} \boldsymbol{H}_{k}^{T} & -\gamma^{-2} \boldsymbol{I}+\boldsymbol{P}_{k \mid k-1}
\end{array}\right]
$$

As mentioned previously, the adaptive factor $\sigma_{k}$ is designed to tune the filter covariance matrix $\boldsymbol{P}_{k \mid k}$ to overcome effects of various noise and disturbances. However, when there is high initial uncertainty the accuracy of the algorithm is degraded. To manage this problem, measurement and process covariance matrices can be tuned by adaptive factors, as discussed in the following subsection. 


\subsubsection{Adaptation of State and Measurement Covariance Ma- trices}

Though HEKF does not require knowing the nature of the measurement and process noise, for optimal performance the $\boldsymbol{R}_{k}$ and $\boldsymbol{Q}_{k}$ need be tuned as well. Tuning these parameters can be challenging as there is a trade-off between the robustness and accuracy of the filter.

At the predication step, the innovation is the difference between the actual measurement and its predicted value. On the other hand, the residual is the difference between actual measurement and its estimated value using the information available at step $k$. When both the $\boldsymbol{R}_{k}$ and $\boldsymbol{Q}_{k}$ matrices are estimated based on the innovation or residual covariance, $\boldsymbol{Q}_{k}$ must be estimated assuming full knowledge of the $\boldsymbol{R}_{k}$ and vice versa [238]. To run the $\boldsymbol{Q}_{k}$ and $\boldsymbol{R}_{k}$ at the same time when we have high uncertainties in both matrices, the $\boldsymbol{Q}_{k}$ adaptation method presented here estimates the $\boldsymbol{Q}_{k}$ matrix based on the innovation covariance, and the adaptation method for the $\boldsymbol{R}_{k}$ matrix is a residual covariance-based scaling method.

\section{Residual-based adaptation of $\mathbf{R}$}

Since $\boldsymbol{R}_{k}$ can be affected by distributed data from environmental observations to ensure a positive definite matrix, the residual based adaptive approach based on the Sage-Husa [239] is defined as follows:

$$
\boldsymbol{R}_{k}=\left(1-\mu_{k}\right) \boldsymbol{R}_{k-1}+\mu_{k}\left(\overline{\boldsymbol{V}}_{k} \overline{\boldsymbol{V}}_{k}^{T}+\boldsymbol{H}_{k} \boldsymbol{P}_{k \mid k-1} \boldsymbol{H}_{k}^{T}\right)
$$

where $\overline{\boldsymbol{V}}_{k}=y_{k}-\mathbf{H}_{k} \hat{x}_{k \mid k}$ denotes the residual and the tuning parameter, $\mu_{k}$, defined as: 


$$
\mu_{k}=\frac{1-\delta}{\left(1-\delta^{k}\right)}
$$

where $\delta$ is a forgetting factor. The value of is usually chosen between 0.95 to 0.998 [239], Here, the optimal solution is achieved by selecting $\delta=0.985$. The forgetting factor can limit filter memory length, and improve the effect of new observational data. That recent data plays a major role in estimation, and make the old data gradually forgotten. Additionally, to avoid disturbing the data, the threshold can be controlled by upper and lower bound quantities as:

$$
\boldsymbol{R}_{k-1} U_{l} \ll\left(H_{k} \boldsymbol{P}_{k \mid k-1} \boldsymbol{H}_{k}^{T}+\overline{\boldsymbol{V}}_{k} \overline{\boldsymbol{V}}_{k}^{T}\right) \ll \boldsymbol{R}_{k-1} U_{u}
$$

where $U_{l}$ and $U_{u}$ represent the lower and upper limit criteria, respectively. Here, as the measurement covariance matrix is adaptively tuned, subsequent disturbed or abnormal data can be avoided.

\section{Innovation Based adaptation of $\mathrm{Q}$}

Correspondingly, the process covariance matrix is modified if unmodeled dynamic errors rise significantly. Therefore, the adaption of $\boldsymbol{Q}_{k}$ is defined as:

$$
\boldsymbol{Q}_{k}= \begin{cases}\rho \boldsymbol{Q}_{k-1}+(1-\rho) \boldsymbol{K}_{k} \boldsymbol{S}_{k} \boldsymbol{K}_{k}^{T} & \left|\boldsymbol{V}_{k} \boldsymbol{V}_{k}^{T}\right|>c_{2} \\ \boldsymbol{Q}_{k-1} & \text { Otherwise }\end{cases}
$$

where $c_{2}$ is a constant value that can be set by trial and error. Here, the optimal solution is achieved by selecting $c_{2}=1.3$, and $\rho$ is defined as:

$$
\rho=\frac{\operatorname{trace}\left(\hat{\boldsymbol{C}}_{v}\right)}{\operatorname{trace}\left(\boldsymbol{S}_{k}\right)}
$$


where the $\boldsymbol{S}_{k}$ denotes theoretical covariance. The principle of the Adaptive Kalman Filter is to make the Kalman filter innovation sequence consistent with its theoretical

covariance. An estimate of the covariance of the innovation, $\hat{\boldsymbol{C}}_{v}$, is obtained by averaging the previous innovation sequence over a window length $\mathrm{M}$ as:

$$
\hat{\boldsymbol{C}}_{v}=\frac{1}{M} \sum_{i=1}^{M-1} \boldsymbol{V}_{k-i} \boldsymbol{V}_{k-i}^{T}
$$

This tuning parameter allows $\boldsymbol{Q}_{k}$ to be tuned adaptability. If $\hat{\boldsymbol{C}}_{v}$ and $\boldsymbol{S}_{k}$ matrices are consistent, the adaptive factor $\rho$ will be equal to one and $\boldsymbol{Q}_{k}=\boldsymbol{Q}_{k-1}$; otherwise, there is a problem and the covariance matrix, $\boldsymbol{Q}_{k}$ needs to be tuned to prevent the filter from divergence.

\subsection{Simulation Experiment}

The simulation experiment was performed to evaluate the performance of the proposed AHEKF and FAUKF. These algorithms could be utilized for the identification of the target, localization of chaser or even the vehicle (rocket, plane, etc.) carrying the chaser to its final destination. In this thesis, the proposed sensor fusion algorithms are validated for INS and GPS sensors. These two sensors were chosen as GPS/INS are using in many spacecraft proximity operations [240].

The GPS-aided inertial error model described in [241] was used as the state equations. The state vectors are made up of eight states: three for position errors, three for velocity errors, and two for GPS range bias and drift.

$$
\boldsymbol{x}_{k}=\left[x_{k}, y_{k}, z_{k}, \dot{x}_{k}, \dot{y}_{k}, \dot{z}_{k}, c \Delta t, c \dot{\Delta} t\right]
$$

where $x$ points east, $y$ points north, and $z$ in the attitude, $c \Delta t, c \dot{\Delta} t$ represent the GPS 
range and drift states, respectively. To correct the error of INS, GPS measurements are used to estimate the inertial system errors, subtract them from the INS output and then obtain the corrected INS outputs. Hence, the observation processed in the Kalman filters is the difference between the actual receiver measured pseudorange and predicted measurement based on corrected INS position and corrected GPS receiver clock. The measurement model and major error sources of GPS are provided in Appendix A. Many GPS/INS integration are feed-forward or open-loop configurations, where the estimates of INS errors are not used to correct the INS. However, the reference and actual trajectories can diverge to the point where an assumption of linearity and the random process models gradually deteriorates. To address this, feedback or closed-loop configuration were chosen Here, the estimates of the INS error are used to correct the INS, which allows the filter to be used for long periods. The block diagram for the GPS/INS navigation sensor fusion using the proposed adaptive schemes is shown in Figure. 5.8.

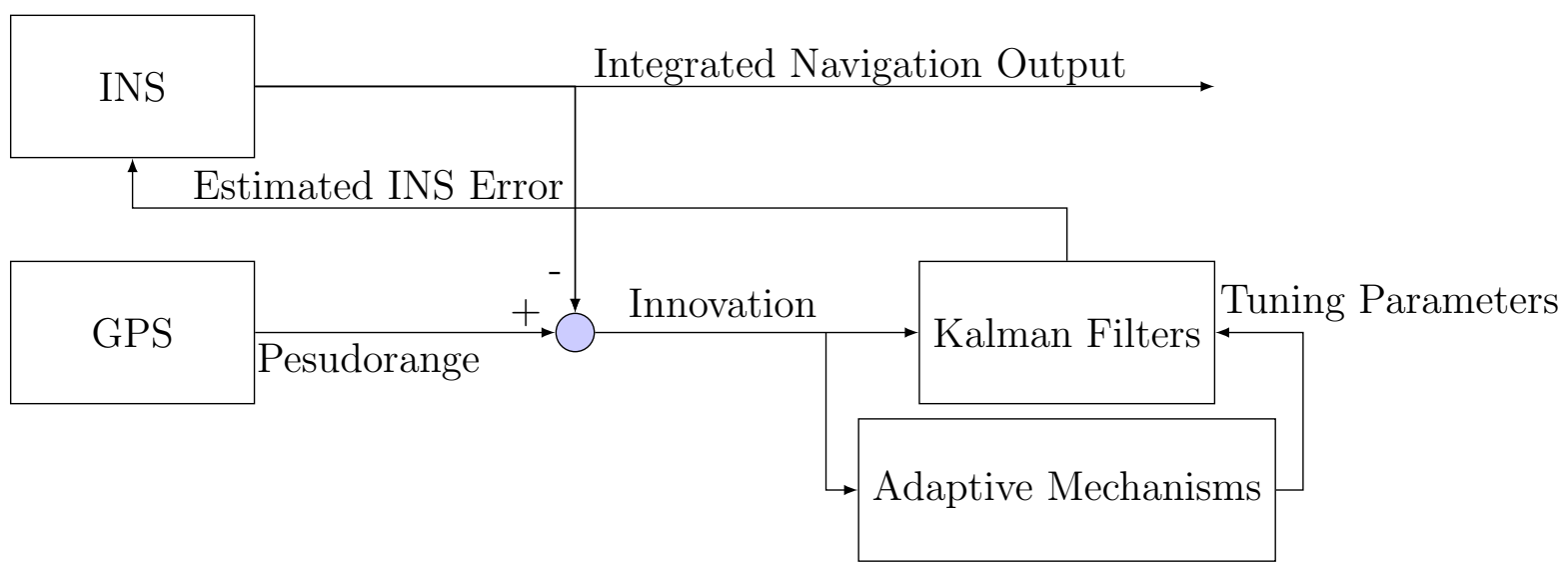

Figure. 5.8. Block diagram representation of adaptive Kalman filters for GPS/INS integration.

The reference trajectory is from the accumulation of inertial measurements through the strapdown algorithm. The adaptive Kalman filter's role is to estimate 
the deviation of the reference trajectory from the truth trajectory. This is then established the state vector. Hence, it is important to note that the state model for the Kalman filter here consists of inertial error quantities, rather than the total dynamical quantities. Correspondingly, the random-process model should reflect the random character of the errors in the inertial sensor. For the inertial error model, the chosen navigation reference frame will follow the locally level convention (see Appendix A.) where $\mathrm{x}$ points east, $\mathrm{y}$ points north, and $\mathrm{z}$ is the altitude above the reference WGS-84 ellipsoid. In the error models separate channels for the north, east, and altitude were considered [7]. For the simulation, initial conditions was selected based on examples in relevant literature [3] as follows:

$$
\begin{gathered}
\hat{x}_{0}=[0,0,0,0,0,0,0]^{T} \\
\boldsymbol{P}_{0}=\left[\begin{array}{cccccccc}
100 & 0 & 0 & 0 & 0 & 0 & 0 & 0 \\
0 & 10 & 0 & 0 & 0 & 0 & 0 & 0 \\
0 & 0 & 100 & 0 & 0 & 0 & 0 & 0 \\
0 & 0 & 0 & 10 & 0 & 0 & 0 & 0 \\
0 & 0 & 0 & 0 & 100 & 0 & 0 & 0 \\
0 & 0 & 0 & 0 & 0 & 10 & 0 & 0 \\
0 & 0 & 0 & 0 & 0 & 0 & 100 & 0 \\
0 & 0 & 0 & 0 & 0 & 0 & 0 & 10
\end{array}\right]
\end{gathered}
$$

The driving noise $\boldsymbol{w}$, was chosen as: 


$$
\boldsymbol{w}=\left[0, t \sigma_{x}, 0, t \sigma_{y}, 0, t \sigma_{z}, c S_{f} t+\frac{c S_{g} t^{2}}{2}, c S_{g} t\right]^{T}
$$

where $\sigma_{x}=0.0006, \sigma_{y}=0.0006$ and $\sigma_{z}=0.0006$ are INS standard deviations associated with $(x, y, z), t$ is the sample time, $S_{f}=0.4(10)^{-18}$ is the standard deviation of clock offset, and $S_{g}=1.58(10)^{-18}$ is the standard derivation associated with velocity. The process noise covariance defined as:

$$
\begin{aligned}
& \boldsymbol{Q}=E\left[\boldsymbol{w} \boldsymbol{w}^{T}\right] \\
& \boldsymbol{Q}=\left[\begin{array}{cccccccc}
\sigma_{x}^{2} t^{3} / 3 & \sigma_{x}^{2} t^{2} / 2 & 0 & 0 & 0 & 0 & 0 & 0 \\
\sigma_{x}^{2} t^{2} / 2 & \sigma_{x}^{2} t^{2} & 0 & 0 & 0 & 0 & 0 & 0 \\
0 & 0 & \sigma_{y}^{2} t^{3} / 3 & \sigma_{y}^{2} t^{2} / 2 & 0 & 0 & 0 & 0 \\
0 & 0 & \sigma_{y}^{2} t^{2} / 2 & \sigma_{y}^{2} t^{2} & 0 & 0 & 0 & 0 \\
0 & 0 & 0 & 0 & \sigma_{z}^{2} t^{3} / 3 & \sigma_{z}^{2} t^{2} / 2 & 0 & 0 \\
0 & 0 & 0 & 0 & \sigma_{z}^{2} t^{2} / 2 & \sigma_{z}^{2} t^{2} & 0 & 0 \\
0 & 0 & 0 & 0 & 0 & 0 & \left(S_{a} t+\frac{S_{b} t^{3}}{3}\right) c^{2} & \frac{S_{b} t^{2} c^{2}}{2} \\
0 & 0 & 0 & 0 & 0 & 0 & \frac{S_{b} t^{3} c^{2}}{2} & S_{b}
\end{array}\right]
\end{aligned}
$$

where $c$ is the speed of light, $S_{a}=0.5(10)^{-18}$ is the standard deviation of the clock offset and $S_{b}=1.68(10)^{-18}$ is the standard derivation associated with clock bias. The observation processed in the Kalman filter is the difference between the actual receiver measured pseudorange and predicted measurement based on the corrected 
INS position and corrected GPS receiver clock time. The Pseudorange equation is defined as follow:

$$
h_{j}=\sqrt{\left(X_{j}-x\right)^{2}+\left(Y_{j}-y\right)^{2}+\left(Z_{j}-z\right)^{2}}+c \triangle t_{j}
$$

where $\left(X_{j}, Y_{j}, Z_{j}\right)$ for $j=1,2, \ldots, 4$ representing the positions of the four GPS satellites, respectively. $(x, y, z)$ are the position of the vehicle, $\triangle t_{j}$ is a receiver offset from the $j_{t h}$ satellite. Noise with a standard deviation of 5 meters was considered for the GPS. The $\boldsymbol{R}$ matrix reflects the additive white measurement noise component, which in this model is defined as:

$$
\begin{gathered}
\boldsymbol{R}=E\left[\boldsymbol{v}^{T}\right] \\
\boldsymbol{R}=\left[\begin{array}{llll}
r & 0 & 0 & 0 \\
0 & r & 0 & 0 \\
0 & 0 & r & 0 \\
0 & 0 & 0 & r
\end{array}\right]
\end{gathered}
$$

where $r$ is the error variance, It should be mentioned that the initial conditions for all filters assumed the same. The AHEKF parameter $\gamma$ is designed based on trial and error, and to achieve minimum variance and high robustness, $\gamma$ was set to 1.53.

\subsubsection{Results}

Four different schemes to evaluate the performance of the proposed algorithm are considered to make an accurate comparison between different Kalman filter algorithms. 


\subsubsection{Scheme 1:Robustness Against High Initial Uncertain- ties}

In this case, performances of implemented filters affected by high initial uncertainties are considered. Here, the $\boldsymbol{R}_{k}$ and $\boldsymbol{Q}_{k}$ matrices in HEKF and UKF are assumed to be constant, while the $\boldsymbol{R}_{k}$ and $\boldsymbol{Q}_{k}$ matrices in AHEKF and FAUKF can be tuned. To evaluate the performance of the proposed AHEKF and FAUKF, all filters were tested for various parameter uncertainties. The effects of three different uncertainty values on the filter variances is presented in Figure. 5.9 - Figure. 5.11.
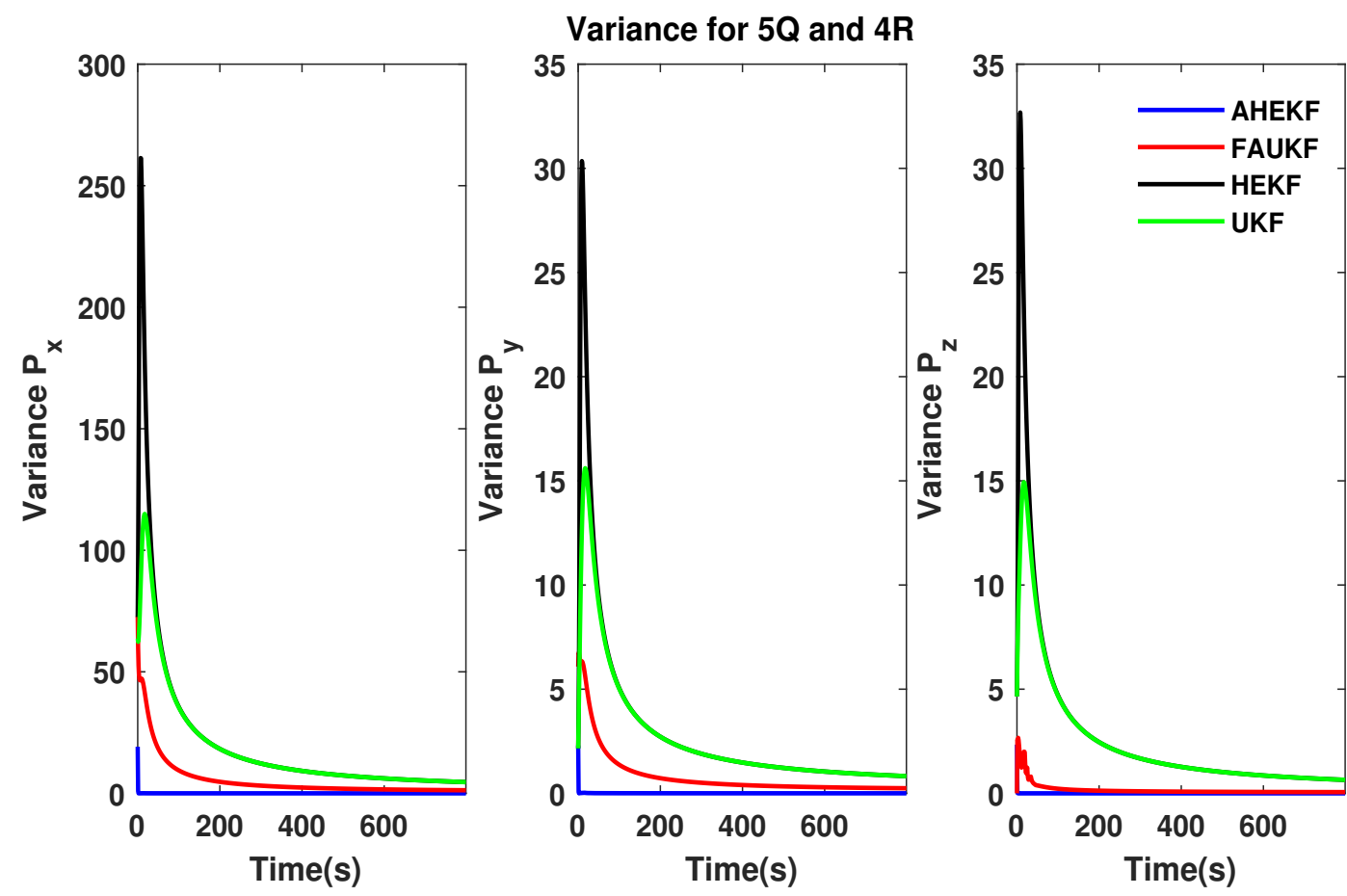

Figure. 5.9. Variance of the filters for $5 \mathrm{Q}$ and $4 \mathrm{R}$. 

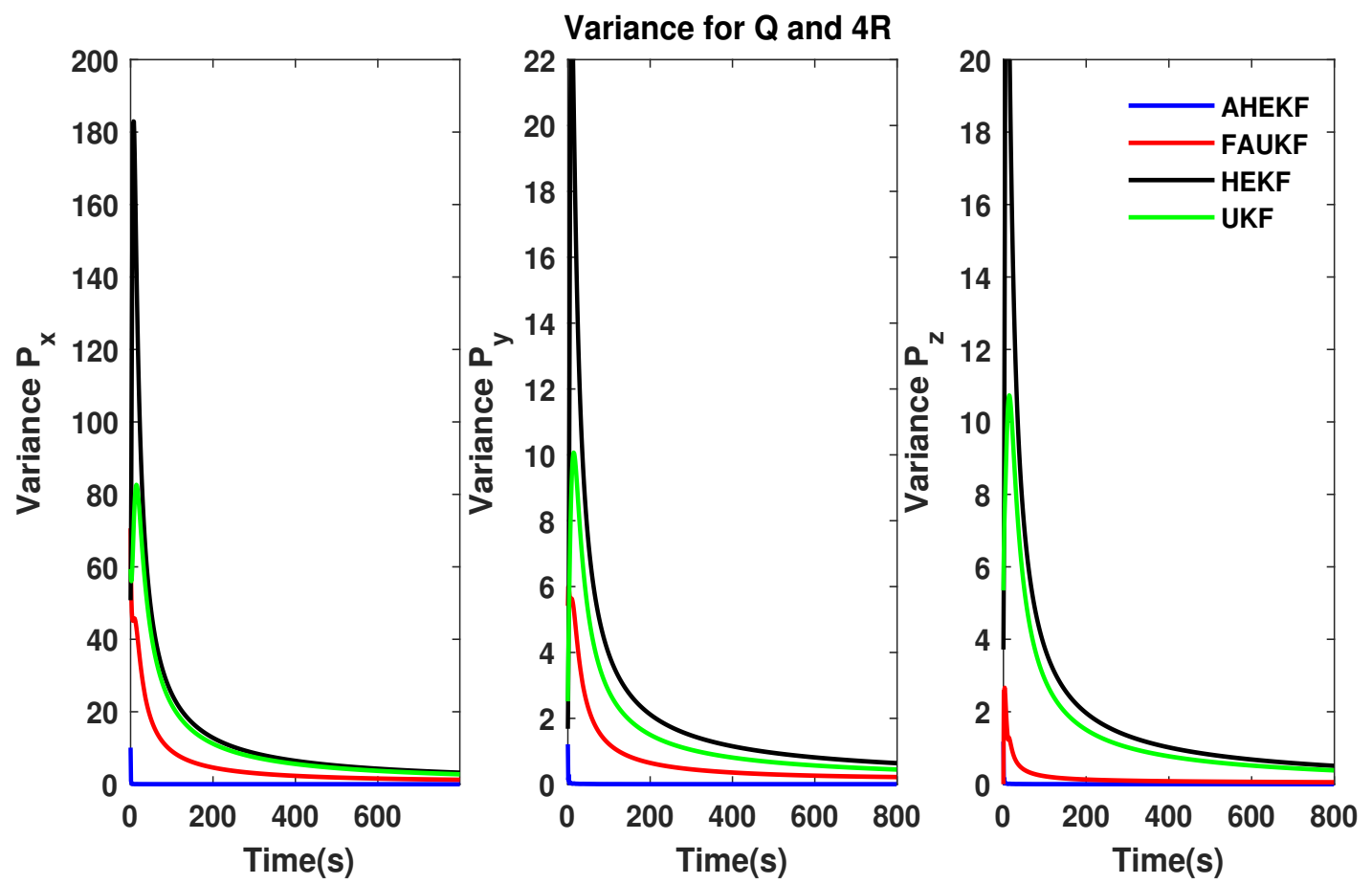

Figure. 5.10. Variance of the filters for $\mathrm{Q}$ and $4 \mathrm{R}$.
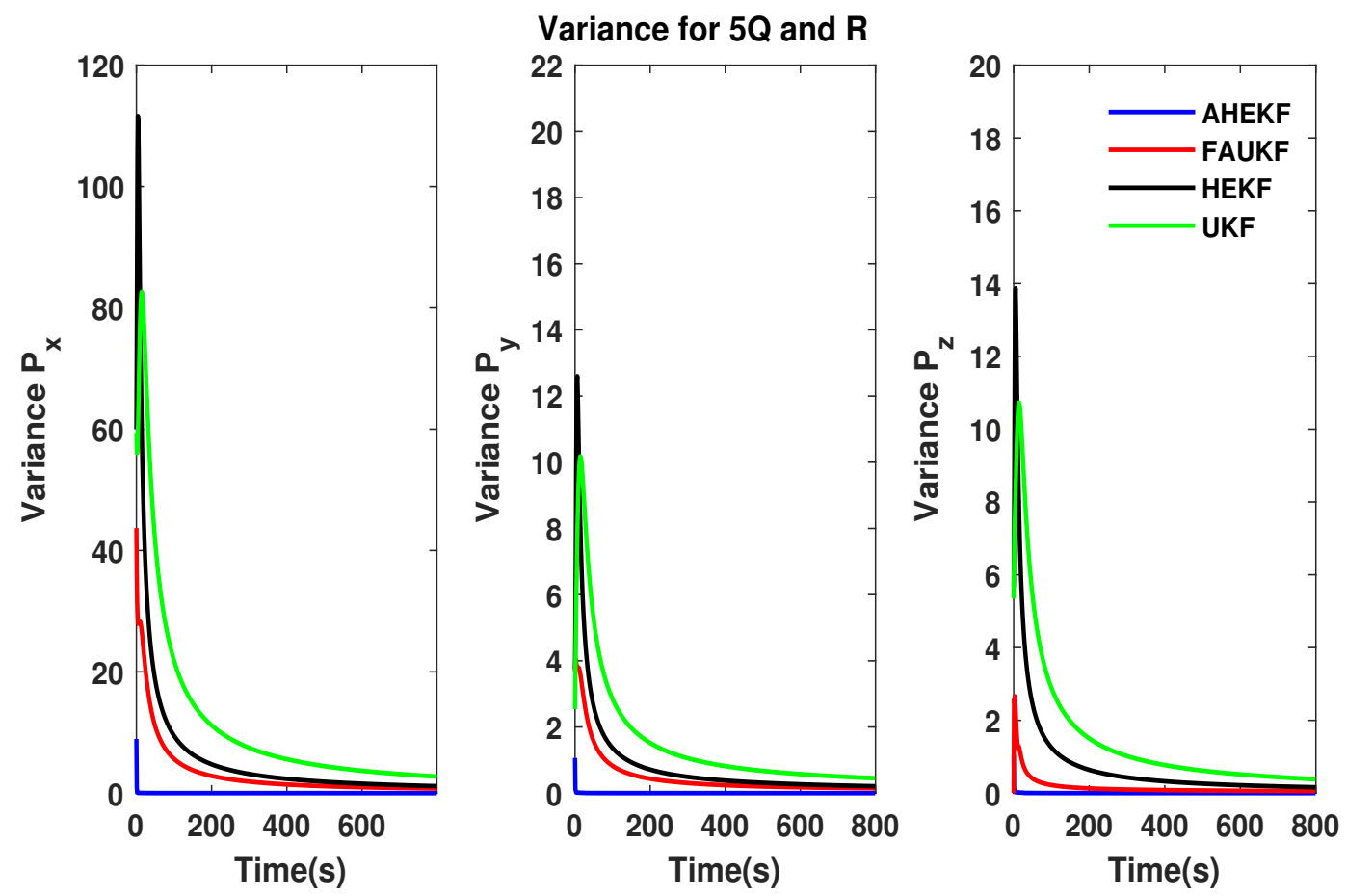

Figure. 5.11. Variance of the filters for $5 \mathrm{Q}$ and $\mathrm{R}$. 
Figure. 5.9 shows the variance in in meters squared of AHEKF, FAUKF, UKF and HEKF when high initial uncertainties $5 \boldsymbol{Q}$ and $4 \boldsymbol{R}$ exist. $5 \boldsymbol{Q}$ and $4 \boldsymbol{R}$ indicate that the real-time parameters are 5 and 4 times as large as the designed $\boldsymbol{Q}$ and $\boldsymbol{R}$. As shown, AHEKF has the best performance compared to the other filters. The variance converges to a value close to zero more quickly than the other filters. The simulation was repeated for different covariance values of $(\boldsymbol{Q}, 4 \boldsymbol{R})$ as shown in Figure. 5.10, and results for $(5 \boldsymbol{Q}, \boldsymbol{R})$ are illustrated in Figure. 5.11. Due to better treatment of parameter uncertainties, the AHEKF and FAUKF have shown performance improvement compared to conventional KFs and FAUKF and for all uncertainty values, AHEKF performed the best in managing high initial uncertainties.

\subsubsection{Scheme 2: Robustness Against Non-zero Mean Noise}

If the measurement and state noises are zero mean, uncorrelated and white noise the standard Kalman filters are the best solutions for state estimation. Based on [242] if the noises are white non-Gaussian, the standard Kalman filter are still the optimal solutions. The standard Kalman filter, however, assumes noise with zero-mean. To validate the performance of the proposed algorithms against non-zero mean noises, for the second part of this analysis, the performance of all filters is evaluated for different mean noise values. The experiment considers the effect of different INS noise mean values for the East (x), North (y), and Altitude (z) on the accuracy of the state estimate. The corrected position and velocity errors are shown in Figures. 5.12 - Figures. 5.15 for different noise mean values. The corrected error is defined as the current INS error minus the estimated INS error. Figures. 5.12 - Figures. 5.15 indicate the corrected position and velocity errors of AHEKF, FAUKF, HEKF, UKF and EKF when $\mu=0.1, \mu=0.5, \mu=1.5$ and $\mu=3$, respectively. It should be noted that $\mu$ is a coefficient of the INS standard deviation. As it shown, the proposed 
FAUKF and AHEKF improved the performance of the standard Kalman filters. The position and velocity errors of FAUKF, and FHEKF are much smaller than that of EKF, UKF, and HEKF, and as indicated, AHEKF outperforms the FAUKF, the position and velocity errors of the proposed AHEKF remain close to zero and stable at high mean values while the other state-of-the-art filters have large drifts. For a better comparison, the root mean square (RMS) for different mean values depicted in Figure. 5.16 - Figures. 5.19. As shown in Figure. 5.16, when $\mu$ is set to 0.1, the position and velocity errors in adaptive schemes are close to standard KFs, and when the mean increases the position and velocity errors of the AHEKF remain close to zero while the other filters diverge to high values. Figure. 5.17 represents the velocity and position RMS errors when $\mu$ is set to 1.5, and Figure. 5.18 and Figure. 5.19 indicate the position and velocity RMS errors for $\mu=1.5$ and $\mu=3$, respectively. From these results, we can conclude that, AHEKF performs better than other filters when dealing with non-zero mean noise caused by model uncertainty or biased measurements. 

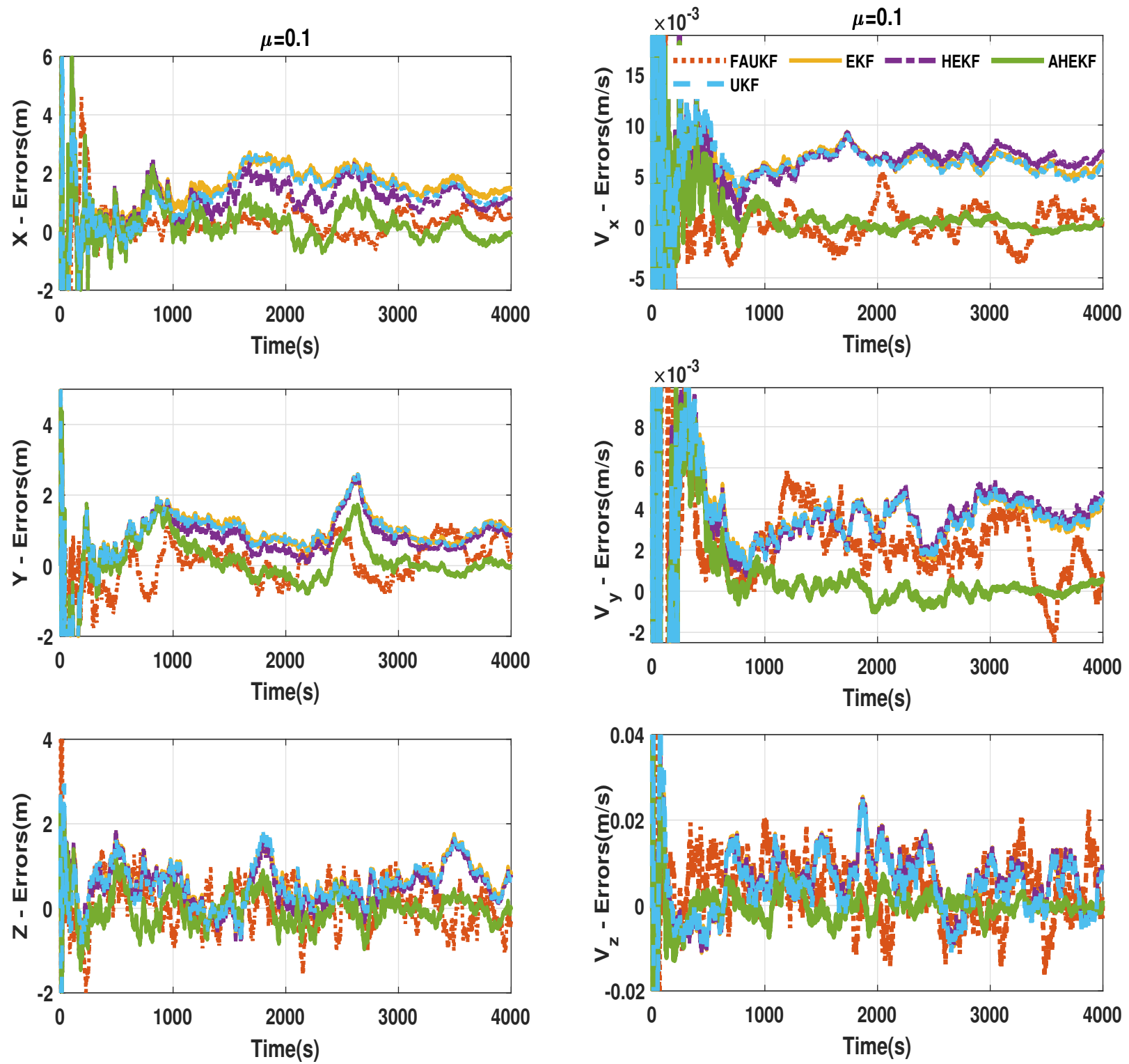

Figure. 5.12. Position and velocity errors comparison for $\mu=0.1$. 

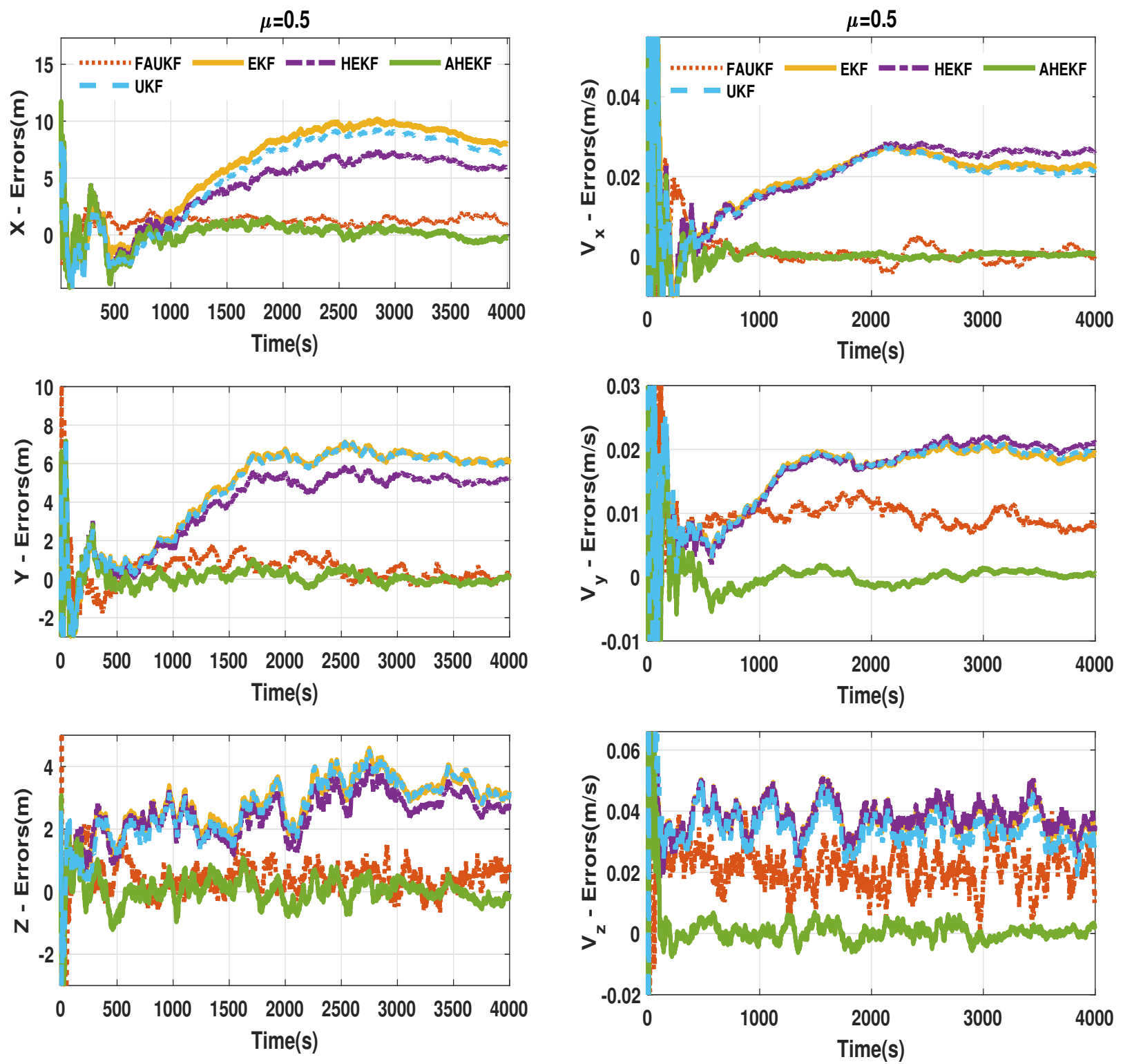

Figure. 5.13. Position and velocity errors comparison for $\mu=0.5$. 

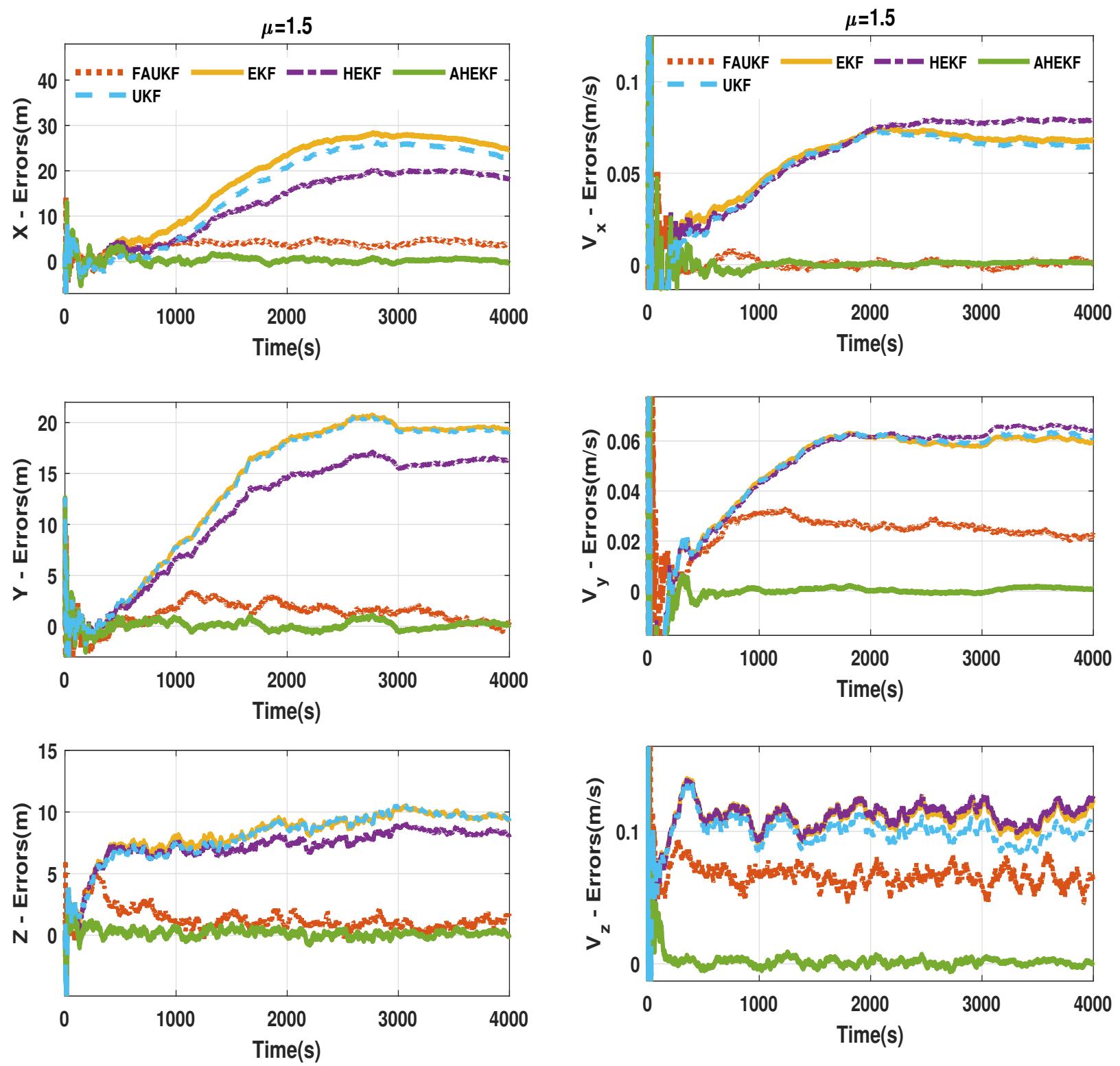

Figure. 5.14. Position and velocity errors comparison for $\mu=1.5$. 

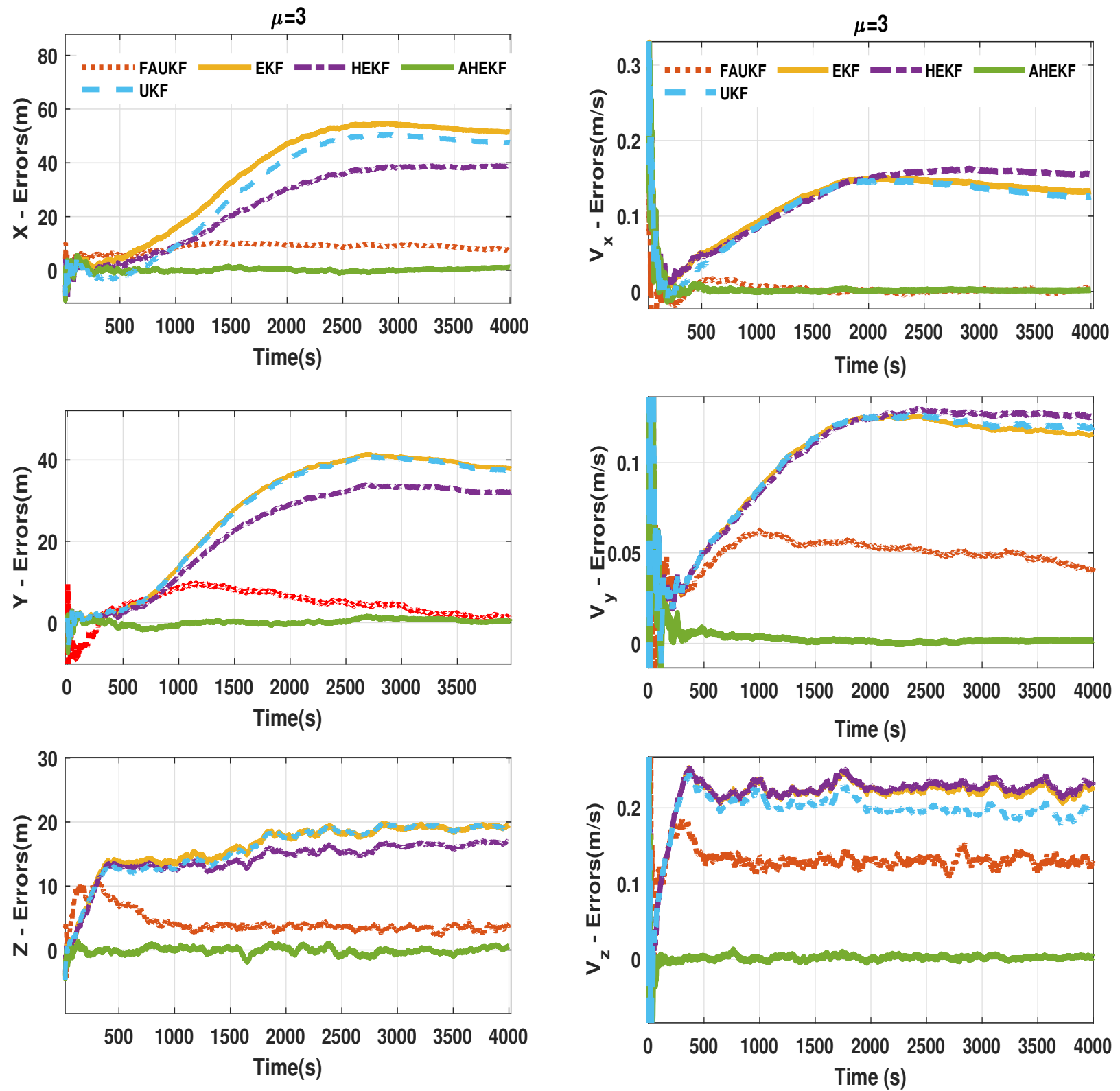

Figure. 5.15. Position and velocity errors comparison for $\mu=3$.

The innovation sequence is the difference between the best measurement prediction 

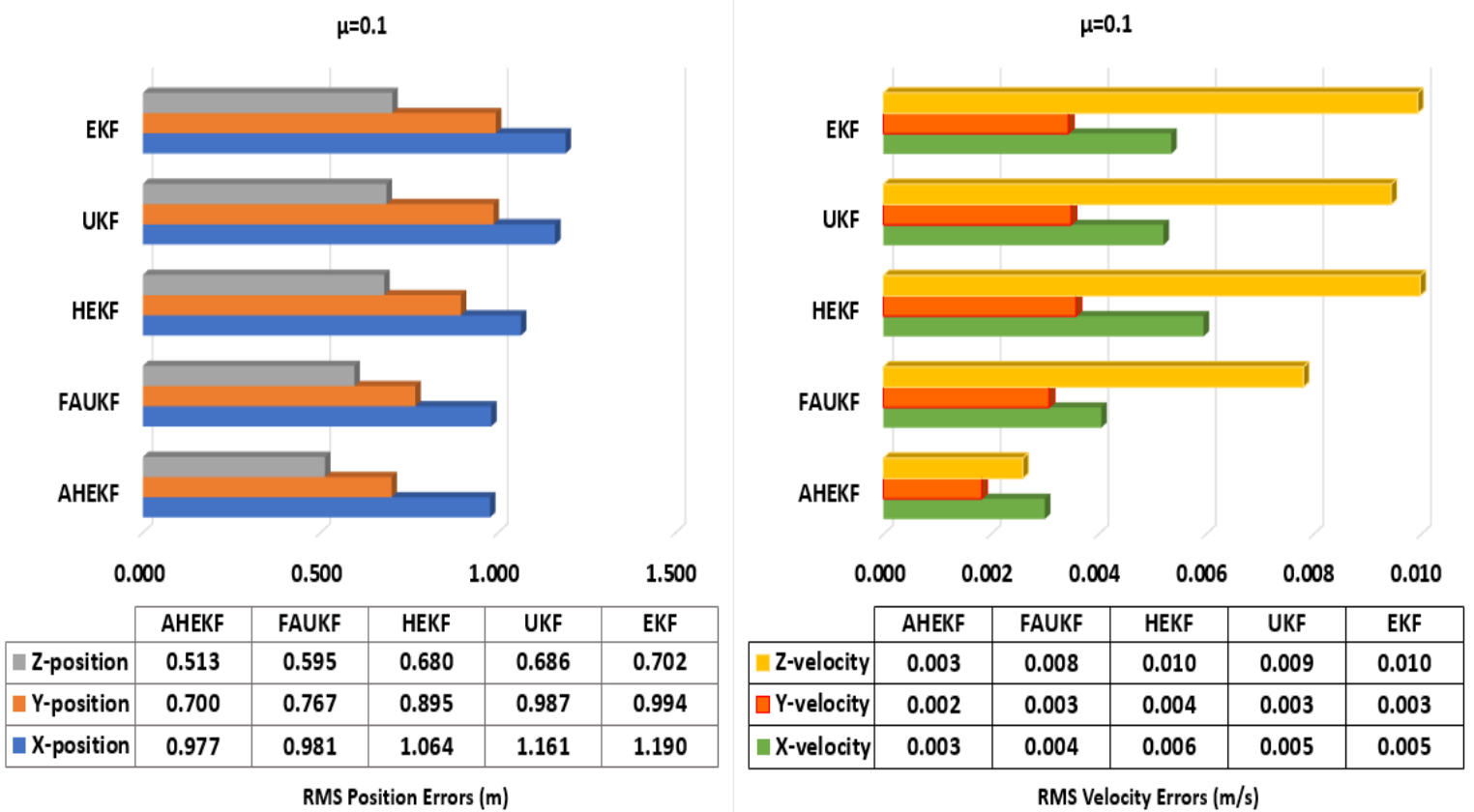

Figure. 5.16. Position and velocity RMS errors comparison for $\mu=0.1$.
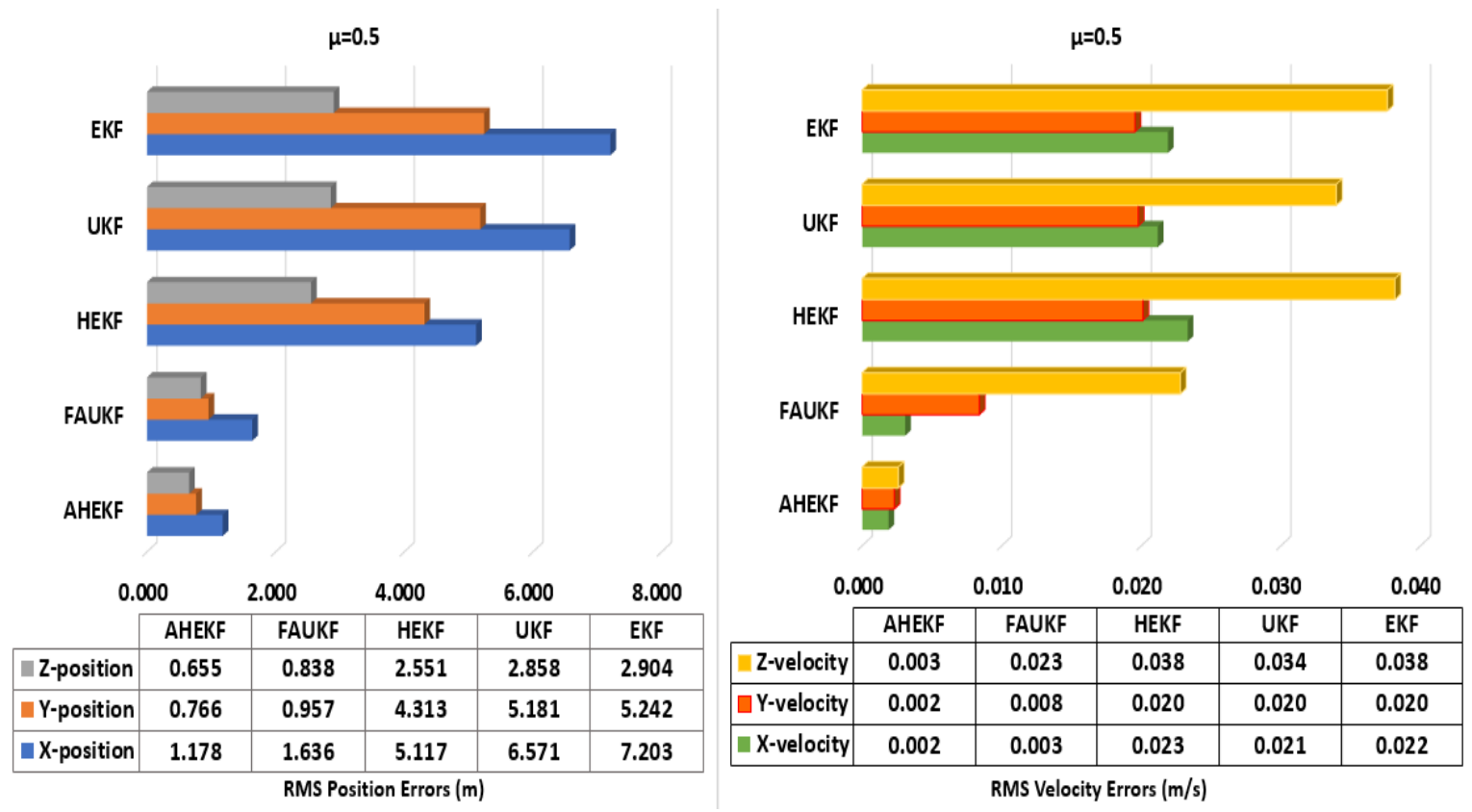

Figure. 5.17. Position and velocity RMS errors comparison for $\mu=0.5$. 

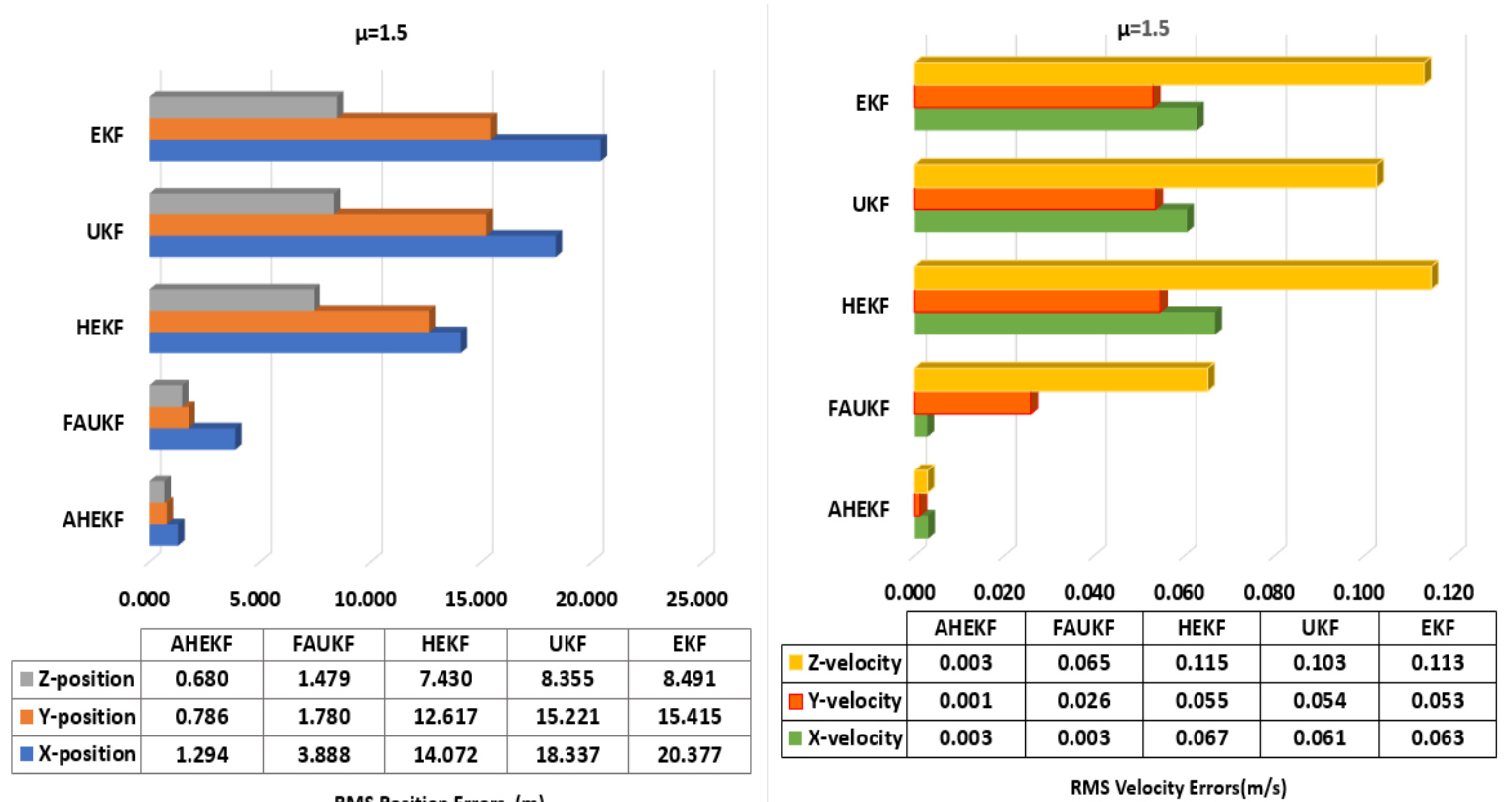

Figure. 5.18. Position and velocity RMS errors comparison for $\mu=1.5$.
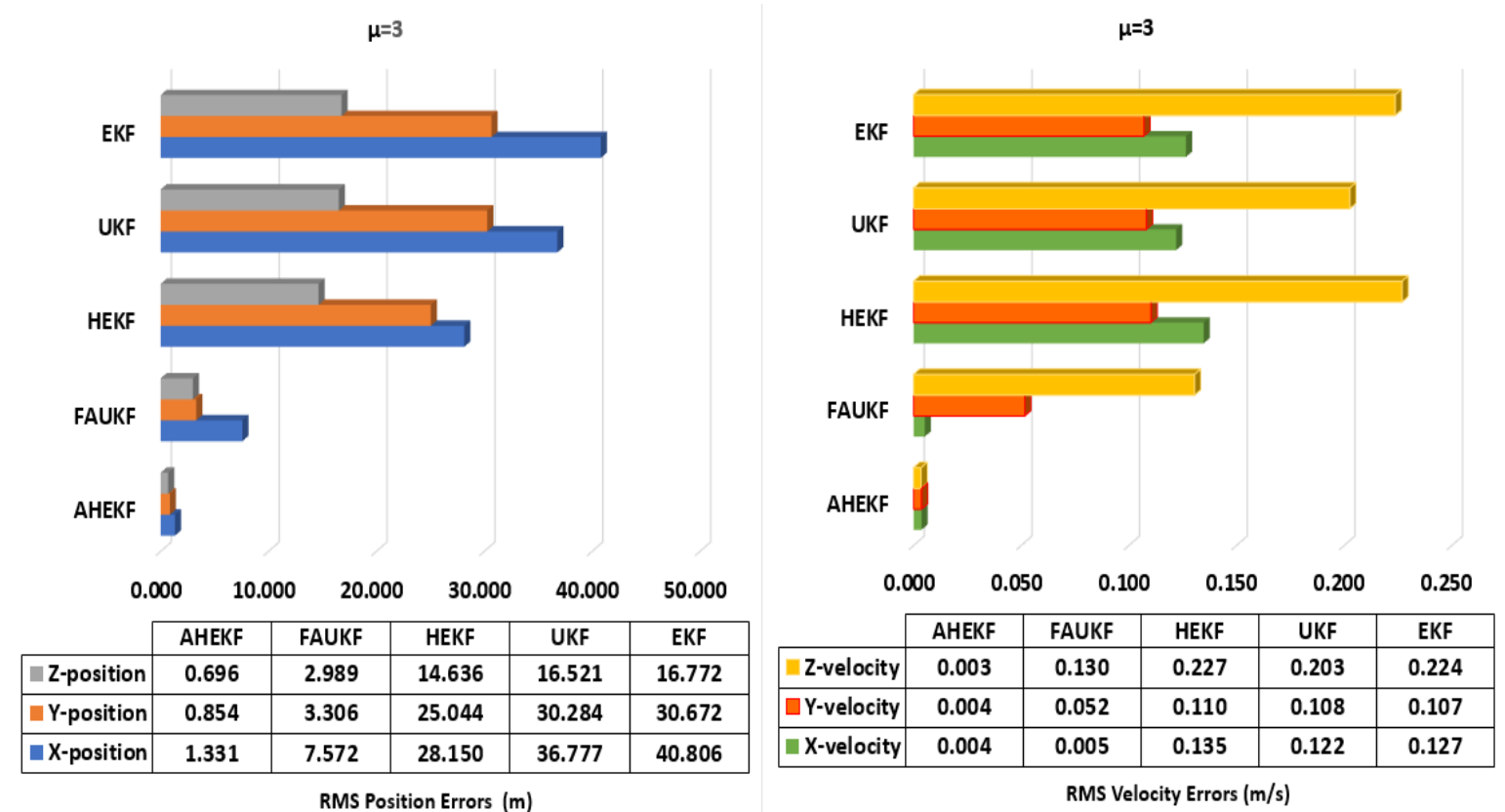

Figure. 5.19. Position and velocity RMS errors comparison for $\mu=3$. 
based on the filter's internal mode and the actual measurement; hence, it can be used to evaluate the filter's performance. Kalman filters are becoming unstable when the mean moves away from zero. The mean of the innovation sequence of filters for $\mu=0.5$ is shown in Figure. 5.20. From the figure, it is apparent that the innovation sequence of the EKF, UKF and HEKF have large drift, while the innovation mean values of the FAUKF and AHEKF are much smaller. The AHEKF has the best performance.

\subsubsection{Scheme 3: Robustness Against Non-zero Mean Noise and High Initial Uncertainties}

In this subsection, to evaluate the performance of the suggested approaches, both high-initial uncertainties and non-zero mean noise are assumed to exist simultaneously. Multiple cases have been evaluated with different coefficients of initial process and measurement covariance matrices and the various means of noise. Figure. 5.21 Figures. 5.26 illustrate the position and velocity RMS errors in the X, Y and Z directions individually. With position and velocity errors, it is evident that when $\mu=0.1$ the RMS error in all directions is minimal, while it increases significantly when we have $\left(5 \boldsymbol{Q}_{k}, 4 \boldsymbol{R}\right)$ and $\mu$ increases. A similar trend can be observed in velocity errors in all directions. The position errors of AHEKF and FAUKF are much smaller than those of EKF, UKF and HEKF. In all approaches, AHEKF demonstrated superior navigation accuracy performance than the others. It is evident that when the noise means increases, the RMS errors in both position and velocity increase. Besides, as shown in Figures. 5.21 and Figures. 5.26, AHEKF was less affected and maintained the RMS position and velocity errors of less than 3.4, 3.4 and 2 meters in the $X, Y$, and $Z$ directions, respectively while The RMS position errors of UKF peaked at 22.5, 


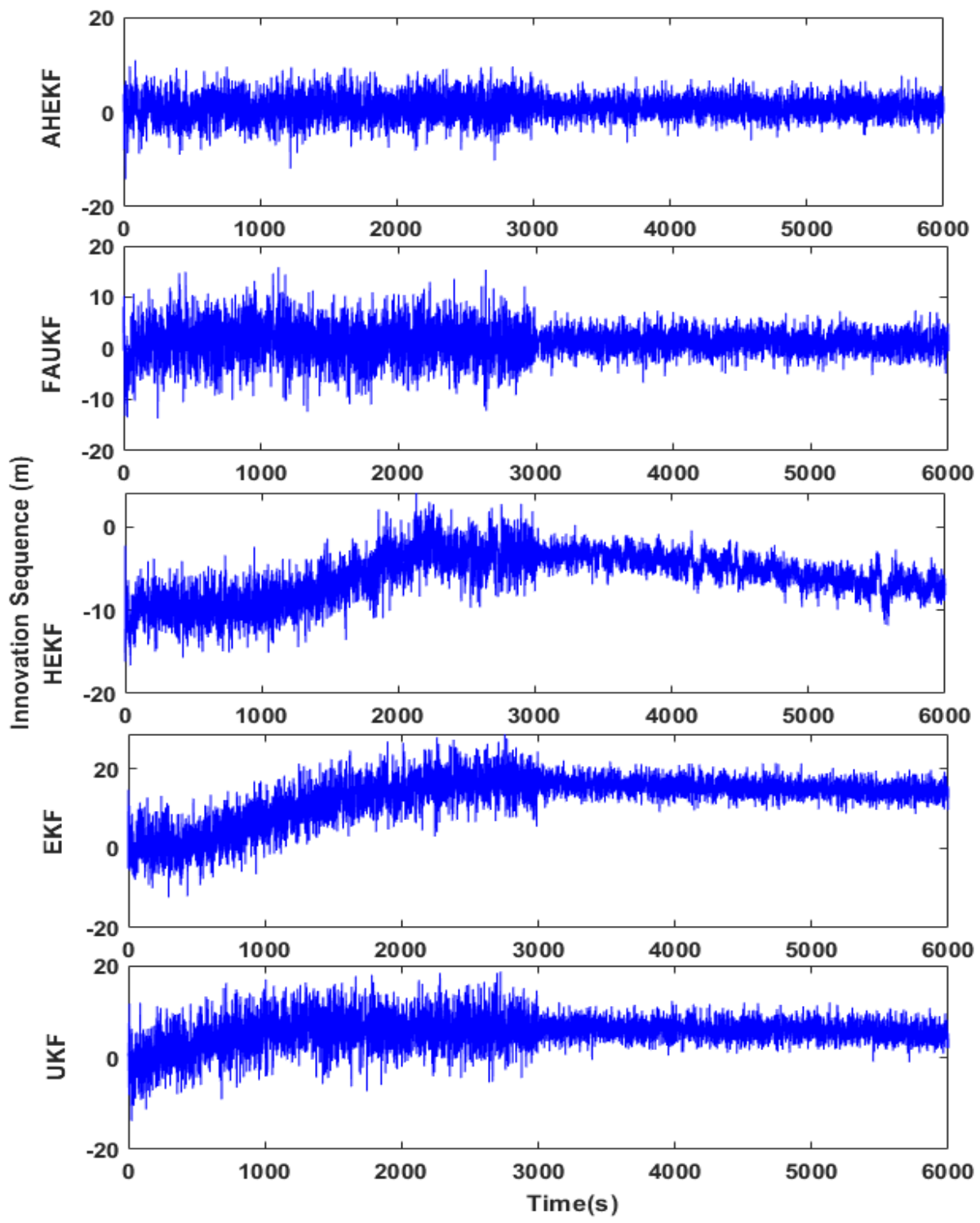

Figure. 5.20. Innovation sequences comparison. 


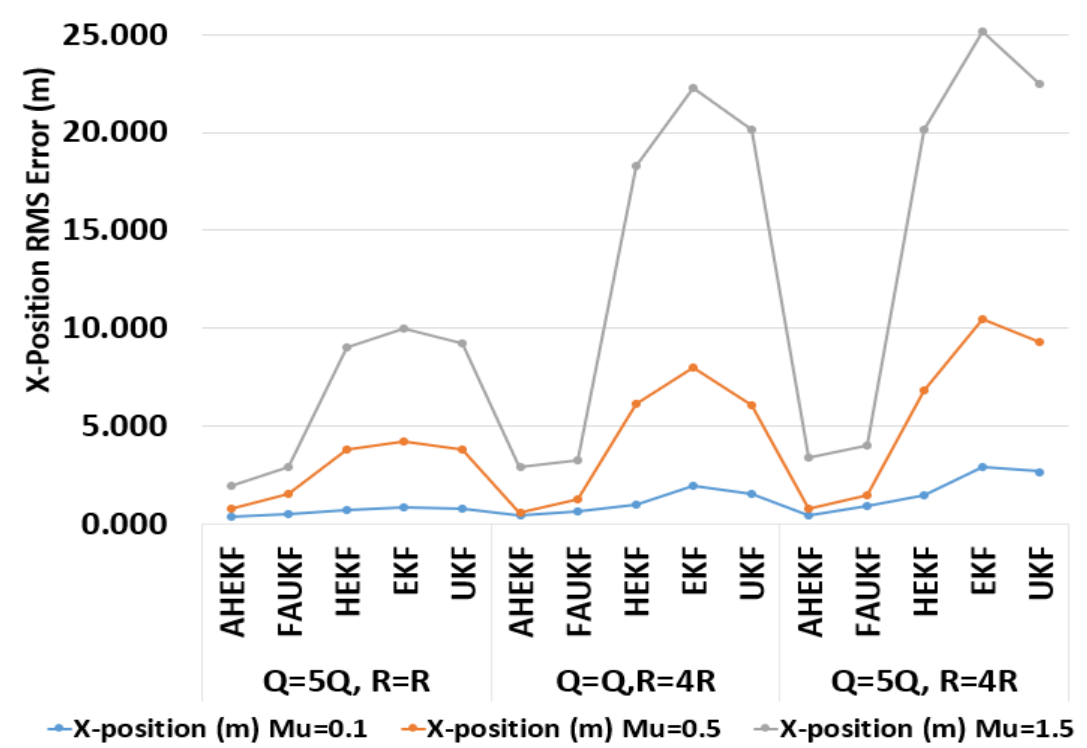

Figure. 5.21. Position RMS errors comparison in X-direction, when both non-zero means and high-initial uncertainties are applied.

18.1, 10 meters and EKF at 25, 19, 11 meters in $X, Y$ and $Z$ direction, respectively.

\subsubsection{Scheme 4: Robustness Against Measurement Outlier Data}

Outlier data can contaminate the measurement data due to GPS disturbances, such as GPS clock bias and drift. The outlier measurement data can affect the performance of the KFs. Here the performance of the filters is evaluated when outlier data exist in measurements. Figures. 5.27 and Figure. 5.28 illustrated the position and velocity error comparison between FAUKF, AHEKF, HEKF, and UKF when measurement outlier data was added artificially. As depicted, AHEKF shows better robustness 


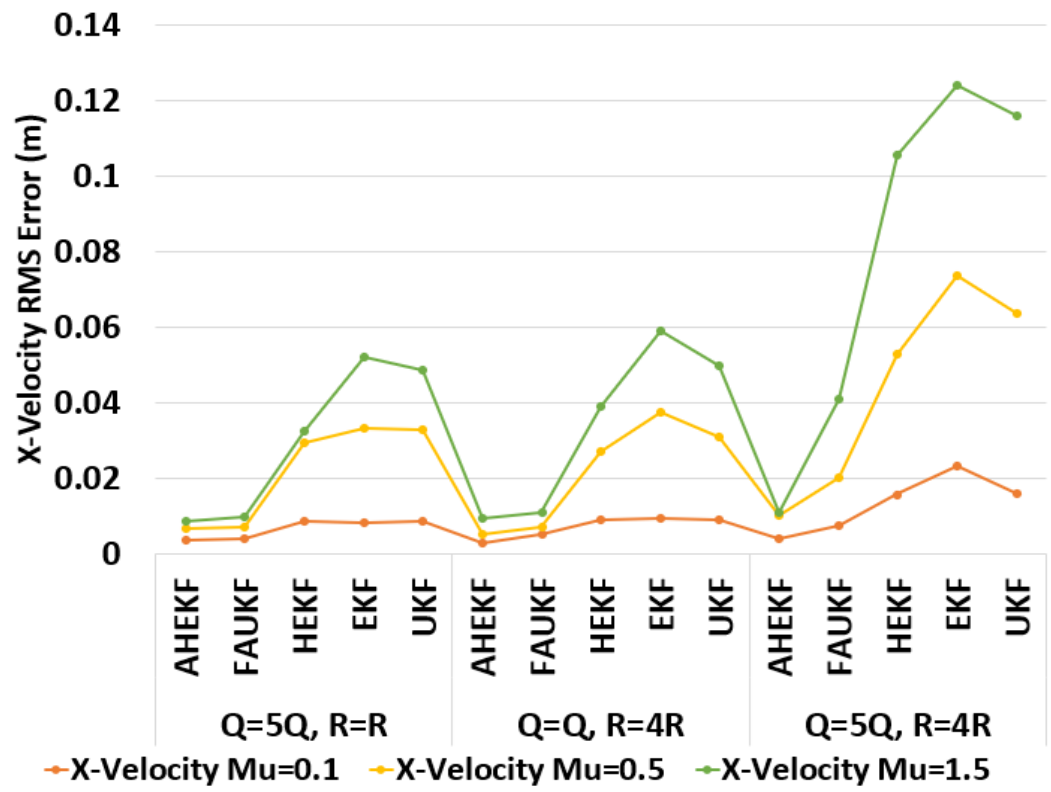

Figure. 5.22. Velocity RMS errors comparison in X-direction, when both non-zero means and high-initial uncertainties are applied.

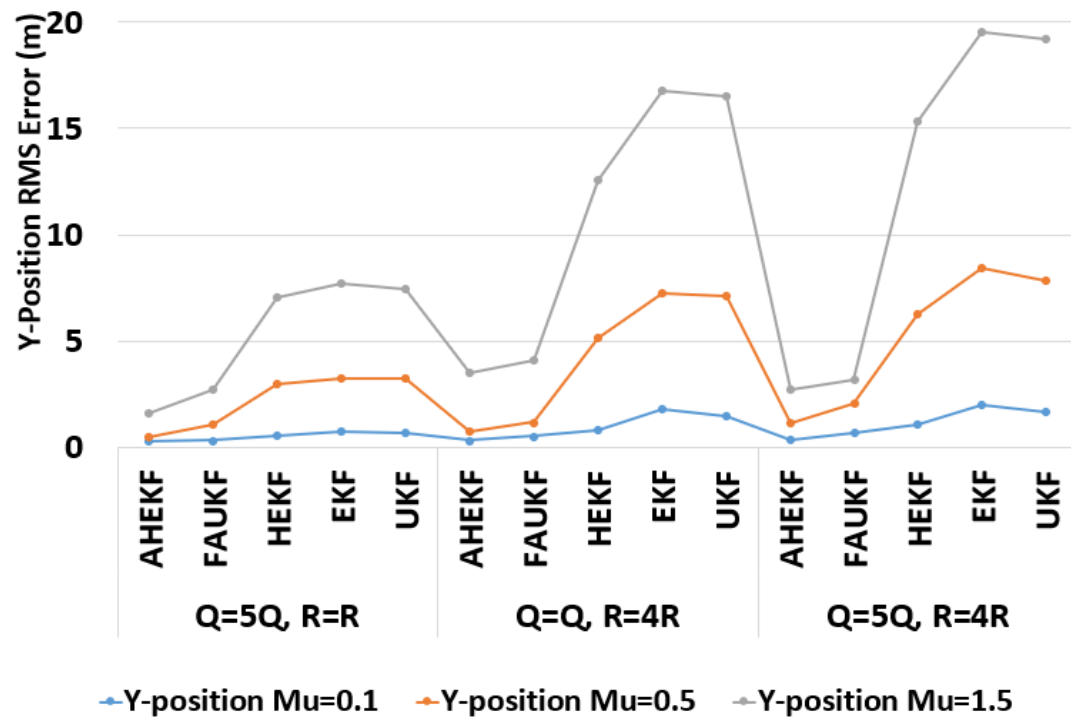

Figure. 5.23. Position RMS errors comparison in Y-direction, when both non-zero means and high-initial uncertainties are applied. 


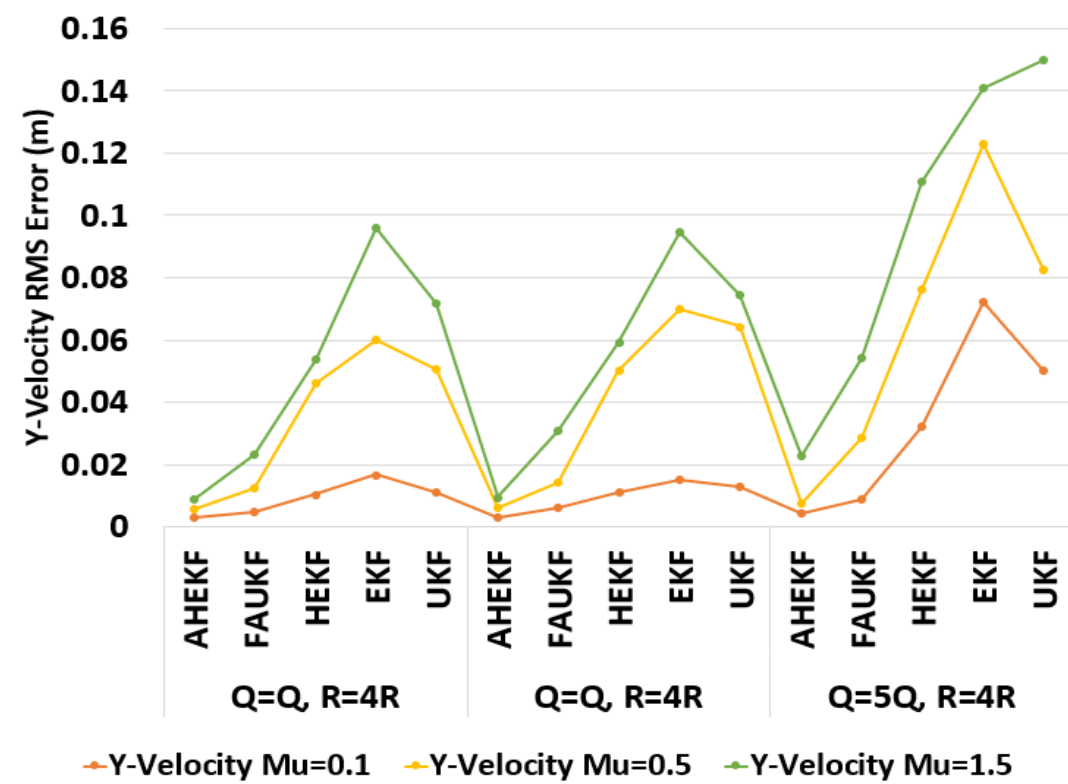

Figure. 5.24. Velocity RMS errors comparison in Y-direction, when both non-zero means and high-initial uncertainties are applied.

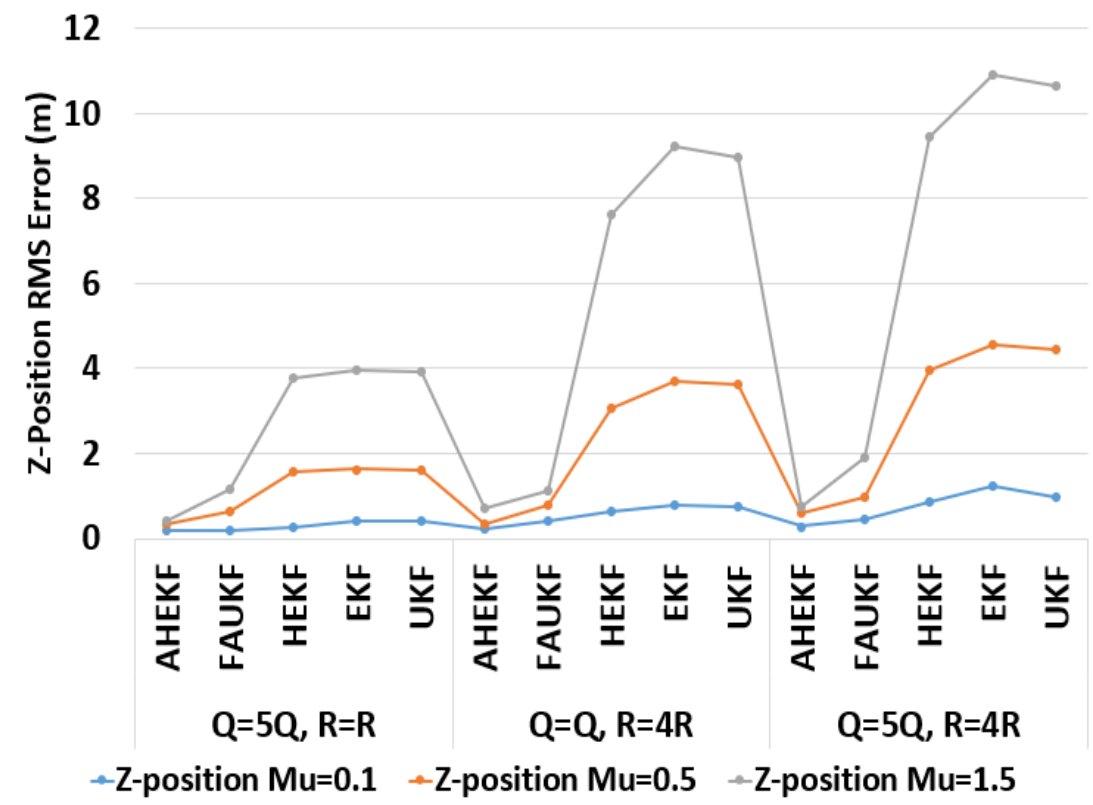

Figure. 5.25. Position RMS errors comparison in Z-direction, when both non-zero means and high-initial uncertainties are applied. 


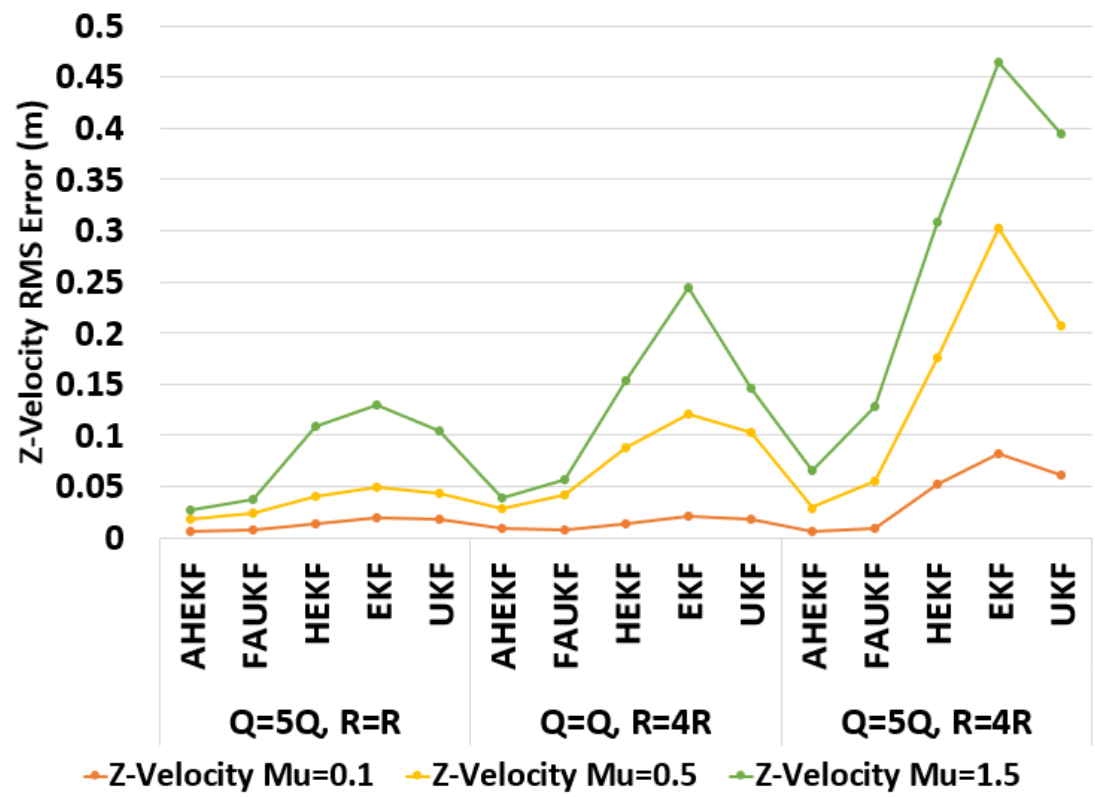

Figure. 5.26. Velocity RMS errors comparison in Z-direction, when both non-zero means and high-initial uncertainties are applied.

when outlier data added. The position and velocity errors also in the $X, Y$, and $Z$ directions decrease more than other Kalman filter structures when outlier data exist in the system. 

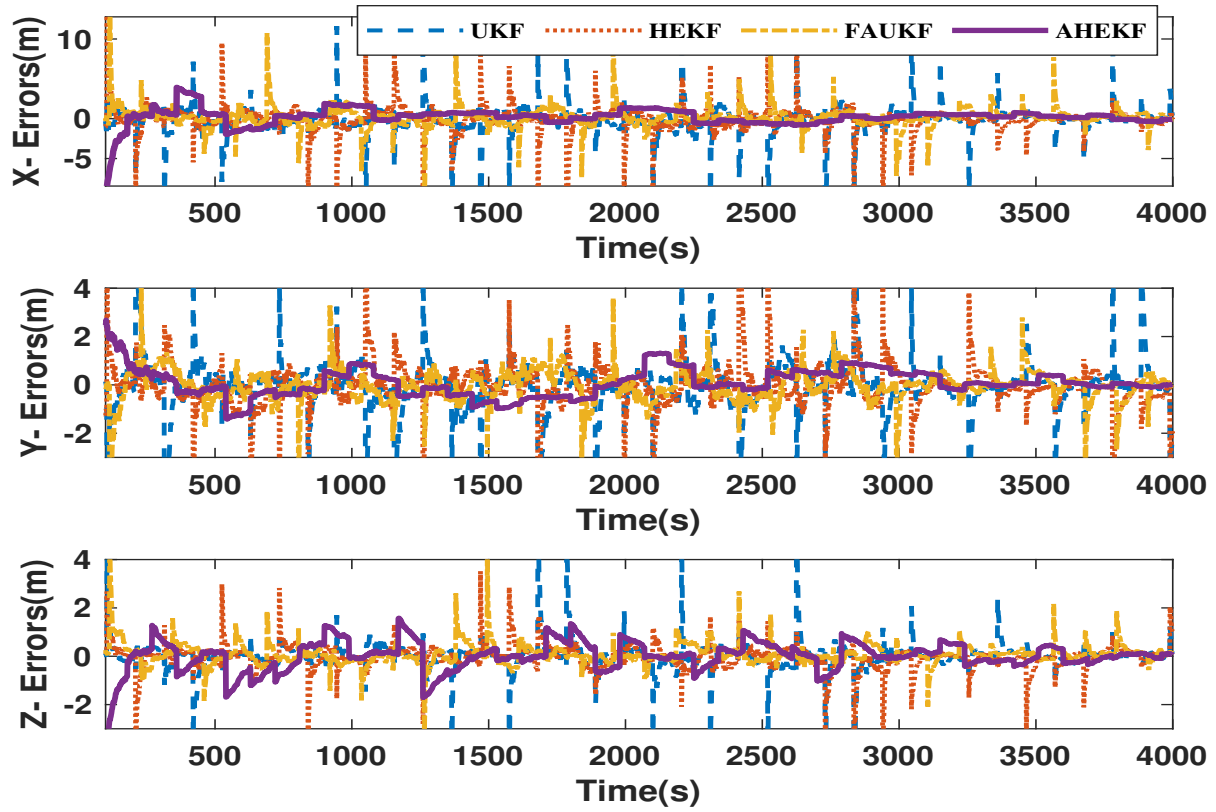

Figure. 5.27. Position errors comparison against outlier measurement data.
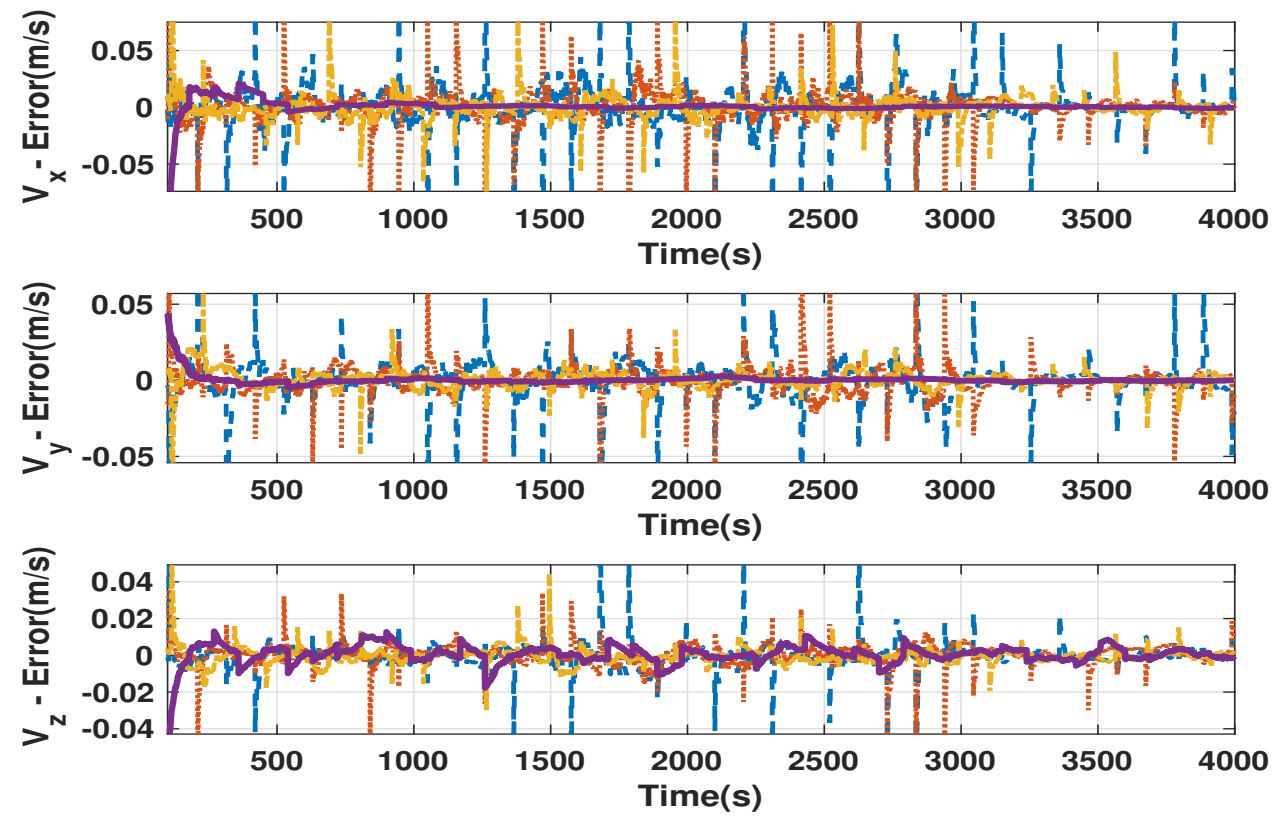

Figure. 5.28. Velocity errors comparison against outlier measurement data. 


\section{Chapter 6}

\section{Conclusion}

Due to human activity in recent decades, space is now congested with many different objects, including satellites and space debris. Many current and future in-orbit operations involve automated physical interactions with free-floating and free-flying targets. To achieve a full navigation solution for such operations, having precise details of a target's pose and motion is essential. However, this information is typically unavailable, particularly when damaged satellites, space debris and vibrating structures are involved. For space target pose and motion estimation, real-time tracking capabilities are a core component of many formation flights, including servicing satellites, autonomous rendezvous and docking, inspection, space debris removal and robotic maintenance [135]. Though the requirements for each application vary, robust, accurate and reasonably fast relative pose and motion estimation of the space target is essential. For this purpose, visual-based systems have several advantages, including low power usage, mass and volume requirements. For vision-based relative pose and motion estimation, target detection and tracking are the primary tasks of any formation flight, including missions to remove space debris. Detection and tracking of high-speed tumbling objects is very challenging, due to the lack of prior information about the target's structure and motion. To cope with uncertainty in 
real-time tracking of a non-cooperative space target, a new adaptation scheme of the Unscented Kalman filter is proposed in Chapter 2 of this work. The proposed approach employs a Fuzzy logic adaptation mechanism to detect divergence of UKF in order to implement an appropriate tuning parameter by monitoring the innovation sequence, thereby preventing the filter from becoming unstable. The proposed filter was employed to predict the location of a feature point in different video frames. The suggested method can manage some difficulties of maneuvering space targets, such as the disappearance of a feature point, complex space target motion and illumination changes. The actual space-based footage verified the robustness and effectiveness of the proposed tracking method, and performance comparisons of the Fuzzy Adaptive Unscented Kalman filter against EKF and UKF were conducted. The results revealed very promising potential enhancements to navigational robustness and accuracy, particularly in the case of occlusion.

Chapter 4 proposes a novel method to estimate the relative position, linear and angular velocities and attitude of a non-cooperative space target, based on visual measurements. The suggested approach employs a stereo camera to track a set of feature points on a target spacecraft, and UKF to perform the estimation procedure. The projection of tracked feature points from the two cameras creates an observation model of the filters, and the structure of a non-cooperative spacecraft is determined by estimating the feature points' positions. The relative attitude estimate is derived using the Modified Rodrigues Parameters. A number of simulation experiments were conducted to validate the accuracy and stability of this solution. The results demonstrate that the proposed algorithm can estimate the relative state between two spacecraft with satisfactory accuracy and guaranteed swift convergence. In addition, it can be concluded that the stereoscopic vision sensor yields promising results for estimating the relative state between two orbiting spacecraft, and can be used as a 
stand-alone sensor.

Due to the harsh space environment, different types of sensors are required, which means a practical sensor fusion algorithm is essential. Sensor fusion is a key aspect in the design of autonomous navigation, particularly algorithms that can accommodate sensor fusion techniques, enhance accuracy and resistant to malfunctions of individual sensors. Two novel adaptive schemes for data fusion are proposed in Chapter 5. The first scheme is based on a combination of Kalman filters and the Fuzzy logic controller that performs adaptation tasks for dynamic characteristics. Adaptive tuning of a Kalman filter with fuzzy logic is a promising strategy to cope with divergence when dealing with non-white noise and high initial uncertainty. Here, two Fuzzy logic adaptive mechanisms have been developed to improve UKF and IEKF performance by detecting uncertainly or time-varying parameters in both the process noise covariance matrix $\boldsymbol{Q}_{k}$, and the measurement covariance matrix $\boldsymbol{R}_{k}$. By monitoring the innovation sequences, the Fuzzy logic controllers continually adjust the noise strength in the filter's internal model and tune the filter. It is notable that the adaptive scheme developed for target tracking is different than the algorithm proposed for sensor fusion. For target tracking, one Fuzzy logic mechanism applies the appropriate tuning parameters and adapts the congenital UKF, while the sensor fusion framework uses two separate fuzzy logic controllers to manage the initial high uncertainty and white noise separately. Further, to improve performance the tuning parameter was optimized differently for both applications. The second scheme is a novel Fuzzy H-infinity Extended Kalman Filter (AHEKF). This approach benefits from using both robust and adaptive algorithms to overcome high uncertainties and noises, while maintaining a suitable estimation accuracy. Two adaptive sections are considered: First, an adaptive scale factor is designed to make the filter capable of controlling the effect of non-zero mean noises. Secondly, an adaption of $\boldsymbol{R}_{k}$ and $\boldsymbol{Q}_{k}$ weights are considered to 
control the effect of high initial uncertainties and outliers. Two adaptive approaches are applied to fuse signals from GPS and INS for autonomous vehicles. These two sensors were chosen as GPS/INS are using in many spacecraft proximity operations. To evaluate the performances of the proposed algorithms, three different schemes are assumed to involve robustness against high initial uncertainty, non-zero mean noises and outlier data. In the first scheme, the covariance of position and velocity are compared, and the results prove that when various high initial conditions are considered, the AHEKF performance is much better in terms of the fastest convergence and least estimation errors than other filters. The second scheme compares the proposed algorithm against the non-zero mean noises. Evaluations indicate that when the non-zero mean increases, not only does the AHEKF perform with more robust efficiency, but it also has fewer estimation errors in position and velocity. The third scheme involves evaluation of the worst-case initial condition, in which both high uncertainties and non-zero mean are considered. The results of FAUKF and AHEKF were compared to the FAEKF, UKF, HEKF and EKF. As the simulation results have shown, the AHEKF filter has the best performance in estimation accuracy, and high potential to be an alternative navigation state estimator. 


\section{List of References}

[1] J. Sasiadek and Q. Wang, "Low cost automation using ins/gps data fusion for accurate positioning," Robotica, vol. 21, no. 3, pp. 255-260, 2003.

[2] S. Yazdkhasti and J. Z. Sasiadek, "Multi sensor fusion based on adaptive kalman filtering," in Advances in Aerospace Guidance, Navigation and Control, pp. 317333, Springer, 2018.

[3] Q. Wang, Control and navigation system for autonomous vehicles and robots using fuzzy logic and Kalman filtering. PhD thesis, Carleton University, 1999.

[4] S. Ruel, T. Luu, and A. Berube, "On-orbit testing of target-less tridar 3d rendezvous and docking sensor," in Proc of the International Symposium on Artificial Intelligent, Robotics and Automation in Space (i-SAIRAS 2010), 2010.

[5] J. O'Sullivan, "Sts-131," in Japanese Missions to the International Space Station, pp. 159-172, Springer, 2019.

[6] S. Yazdkhasti and J. Z. Sasiadek, "Space robot relative navigation for debris removal," IFAC-PapersOnLine, vol. 50, no. 1, pp. 7929-7934, 2017.

[7] R. G. Brown and P. Y. Hwang, Introduction to random signals and applied Kalman filtering: with MATLAB exercises. J Wiley \& Sons, 2012.

[8] B. R. Sullivan and D. L. Akin, A survey of serviceable spacecraft failures. American Institute of Aeronautics and Astronautics, 2001.

[9] B. Weeden, "Overview of the legal and policy challenges of orbital debris removal," in Space Policy, pp. 38-43, 2011.

[10] V. J. Dubanchet, D. Saussié, D. Alazard, C. Bérard, and C. Peuvédic, Modeling and control of a space robot for active debris removal. CEAS Space Journal, 2014. 
[11] A. Gorman, "Space junk," An Insider's Guide to a Rapidly Changing Planet, p. 239.

[12] K. Bonn, "Active removal of space debris-discussing technical and economical issues," in Johnson Space Center(IAC), pp. 11-19, 2006.

[13] J. Liou, N. Johnson, and N. Hill, "Controlling the growth of future leo debris populations with active debris removal," in Acta Astronautica, p. 648-653, 2010.

[14] E. Bornschlegl, G. Hirzinger, M. Maurette, R. Mugnuolo, and G. Visentin, "Space robotics in europe, a compendium," in Proceedings of the 7th International Symposium on Artificial Intelligence, Robotics, and Automation in Space: i-SAIRAS, (NARA, Japan), 2003.

[15] J. C. Piedboeuf and E. Dupuis, "Recent canadian activities in space automation and robotics - an overview," in Proceedings of the 7th International Symposium on Artificial Intelligence, Robotics, and Automation in Space: i-SAIRAS, (NARA, Japan), 2003.

[16] S. Mohiuddin and M. . Psiaki, "High-altitude satellite relative navigation using carrier-phase differential global positioning system techniques," Guidance, Control, and Dynamics, vol. 30, pp. 1427-1436, 2007.

[17] S. Mohiuddin and M. . Psiaki, "Carrier-phase differential global positioning system navigation filter for high-altitude spacecraft," Guidance, Control, and Dynamics, vol. 31, pp. 801-814, 2008.

[18] I. Kawano, M. Mokuno, T. Kassai, and T. Suzuki, "Results and evaluation of autonomous rendezvous docking experiments of etsvii," in AIAA Guidance, Navigation, and Control, (Oregon), p. 1-10, 1999.

[19] G. Purcell, D. Kuang, S. Lichten, C. Wu, and L. Young, "Autonomous formation flyer (aff) sensor technology development," in AAS Guidance and Control Conference, 1998.

[20] J. Miller and J. Parker, "Nasa gnss activities," in Twelfth meeting of the International Committee on Global Navigation Satellite Systems, Working Group B, 2017. 
[21] F. H. Bauer, J. J. Parker, B. Welch, and W. Enderle, "Developing a robust, interoperable gnss space service volume (ssv) for the global space user community," in Proceedings of the 2017 International Technical Meeting of The Institute of Navigation, pp. 132-149, 2017.

[22] D. Marareskul, "Space service volume and russian geo satellites pnt," in United Nations International Committee on GNSS, Seventeenth Meeting of the Providers' Forum, Sochi, Russia, 2016.

[23] B. Ashman, F. H. Bauer, J. Parker, and J. Donaldson, "Gps operations in high earth orbit: Recent experiences and future opportunities," in 2018 SpaceOps Conference, p. 2568, 2018.

[24] R. Howard, A. Heaton, R. Pinson, and C. Carrington, "Orbital express advanced video guidance sensor," in IEEE Aerospace Conference, p. 1-10, 2008.

[25] H. Yano, T. Kubota, H. Miyamoto, T. Okada, D. Scheeres, Y. Takagi, K. Yoshida, M. Abe, S. Abe, O. Barnouin-Jha, A. Fujiwara, S. Hasegawa, T. Hashimoto, M. Ishiguro, M. Kato, J. Kawaguchi, T. Mukai, J. Saito, S. Sasaki, and M. Yoshikawa, "Touchdown of the hayabusa spacecraft at the muses sea on itokawa," in Science, 312(5778), p. 1350-1353, 2006.

[26] S. Ruel, T. Luu, and A. Berube, "Space shuttle testing of the tridar 3d rendezvous and docking sensor," Field Robotics, vol. 29, p. 535- 553, 2012.

[27] B. Naasz, D. Zimpfer, R. Barrington, and T. Mulder, "Flight dynamics and gnc for spacecraft servicing missions," in AIAA Guidance, Navigation and Control Conference, (Toronto, Canada), pp. 1-16, 2010.

[28] S. G. MacLean and H. L. Pinkney, "Machine vision in space," Canadian Aeronautics and Space Journal, vol. 29, p. 63-77, 1993.

[29] T. Yamamoto, N. Murakami, Y. Nakajima, and K. Yamanaka, "Navigation trajectory design for japanese active debris removal mission," in 24th International Symposium on Space Flight Dynamics (ISSFD), (Laurel, Maryland), 2014.

[30] F. Terui, H. Kamimura, and S. Nishida, "Motion estimation to a failed satellite on orbit using stereo vision and 3d model matching," in 9th International Conference on Control, Automation, Robotics and Vision, pp. 1-8, 2006. 
[31] T. Fuyuto, "Model based visual relative motion estimation and control of a spacecraft utilizing computer graphics," in 21st International Symposium on Space Flight dynamics, (Tolouse, France), 2009.

[32] C. Miravet, L. Pascual, E. Krouch, and J. Delcura., "An image-based sensor system for autonmous rendezvous with uncooperative satellites," in 7th International ESA Conference on Guidance, Navigation and Control Systems, 2008.

[33] F. Aghili and K. Parsa, "An adaptive vision system for guidance of a robotic manipulator to capture a tumbling satellite with unknown dynamics," in IEEE/RSJ International Conference on Intelligence Robots and Systems, p. 3064-3071, 2008.

[34] S. Segal, A. Carmi, and P. Gurfil, "relative state estimation of non-cooperative spacecraft under modeling uncertainity," in IEEE Aerospace Conference, pp. 18, 2011.

[35] S. Persson, B. Jacobsson, and E. Gill, "Prisma- demonstration mission for advanced rendezvous and formation flying technologies and sensors," in 56th International Astronautical Congress), 2005.

[36] D. Reintsema, J. Thaeter, A. Rathke, W. Naumann, P. Rank, and J. . Sommer, "Deos-the german robotics approach to secure and de-orbit malfunctioned satellites from low earth orbits," in In Proceedings of the i-SAIRAS), pp. 244$251,2010$.

[37] P. Lamy, L. Damé, S. Vivès, and A. Zhukov, "Aspiics: a giant coronagraph for the esa/proba-3 formation flying mission," in International Society for Optics and Photonics, pp. 773118-773118, 2010.

[38] S. Augenstein and S. Rock, "Simultaneous estimation of target pose and 3d shape using the fastslam algorithm," in AIAA Guidance, Navigation and Control Conference, (Chicago, Illinois, USA), pp. 497-513, 2009.

[39] A. Sonnenburg, M. Tkocz, and K. Janschek, "Ekf-slam based ap proach for spacecraft rendezvous navigation with unknown target spacecraft," in 18th IFAC Symposium on Automatic Control in Aerospace, (Nara, Japan), September 2010 . 
[40] S. Augenstein and S. Rock, "Improved frame-to-frame pose tracking during vision-only slam/sfm with a tumbling target," in IEEE International Conference on Robotics and Automation (ICRA), pp. 3131-3138, 2011.

[41] X. Du, B. Liang, W. Xu, and Y. Qiu, "Pose measurementoflargenoncooperativesatellitebasedon collaborativecameras," in Acta Astronautica, vol. 68, pp. 2047-2065, 2011.

[42] N. Oumer and G. Panin, "Tracking and pose estimation of non-cooperative satellite for on-orbit servicing," in International Symposium on Artificial Intelligence, Robotics and Automation, 2012.

[43] M. Lichter and S. Dubowsky, "Estimation of state, shape, and inertial parameters of space objects from sequences of range images," in Intelligent Robots and Computer Vision XXI:Algorithms Techniques, and Active Vision, vol. 52, p. 194-205, 2003.

[44] M. Abraham, P. Jasiobedzki, and M. Umasuthan, "Robust 3d vision for autonomous space robotic operation,", in Proceedings of the 6th International Symposium on Artificial Intelligence and Robotics and Automation in Space: i-SAIRAS, pp. 18-22, 2001.

[45] P. Jasiobedzki, M. Greenspan, and G. Roth, "Pose determination and tracking for autonomous satellite capture," in Proceedings of the 6th International Symposium on Artificial Intelligence and Robotics and Automation in Space: i-SAIRAS, pp. 48-53, 2001.

[46] B. E. Tweddle, "Computer vision-based localization and mapping of an unknown, uncooperative and spinning target for spacecraft proximity operations," in PhD Thesis, (Massachusetts Institute of Technology), 2013.

[47] G. Young and R. Chellappa, "3-d motion estimation using a sequence of noisy stereo images: models, estimation, and uniqueness results," in IEEE Transactions on Pattern Analysis and Machine Intelligence, vol. 12, pp. 735 - 759, 1990.

[48] C. Long, G. Bao-long, and S. Wei, "Relative pose measurement algorithm of non-cooperative target based on stereo vision and ransac," International Journal ofSoft Computing And Software Engineering (JSCSE), vol. 2, 2012. 
[49] J. Kelsey, J. Byrne, M. Cosgrove, S. Seereeramand, and R. Mehra, "Visionbased relative pose estimation for autonomous rendezvous and docking," IEEE Aerospace Conference, 2006.

[50] J. Liu, Z. Liu, S. Lu, and N. Sang, "Pose estimation of non-cooperative targets without feature tracking," International Society for Optics and Photonics, 2015.

[51] F. Rosso, F. Gallo, W. Allasia, and E. Licata, "Stereo vision system for capture and removal of space debris," in Conference On Design and Architectures for Signal and Image Processing (DASIP), (Turin, Italy), pp. 201-207, 2013.

[52] D. Carlo, P. Prinetto, D. Rolfo, N. Sansonne, and P. Trotta, "A novel algorithm and hardware architecture for fast video-based shape reconstruction of space debris.," in IFIP/IEEE 21st International Conference on Very Large Scale Integration (VLSI-SoC), (Istanbul,Turkey), p. 186-191, 2013.

[53] C. Conde, F. Toledo-Moreo, R. Toledo-Moreo, J. Álvarez, J. Guerrero, and J. Vicente, "Evaluation of stereo correspondence algorithms and their implementation on fpga," in Conference on Design and Architectures for Signal and Image Processing (DASIP), p. 22-31, 2014.

[54] S. Thomas, K. Papadimitriou, and A. Dollas, "Architecture and implementation of real-time $3 \mathrm{~d}$ stereo vision on a xilinx fpga," in IFIP/IEEE 21st International Conference on Very Large Scale Integration (VLSI-SoC), (Istanbul,Turkey), p. 186-191, 2013.

[55] W. Wang, J. Yan, N. Xu, Y. Wang, and F. Hsu, "Real-time high-quality stereo vision system in fpga," in International Conference On Field-Programmable Technology, (Kyoto ,Japan)), p. 358-361, 2013.

[56] F. Yu, Z. He, B. Qiao, and X. Yu, "Stereo-vision-based relative pose estimation for the rendezvous and docking of noncooperative satellites," in Mathematical Problems in Engineering, 2014.

[57] F. Kendoul, I. Fanton, and K. Nonami, "Pose and motion estimation using dual quaternion-based extended kalman filtering," in International Society for Optics and Photonics, vol. 57, pp. 591-602, 2009.

[58] S. G. Kim, J. L. Crassidis, Y. Cheng, A. M. Fosbury, and J. L. Junkins, "Optic flow-based vision system for autonomous 3d localization and control of 
small aerial vehicles," in Journal of Guidance, Control, and Dynamics, vol. 30, pp. 133-143, 2005.

[59] M. L. Psiaki, "Autonomous orbit determination for two spacecraft from relative position measurements," in Journal of Guidance, Control and Dynamics, vol. 22, 2003.

[60] S. Chen, "Kalman filter for robot vision: A survey," IEEE Transactions on Industrial Electronics, vol. 59, pp. 4409-4420, 2012.

[61] P. T. Fung, , and M. J. Grimble, "Dynamic ship positioning using a self-tuning kalman filter," in IEEE Transactions on Automatic Control, pp. 339-350, 1983.

[62] N. Papanikolopoulos, P. Khosla, and T. Kanade, "Visual tracking of a moving target by a camera mounted on a robot - a combination of control and vision," IEEE Transactions on IEEE Trans. Robot. Autom, vol. 9, p. 14-35, 1993.

[63] M. Butala, R. Frazin, Y. Chen, and F. Kamalabadi, "Tomographic imaging of dynamic objects with the ensemble kalman filter," IEEE Transactions on IEEE Trans. Robot. Autom, vol. 18, p. 1573-1587, 2009.

[64] S. Chroust and M. Vincze, "Improvement of the prediction quality for visual servoing with a switching kalman filter," International Journal of robotics, vol. 22, p. 905-922, 2003.

[65] E. Bayro-Corrochano and Y. Zhang, "The motor extended kalman filter: A geometric approach for rigid motion estimation," International Journal of J. Math and Imag vision, vol. 13, p. 205-228, 2000.

[66] T. Cong, Y. Kim, and M. Lim, "Hybrid extended kalman filter-based localization with a highly accurate odometry model of a mobile robot," International Conf. Control and Automation Systems, p. 738-743, 2008.

[67] F. Janabi-Sharifi and M. Marey, "Kalman-filter-based method for pose estimation in visual servoing," IEEE Journal of robotics, vol. 26, p. 939-947, 2010.

[68] S. Lee and D. Kim, "Recursive unscented kalman filtering based slam using a large number of noisy observations," Journal of Control Autom, vol. 4, p. 736-747, 2006. 
[69] S. Jeon, M. Tomizuka, and T. Katou, "Kinematic kalman filter (kkf) for robot end-effector sensing," Journal of dynamic systems, measurement, and control, vol. 131, 2009.

[70] S. Jeon, M. Tomizuka, and T. Katou, "Kinematic kalman filter (kkf) for robot end-effector sensing," Jurnal of Dynamics and Systems control, vol. 131, 2009.

[71] N. Ramakoti, A. Vinay, and R. Jatoth, "Particle swarm optimization aided kalman filter for object tracking," Int. Conf. Adv. Comput.Control, Telecommun. Technol., vol. 54, p. 201-207, 2009.

[72] Z. Li, Y. Ma, and T. Ren, "Formation control of multiple mobile robots systems," Journal of robotics, vol. 5314, p. 75-82, 2008.

[73] A. Mallios, P. Ridao, D. Ribas, and E. Hernandez, "Probabilistic sonar scan matching slam for underwater environment," IEEE OCEANS, p. 1-8, 2010.

[74] M. Xujiong, H. Feng, and C. Yaowu, "Monocular simultaneous localization and mapping with a modified covariance extended kalman filter," IEEE Internatinal on intelligent and computer systems, p. 900-903, 2009.

[75] S. Holmes, G. Klein, and D. Murray, "A square root unscented kalman filter for visual monoslam," IEEE Int. Conf. Robot. Autom., p. 3710-3716, 2008.

[76] M. Chang and J. Chou, "A robust and friendly human-robot interface system based on natural human gestures," Journal of Pattern Recognit, vol. 24, p. 847-866, 2010.

[77] G. Einicke, L. White, and R. Bitmead, "The use of fake algebraic riccati equations for co-channel demodulation," in IEEE Transactions on Signal Processing, vol. 51, pp. 2288-2293, 2003.

[78] S. Bolognani, L. Tubiana, and M. Zigliotto, "Extended kalman filter tuning in sensorless pmsm drives," in IEEE Transactions on Industry Applications, vol. 39, pp. 1741-1747, 2003.

[79] K. Hoon, J. G. Lee, and C. G. Park, "Adaptive two-stage ekf for ins-gps loosely coupled system with unknown fault bias," in Journal of Global Positioning Systems, vol. 5, pp. 62-69, 2006. 
[80] H. Ahn and C. Won, "Fast alignment using rotation vector and adaptive kalman filter," in IEEE Transactions on Aerospace and Electronic Systems, vol. 42, pp. 70-83, 2006.

[81] Y. Theodor, U. Shaked, and C. Souza, "A game theory approach to robust discrete-time ho-estimation," in IEEE Transactions on Signal Processing, vol. 42, pp. 1486-1495, 1994.

[82] X.-M. Shen and L. Deng, "Game theory approach to discrete h/sub/spl infin//filter design," IEEE Transactions on Signal Processing, vol. 45, no. 4, pp. 1092-1095, 1997.

[83] W. Zhang, B. Chen, and C. Tseng, "Robust h $\infty$ filtering for nonlinear stochastic systems," in IEEE Transactions on Signal Processing, vol. 53, pp. 589-598, 2005 .

[84] D. J. Jwo and C. H. Tseng, "Fuzzy adaptive interacting multiple model unscented kalman filter for integrated navigation," Control Applications, (CCA) and Intelligent Control,(ISIC), pp. 1684-1689, 2009.

[85] G. Einicke and L. White, "Robust extended kalman filtering," in IEEE Transactions on Signal Processing, vol. 47, pp. 2596-2599, 1999.

[86] K. Reif, F. Sonnemann, and R. Unbehauen, "Nonlinear state observation using h $\infty$ filtering riccati design," in IEEE Transactions on Automatic Control, vol. 44, pp. 203-208, 1999.

[87] J. Seo, M. Yu, C. Park, and G. Lee, "An extended robust h $\infty$ filter for nonlinear constrained uncertain systems," in IEEE Transactions on Signal Processing, vol. 54, pp. 4471-4475, 2006.

[88] K. Xiong, H. Zhang, and L. Liu, "Adaptive robust extended kalman filter," in Journal of Control Theory and Applications, vol. 2, 2008.

[89] F. Busse, J. How, and J. Simpson, "Demonstration of adaptive extended kalman filter for low-earth-orbit formation estimation using cdgps," in Navigation, vol. 50, pp. 79-93, 2003.

[90] F. Jiancheng and Y. Sheng, "Study on innovation adaptive ekf for in-flight alignment of airborne pos," in EEE Transactions on lnstrumentation and Measuremen, vol. 60, pp. 1378-1388, 2011. 
[91] L. Jetto, S. Longhi, and G. Venturin, "Development and experimental validation of an adaptive extended kalman filter for the localization of mobile robots," in IEEE Transactions on Robotics and Automation, vol. 15, pp. 219-229, 1999.

[92] W. Wong and H. Lim, "Extended kalman filter based fuzzy adaptive filte," INTECH Open Access Publisher, 2009.

[93] R. Havangi, "Unscented h-infinity filtering based simultaneous localization and mapping with evolutionary resampling," Journal of the Franklin Institute, vol. 352, no. 11, pp. 4801-4825, 2015.

[94] S. Stubberud, R. Lobbia, and M. Owen, "An adaptive extended kalman filter using artificial neural networks," Proceedings of the 34th IEEE Conference on Decision and Control, vol. 2, 1995.

[95] D. J. Jwo and S. H. Wang, "Adaptive fuzzy strong tracking extended kalman filtering for gps navigation," in IEEE Journal of Sensors, vol. 7, pp. 778-789, 2007.

[96] A. Ghanbari, M. Moghanni, and B. Olyaei, "Adaptive fuzzy terminal slidingmode control of mems z-axis gyroscope with extended kalman filter observer," Journal of Systems Science and Control Engineering, pp. 183-191, 2014.

[97] Q. Song, Q. Juntong, and H. Jianda, "An adaptive ukf algorithm and its application in mobile robot control," in IEEE International Conference on Robotics and Biomimetics, pp. 1117-1122, 2006.

[98] Y. Shi, H. Chongzhao, and L. Yongqi, "Adaptive ukf for target tracking with unknown process noise statistics.," in 12th International Conference on Information Fusion, pp. 1815-1820, 2009.

[99] C. Yuping and X. Tian, "An adaptive ukf algorithm for process fault prognostics," in Second International Conference on Intelligent Computation Technology and Automation, vol. 25, pp. 487-490, 2009.

[100] J. Liu and M. Lu, "An adaptive ukf filtering algorithm for gps position estimation," in 5th International Conference on Wireless Communications, Networking and Mobile Computing, vol. 2, pp. 1-4, 2009.

[101] H. Soken and H. Chingiz, "Adaptive fading ukf with q-adaptation: application to picosatellite attitude estimation," in Journal of Aerospace Engineering, vol. 26, pp. 628-636, 2011. 
[102] D. Loebis, J. C. R. Sutton, and W. Naeem., "Adaptive tuning of a kalman filter via fuzzy logic for an intelligent auv navigation system," in Control engineering practice, vol. 12, pp. 1531-1539, 2004.

[103] K. Kobayashi, K. C. Cheok, K. Watanabe, and F. Munekata, "Localization of a wheeled mobile robot by sensor data fusion based on a fuzzy logic adapted kalman filter," in IEEE Transactions on Industrial Electronics, vol. 45, p. $510-517,1998$.

[104] L. Jetto, S. Longhi, and D. Vitali, "Fuzzy control strategy for cooperative nonholonomic motion of cybercars with passengers vibration analysis," in Control Engineering Practice, vol. 7, pp. 763-771, 1999.

[105] F. M. Raimondi and M. Melluso, "Fuzzy adaptive ekf motion control for nonholonomic and underactuated cars with parametric and non-parametric uncertainties," in IET Control Theory and Applications, pp. 1311-1321, 2007.

[106] D. Jwo, C. Yang, C. Chuang, and T. Lee, "Performance enhancement for ultra-tight gps/ins integration using a fuzzyadaptive strong tracking unscented kalman filter," in Nonlinear Dynamics, vol. 73, p. 377-395, 2013.

[107] W. Yang and S. Li, "Autonomous navigation filtering algorithm for spacecraft based on strong tracking ukf," in Systems Engineering and Electronics, vol. 33, pp. 2485-2491, 2011.

[108] Z. Ge, Y. Yang, and X. Wen, "Strong tracking ukf method and its application in fault identification," in Chinese Journal of Scientific Instrument, vol. 29, pp. 1670-1674, 2008.

[109] D. Jwo and S. Lai, "Navigation integration using the fuzzy strong tracking unscented kalman filter," in Journal of Navigation, vol. 62, pp. 503-508, 2009.

[110] Y. He, Q. Song, Y. Dong, and J. Yang, "Adaptive tracking algorithm based on modified strong tracking filter," in IEEE International Conference on Radar, pp. 1-4, 2006.

[111] S. Yazdkhasti, S. Ulrich, and J. Z. Sasiadek, "Laboratory experimentation of stereo vision-based relative navigation with unknown spinning spacecraft," in 20th IEEE/IFAC International Conference on Methods and Models in Automation and Robotics, (Miedzyzdroje, Poland), 2015. 
[112] S. Yazdkhasti, S. Ulrich, and J. Z. Sasiadek, "Computer vision for real-time relative navigation with a non-cooperative and spinning target spacecraft," in 13th Symposium on Advanced Space Technologies in Robotics and Automation, (Noordwijk, The Netherlands), 2015.

[113] S. Yazdkhasti, J. Z. Sasiadek, and S. Ulrich, "Performance enhancement for gps/ins fusion by using a fuzzy adaptive unscented kalman filter," in 21st International Conference on Methods and Models in Automation and Robotics (MMAR), pp. 1194-1199, IEEE, 2016.

[114] S. Yazdkhasti and J. Z. Sasiadek, "Adaptive h-infinity extended kalman filtering for navigation system application in presence of high uncertainties," in 17th International Conference on Informatics in Control, Automation and Robotics, ICINCO 2020, SciTePress, 2020 (Accepted).

[115] G. Bradski and A. Kaehler, "Learning opencv: Computer vision with the opencv library," in O'Reilly, (Cambridge, MA), 2008.

[116] W. Clohessy and R. Wiltshire, "Terminal guidance system for satellite rendezvous," Journal of the Aerospace Sciences, vol. 27, no. 9, pp. 653-658, 1960.

[117] P. Gurfil and K. V. Kholshevnikov, "Manifolds and metrics in the relative spacecraft motion problem," Journal of guidance, control, and dynamics, vol. 29, no. 4, pp. 1004-1010, 2006.

[118] G. G. Slabaugh, "Computing euler angles from a rotation matrix," Retrieved on August, vol. 6, no. 2000, pp. 39-63, 1999.

[119] H. S. Morton, J. L. Junkins, and J. N. Blanton, "Analytical solutions for euler parameters," Celestial mechanics, vol. 10, no. 3, pp. 287-301, 1974.

[120] M. D. Shuster, "Approximate algorithms for fast optimal attitude computation," in Guidance and Control Conference, vol. 1, pp. 88-95, 1978.

[121] J. Crassidis and F. Markley, "Attitude estimation using modified rodrigues parameters," in Nasa Conference Publication, pp. 71-86, NASA, 1996.

[122] S. Marandi and V. Modi, "A preferred coordinate system and the associated orientation representation in attitude dynamics," Acta Astronautica, vol. 15, no. 11, pp. 833-843, 1987. 
[123] M. D. Shuster et al., "A survey of attitude representations," Navigation, vol. 8, no. 9, pp. 439-517, 1993.

[124] A. Persson, "How do we understand the coriolis force," Bulletin of the American Meteorological Society, vol. 79, no. 7, pp. 1373-1386, 1998.

[125] B. E. Tweddle, "Computer vision-based localization and mapping of an unknown, uncooperative and spinning target for spacecraft proximity operations," PhD, Massachusetts Institute of Technology, Cambridge, MA, 2013.

[126] A. Papoulis and H. Saunders, "Probability, random variables and stochastic processes," 1989.

[127] M. Khalil, Bayesian inference for complex and large-scale engineering systems. PhD thesis, Carleton University, 2013.

[128] M. S. Grewal and A. P. Andrews, Kalman filtering: Theory and Practice with MATLAB. John Wiley \& Sons, 2014.

[129] B. M. Bell and F. W. Cathey, "The iterated kalman filter update as a gaussnewton method," IEEE Transactions on Automatic Control, vol. 38, no. 2, pp. 294-297, 1993.

[130] S. J. Julier and J. K. Uhlmann, "New extension of the kalman filter to nonlinear systems," in Signal processing, sensor fusion, and target recognition VI, vol. 3068, pp. 182-193, International Society for Optics and Photonics, 1997.

[131] L. A. Zadeh, "Fuzzy logic," Computer, vol. 21, no. 4, pp. 83-93, 1988.

[132] D. Dubois and H. Prade, "What are fuzzy rules and how to use them," Fuzzy sets and systems, vol. 84, no. 2, pp. 169-185, 1996.

[133] G. Klir and B. Yuan, Fuzzy sets and fuzzy logic, vol. 4. Prentice hall New Jersey, 1995.

[134] W. Van Leekwijck and E. E. Kerre, "Defuzzification: criteria and classification," Fuzzy sets and systems, vol. 108, no. 2, pp. 159-178, 1999.

[135] J. C. Piedboeuf and E. Dupuis, "Recent canadian activities in space automation and robotics-an overview," in Proceedings of the 7th International Symposium on Artificial Intelligence, Robotics, and Automation in Space: i-SAIRAS, pp. 19-23, (2003). 
[136] A. Yilmaz, O. Javed, and M. Shah, "Object tracking: A survey," Acm computing surveys (CSUR), vol. 38, no. 4, p. 13, 2006.

[137] N. Kanopoulos, N. Vasanthavada, and R. L. Baker, "Design of an image edge detection filter using the sobel operator," IEEE Journal of solid-state circuits, vol. 23, no. 2, pp. 358-367, 1988.

[138] J.-S. Chen, A. Huertas, and G. Medioni, "Fast convolution with laplacian-ofgaussian masks," IEEE Transactions on Pattern Analysis and Machine Intelligence, no. 4, pp. 584-590, 1987.

[139] W. McIlhagga, "The canny edge detector revisited," International Journal of Computer Vision, vol. 91, no. 3, pp. 251-261, 2011.

[140] K. Yang, S. Gao, C. Li, and Y. Li, "Efficient color boundary detection with color-opponent mechanisms," in Proceedings of the IEEE conference on computer vision and pattern recognition, pp. 2810-2817, 2013.

[141] D. R. Martin, C. C. Fowlkes, and J. Malik, "Learning to detect natural image boundaries using local brightness, color, and texture cues," IEEE Transactions on Pattern Analysis \& Machine Intelligence, no. 5, pp. 530-549, 2004.

[142] P. Dollar, Z. Tu, and S. Belongie, "Supervised learning of edges and object boundaries," in 2006 IEEE Computer Society Conference on Computer Vision and Pattern Recognition (CVPR'06), vol. 2, pp. 1964-1971, IEEE, 2006.

[143] X. Ren, "Multi-scale improves boundary detection in natural images," in European conference on computer vision, pp. 533-545, Springer, 2008.

[144] N. Payet and S. Todorovic, "Sledge: Sequential labeling of image edges for boundary detection," International journal of computer vision, vol. 104, no. 1, pp. 15-37, 2013.

[145] M. Maire, P. Arbelaez, C. Fowlkes, and J. Malik, "Using contours to detect and localize junctions in natural images," in 2008 IEEE Conference on Computer Vision and Pattern Recognition, pp. 1-8, IEEE, 2008.

[146] R. Xiaofeng and L. Bo, "Discriminatively trained sparse code gradients for contour detection," in Advances in neural information processing systems, pp. 584$592,2012$. 
[147] C. G. Harris, M. Stephens, et al., "A combined corner and edge detector.," in Alvey vision conference, vol. 15, pp. 10-5244, Citeseer, 1988.

[148] J. Shi and C. Tomasi, "Good features to track," tech. rep., Cornell University, 1993.

[149] P. Mainali, Q. Yang, G. Lafruit, R. Lauwereins, and L. Van Gool, "Lococo: Low complexity corner detector," in 2010 IEEE International Conference on Acoustics, Speech and Signal Processing, pp. 810-813, IEEE, 2010.

[150] S. M. Smith and J. M. Brady, "Susan - a new approach to low level image processing," International journal of computer vision, vol. 23, no. 1, pp. 45-78, 1997.

[151] K. Hariharakrishnan and D. Schonfeld, "Fast object tracking using adaptive block matching," IEEE transactions on multimedia, vol. 7, no. 5, pp. 853-859, 2005 .

[152] K. Mikolajczyk, T. Tuytelaars, C. Schmid, A. Zisserman, J. Matas, F. Schaffalitzky, T. Kadir, and L. Van Gool, "A comparison of affine region detectors," International journal of computer vision, vol. 65, no. 1-2, pp. 43-72, 2005.

[153] D. G. Lowe, "Distinctive image features from scale-invariant keypoints," International journal of computer vision, vol. 60, no. 2, pp. 91-110, 2004.

[154] H. Bay, A. Ess, T. Tuytelaars, and L. Van Gool, "Speeded-up robust features (surf)," Computer vision and image understanding, vol. 110, no. 3, pp. 346-359, 2008 .

[155] M. Agrawal, K. Konolige, and M. R. Blas, "Censure: Center surround extremas for realtime feature detection and matching," in European Conference on Computer Vision, pp. 102-115, Springer, 2008.

[156] M. Agrawal, K. Konolige, and M. R. Blas, "Censure: Center surround extremas for realtime feature detection and matching," in European Conference on Computer Vision, pp. 102-115, Springer, 2008.

[157] G. Olague and L. Trujillo, "Interest point detection through multiobjective genetic programming," Applied Soft Computing, vol. 12, no. 8, pp. 2566-2582, 2012 . 
[158] P. F. Alcantarilla, A. Bartoli, and A. J. Davison, "Kaze features," in European Conference on Computer Vision, pp. 214-227, Springer, 2012.

[159] E. Rublee, V. Rabaud, K. Konolige, and G. Bradski, "Orb: An efficient alternative to sift or surf," 2011.

[160] S. Leutenegger, M. Chli, and R. Siegwart, "Brisk: Binary robust invariant scalable keypoints," in 2011 IEEE international conference on computer vision (ICCV), pp. 2548-2555, IEEE, 2011.

[161] A. Alahi, R. Ortiz, and P. Vandergheynst, "Freak: Fast retina keypoint," in 2012 IEEE Conference on Computer Vision and Pattern Recognition, pp. 510517, IEEE, 2012.

[162] P. Forssén and D. G. Lowe, "Shape descriptors for maximally stable extremal regions," in 2007 IEEE 11th International Conference on Computer Vision, pp. 1-8, IEEE, 2007.

[163] T. Tuytelaars and L. Van Gool, "Matching widely separated views based on affine invariant regions," International journal of computer vision, vol. 59, no. 1, pp. 61-85, 2004.

[164] T. Kadir, A. Zisserman, and M. Brady, "An affine invariant salient region detector," in European conference on computer vision, pp. 228-241, Springer, 2004.

[165] X. Hou and L. Zhang, "Saliency detection: A spectral residual approach," in 2007 IEEE Conference on computer vision and pattern recognition, pp. 1-8, IEEE, 2007.

[166] S. Gu, Y. Zheng, and C. Tomasi, "Critical nets and beta-stable features for image matching," in European Conference on computer vision, pp. 663-676, Springer, 2010.

[167] Y. Avrithis and K. Rapantzikos, "The medial feature detector: Stable regions from image boundaries," in 2011 International Conference on Computer Vision, pp. 1724-1731, IEEE, 2011.

[168] P.-E. Forssén and D. G. Lowe, "Shape descriptors for maximally stable extremal regions," in 2007 IEEE 11th International Conference on Computer Vision, pp. 1-8, IEEE, 2007. 
[169] D. M. Gavrila and L. S. Davis, "3-d model-based tracking of humans in action: a multi-view approach," in Proceedings CVPR IEEE Computer Society Conference on Computer Vision and Pattern Recognition, pp. 73-80, IEEE, (1996).

[170] V. Lepetit, P. Fua, et al., "Monocular model-based 3d tracking of rigid objects: A survey," Foundations and Trends@ in Computer Graphics and Vision, vol. 1, no. 1, pp. 1-89, 2005.

[171] I. Oikonomidis, N. Kyriazis, and A. A. Argyros, "Efficient model-based 3d tracking of hand articulations using kinect.," in BmVC, vol. 1, p. 3, 2011.

[172] E. L. Andrade, J. C. Woods, E. Khan, and M. Ghanbari, "Region-based analysis and retrieval for tracking of semantic objects and provision of augmented information in interactive sport scenes," IEEE Transactions on Multimedia, vol. 7, no. 6, pp. 1084-1096, 2005.

[173] S. T. Birchfield and S. Rangarajan, "Spatiograms versus histograms for regionbased tracking," in Computer Vision and Pattern Recognition, 2005. CVPR 2005. IEEE Computer Society Conference on, vol. 2, pp. 1158-1163, IEEE, 2005 .

[174] S. T. Birchfield and S. Rangarajan, "Spatial histograms for region-based tracking," ETRI journal, vol. 29, no. 5, pp. 697-699, 2007.

[175] K. Yang, Z. Cai, and L. Zhao, "Algorithm research on moving object detection of surveillance video sequence," Optics and Photonics Journal, vol. 3, no. 02, p. 308,2013

[176] R. Cucchiara, M. Piccardi, and P. Mello, "Image analysis and rule-based reasoning for a traffic monitoring system," IEEE Transactions on Intelligent Transportation Systems, vol. 1, no. 2, pp. 119-130, 2000.

[177] B. N. Subudhi, P. K. Nanda, and A. Ghosh, "A change information based fast algorithm for video object detection and tracking," IEEE Transactions on Circuits and Systems for Video Technology, vol. 21, no. 7, pp. 993-1004, 2011.

[178] N. Saunier and T. Sayed, "A feature-based tracking algorithm for vehicles in intersections," in Computer and Robot Vision, 2006. The 3rd Canadian Conference on, pp. 59-59, IEEE, (2006). 
[179] Z. Chen and S. T. Birchfield, "Person following with a mobile robot using binocular feature-based tracking," in Intelligent Robots and Systems, 200\%. IROS 200\%. IEEE/RSJ International Conference on, pp. 815-820, IEEE, 2007.

[180] A. Prioletti, A. Møgelmose, P. Grisleri, M. M. Trivedi, A. Broggi, and T. B. Moeslund, "Part-based pedestrian detection and feature-based tracking for driver assistance: real-time, robust algorithms, and evaluation," IEEE Transactions on Intelligent Transportation Systems, vol. 14, no. 3, pp. 1346-1359, 2013.

[181] G. Devetag, S. Di Guida, and L. Polonio, "An eye-tracking study of featurebased choice in one-shot games," Experimental Economics, vol. 19, no. 1, pp. 177-201, 2016.

[182] D. Koller, K. Daniilidis, and H.-H. Nagel, "Model-based object tracking in monocular image sequences of road traffic scenes," International Journal of Computer 11263on, vol. 10, no. 3, pp. 257-281, 1993.

[183] A. Cavallaro, O. Steiger, and T. Ebrahimi, "Tracking video objects in cluttered background," IEEE Transactions on Circuits and Systems for Video Technology, vol. 15, no. 4, pp. 575-584, 2005.

[184] G. D. Hager and P. N. Belhumeur, "Efficient region tracking with parametric models of geometry and illumination," IEEE transactions on pattern analysis and machine intelligence, vol. 20, no. 10, pp. 1025-1039, 1998.

[185] B. Deori and D. M. Thounaojam, "A survey on moving object tracking in video," International Journal on Information Theory (IJIT), vol. 3, no. 3, pp. 31-46, 2014.

[186] R. E. Kalman, "A new approach to linear filtering and prediction problems," Journal of basic Engineering, vol. 82, no. 1, pp. 35-45, 1960.

[187] Y. Bar-Shalom and X.-R. Li, Multitarget-multisensor tracking: principles and techniques, vol. 19. YBs London, UK:, (1995).

[188] J. Zhu, N. Zheng, Z. Yuan, Q. Zhang, X. Zhang, and Y. He, "A slam algorithm based on the central difference kalman filter," in Intelligent Vehicles Symposium, 2009 IEEE, pp. 123-128, IEEE, 2009. 
[189] A. Adam, E. Rivlin, and I. Shimshoni, "Robust fragments-based tracking using the integral histogram," in Computer Society Conference on Computer vision and pattern recognition, vol. 1, pp. 798-805, IEEE, (2006).

[190] K. Zhang, L. Zhang, and M.-H. Yang, "Real-time object tracking via online discriminative feature selection," IEEE Transactions on Image Processing, vol. 22, no. 12 , pp. 4664-4677, 2013.

[191] K. Okuma, A. Taleghani, N. De Freitas, J. J. Little, and D. G. Lowe, "A boosted particle filter: Multitarget detection and tracking," in European conference on computer vision, pp. 28-39, Springer, 2004.

[192] M. S. Arulampalam, S. Maskell, N. Gordon, and T. Clapp, "A tutorial on particle filters for online nonlinear/non-gaussian bayesian tracking," IEEE Transactions on signal processing, vol. 50, no. 2, pp. 174-188, 2002.

[193] S. K. Weng, C. M. Kuo, and S.-K. Tu, "Video object tracking using adaptive kalman filter," Journal of Visual Communication and Image Representation, vol. 17, no. 6, pp. 1190-1208, 2006.

[194] M. E. Hough, "Improved performance of recursive tracking filters using batch initialization and process noise adaptation," Journal of guidance, control, and dynamics, vol. 22, no. 5, pp. 675-681, 1999.

[195] S. Ruel, T. Luu, and A. Berube, "Space shuttle testing of the tridar 3d rendezvous and docking sensor," Journal of Field robotics, vol. 29, no. 4, pp. 535$553,2012$.

[196] L. Ljung, "Asymptotic behavior of the extended kalman filter as a parameter estimator for linear systems," IEEE Transactions on Automatic Control, vol. 24, no. 1, pp. 36-50, 1979.

[197] S. J. Julier and J. K. Uhlmann, "Unscented filtering and nonlinear estimation," Proceedings of the IEEE, vol. 92, no. 3, pp. 401-422, 2004.

[198] J. Z. Sasiadek, "Sensor fusion," Annual Reviews in Control, vol. 26, no. 2, pp. 203-228, 2002.

[199] M. Kayton and W. R. Fried, Avionics navigation systems. John Wiley \& Sons, 1997. 
[200] S. Gauglitz, T. Höllerer, and M. Turk, "Evaluation of interest point detectors and feature descriptors for visual tracking," International journal of computer vision, vol. 94, no. 3, pp. 335-360, 2011.

[201] E. A. Wan and R. Van Der Merwe, "The unscented kalman filter for nonlinear estimation," in Adaptive Systems for Signal Processing, Communications, and Control Symposium 2000., pp. 153-158, IEEE, (2000).

[202] N. Inaba and M. Oda, "Autonomous satellite capture by a space robot: world first on-orbit experiment on a japanese robot satellite ets-vii," in IEEE International Conference on Robotics and Automation, vol. 2, pp. 1169-1174, 2000.

[203] R. T. Howard, A. F. Heaton, R. M. Pinson, and C. K. Carrington, "Orbital express advanced video guidance sensor," in IEEE Aerospace Conference, pp. 1$10,2008$.

[204] ESA, "Europe's automated ship docks to the iss," 2008.

[205] D. Fourie, B. E. Tweddle, S. Ulrich, and A. Saenz-Otero, "Flight results of vision-based navigation for autonomous spacecraft inspection of unknown objects," Journal of Spacecraft and Rockets, vol. 51, no. 6, pp. 2016-2026, 2014.

[206] X. Zhang, Z. Zhang, Y. Li, X. Zhu, Q. Yu, and J. Ou, "Robust camera pose estimation from unknown or known line correspondences," Applied optics, vol. 51, no. 7, pp. 936-948, 2012.

[207] F. Aghili, M. Kuryllo, G. Okouneva, and C. English, "Robust vision-based pose estimation of moving objects for automated rendezvous \& docking," in IEEE International Conference on Mechatronics and Automation (ICMA), pp. 305$311,2010$.

[208] X. Du, B. Liang, W. Xu, and Y. Qiu, "Pose measurement of large noncooperative satellite based on collaborative cameras," Acta Astronautica, vol. 68, no. 11, pp. 2047-2065, 2011.

[209] S. Segal, A. Carmi, and P. Gurfil, "Stereovision-based estimation of relative dynamics between noncooperative satellites: theory and experiments," IEEE Transactions on Control Systems Technology, vol. 22, no. 2, pp. 568-584, 2014.

[210] G. Hu, S. Gao, and Y. Zhong, "A derivative ukf for tightly coupled ins/gps integrated navigation," ISA transactions, vol. 56, pp. 135-144, 2015. 
[211] Y. Huang, Y. Zhang, B. Xu, Z. Wu, and J. A. Chambers, "A new adaptive extended kalman filter for cooperative localization," IEEE Transactions on Aerospace and Electronic Systems, vol. 54, no. 1, pp. 353-368, 2017.

[212] H. Wang, Z. Deng, B. Feng, H. Ma, and Y. Xia, "An adaptive kalman filter estimating process noise covariance," Neurocomputing, vol. 223, pp. 12-17, 2017.

[213] P. J. Escamilla-Ambrosio and N. Mort, "Multi-sensor data fusion architecture based on adaptive kalman filters and fuzzy logic performance assessment," in Proceedings of the Fifth International Conference on Information Fusion. FUSION 2002.(IEEE Cat. No. 02EX5997), vol. 2, pp. 1542-1549, IEEE, 2002.

[214] X. Gong, J. Suh, and C. Lin, "A novel method for identifying inertial parameters of electric vehicles based on the dual h infinity filter," Vehicle System Dynamics, vol. 58, no. 1, pp. 28-48, 2020.

[215] D. Simon, Optimal state estimation: Kalman, H infinity, and nonlinear approaches. John Wiley \& Sons, 2006.

[216] M. J. Grimble and A. El Sayed, "Solution of the h/sub infinity/optimal linear filtering problem for discrete-time systems," IEEE Transactions on Acoustics, Speech, and Signal Processing, vol. 38, no. 7, pp. 1092-1104, 1990.

[217] B. Hassibi, A. H. Sayed, and T. Kailath, "Linear estimation in krein spaces. ii. applications," IEEE Transactions on Automatic Control, vol. 41, no. 1, pp. 3449, 1996.

[218] J. Zhao, "Dynamic state estimation with model uncertainties using hinfty extended kalman filter," IEEE Transactions on Power Systems, vol. 33, no. 1, pp. 1099-1100, 2017.

[219] F. Yu, Q. Sun, C. Lv, Y. Ben, and Y. Fu, "A slam algorithm based on adaptive cubature kalman filter," Mathematical Problems in Engineering, 2014.

[220] Y. Yang and W. Gao, "An optimal adaptive kalman filter," Journal of Geodesy, vol. 80, no. 4, pp. 177-183, 2006.

[221] F. Aghili and K. Parsa, "An adaptive vision system for guidance of a robotic manipulator to capture a tumbling satellite with unknown dynamics," in IEEE Intelligent Robots and Systems IROS, (Oregon), p. 3064-3071, 2008. 
[222] X. Tong, Z. Li, G. Han, N. Liu, Y. Su, J. Ning, and F. Yang, "Adaptive ekf based on hmm recognizer for attitude estimation using mems marg sensors," IEEE Sensors Journal, vol. 18, no. 8, pp. 3299-3310, 2017.

[223] X. Shao, B. He, J. Guo, and T. Yan, "The application of auv navigation based on adaptive extended kalman filter," in Oceans 2016-Shanghai, pp. 1-4, IEEE, 2016.

[224] R. Kottath, P. Narkhede, V. Kumar, V. Karar, and S. Poddar, "Multiple model adaptive complementary filter for attitude estimation," Aerospace Science and Technology, vol. 69, pp. 574-581, 2017.

[225] L. Jetto, S. Longhi, and G. Venturini, "Development and experimental validation of an adaptive extended kalman filter for the localization of mobile robots," IEEE Transactions on Robotics and Automation, vol. 15, no. 2, pp. 219-229, 1999.

[226] K. H. Kim, J. G. Lee, and C. G. Park, "Adaptive two-stage extended kalman filter for a fault-tolerant ins-gps loosely coupled system," IEEE Transactions on Aerospace and Electronic Systems, vol. 45, no. 1, pp. 125-137, 2009.

[227] S. C. Stubberud, R. N. Lobbia, and M. Owen, "An adaptive extended kalman filter using artificial neural networks," in Proceedings of 1995 34th IEEE Conference on Decision and Control, vol. 2, pp. 1852-1856, IEEE, 1995.

[228] Y. Shi, C. Han, and Y. Liang, "Adaptive ukf for target tracking with unknown process noise statistics," in 2009 12th International Conference on Information Fusion, pp. 1815-1820, IEEE, 2009.

[229] H. E. Soken and C. Hajiyev, "Adaptive fading ukf with q-adaptation: application to picosatellite attitude estimation," Journal of Aerospace Engineering, vol. 26, no. 3, pp. 628-636, 2011.

[230] W. Wang, Z.-y. Liu, and R.-r. Xie, "Quadratic extended kalman filter approach for gps/ins integration," Aerospace science and technology, vol. 10, no. 8, pp. 709-713, 2006.

[231] M. Tehrani, N. Nariman-zadeh, and M. Masoumnezhad, "Adaptive fuzzy hybrid unscented/h-infinity filter for state estimation of nonlinear dynamics problems," Transactions of the Institute of Measurement and Control, vol. 41, no. 6, pp. 1676-1685, 2019. 
[232] J. Zhao and L. Mili, "A theoretical framework of robust h-infinity unscented kalman filter and its application to power system dynamic state estimation," IEEE Transactions on Signal Processing, vol. 67, no. 10, pp. 2734-2746, 2019.

[233] F. L. Lewis, L. Xie, and D. Popa, Optimal and robust estimation: with an introduction to stochastic control theory. CRC press, 2017.

[234] J. Sasiadek and P. Hartana, "Sensor fusion for navigation of an autonomous unmanned aerial vehicle," in Robotics and Automation, 2004. Proceedings. ICRA'04. 2004 IEEE International Conference on, vol. 4, pp. 4029-4034, IEEE, 2004.

[235] B. Hassibi, A. H. Sayed, and T. Kailath, Indefinite-Quadratic estimation and control: a unified approach to $H_{2}$ and $H \infty$ theories. SIAM, 1999.

[236] Y. Liu, X. Fan, C. Lv, J. Wu, L. Li, and D. Ding, "An innovative information fusion method with adaptive kalman filter for integrated ins/gps navigation of autonomous vehicles," Mechanical Systems and Signal Processing, vol. 100, pp. 605-616, 2018.

[237] Y. Yang, X. Ren, and Y. Xu, "Main progress of adaptively robust filter with applications in navigation," J. Navig. Position, vol. 1, pp. 9-15, 2013.

[238] H. E. Soken, C. Hacizade, and S.-i. Sakai, "Simultaneous adaptation of the process and measurement noise covariances for the ukf applied to nanosatellite attitude estimation," IFAC Proceedings Volumes, vol. 47, no. 3, pp. 5921-5926, 2014.

[239] P. Lu, L. Zhao, and Z. Chen, "Improved sage-husa adaptive filtering and its application," Journal of System Simulation, vol. 15, pp. 3503-3505, 2007.

[240] D. Gaylor and E. G. Lightsey, "Gps/ins kalman filter design for spacecraft operating in the proximity of international space station," in AIAA Guidance, Navigation, and Control Conference and Exhibit, p. 5445, 2003.

[241] W. Van Etten, Introduction to random signals and noise. Wiley Online Library, 2005.

[242] D. Simon, Optimal state estimation: Kalman, H infinity, and nonlinear approaches. John Wiley \& Sons, 2006. 


\section{Appendix A}

\section{A.1 GPS Satellite Geometry}

The true range of Four pseudorange equations for GPS measurements can be defined as:

$$
\begin{aligned}
& h_{1}=\sqrt{\left(X_{1}-x\right)^{2}+\left(Y_{1}-y\right)^{2}+\left(Z_{1}-z\right)^{2}}+C \Delta t_{1} \\
& h_{2}=\sqrt{\left(X_{2}-x\right)^{2}+\left(Y_{2}-y\right)^{2}+\left(Z_{2}-z\right)^{2}}+C \Delta t_{2} \\
& h_{3}=\sqrt{\left(X_{3}-x\right)^{2}+\left(Y_{3}-y\right)^{2}+\left(Z_{3}-z\right)^{2}}+C \Delta t_{3} \\
& h_{4}=\sqrt{\left(X_{4}-x\right)^{2}+\left(Y_{4}-y\right)^{2}+\left(Z_{4}-z\right)^{2}}+C \Delta t_{4}
\end{aligned}
$$

where, $\left(X_{1}, Y_{1}, Z_{1}\right),\left(X_{2}, Y_{2}, Z_{2}\right),\left(X_{3}, Y_{3}, Z_{3}\right),\left(X_{4}, Y_{4}, Z_{4}\right)$ are the positions of the four GPS satellites respectively, and $(x, y, z)$ are the position of the vehicle. The general form of total measured pseudorange can be defined as:

Total measured pseudorange $=$ True range + Range bias + White measurement noise 
Although the satellite positions are reported in WGS-84 coordinates, it is sometimes useful to deal with a locally level frame of reference, where the $x^{\prime}-y^{\prime}$ plane is tangential to the surface of the earth ellipsoid. As depicted in Figure. A.1, we shall define such a locally level reference frame by having the $x^{\prime}$-axis pointing east, the $y^{\prime}$-axis north, and the $z^{\prime}$-axis pointed up locally. It suffices here to say that the coordinate transformations to convert between the WGS-84 coordinates and any other derived reference frame, including the locally level one given here, are usually quite straightforward [7].

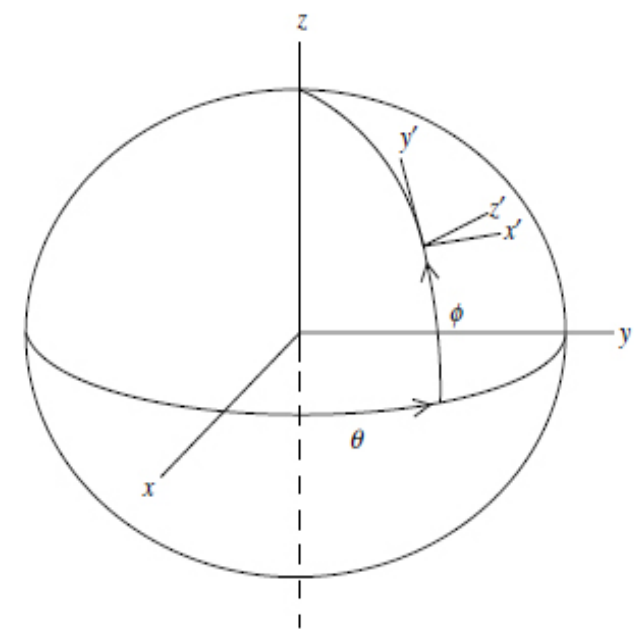

Figure. A.1. The WGS-84 coordinate reference frame $(x, y, z)$ used by GPS and a locally level coordinate reference frame $\left(x^{\prime}, y^{\prime}, z^{\prime}\right)[7]$.

The GPS satellite assumed to be in circular orbits. The Satellite positions in Earth-Center Earth-fixed coordinate can be obtained by:

$$
\begin{gathered}
X_{j}=R\left[\cos \theta_{j} \cos \Omega_{j}-\sin \theta_{j} \sin \Omega_{j} \cos 55^{\circ}\right] \\
Y_{j}=R\left[\cos \theta_{j} \sin \Omega_{j}+\sin \theta_{j} \sin \Omega_{j} \cos 55^{\circ}\right] \\
Z_{j}=R\left[\sin \theta_{j} \sin 55^{\circ}\right]
\end{gathered}
$$


where

$$
\begin{gathered}
\theta_{j}=\theta_{0}+T \frac{360}{43082} \operatorname{deg} \quad j=1, \ldots, 4 \\
\Omega_{j}=\Omega_{0}-T \frac{360}{86164} \operatorname{deg} \\
R=26560000 m
\end{gathered}
$$

In the simulation to avoid the complications of transformation a locally level coordinate frame was used. The locally level coordinate frame consider coincide with the observers (receiver) local reference. The satellites positions in the locally level coordinate frame can be written as function of time as follow [7]:

$$
\begin{gathered}
X_{j}(t)=R\left[\cos \theta_{j}(t) \cos \Omega_{j}(t)+\sin \theta_{j}(t) \sin \Omega_{j}(t) \cos 55^{\circ}\right] \\
Y_{j}(t)=R\left[\sin \theta_{j}(t) \sin 55^{\circ}\right] \\
Z_{j}(t)=R\left[\cos \theta_{j}(t) \cos \Omega_{j}(t)-\sin \theta_{j}(t) \sin \Omega_{j}(t) \cos 55^{\circ}\right]-r
\end{gathered}
$$

where $r$ is the earth radius $(\mathrm{r}=-6380 \mathrm{~km})$.

Table A.1: Satellite parameters [3].

\begin{tabular}{crr}
\hline \hline GPS Satellite & $\Omega_{0}^{\circ}$ & $\theta_{0}^{\circ}$ \\
\hline Satellite 1 & 326 & 68 \\
Satellite 2 & 26 & 340 \\
Satellite 3 & 146 & 198 \\
Satellite 4 & 86 & 271 \\
\hline \hline
\end{tabular}




\section{A.1.1 Error sources of GPS}

The major sources of GPS's errors are summarized in Table A.2 [3].

Table A.2: Error sources of GPS

\begin{tabular}{crr}
\hline \hline Error Component & Standard Deviation & GPS Control Segment \\
\hline Satellite Position & $3.3 \mathrm{~m}$ & GPS Control Segment \\
Lono Reflection with Correction & $1.5 \mathrm{~m}$ & Solar Flare Activity \\
Tropo Reflection with Correction & $2.2 \mathrm{~m}$ & Local atmospheric Condition \\
Multipath & $1.5 \mathrm{~m}$ & Low Elevation $\left.\left(<15^{\circ}\right)\right)$ \\
Selective Availability & $0.3 \mathrm{~m}$ & GPS Control Segment \\
\hline \hline
\end{tabular}

